Florida International University FIU Digital Commons

$1-24-2018$

\title{
Plasmonic Nanoplatforms for Biochemical Sensing and Medical Applications
}

Arash Ahmadivand

Florida International University, aahma011@fiu.edu

DOI: 10.25148 /etd.FIDC004068

Follow this and additional works at: https://digitalcommons.fiu.edu/etd

Part of the Biomedical Commons, Electrical and Electronics Commons, Electromagnetics and Photonics Commons, and the Nanotechnology Fabrication Commons

\section{Recommended Citation}

Ahmadivand, Arash, "Plasmonic Nanoplatforms for Biochemical Sensing and Medical Applications" (2018). FIU Electronic Theses and Dissertations. 3576.

https://digitalcommons.fiu.edu/etd/3576

This work is brought to you for free and open access by the University Graduate School at FIU Digital Commons. It has been accepted for inclusion in FIU Electronic Theses and Dissertations by an authorized administrator of FIU Digital Commons. For more information, please contact dcc@fiu.edu. 


\section{FLORIDA INTERNATIONAL UNIVERSITY}

Miami, Florida

\section{PLASMONIC NANOPLATFORMS FOR BIOCHEMICAL SENSING AND MEDICAL APPLICATIONS}

A dissertation submitted in partial fulfillment of

the requirements for the degree of

DOCTOR OF PHILOSOPHY

in

ELECTRICAL ENGINEERING

by

Arash Ahmadivand 
To: John L. Volakis

College of Engineering and Computing

This dissertation, written by Arash Ahmadivand, and entitled Plasmonic Nanoplatforms for Biochemical Sensing and Medical Applications, having been approved in respect to style and intellectual content, is referred to you for judgment.

We have read this dissertation and recommend that it be approved.

Shekhar Bhansali

Sakhrat Khizroev

Ajeet Kaushik

Nezih Pala, Major Professor

Date of Defense: January 24, 2018

The thesis of Arash Ahmadivand is approved.

John L. Volakis

College of Engineering and Computing

Andrés G. Gil

Vice President for Research and Economic Development and Dean of the University Graduate School

Florida International University, 2018 
(C) Copyright 2018 by Arash Ahmadivand

All rights reserved. 


\section{DEDICATION}

I dedicate this dissertation to my loving parents and brother. Without their love, support, and patience, it would have been impossible to complete this research. 


\section{ACKNOWLEDGMENTS}

First and foremost, I would like to express gratitude to my advisor and mentor, Dr. Nezih Pala, for giving me the opportunity to work on an exciting project for my doctoral dissertation. I am also thankful for his encouragement and support in developing my research skills and personality through the many opportunities provided during my time at Florida International University.

I would like to thank the members of my dissertation committee Dr. Ajeet Kaushik, Dr. Sakhrat Khizroev, and Dr. Shekhar Bhansali, for agreeing to be my dissertation committee, and for their valuable inputs towards my dissertation research. I would also like to thank Dr. Ajeet Kaushik for his support and constant thought provoking discussions. I thank the Department of Electrical and Computer Engineering, for the logistical support. I would like to thank the Graduate \& Professional Student Committee (GPSC) for the support received through conference travel grants to present my research at many national conferences.

I take this opportunity to also thank all the members of the INSYST Lab and other graduate students that worked along with me at Electrical and Computer Engineering and Advanced Materials Engineering Research Lab. Many thanks to my friend who has helped me and stood by me through the hardest times of this journey, Burak Gerislioglu, and also thanks to AMERI managers and staffs for their supports, especially Patrick Roman and Dr. Alexander Franco. I would also like to thank Prof. Andrey E. Miroshnichenko, Prof. Yogendra Kumar Mishra and Prof. Kambiz Abedi for their kind supports and helps during my $\mathrm{PhD}$ studies. Moreover, I would like to acknowledge the 
funding sources that made this work possible: Graduate teaching assistantship from the Department of Electrical and Computer Engineering at Florida International University.

Finally, I could not have come this far without the unconditional love, support, patience and blessings from my family. Many thanks to my father, Abdollah Ahmadivand, mother, Fahimeh Aghaalipour and brother, Aidin Ahmadivand for their support and unconditional love. 


\section{ABSTRACT OF THE DISSERTATION}

\section{PLASMONIC NANOPLATFORMS FOR BIOCHEMICAL SENSING AND MEDICAL}

APPLICATIONS

by

Arash Ahmadivand

Florida International University, 2018

Miami, Florida

Professor Nezih Pala, Major Professor

Plasmonics, the science of the excitation of surface plasmon polaritons (SPP) at the metal-dielectric interface under intense beam radiation, has been studied for its immense potential for developing numerous nanophotonic devices, optical circuits and lab-on-achip devices. The key feature, which makes the plasmonic structures promising is the ability to support strong resonances with different behaviors and tunable localized hotspots, excitable in a wide spectral range. Therefore, the fundamental understanding of light-matter interactions at subwavelength nanostructures and use of this understanding to tailor plasmonic nanostructures with the ability to sustain high-quality tunable resonant modes are essential toward the realization of highly functional devices with a wide range of applications from sensing to switching.

We investigated the excitation of various plasmonic resonance modes (i.e. Fano resonances, and toroidal moments) using both optical and terahertz (THz) plasmonic 
metamolecules. By designing and fabricating various nanostructures, we successfully predicted, demonstrated and analyzed the excitation of plasmonic resonances, numerically and experimentally. A simple comparison between the sensitivity and lineshape quality of various optically driven resonances reveals that nonradiative toroidal moments are exotic plasmonic modes with strong sensitivity to environmental perturbations. Employing toroidal plasmonic metasurfaces, we demonstrated ultrafast plasmonic switches and highly sensitive sensors. Focusing on the biomedical applications of toroidal moments, we developed plasmonic metamaterials for fast and cost-effective infection diagnosis using the $\mathrm{THz}$ range of the spectrum. We used the exotic behavior of toroidal moments for the identification of Zika-virus (ZIKV) envelope proteins as the infectious nano-agents through two protocols: 1) direct biding of targeted biomarkers to the plasmonic metasurfaces, and 2) attaching gold nanoparticles to the plasmonic metasurfaces and binding the proteins to the particles to enhance the sensitivity. This led to developing ultrasensitive $\mathrm{THz}$ plasmonic metasensors for detection of nanoscale and low-molecular-weight biomarkers at the picomolar range of concentration.

In summary, by using high-quality and pronounced toroidal moments as sensitive resonances, we have successfully designed, fabricated and characterized novel plasmonic toroidal metamaterials for the detection of infectious biomarkers using different methods. The proposed approach allowed us to compare and analyze the binding properties, sensitivity, repeatability, and limit of detection of the metasensing devices. 


\section{TABLE OF CONTENTS}

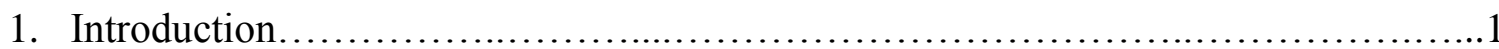

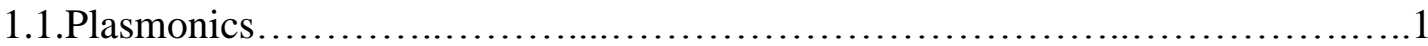

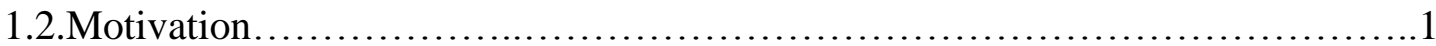

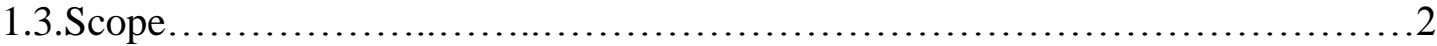

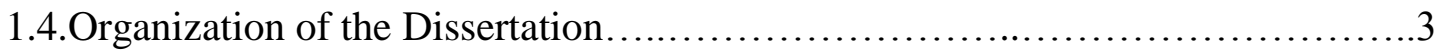

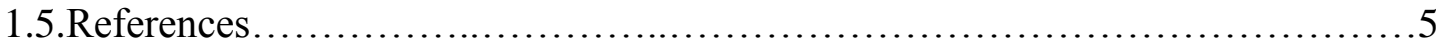

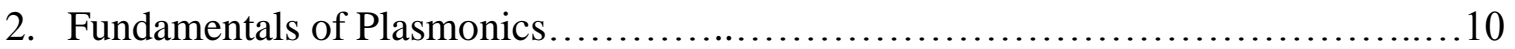

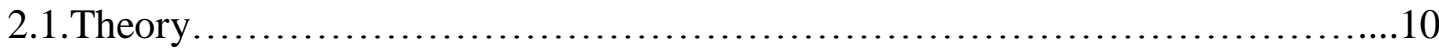

2.2.Numerical Methods..........................................................

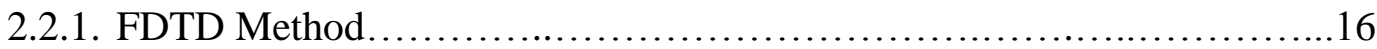

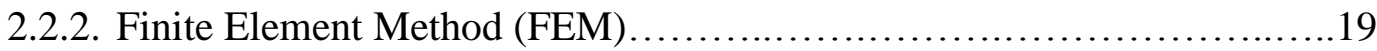

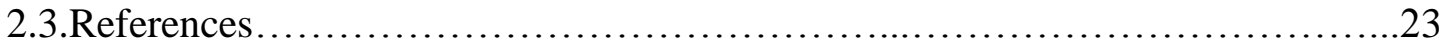

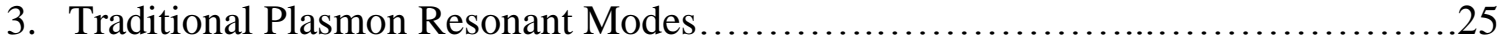

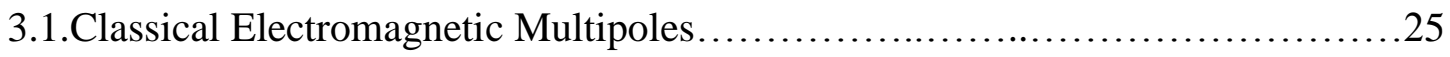

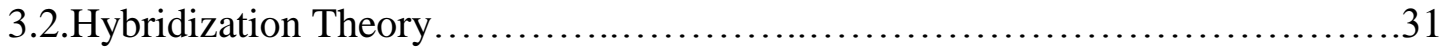

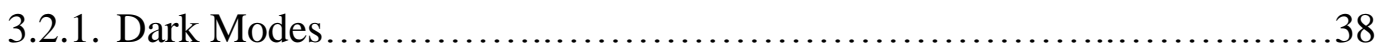

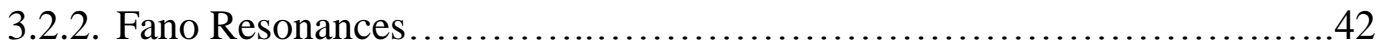

3.2.2.1. Fano-Resonant Asymmetric Nanoring Clusters...................44

3.2.2.2. Fano-Resonant Al Clusters for UV Band..........................48

3.2.2.3. Fano-Resonant Biochemical Sensors.............................49

3.2.2.4. Fano-Resonant Plasmonic UV Photodetectors.....................59

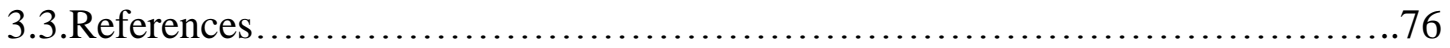

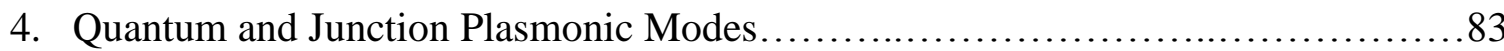

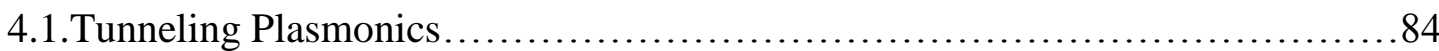

4.2.Plasmonic Switching Based on Direct Charge Transport.........................89

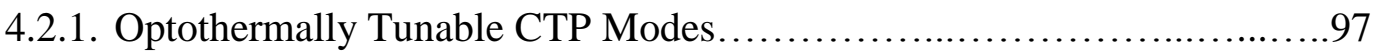

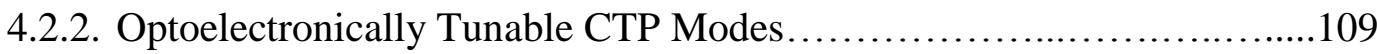

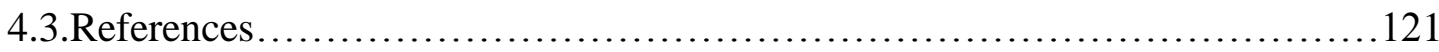

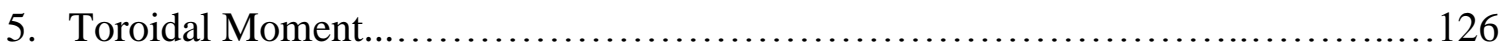

5.1.Dynamic Toroidal Dipoles and Static Multipoles.............................127 
5.1.1. Theory of Toroidal Dipolar Excitation............................... 128

5.1.2. The Toroidal Multipoles Excitation Power.................................137

5.1.3. Numerical and Experimental Observation of Toroidal Moments........139

5.2.Tunable Toroidal Resonances............................................. 146

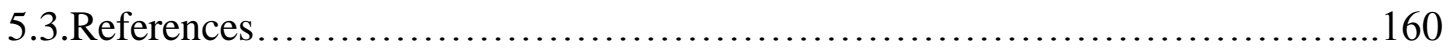

6. Plasmonics for Photothermal Heat Generation.................................. 166

6.1.Photothermal Heat Generation............................................ 166

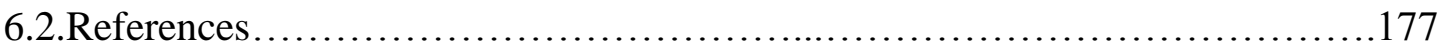

7. Terahertz Toroidal Plasmonic Metamaterial for Biomarker Protein Detection......182

7.1. Targeted Infectious (ZIKA-Virus) .................................... 183

7.2. Tailoring Bimetallic Multipixel Toroidal Unit Cells............................186

7.2.1. The Spectral Properties...................................... 187

7.2.2. The Detection Performance..........................................193

7.2.3. Conclusions ...................................................200

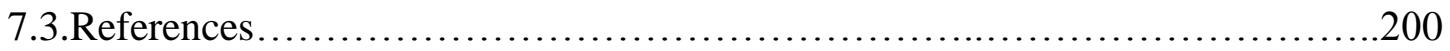

8. Nanoparticles-Enhanced Terahertz Toroidal Metasurface for Biomarker Proteins Detection .......................................................204

8.1.The Influence of Gold Nanoparticles on Toroidal Momentum..................204

8.1.1. The Spectral Properties...........................................204

8.1.2. The Detection Performance....................................210

8.1.3. Conclusions.................................................216

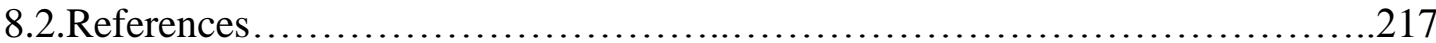

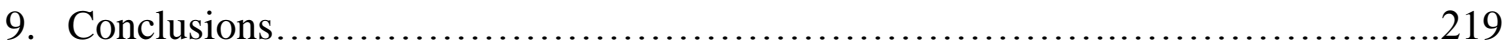

9.1.Key Contributions of the Dissertation.................................. 221

9.2.Recommendations for the Future Work...................................222

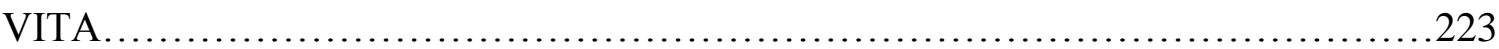




\section{LIST OF FIGURES}

FIGURE

2.1 Surface plasmon resonance excitation. a) Schematic and b) cross-sectional representations for the excitation and oscillation of plasmons at the metaldielectric interface

2.2 Scheme of the dispersion relation diagram of nonradiative plasmons. a) Dispersion relation as a function of real part and b) imaginary part of wave vector.

2.3 SPR excitation setups. a) Otto configuration, b) Kretschmann configuration .16

3.1 A representation of plasmonic nanomatryoshka.....

3.2 An energy level diagram, for the hybridization model in metallic nanoshells resulting interaction between the sphere and cavity plasmons

3.3 Schematic art picture of a dimer antenna in hybridized condition

3.4 Energy level diagram for plasmon resonances coupling in a) classical nearfield and b) hybridized regimes for a dimer nanostructure.

3.5 Plasmonic response of an Al nanodimer. The extinction spectra and the Efield map are calculated numerically. The resonant multipolar peaks are denoted by $\mathbf{P}$

3.6 Schematic of interference between the superradiant and subradiant modes in a unit cell.....

3.7 a) Schematic and b) top-view image of an eight-member octamer cluster composed of gold nanorings on a multilayer substrate.

3.8 The plasmonic responses for the octamer cluster composed of Au nanoshells. a) The scattering spectra for the octamer assembly, while the inner radius of central particles is variant. b) The spectral responses for the octamer cluster composed of $\mathrm{Au}$ shells under transverse electric polarization mode, while the outer radius of central particles is variant. c) The two-dimensional snapshot of plasmon resonance excitation inside the nanoshells and its coupling between proximal particles 
3.9 Plasmonic Response of a single-stone ring-shapes structure. a) Scattering cross-section for the formation of Fano dip. b), and c) Charge distribution and Efield maps at the Fano dip position, respectively

3.10 Plasmonic response of an Al heptamer. a) Schematic of the Al heptamer with a coverage of oxide layer around nanodisks. b) Scattering and absorption crosssection for the formation of Fano dip. c) E-field maps at the Fano dip position

3.11 Plasmonic response of an Al decamer. a) and b) Schematics of the Al decamer with a coverage of oxide layer around nanodisks. c) Scattering crosssection for different geometrical variations

3.12 Numerically calculated scattering spectral responses for the decamer cluster, while the refractive index of the environmental medium is variant. a) The position of the FRs to the presence of $\mathrm{N}_{2}$ and $\mathrm{O}_{2}$ are evaluated. b) The position of the FRs for the presence of $\mathrm{Ar}$ and $\mathrm{CO}_{2}$ is determined. c) The position of the FRs for the presence of $\mathrm{He}$ and $\mathrm{Xe}$ is drawn

3.13 Sensitivity measurement of the plasmonic gas sensor. a) A linear plot of sensitivity for the position of plasmon resonance peaks over the refractive index variations of the ambient for an Al-based decamer. b) Linear plot for the figure of merit is depicted in the presence of various gases and quantified for the proposed compositional decamer for the presence of various gases

3.14 a) The cross-sectional snapshot of the transverse electric mode distribution in a proposed multilayer nanocluster-based sensor, while the refractive index of the medium is $n=1.44$. A low leakage of the confined field is observable between $\mathrm{SiC}$ and $\mathrm{SiO}_{2}$ layer due to high confinement of light by cluster. b) Calculated confinement factor for the multilayer structure over the thickness of $\mathrm{SiC}$ layer, while the size of nanocluster is unchanged. Also, this parameter has been compared for a structure composed of $\mathrm{Au}$ slot and without plasmon resonance property regimes

3.15 Chemical sensing properties of sensors. a) Calculated scattering spectra for the proposed nanosensor while immersing by mentioned liquids. The Fano dip red-shifts by increasing the refractive index of the medium and becomes narrower and deeper. b) Quantified FoM for the plasmonic sensor by sketching the linear fit of plasmon energy $(\Delta E)$ differences over the refractive index $(n)$ alterations

3.16 Aluminum plasmonic photodetector. a) Schematic of the plasmonic photodetector composed of $\mathrm{Al}$ nanodisk clusters deposited on GaN-sapphire substrates. The inset are the definitions for a nanodisk and a heptamer cluster with geometrical parameters. b) A top-view of the photodetector with the geometrical dimensions identification. c) The cross-sectional view of the hot electron generation and transform under the Al-based nanodisk clusters at the GaN-metal 
interface. d) Schematic band diagram for the Al-GaN interface, showing the carrier formation mechanism in the device.

3.17 The plasmon responses for the UV photodetector. a) The absorption spectra for heptamer clusters deposited in $\mathrm{GaN}$ epilayer with variant oxide thickness and without metallic heptamers. b) E-field enhancement diagram for the UV device with and without Al clusters. c) Numerically plotted absorption spectra for the oxide layer thickness as a function of incident UV beam in a heptamer nanocluster

3.18 Electrical response of plasmonic UV photodetector. a, b) Carrier concentration for the detector system without heptamers with bias $(5.0 \mathrm{~V})$, while the UV light is in OFF and ON states, respectively. c, d) Carrier concentration for the system with heptamers with bias $(5.0 \mathrm{~V})$, while the UV light is in OFF and ON states, respectively. e, f) E-field enhancement map for the device with and without clusters, while the UV light is $\mathrm{ON}$ and bias is $0.0 \mathrm{~V}$

3.19 Electrical response for the UV photodetector. a) Numerically achieved photocurrent-voltage (I-V) curves for two different oxide thicknesses of a heptamer cluster and without heptamers. The inset is the dark current-voltage (IV) curves for the non-plasmonic and plasmonic UV photodetector for two different oxide thicknesses at $\lambda=325 \mathrm{~nm}$ and $335 \mathrm{~nm}$ for $t_{o x}=2 \mathrm{~nm}$, and $4 \mathrm{~nm}$, respectively. b) Polarization-independency of the generated photocurrent (bluespheres) of the device for the polarization angle variations of the incident UV beam.....

3.20 The spectral responses for the UV detector in both non-plasmonic and plasmonic regimes, with variant $\mathrm{Al}_{2} \mathrm{O}_{3}$ thicknesses of heptamer clusters. a) Responsivity profile under $5.0 \mathrm{~V}$ applied bias. Inset is the responsivity profile for the non-plasmonic regime. b) The IQEs for different regimes of the UV detector

4.1 General spectral response of a dimer in classical and quantum models. The solid line is for the classical model and the dashed line demonstrating the nonlocal hydrodynamic model for the quantum model. The inset is a dimer antenna

4.2 a) and b) The schematic and tip view pictures of the assembly, respectively. c) Calculated and measured NTA for the capacitive coupling for the following dimensions: $a / b / c / d=10 / 25 / 10 / 35 \mu \mathrm{m}$. The inset is an SEM image of the plasmonic assembly. d) The applied electro-optical signal amplitude (mV) as a function of time delay. e) E-field map for the asymmetric assembly under longitudinal polarization excitation, respectively

4.3 Characterized and simulated NTA profiles for the asymmetric THz assembly for the presence of a) a nanodisk between gaps, b) a touching disk to the VSMBs, and c) an overlapping nanodisk. The insets are the corresponding SEM pictures 
for nanodisk variations. d), e), and f) The corresponding E-field maps for different studied regimes for the CTP mode. The charge transfer is plotted inside the numerically defined maps

4.4 a), b), c), and d) The cross-sectional E-field maps and field concentration diagrams for capacitive coupling, presence of a nontouching disk, presence of a touching disk, and presence of an overlapping disk, respectively. e) Comparative absorption profiles for the VSMBs assembly with the absence and presence of intermediate disk with various diameters. $\mathrm{f}$ ) The resistance variations as a function of the intermediate disk diameter. The inset is the CTP position as a function of conductive disk diameter

4.5 a) An artistic rendering of the proposed tunable dimer nanoantenna on a glass substrate. b) A top-view plot of the dimer nanoantenna with corresponding geometrical parameters

4.6 a) Normalized extinction spectra of the bridged dimer in GST-mediated, fullmetallic, and air regimes. The inset is the normalized extinction cross-section for the same dimer without conductive junction and plotted for the capacitive coupling regime (the offset gap between nanoparticles is $15 \mathrm{~nm}$ ). b), c) E-field maps across the bridged dimer for dipolar and CTP resonant mode in full metallic regime, respectively, for both (i) top-view and (ii) cross-sectional views. d), e) The local E-field distributions correlating with the dipolar and CTP resonant peaks, respectively, for both (i) top-view and (ii) cross-sectional views. (iii) The electric-field intensity diagrams $|E|^{2}$ for the metallodielectric and metallic dimers at the position of CTP and dipolar modes.....

4.7 a), (b), (c), and (d): (i) Normalized extinction spectra for the GST-mediated bridged dimer, while the $L_{G S T}$ is variant. (ii) and (iii) The corresponding E-field maps for $L_{G S T}$ variations for both amorphous and crystalline phases

4.8 a), (b), (c) and (d): Normalized extinction profiles for the GST-mediated bridge dimer antenna with different bridge thickness $(W)$. (e) and (f) The FWHM profiles as a function of $L_{G S T}$ and $W$ for the presence of both a-GST and c-GST layers at the center of bridge

4.9 The transmission ratio of the GST-mediated metallodielectric switch in OFF (amorphous) and ON (crystalline) states

4.10 a) An artistic rendering of the proposed device composed of gold nanodisks and graphene monolayer. b) A top-view of the device showing the corresponding geometrical parameters. c) Electrical transport characteristic curves as resistance variations $(R)$ as a function of back-gate voltage $(V g)$ for the proposed grapheneplasmonic platform. The diameter of nanodisks is $d=125 \mathrm{~nm}$ and the offset space between them is $L_{a}=100 \mathrm{~nm}$ while the thickness is $t=45 \mathrm{~nm}$ for both. The distance 
between source-drain electrodes is $L_{g}=500 \mathrm{~nm}$ and the width of graphene sublayer is $W=160 \mathrm{~nm}$

4.11 a) Extinction cross-section of a highly-doped to $E_{F}=0.61 \mathrm{eV}$ (n-type) graphene nanoribbon under optical excitation at room-temperature $T=300 \mathrm{~K}$. The inset is the E-field intensity map for the dipolar mode. b) Extinction spectra of plasmonic nanodimer antenna on the highly doped graphene nanoribbon bridge, supporting dipolar (II), CTP (III), quadrupolar (IV), and dipolar (V) moments. c) The local $|\mathrm{E}|$-field intensity snapshots for the plasmon resonance excitation and distribution across the device for dipole (II) and CTP (III) modes. d) The charge density plots for the CTP spectral feature and dipolar modes

4.12 a) Normalized extinction spectra for interparticle distance (50 $\mathrm{nm} \leq L_{a} \leq 120$ $\mathrm{nm})$ and $\mathrm{b})$ nanodisks diameters $(75 \mathrm{~nm} \leq d \leq 150 \mathrm{~nm})$ variations, respectively. The doping of graphene is n-type Fermi level energy if fixed to $E_{F}=0.61 \mathrm{eV}$

4.13 Normalized extinction spectra for the "On" and "Off" states of the grapheneplasmonic nanoswitch in the presence and absence of back-gate voltage. The critical geometrical parameters are: $L_{a}=100 \mathrm{~nm}, d=120 \mathrm{~nm}$. The inset is the local E-field map for the dipolar resonance, when the graphene sublayer is in semiconducting regime

4.14 The CTP energy as a function of gate voltage variations $\left(\Delta V_{g}\right)$. The insets are the extinction diagram for the plasmonic nanoswitch for the gate voltage variations below the Dirac point and the $Q$-factor of CTP peak for the gate voltage variations

4.15 Numerically quantified MD of the graphene-plasmonic switch as a function of incident photon wavelength with higher resolution under back-gate bias application (at the Dirac point)

5.1 Schematic for the EM dipoles ( $\mathbf{p}$ and $\mathbf{m}$ ) and unconventional toroidal dipoles (T), Ref. [14], obtained with permissions from APS

5.2 a) Graphical representation of bimetallic plasmonic metamolecules on a silicon host under $\mathrm{THz}$ beam illumination. b) An introduction to the geometrical components of unit cell. c) The SEM image of fabricated plasmonic multipixel structures in arrays with the gap spots between surrounding and central resonators of $D_{g}=3 \mu \mathrm{m}$, while the other geometries are fixed to: $L=240 \mu \mathrm{m}, R=50 \mu \mathrm{m}$, $W_{l}=30 \mu \mathrm{m}$, and $W_{2}=40 \mu \mathrm{m}$

$5.3 \mathrm{a}$ ), b) Experimentally and numerically defined transmission amplitudes for the plasmonic metamolecule with different gap distances, respectively. c) An artistic illustration for the formation of toroidal moment and circulation of closed-loop 
current. d), e) The local H-field map for the localization of plasmons at the toroidal frequency, and surface current density plot for the magnetic current oscillation across the antenna.

5.4 a) Experimentally measured normalized transmission amplitude for both toroidal and magnetic responses of the plasmonic unit cell under different magnetic polarization angles $0^{\circ} \leq \varphi \leq 90^{\circ}$. b) Toroidal response of the unit cell as a function of incident $\mathrm{THz}$ radiation magnetic component angle. c) The MD percentage as a function of $D_{g}$, showing the highest value around $\sim 96 \%$. d) The polar plot for both experimentally and numerically obtained transmission spectra at the position of the toroidal mode

5.5 Fano resonant unit cell with the geometrical dimensions as follows: $\left(R_{o} / R_{i} / D_{g} / W / L\right)=(95 / 65 / 10 / 70 / 325) \mathrm{nm}$. a) Schematic and b) top-view profiles for the plasmonic unit cell. c) Normalized amplitude transmission spectra for the Fano resonant structure. The inset is a rendering for the coherent oscillation of charges. d) Normalized absorption and reflection spectra for the proposed unit cell. e), f) The local E-field maps for the plasmon resonance excitation at the Fano dip and bright mode positions, respectively. g) The surface current of resonator at narrow Fano dip wavelength

5.6 Toroidal resonant unit cell. a) Schematic and b) top-view profiles for the plasmonic unit cell. c) Normalized amplitude transmission spectra for the toroidal structure. The inset is a rendering for the opposite oscillation of magnetic moments, showing formation of a closed-loop current. d) The local E-field maps for the plasmon resonance excitation at the toroidal dipole position. e) and f) The surface current of resonator at toroidal and magnetic dipole wavelengths, respectively

5.7 The $y z$-plane for the magnetic field $(|\mathrm{H}|$-field $)$ excitation and direction across the unit cell for (a) Fano dip and (b) toroidal dipole.....

5.8 (a) Transmission spectra for the toroidal resonator for samples having different arc lengths $\left(L_{\text {arc }}\right)$. (b) and (c) The surface current of resonator at toroidal dipole position for two different arc lengths, 104 and $174 \mathrm{~nm}$, respectively. (d) and (e) The surface current of resonator at magnetic dipole moment for two different arc lengths, 104 and $174 \mathrm{~nm}$, respectively

5.9 a) Scattering powers of toroidal and magnetic moments $\left(L_{a r c}\right)$. (b) The scattering power as a function of arc length variations for toroidal and magnetic moments.....

5.10 A schematic for the metallodielectric unit cell with GST arcs. b) and c) The transmission spectra for the arc length variations in a-GST and c-GST states of arcs, respectively. d) and e) The E-field maps for the metallodielectric unit cell in 
c-GST and a-GST phases of arcs, respectively at the Fano and toroidal wavelengths. f) and g) The surface current density for the c-GST and a-GST states of arcs, respectively

5.11 Transmission spectra for the toroidal dipole for (a) inner radius, (b) outer radius, and (c) gap distance variations....

5.12 Transmission spectra for the Fano dip for (a) inner and other radii, (b) gap distance, (c) and (d) length and width of the central bar variations, respectively

5.13 Transmission ratio for the GST-mediated metallodielectric unit cell in both a-GST and c-GST states, resembling an NIR plasmonic switch

6.1 a, b, c) Schematic diagrams for an $\mathrm{Ag}$ nanoparticle and a four-member quadrumer composed of nanoparticles in both metallic and metallodielectric regimes, respectively. d, e) Scattering and absorption profiles for the metallic quadrumer in the free space and aqueous ambiences under transverse polarization excitation $\left(E_{\text {exc }}\right)$. The insets show the gap distance between core-shell units and Efield illumination direction. $\mathrm{f}, \mathrm{g}$ ) E-field profiles during plasmon resonance excitations coupling in the metallic quadrumer in free space and liquid systems

6.2 a) Thermal heat power flux $\left(Q_{h}\right)$ profile for a metallic nanoassembly during laser pump exposure in a liquid system. b) Dissipated power density mapping in a metallic quadrumer at the peak of absorption

$6.3 \mathrm{a}, \mathrm{b})$ Scattering and absorption spectra for quadrumer cluster with the carbon nanospheres with different quantities in an aqueous system. c, d) The electric field profile $|\mathrm{E}|$ showing the hybridization and enhancement in metallodielectric quadrumer clusters with two and four CNSs, respectively. e, f) The electric field distribution diagram inside the metallodielectric quadrumer with four carbon nanospheres at Fano dip position for bright and dark modes. Carbon nanospheres are denoted by CNS in the picture

6.4 a) Field enhancement factor $\left(\left|E_{\text {in }}\right| /\left|E_{\text {exc }}\right|\right)$ over the photon energy for both metallic and metallodielectric (with four CNSs) clusters in an aqueous ambience. b) Photothermal heat power flux $\left(Q_{h}\right)$ spectra at the interparticle junction for a metallodielectric assembly with CNSs. c) Photothermal heat density in quadrumer with four CNSs. d) Photothermal heat temperature variations over the laser pulse intensity for both metallic and metallodielectric plasmonic clusters. Carbon spheres are denoted by CNS in the picture

7.1 Contribution of the ZIKV external morphology and the cryo-electron microscopic image of hydrated ZIKV. a) An artistic representation of the ZIKV virion structure. b) The cross-section of cryo-electron microscopic illustration of 
ZIKV, showing the radial density distribution with specific color coding, obtained with permissions from AAAS [10]

7.2 Point-of-care molecular detector. a) Schematic of saliva sample preparation. Saliva samples are first collected in a saliva collection tube and then lysed in Qiagen binding/ lysis (AVL) buffer. b) The lysed sample is filtered through the isolation membrane of our microfluidic cassette for nucleic acid extraction. c) Exploded view of the chemically heated cup. The cup consists of a thermos cup body, a 3D-printed cup lid, a chip holder, PCM material, heat sink and single-use $\mathrm{Mg}-\mathrm{Fe}$ alloy pack heat source. d) A photograph of the chemically heated cup for point of care molecular diagnostics of ZIKV, obtained with permission from ACS $[11]$

7.3 Toroidal plasmonic metamolecule. a) Artistic perspective of compositional plasmonic resonators assembly as a unit cell on a silicon host. b) A top-view schematic of the microstructure unit cell with an introduction to geometrical components. c) The SEM image of fabricated plasmonic structures in arrays for the unit cells with the gap spots between surrounding and central resonators of $D_{g}=3 \mu \mathrm{m}$ with $L=240 \mu \mathrm{m}, R=50 \mu \mathrm{m}, W_{l}=30 \mu \mathrm{m}$, and $W_{2}=40 \mu \mathrm{m}$. d) The magnified SEM images for each unit cell with $D_{g}=3 \mu \mathrm{m}$.

$7.4 \mathrm{a})$ and $\mathrm{b}$ ) The 3D schematics of the magnetic (m) and toroidal (T) resonances around and across the central and surrounding magnetic resonators, respectively. c), d), and e) The electromagnetic response of the compositional $\mathrm{THz}$ plasmonic resonator: (i) Experimentally obtained normalized transmission amplitude profiles for the unit cells with three different offset gaps, (ii) the SEM images for different offset gaps between resonators, (iii) Numerically calculated transmission spectra for three different offset gaps

7.5 The electromagnetic response of the plasmonic unit cell at a) toroidal and b) magnetic resonance frequencies. Numerically obtained local $|\mathrm{H}|$-field $(\mathrm{A} / \mathrm{m})$ snapshots for the toroidal and magnetic resonance confinement and excitation at the gap spot area between resonators in (i) linear and (ii) logarithmic scales. (iii) The cross-sectional vectorial maps for the magnetic field lines at the position of toroidal and magnetic resonances. c) Simulated surface currents of unit cell at toroidal and magnetic resonances

7.6 Normalized transmission amplitude profiles of the THz resonator system with three different offset gaps obtained (i) experimentally and (ii) numerically for (a) $D_{g}=3 \mu \mathrm{m}$, (b) $D_{g}=4 \mu \mathrm{m}$, and (c) $D_{g}=5 \mu \mathrm{m}$. The insets are the SEM images with the geometrical parameters

7.7 a) Schematic demonstration of ZIKV envelope protein binding with respective antibody on the toroidal THz plasmonic metasurface. b), and c) The SEM images 
for the plasmonic toroidal resonator covered with antibody and ZIKV envelope proteins attached to the antibody, respectively

7.8 Transmission spectra for the toroidal resonant mode behavior for presence of different concentration of ZIKV envelope protein from (i) antibody to $50 \mathrm{pg} / \mathrm{mL}$ and (ii) $100 \mathrm{pg} / \mathrm{mL}$ to $10^{4} \mathrm{pg} / \mathrm{mL}$

7.9 a) Toroidal resonance frequency shifts due to conjugated ZIKV protein concentration (solid) and fitting line (dotted). b) The transmission spectra for a $\mathrm{THz}$ plasmonic chip characterized for three days to define the repeatability of a sample

8.1 a) Artistic rendering of the toroidal unit cell. b) Geometrical parameters of the unit cell. c) SEM image of the fabricated metasurface. (d) Schematics of the formation of head-to-tail arrangement correlating with the toroidal momentum between the proximal resonators with the direction of magnetic momenta. e) A schematic representation of the BWO setup used to characterize the spectral response of the metasensor

8.2 a), b), and c) Normalized transmission amplitude for the toroidal metamaterials with three different gap distances $g=7 \mu \mathrm{m}, 5 \mu \mathrm{m}$, and $3 \mu \mathrm{m}$, respectively. d) Local near-field map of the E-field enhancement at the gaps at the toroidal mode frequency. e) Surface current plot for the current across the structure and formation of toroidal mode. f) A cross-sectional $y z$-plane of the resonators, showing the head-to-tail magnetic moments forming the toroidal moment in a vectorial map.

8.3 Surface current as a function of $x$-axis for three different gap spacing. b) The toroidal scattering intensity as a function of frequency for three different gap spacing. c) The quality factor of the toroidal lineshape and dephasing time $(\tau)$ as a function of three different gap spacing

8.4 a) Schematic flowchart of functionalized gold nanoparticle conjugation with the ZIKV antibody and ZIKV-envelope proteins (NS1) with the explanation for different parts. b) Schematic representation of gold nanoparticles-integrated toroidal unit cells. c) and d) The SEM images of plasmonic metamolecule in the presence of GNPs with antibody and ZIKV envelop proteins, respectively

8.5 a), b) TEM images of functionalized GNPs binding with antibody in two different scales. c) The TEM image of ZIKV envelope proteins captured by antibody-conjugated GNPs. In this picture, the AB stands for antibody...

8.6 a) The transmission amplitude spectra for the fabricated metasurfaces in both $\mathrm{W} /$ and W/O GNPs regimes in the presence of ZIKV antibody and ZIKV envelope proteins with different concentrations. (b) The toroidal resonance shift 
as a function of ZIKV envelope proteins concentration W/ and W/O GNPs with the corresponding determination coefficient. (c) The magnified transmission spectra as a function of frequency, showing the maximum shift of the toroidal moment in the presence and absence of GNPs attached to the system.........................214

8.7 a) and (b) The toroidal resonance shift $(\Delta \omega)$ and quality factor as a function to time in hours for the presence and absence of GNPs 


\section{LIST OF ACRONYMS \& ABBREVATIONS}

$\mathrm{THz} \quad$ Terahertz

CTP Charge Transfer Plasmons

EM Electromagnetic

Rh Rhodium

Ga Gallium

Cr Chromium

In Indium

Au Gold

Ag Silver

$\mathrm{Cu} \quad$ Copper
Al Aluminum
$\mathrm{Ni} \quad$ Nickel
Ti Titanium
$\mathrm{Mg}$ Magnesium
CdSe Cadmium Selenide
GaN Gallium Nitride
$\mathrm{ZnO}$ Zinc Oxide
$\mathrm{VO}_{2} \quad$ Vanadium Dioxide
GST $\mathrm{Ge}_{2} \mathrm{Sb}_{2} \mathrm{Te}_{5}$
UV Ultraviolet
NIR Near-Infrared
MIR Mid-Infrared
FIR Far-Infrared
SERS Surface-Enhanced Raman Spectroscopy
SPRs Surface Plasmon Resonances 
TM Transverse Magnetic

TE Transverse Electric

FDTD Finite-Difference Time-Domain

FE Finite-Elements

DGTD Discontinuous Galerkin Time-Domain Method

TDDFT Time-Dependent Density Functional Theory

VIM Volume Integral Method

PML Perfectly Matched Layer

PMC Perfect Magnetic Conductor

LSPR Localized Surface Plasmon Resonances

DUV Deep Ultraviolet

FoM Figure of Merit

MFP Mean-Free Path

IQE Internal Quantum Efficiency

EQE External Quantum Efficiency

AFM Atomic Force Microscope

QCM Quantum Corrected Model

THz-TDS THz Time-Domain Spectrometer

BWO Backward Wave Oscillator

NTA Normalized Transmission Amplitude

PCM Phase-Change Materials

NVRAM Nonvolatile Random Access Memory

a-GST Amorphous GST

c-GST Crystalline GST

EBL Electron-Beam Lithography

MD Modulation Depth 
ZIKV Zika-Virus

ELISA Enzyme-Linked Immunosorbent Assay

LCV leuco crystal violet

GNP Gold Nanoparticles

OD Optical Density

SEM Scanning Electron Microscopy

TEM Transmission Electron Microscopy

LoD Limit of Detection

DI Deionized

MSM Metal-Semiconductor-Metal

FWHM Full Wave at Half Maximum

RPA Random Phase Approximation

$\mathrm{kDa} \quad$ Kilo Dalton 


\section{CHAPTER 1}

\section{Introduction}

\subsection{Plasmonics}

The study of the interaction between an intense electromagnetic (EM) field and free electrons in the $d$-band of subwavelength metallic components is well-known as "surface plasma oscillations" [1-4]. The existence of these collective, coherent and evanescent fluctuations of optically excited electrons at the metal-dielectric interfaces has been demonstrated successfully using several practical methods such as electron energy-loss experiments [6-8], excitation by electrons-matter interaction [1,9], light-matter interaction [1-4,10-12], and electrical excitation $[13,14]$. As one of the fundamental techniques, light-matter interaction has been acknowledged as a promising and practical approach for the excitation of dynamic surface plasmon polaritons in the subwavelength regime. The optical excitation of plasmons strongly depends on the light intensity, material, and geometry of the components $[15,16]$. The electrical component of the incident wave plays the major role in the induction of free electrons in metals [4]. On the other hand, noble metals (i.e. gold $(\mathrm{Au})$, silver $(\mathrm{Ag})$, copper $(\mathrm{Cu})$, and aluminum $(\mathrm{Al})$ ) are the substances that have widely been employed for excitations of surface plasmons and development of plasmonic structures and devices for a couple of decades [17-19].

\subsection{Motivation}

As a key counterpart of photonics, plasmonics is of interest to various fields of researches, because of having a broad range of applications extending from ultraviolet (UV) to the far-infrared (FIR) and terahertz (THz) spectra [2,20]. Plasmonic structures 
are being explored for their significant potential for tailoring several optic-based devices and platforms including but limited to telecommunication nanophotonic devices [21-27], light harvesters and solar cells [28-32], biochemical and environmental sensors and transducers [33-36], transistors and photodetectors [37-40], metamaterials [41-44] advanced medical surgery tools $[45,46]$, tumor and cancer therapies $[47,48]$, photothermal heat generation [49,50], bubble formation for surgery [51], DNA detection [52], nonlinear optics [53,54], and surface-enhanced Raman spectroscopy (SERS) applications $[52,55]$. Although plasmonics facilitates the development of various efficient and practical devices, these devices suffer from both radiative and nonradiative losses [15]. To address such an inherent shortcoming, several strategies have been carried out to reduce the lossy behavior and increase the efficiency and responsivity of plasmonic devices that will be discussed in the following chapters [56].

\subsection{Scope}

The major objective of this dissertation is to investigate novel subwavelength plasmonic structures to support ultrastrong resonances along the UV to the THz spectrum. Using the optically excited moments, we tailored and analyzed nanoplasmonic devices for various applications from sensing to switching. By underlying the physics behind the formation of fundamental and famous plasmonic resonances such as Fano lineshape, charge transfer plasmons (CTP), and toroidal moments, we employed these structures to develop efficient, cost-effective, functional, and practical advanced nanophotonic and bio-related devices. Using all of the mentioned resonant moments for developing optical and optoelectronic nano and microscale devices, we numerically and experimentally studied the plasmonic response of several devices for real nanophotonic applications. 
This work also describes the biomedical applications of plasmonic moments and their advantages in biosensing applications. As promising platforms, this dissertation also focuses on the utilization of toroidal $\mathrm{THz}$ plasmonic metamaterials to design optical sensors for the detection of nanoscale and low molecular-weight infectious biomarkers. Introducing various structures and platforms, the exquisite properties of toroidal plasmonic metasurfaces for the detection of the presence of Zika-virus envelope proteins is discussed comprehensively with experimental demonstrations. This work also explains the methods and techniques to enhance the detection quality, sensitivity, repeatability, and reliability of toroidal $\mathrm{THz}$ plasmonic metasurfaces.

\subsection{Organization of the Dissertation}

The rest of the dissertation is organized as follows: Chapter 2 provides a brief review of the theory of surface plasmons excitation and the physics behind the formation of electron oscillations at the metal-dielectric interface in subwavelength regime. This chapter also covers the conventional numerical methods for analyzing the plasmonic response of various devices and nanostructures. The unique result of light-matter interaction is the formation of EM modes, described in Chapter 3. The spectral properties of various nanoscale structures based on both classical near-field coupling and stronglycoupled (hybridized of plasmons) systems are expressed in details. Chapter 4 provides the plasmonic behavior of nanoscale objects located in subnanometer gap spots, characterized by quantum mechanical principle. In addition, this chapter focuses on the direct transfer of optically driven charges in jointed nanoparticles. Furthermore, the possibility of finely tuning the excited spectral features in these regimes are discussed in this chapter and the proposed solutions are explained using numerical tools. A new 
family of EM multipoles, known as toroidal moments are explained in Chapter 5. This chapter introduces the theory of the excitation of dipolar and multipolar toroidal modes along the NIR to the $\mathrm{THz}$ band, and the solutions to optimize the tunability of these moments are explained comprehensively. By demonstrating the numerically analyzed and experimentally defined spectral properties of our tailored structures, this chapter helps to introduce the toroidal moments as fundamental resonances for developing advanced nanophotonic devices and biomedical-related platforms.

In Chapter 6, the advantages of utilizing plasmonic structures in biomedical and clinical applications are reviewed and discussed. The generation of photothermal heat flux in Fano-resonant plasmonic assemblies is explained here. Finally, Chapters 7 and 8 focus on the use of toroidal plasmonic metasurfaces for advanced biosensing applications. The simulations are followed by experimental investigations to define the sensitivity of toroidal $\mathrm{THz}$ plasmonic metamaterials for the detection of low-molecular weight infectious biomarkers and the methods for the optimization of sensors. Taking the advantage of the non-invasive and non-poisonous features of $\mathrm{THz}$ beam technology and also by employing the narrowness of the resonant lineshape of the toroidal plasmonic multipoles, we successfully provided label-free, real-time, room-temperature, fast, costeffective, promising, and accurate plasmonic metasensors as leading structures for advanced biosensing purposes. Chapter 9 concludes the work and provides a summary of the works that have been done and also provides a view for the possible future works that can be done using the proposed nano and microdevices. 


\subsection{References}

1. Raether, H. (1988). Surface plasmons on smooth and rough surfaces and on gratings (Vol. 1). Springer, Berlin, Heidelberg.

2. Barnes, W. L., Dereux, A., \& Ebbesen, T. W. (2003). Surface plasmon subwavelength optics. Nature, 424(6950), 824-830.

3. Ozbay, E. (2006). Plasmonics: merging photonics and electronics at nanoscale dimensions. Science, 311(5758), 189-193.

4. Maier, S. A. (2007). Plasmonics: fundamentals and applications (Vol. 1). Springer Science \& Business Media.

5. Raether, H. (1982). Dispersion relation of surface plasmons on gold-and silver gratings. Optics Communications, 42(4), 217-222.

6. Stern, E. A., \& Ferrell, R. A. (1960). Surface plasma oscillations of a degenerate electron gas. Physical Review, 120(1), 130.

7. Ritchie, R. H., \& Marusak, A. L. (1966). The surface plasmon dispersion relation for an electron gas. Surface Science, 4(3), 234-240.

8. Powell, B. D., \& Woodruff, D. P. (1972). Plasmon effects in electron energy loss and gain spectra in aluminum. Surface Science, 33(3), 437-444.

9. Raether, H. (2006). Excitation of plasmons and interband transitions by electrons (Vol. 88). Springer, Berlin, Germany.

10. Hecht, B., Bielefeldt, H., Novotny, L., Inouye, Y., \& Pohl, D. W. (1996). Local excitation, scattering, and interference of surface plasmons. Physical Review Letters, 77(9), 1889.

11. Sambles, J. R., Bradbery, G. W., \& Yang, F. (1991). Optical excitation of surface plasmons: an introduction. Contemporary Physics, 32(3), 173-183.

12. Pendry, J. B., Martin-Moreno, L., \& Garcia-Vidal, F. J. (2004). Mimicking surface plasmons with structured surfaces. Science, 305(5685), 847-848.

13. Bharadwaj, P., Bouhelier, A., \& Novotny, L. (2011). Electrical excitation of surface plasmons. Physical Review Letters, 106(22), 226802.

14. Neutens, P., Lagae, L., Borghs, G., \& Van Dorpe, P. (2010). Electrical excitation of confined surface plasmon polaritons in metallic slot waveguides. Nano Letters, 10(4), 1429-1432. 
15. Maier, S. A., Brongersma, M. L., Kik, P. G., Meltzer, S., Requicha, A. A., \& Atwater, H. A. (2001). Plasmonics - a route to nanoscale optical devices. Advanced Materials, 13(19), 1501-1505.

16. Maier, S. A. (2006). Plasmonics: The promise of highly integrated optical devices. IEEE Journal of selected topics in Quantum Electronics, 12(6), 1671-1677.

17. Schuchinsky, A. G. (2010). Surface plasmons in noble metal films. Metamaterials, 4(2), 61-69.

18. Knight, M. W., King, N. S., Liu, L., Everitt, H. O., Nordlander, P., \& Halas, N. J. (2013). Aluminum for plasmonics. ACS Nano, 8(1), 834-840.

19. Ahmadivand, A., \& Golmohammadi, S. (2015). Surface plasmon resonances and plasmon hybridization in compositional $\mathrm{Al} / \mathrm{Al}_{2} \mathrm{O}_{3} / \mathrm{SiO}_{2}$ nanorings at the $\mathrm{UV}$ spectrum to the near infrared region (NIR). Optics \& Laser Technology, 66, 9-14.

20. Dragoman, M., \& Dragoman, D. (2008). Plasmonics: Applications to nanoscale terahertz and optical devices. Progress in Quantum Electronics, 32(1), 1-41.

21. Noual, A., Akjouj, A., Pennec, Y., Gillet, J. N., \& Djafari-Rouhani, B. (2009). Modeling of two-dimensional nanoscale Y-bent plasmonic waveguides with cavities for demultiplexing of the telecommunication wavelengths. New Journal of Physics, 11(10), 103020.

22. Ahmadivand, A., Golmohammadi, S., \& Rostami, A. (2012). T-and Y-splitters based on an $\mathrm{Au} / \mathrm{SiO}_{2}$ nanoring chain at an optical communication band. Applied Optics, 51(15), 2784-2793.

23. Ahmadivand, A., Golmohammadi, S., \& Rostami, A. (2013). Broad comparison between $\mathrm{Au}$ nanospheres, nanorods and nanorings as an S-bend plasmon waveguide at optical C-band spectrum. Journal of Optical Technology, 80(2), 80-87.

24. Melikyan, A., Alloatti, L., Muslija, A., Hillerkuss, D., Schindler, P.C., Li, J., Palmer, R., Korn, D., Muehlbrandt, S., Van Thourhout, D. and Chen, B. (2014). High-speed plasmonic phase modulators. Nature Photonics, 8(3), 229-233.

25. Ahmadivand, A., \& Golmohammadi, S. (2014). Comprehensive investigation of noble metal nanoparticles shape, size and material on the optical response of optimal plasmonic Y-splitter waveguides. Optics Communications, 310, 1-11.

26. Ahmadivand, A. (2014). Hybrid photonic-plasmonic polarization beam splitter (HPPPBS) based on metal-silica-silicon interactions. Optics \& Laser Technology, $58,145-150$.

27. Haffner, C., Heni, W., Fedoryshyn, Y., Niegemann, J., Melikyan, A., Elder, D.L., Baeuerle, B., Salamin, Y., Josten, A., Koch, U. and Hoessbacher, C. (2015). All- 
plasmonic Mach-Zehnder modulator enabling optical high-speed communication at the microscale. Nature Photonics, 9(8), 525-528.

28. Pillai, S., Catchpole, K. R., Trupke, T., \& Green, M. A. (2007). Surface plasmon enhanced silicon solar cells. Journal of Applied Physics, 101(9), 093105.

29. Ferry, V. E., Sweatlock, L. A., Pacifici, D., \& Atwater, H. A. (2008). Plasmonic nanostructure design for efficient light coupling into solar cells. Nano Letters, 8(12), 4391-4397.

30. Catchpole, K. R., \& Polman, A. (2008). Plasmonic solar cells. Optics Express, 16(26), 21793-21800.

31. Ferry, V.E., Verschuuren, M.A., Li, H.B., Verhagen, E., Walters, R.J., Schropp, R.E., Atwater, H.A. and Polman, A., 2010. Light trapping in ultrathin plasmonic solar cells. Optics Express, 18(102), A237-A245.

32. Ahmadivand, A., \& Pala, N. (2017). Absorption enhancement in ultrathin structures based on crystalline-Si/Ag parabola nanocones periodic arrays with broadband antireflection property. Silicon, 9(1), 25-29.

33. Anker, J. N., Hall, W. P., Lyandres, O., Shah, N. C., Zhao, J., \& Van Duyne, R. P. (2008). Biosensing with plasmonic nanosensors. Nature Materials, 7(6), 442-453.

34. Lassiter, J. B., Sobhani, H., Fan, J. A., Kundu, J., Capasso, F., Nordlander, P., \& Halas, N. J. (2010). Fano resonances in plasmonic nanoclusters: geometrical and chemical tunability. Nano Letters, 10(8), 3184-3189.

35. Golmohammadi, S., \& Ahmadivand, A. (2014). Fano resonances in compositional clusters of aluminum nanodisks at the UV spectrum: a route to design efficient and precise biochemical sensors. Plasmonics, 9(6), 1447-1456.

36. Caucheteur, C., Guo, T., \& Albert, J. (2015). Review of plasmonic fiber optic biochemical sensors: improving the limit of detection. Analytical and Bioanalytical Chemistry, 407(14), 3883-3897.

37. Lin, J., Li, H., Zhang, H., \& Chen, W. (2013). Plasmonic enhancement of photocurrent in MoS2 field-effect-transistor. Applied Physics Letters, 102(20), 203109.

38. Fang, Z., Liu, Z., Wang, Y., Ajayan, P. M., Nordlander, P., \& Halas, N. J. (2012). Graphene-antenna sandwich photodetector. Nano Letters, 12(7), 3808-3813.

39. Gogurla, N., Sinha, A. K., Santra, S., Manna, S., \& Ray, S. K. (2014). Multifunctional Au-ZnO plasmonic nanostructures for enhanced UV photodetector and room temperature NO sensing devices. Scientific Reports, 4, 6483. 
40. Ahmadivand, A., Sinha, R., Vabbina, P. K., Karabiyik, M., Kaya, S., \& Pala, N. (2016). Hot electron generation by aluminum oligomers in plasmonic ultraviolet photodetectors. Optics Express, 24(12), 13665-13678.

41. Henzie, J., Lee, M. H., \& Odom, T. W. (2007). Multiscale patterning of plasmonic metamaterials. Nature Nanotechnology, 2(9), 549-554.

42. Boltasseva, A., \& Atwater, H. A. (2011). Low-loss plasmonic metamaterials. Science, 331(6015), 290-291.

43. Yao, K., \& Liu, Y. (2014). Plasmonic metamaterials. Nanotechnology Reviews, 3(2), 177-210.

44. Ahmadivand, A., Karabiyik, M., \& Pala, N. (2015). Fano-like resonances in split concentric nanoshell dimers in designing negative-index metamaterials for biologicalchemical sensing and spectroscopic purposes. Applied Spectroscopy, 69(5), 563-573.

45. Brzobohatý, O., Šiler, M., Trojek, J., Chvátal, L., Karásek, V., Paták, A., Pokorná, Z., Mika, F. and Zemánek, P. (2015). Three-dimensional optical trapping of a plasmonic nanoparticle using low numerical aperture optical tweezers. Scientific Reports, 5, 8106.

46. Lu, Y., Du, G., Chen, F., Yang, Q., Bian, H., Yong, J., \& Hou, X. (2016). Tunable potential well for plasmonic trapping of metallic particles by bowtie nano-apertures. Scientific Reports, 6, 32675.

47. Li, Y., Wen, T., Zhao, R., Liu, X., Ji, T., Wang, H., Shi, X., Shi, J., Wei, J., Zhao, Y. and $\mathrm{Wu}, \mathrm{X}$. (2014). Localized electric field of plasmonic nanoplatform enhanced photodynamic tumor therapy. ACS Nano, 8(11), 11529-11542.

48. Mackey, M. A., Ali, M. R., Austin, L. A., Near, R. D., \& El-Sayed, M. A. (2014). The most effective gold nanorod size for plasmonic photothermal therapy: theory and in vitro experiments. The Journal of Physical Chemistry B, 118(5), 1319-1326.

49. Coppens, Z. J., Li, W., Walker, D. G., \& Valentine, J. G. (2013). Probing and controlling photothermal heat generation in plasmonic nanostructures. Nano Letters, 13(3), 1023-1028.

50. Ahmadivand, A., Pala, N., \& Güney, D. Ö. (2015). Enhancement of photothermal heat generation by metallodielectric nanoplasmonic clusters. Optics Express, 23(11), A682-A691.

51. Baffou, G., Polleux, J., Rigneault, H., \& Monneret, S. (2014). Super-heating and micro-bubble generation around plasmonic nanoparticles under cw illumination. The Journal of Physical Chemistry C, 118(9), 4890-4898. 
52. Ngo, H. T., Wang, H. N., Fales, A. M., \& Vo-Dinh, T. (2016). Plasmonic SERS biosensing nanochips for DNA detection. Analytical and Bioanalytical Chemistry, 408(7), 1773-1781.

53. Lan, S., Kang, L., Schoen, D. T., Rodrigues, S. P., Cui, Y., Brongersma, M. L., \& Cai, W. (2015). Backward phase-matching for nonlinear optical generation in negative-index materials. Nature Materials, 14(8), 807.

54. Gerislioglu, B., Ahmadivand, A., \& Pala, N. (2017). Hybridized plasmons in graphene nanorings for extreme nonlinear optics. Optical Materials, 73, 729-735.

55. Hugall, J. T., \& Baumberg, J. J. (2015). Demonstrating photoluminescence from Au is electronic inelastic light scattering of a plasmonic metal: the origin of SERS backgrounds. Nano Letters, 15(4), 2600-2604.

56. Khurgin, J. B. (2015). How to deal with the loss in plasmonics and metamaterials. Nature Nanotechnology, 10(1), 2-6. 


\section{CHAPTER 2}

\section{Fundamental of Plasmonics}

Here, we describe the fundamentals, theory and existing and potential applications of surface plasmon resonances (SPR) that are required for better understaning the operation of the structures and devices presented in the rest of this dissertation. This chapter also provides an introduction to surface plasma fluctuations and a summary of their properties in metallic components, as well as the numerical approaches to analyze the spectral response of subwavelength plasmonic structures.

\subsection{Theory}

To understand the properties of SPRs in various subwavelength structures, one needs to understand the spectral response of noble metals. In a wide range of frequencies, the optical properties of metals can be explained by a plasma model. In this model, a free electron gas moves against fixed positive ion cores, while the electron-electron interactions are ignored. The corresponding response of the free electron gas to the applied electric field describes the optical properties of metals. Coherent oscillation of optical driven electrons is damped due to electron-core collisions with a characteristic collision frequency $\gamma=1 / \tau$ of the order of $100 \mathrm{THz}$, where $\tau$ is mean free time of free electron gas, which is of the order of $10^{-14} \mathrm{~s}$ at room temperature. By using the Drude model for metals, one can obtain the complex permittivity of metals as a function of frequency in the following form [1]:

$$
\varepsilon(\omega)=1-\frac{\omega_{p}^{2}}{\omega^{2}\left(\frac{i}{\omega \tau}+1\right)}
$$


where $\omega_{p}$ is the plasmon frequency of the corresponding bulk metal. The Drude free electron model of metals considers the valence electrons of the atoms to be free. When an electric field is applied, the free electrons accelerate and then undergo collisions with the characteristic scattering time [2]. By assuming the system is a loss-less medium, and neglecting the collision and scattering parameters, then:

$$
\varepsilon(\omega)=1-\frac{\omega_{p}^{2}}{\omega^{2}}
$$

Consequently, the permittivity is negative when the frequency is lower than $\omega_{p}$. A

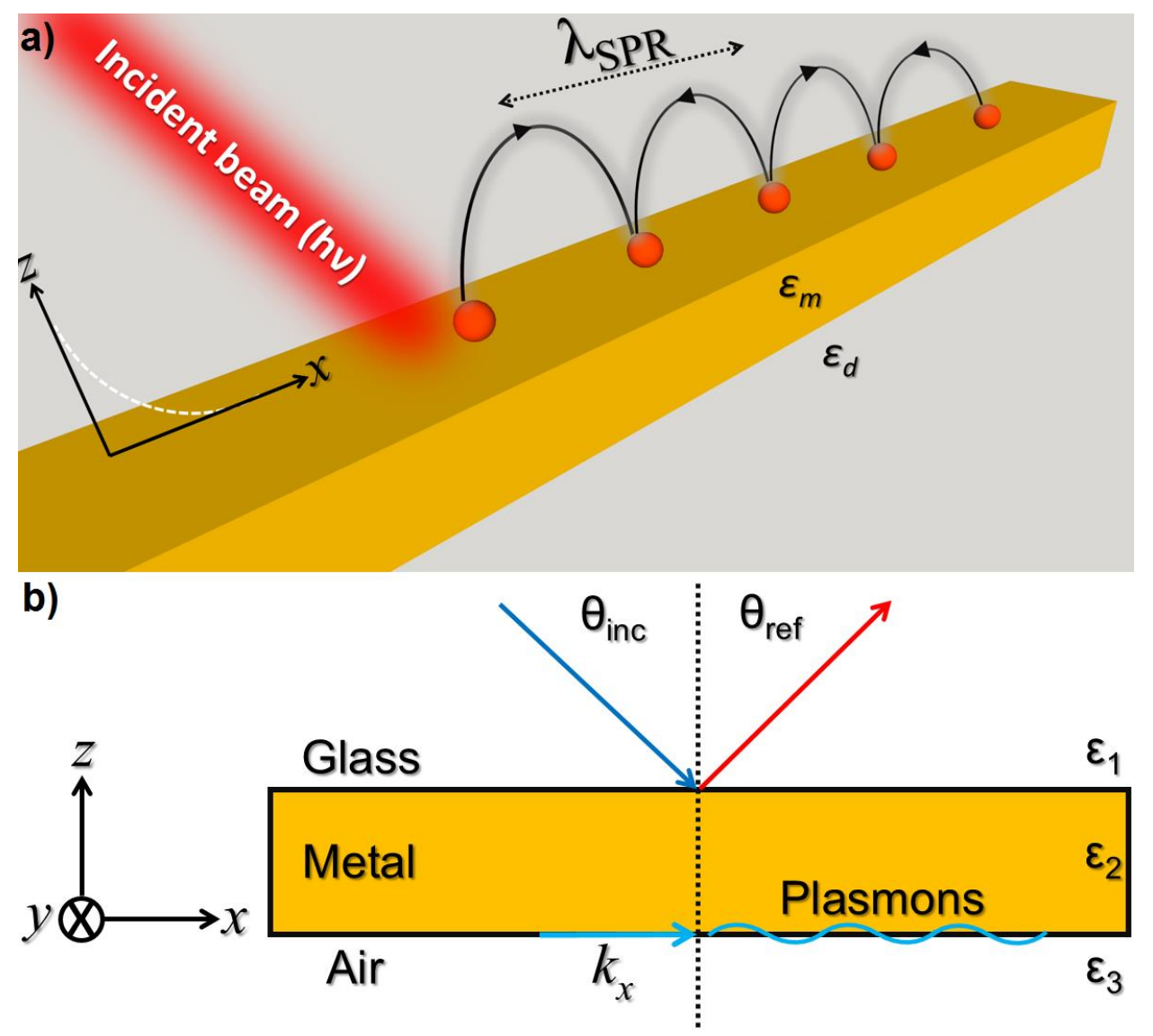

Figure 2.1. Surface plasmon resonance excitation. a) Schematic, and b) cross-sectional representations for the excitation and oscillation of plasmons at the metal-dielectric interface. 
hen a metal with negative permittivity meets another substance with a positive permittivity, therefore, an EM wave can be restricted at their interface according to the Maxwell's equations (see Fig. 2.1), as follows:

$$
\begin{aligned}
& \nabla \times \mathbf{E}=i \omega \mathbf{B} \\
& \nabla \times \mathbf{H}=-i \omega \mathbf{D} \\
& \nabla . \mathbf{E}=0 \\
& \nabla . \mathbf{H}=0
\end{aligned}
$$

The corresponding TM equations can be written as:

$$
\begin{aligned}
& \frac{\partial E_{x}}{\partial z}-\frac{\partial E_{z}}{\partial x}=i \omega \mu_{0} H_{y} \\
& -\frac{\partial H_{y}}{\partial z}=-i \omega \varepsilon_{0} \varepsilon E_{x} \\
& -\frac{\partial H_{y}}{\partial x}=-i \omega \varepsilon_{0} \varepsilon E_{z}
\end{aligned}
$$

where the corresponding TM wave equation is:

$$
\frac{\partial^{2} H_{y}}{\partial z^{2}}+\left(k_{0}^{2} \varepsilon-k_{x}^{2}\right) H_{y}=0
$$

Therefore, the electric and magnetic components above $(z>0)$ and inside $(z<0)$ the metallic surface can be written as follows:

$$
\left\{\begin{array}{l}
H_{y}=A \exp \left(-K_{1} z\right) \exp \left(i k_{x} x\right), \\
E_{x}=-\frac{A K_{1}}{i \omega \varepsilon_{0} \varepsilon} \exp \left(-K_{1} z\right) \exp \left(i k_{x} x\right), \quad(z>0) \text { where; } K_{1}=\sqrt{k_{x}^{2}-k_{0}^{2} \varepsilon_{1}} \\
E_{z}=-\frac{A k_{x}}{i \omega \varepsilon_{0} \varepsilon} \exp \left(-K_{1} z\right) \exp \left(i k_{x} x\right) .
\end{array}\right.
$$


and,

$$
\left\{\begin{array}{l}
H_{y}=B \exp \left(-K_{2} z\right) \exp \left(i k_{x} x\right), \\
E_{x}=\frac{B K_{2}}{i \omega \varepsilon_{0} \varepsilon} \exp \left(-K_{2} z\right) \exp \left(i k_{x} x\right), \quad(z<0) \text { where; } K_{2}=\sqrt{k_{x}^{2}-k_{0}^{2} \varepsilon_{2}} \\
E_{z}=-\frac{B k_{x}}{i \omega \varepsilon_{0} \varepsilon} \exp \left(-K_{2} z\right) \exp \left(i k_{x} x\right) .
\end{array}\right.
$$

Now, for the following boundary conditions:

$$
\begin{aligned}
& D_{\perp}^{1}=D_{\perp}^{2} \\
& E_{\|}^{1}=E_{\|}^{2}
\end{aligned}
$$

Then, we have:

$$
\begin{aligned}
& A=B \\
& \frac{A K_{1}}{\varepsilon_{1}}+\frac{A K_{2}}{\varepsilon_{2}}=0
\end{aligned}
$$

By considering the expressions for $K_{1}$ and $K_{2}$ as above, the dispersion relation equations are given by:

$$
\frac{k_{x}^{2} c^{2}}{\omega_{p}^{2}}=\frac{\frac{\omega^{2}}{\omega_{p}^{2}}\left(\frac{\omega^{2}}{\omega_{p}^{2}}-1\right)}{2 \frac{\omega^{2}}{\omega_{p}^{2}}-1} ;\left\{\begin{array}{l}
\varepsilon_{2}=1-\frac{\omega^{2}}{\omega_{p}^{2}} \\
k_{x}^{2}=\frac{\omega^{2}}{c^{2}}\left(\frac{\varepsilon_{1} \varepsilon_{2}}{\varepsilon_{1}+\varepsilon_{2}}\right)
\end{array}\right.
$$

Consequently, the frequency-dependent complex permittivity can be written as:

$$
\varepsilon(\omega)=1-\frac{\omega_{p}^{2}}{\omega^{2}}
$$


Also, the complex wave vector $k_{x}=k_{x}^{\prime}+i k_{x}^{\prime \prime}$ for the propagation direction can be found using the previous equations as:

$$
\left\{\begin{array}{l}
k_{x}^{\prime}=\frac{\omega}{c}\left[\frac{\varepsilon_{1}^{\prime} \varepsilon_{2}}{\varepsilon_{1}^{\prime}+\varepsilon_{2}}\right]^{1 / 2} \\
k_{x}^{\prime \prime}=\frac{\omega}{c}\left(\frac{\varepsilon_{1}^{\prime \prime}}{2\left(\varepsilon_{1}^{\prime}\right)^{2}}\right)\left[\frac{\varepsilon_{1}^{\prime} \varepsilon_{2}}{\varepsilon_{1}^{\prime}+\varepsilon_{2}}\right]^{1 / 2}
\end{array}\right.
$$

where $\varepsilon_{1}=\varepsilon_{1}^{\prime}+i \varepsilon_{1}^{\prime \prime}$ is the complex permittivity of the dielectric medium. In the other words, the complex wave vector can be written simply as follows:

$$
k_{x}=k_{x}^{\prime}+i k_{x}^{\prime \prime}=\frac{\omega}{c}\left[\frac{\varepsilon_{m} \varepsilon_{d}}{\varepsilon_{m}+\varepsilon_{d}}\right]^{1 / 2}
$$

where $\varepsilon_{m}$ and $\varepsilon_{d}$ are the permittivities of metal and dielectric media. The corresponding dispersion relation is plotted in Fig. 2.2. It should be noted that for the excitation of plasmons, it is impossible to generate such electron fluctuations directly from free space employing beam due to momentum mismatch. As shown in Fig. 2.2, the dispersion relation slightly approaches the light line at the minor complex wave vector $\left(k_{x}\right)$, which is why the excited nonradiative plasmon resonances cannot transform into light. Finally, for large complex wave vector $\left(k_{x}\right)$ and when $\varepsilon_{1}^{\prime} \rightarrow-\varepsilon_{2}$, we have:

$$
\omega_{S P R}=\sqrt{\frac{\omega_{P}}{1+\varepsilon_{2}}}
$$

In this regime, both group and phase velocities decrease significantly and approach to zero, thereby, the SPR look like a localized oscillation of electron plasma [1]. In past 

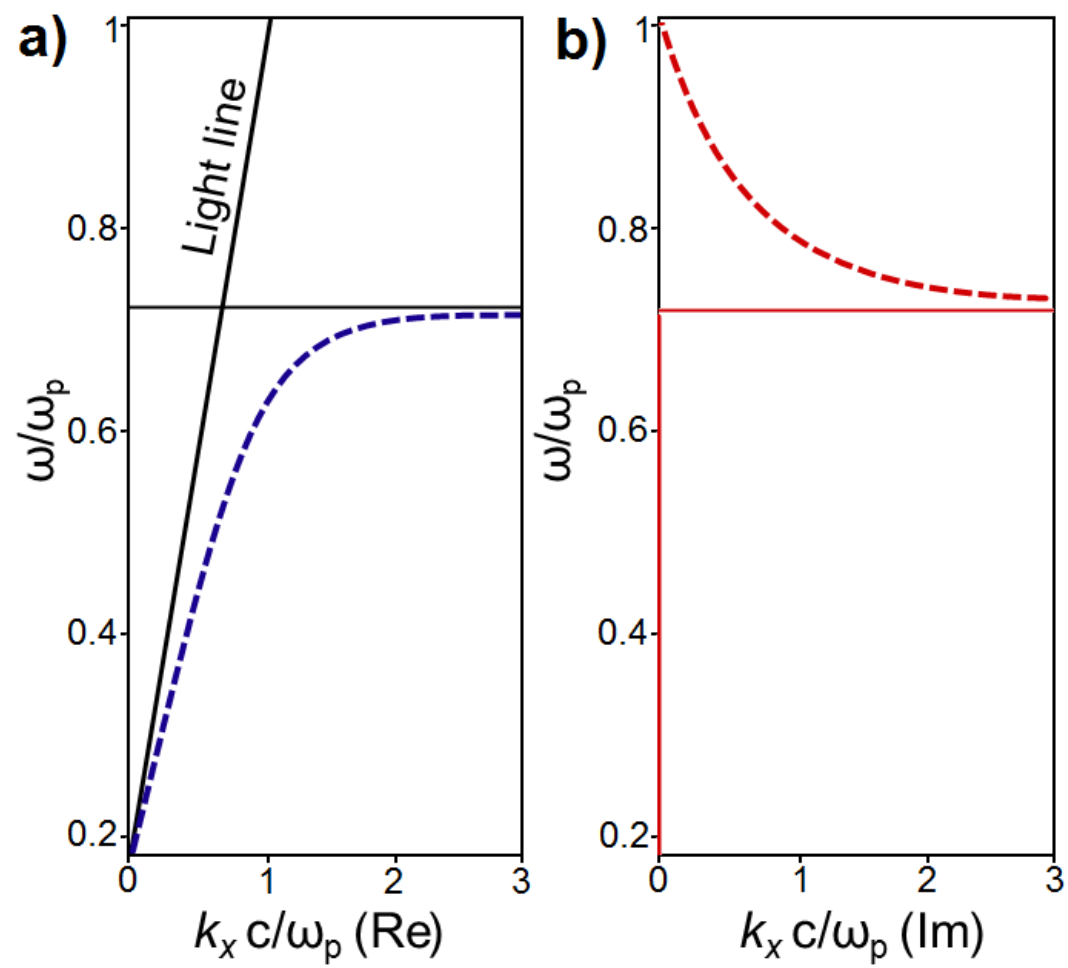

Figure 2.2. Scheme of the dispersion relation diagram of nonradiative plasmons. a) Dispersion relation as a function of real part and b) imaginary part of wave vector.

years, several methods have been carried out to excite plasmonic moments in a resonant manner based on light-matter interactions, such as Otto (see Fig. 2.3a), and Kretschmann configurations (see Fig. 2.3b). In the Otto setup a dielectric spacer between the prism (air) and the metallic surface, while in the Kretschmann configuration the dielectric prism directly contacts to the metallic surface and the dielectric section couples with the optically driven evanescent SPRs. Both setups have been broadly used for nonradiative plasmon resonance excitation and developing advanced nanophotonic systems.

\subsection{Numerical Methods}

In this subsection, we briefly describe number of well-known and promising numerical methods through analyzing their fundamental equations, which help to understand the 

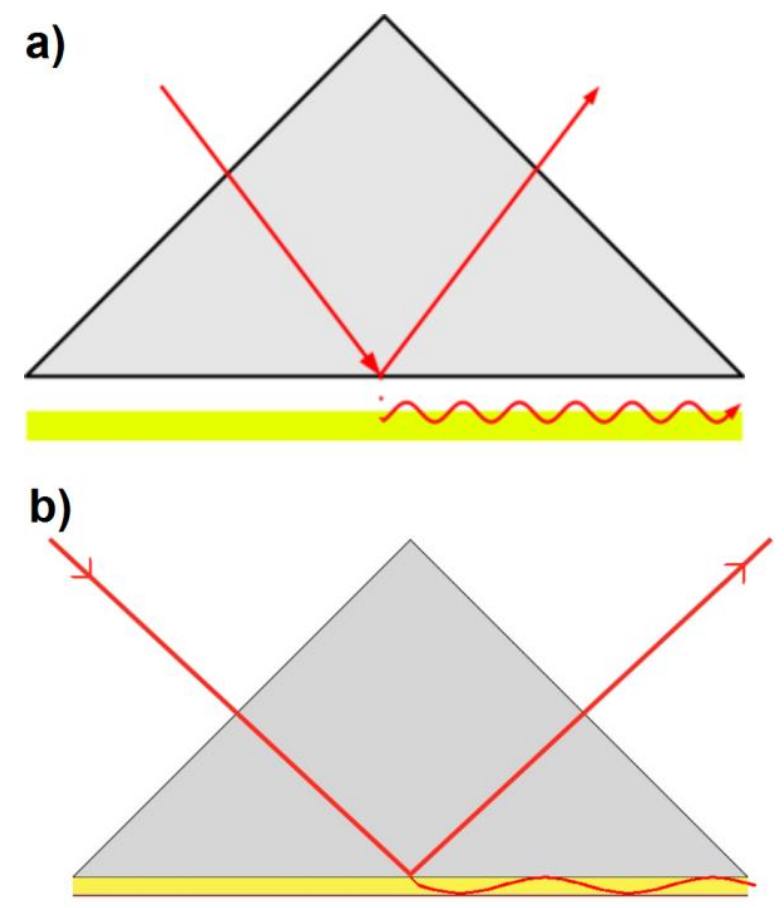

Figure 2.3. SPR excitation setups. a) Otto configuration, b) Kretschmann configuration.

type of problem correlating with subwavelength systems. Given the variety of approaches for analyzing nanophotonic and plasmonic structures, we explained some of the successful methods that are used for studying the optical response of the proposed structures and devices. One should note that most numerical methods are based on differential methods for solving the Maxwell's equations in differential form. These techniques fundamentally rely on the volume discretization in a finite computational workplace [3].

\subsubsection{FDTD Method}

The Finite-Difference Time-Domain (FDTD) algorithm is highly suited for modeling plasmon resonances and associated nanophotonic structures and nanodevices because of providing discretization flexibility and matrix free nature $[3,4]$. In this method, both time and workplace are discretized and also, all spatial and temporal derivatives in Maxwell's 
curl equations are substituted by finite difference equations [5,6]. In the FDTD framework, the most popular algorithm is introduced by Yee [7] and the corresponding details about Yee cell and additional details can be found in Refs. [3-8]. Here, we briefly explain the formulation and technical mechanism of the numerical tool. In the presence of beam source charges $(\rho)$ and currents $(\mathbf{J})$, Maxwell's equations can be written in their differential form as follows:

$$
\begin{aligned}
& \nabla \times \mathbf{H}=\frac{\partial \mathbf{D}}{\partial t}+\mathbf{J} \\
& \nabla \times \mathbf{E}=-\frac{\partial \mathbf{B}}{\partial t} \\
& \nabla . \mathbf{D}=\rho \\
& \nabla . \mathbf{B}=0
\end{aligned}
$$

In the Yee algorithm, a Cartesian grid of rectangular cells with side lengths $d_{x}, d_{y}, d_{z}$, for the spatial discretization and a time step $d t$ for the temporal discretization are selected. The time step plays fundamental role to determine the stability of numerical analysis, known as Courant stability $[9,10]$. This would help to ensure about having stable numerical results, which can be described using following relation:

$$
c_{0} d t \leq \frac{1}{\sqrt{\frac{1}{d_{x}^{2}}+\frac{1}{d_{y}^{2}}+\frac{1}{d_{z}^{2}}}}
$$

Noticing in the Maxwell's equations in Eq. 2.15, as an example, both Ampere's and Faraday's laws without the corresponding currents are considered. Taking the Ampere's law as an example, the finite-difference update equations for the electric-field in the dielectric region can be extracted as: 


$$
\begin{aligned}
E_{x_{i+1 / 2, j, k}}^{n+1}=E_{x_{i+1 / 2, j, k}}^{n} & +\frac{d t}{\varepsilon_{i+1 / 2, j, k}}\left(\frac{H_{z_{i+1 / 2, j+1 / 2, k}}^{n+1 / 2}-H_{z_{i+1 / 2, j-1 / 2, k}}^{n+1 / 2}}{d_{y}}\right) \\
& -\frac{d t}{\varepsilon_{i+1 / 2, j, k}}\left(\frac{H_{y_{i+1 / 2, j, k+1 / 2}}^{n+1 / 2}-H_{y_{i+1 / 2, j, k-1 / 2}}^{n+1 / 2}}{d_{z}}\right) \\
E_{y_{i, j+1 / 2, k}}^{n+1}=E_{y_{i, j+1 / 2, k}}^{n} & +\frac{d t}{\varepsilon_{i, j+1 / 2, k}}\left(\frac{H_{x_{i, j+1 / 2, k+1 / 2}}^{n+1 / 2}-H_{x_{i, j-1 / 2, k-1 / 2}}^{n+1 / 2}}{d_{z}}\right) \\
& -\frac{d t}{\varepsilon_{i, j+1 / 2, k}}\left(\frac{H_{z_{i+1 / 2, j+1 / 2, k}}^{n+1 / 2}-H_{z_{i-1 / 2, j+1 / 2, k}}^{n+1 / 2}}{d_{x}}\right) \\
E_{z_{i, j, k+1 / 2}}^{n+1}=E_{z_{i, j, k+1 / 2}}^{n} & +\frac{d t}{\varepsilon_{i, j, k+1 / 2}}\left(\frac{H_{y_{i+1 / 2, j, k+1 / 2}}^{n+1 / 2}-H_{y_{i-1 / 2, j, k+1 / 2}}^{n+1 / 2}}{d_{x}}\right) \\
& -\frac{d t}{\varepsilon_{i, j, k+1 / 2}}\left(\frac{H_{x_{i, j+1 / 2, k+1 / 2}}^{n+1 / 2} H_{x_{i, j-1 / 2, k+1 / 2}}^{n+1 / 2}}{d_{y}}\right)
\end{aligned}
$$

where the subscript refers to spatial grid indexing, and the superscript refers to the time step. The obtained coordinate-dependent permittivity can be effectively utilized for the dispersive models in metallic sections. In terms of accuracy, the size of the discrete cells is critical for obtaining high accuracy and small dimensions are preferred (not larger than $\lambda / 20$, where $\lambda$ is the wavelength of the incident beam). Furthermore, to reduce and minimize the spurious reflection and destructive interference of scattered waves from boundaries into the workplace, perfectly matched layers (PML) can be incorporated through a modified Nabla operator with complex stretching coordinates, where complexfrequency shifted stretching is employed [11,12]. Conventionally, in numerical methods based on the differential form of Maxwell's equations, boundary conditions must be applied to the workplace. Depending on the planned application, several types of boundaries for the surrounding field can be applied such as PML, perfectly magnetic 
conductor (PMC), metal, periodic, Bloch, Dirichlet, von Neumann, etc. It should be underlined that in the subwavelength systems with discrete symmetry, some nontrivial symmetry-driven boundary conditions can be applied on truncated domains [13]. In our analysis, the beam propagation direction is bounded with PML boundaries and the other directions are assumed to be periodic, resembles a planar metasurface composed of periodic blocks in arrays.

On the other hand, the far field can also be perfectly calculated from the numerically defined near-field spectral response without the need of extending the computation grid [5]. Using such a technique, known as near- to far-field transformation, the Green theorem is applied on surface currents that are computed at the boundaries of the computational domain. In order to obtain the far-field properties of a subwavelength system, an incident pulse can be used and the Fourier transform of the interacting field can be calculated, which yields an entire spectrum in a single calculation [5]. This methodology needs nevertheless continuing the time iteration until the field values have decayed below a given threshold value and is limited in precision for resonators with high quality factors which decay slowly with the simulation time. Ultimately, the time-domain tools such as FDTD can provide the required calculations for light propagation in an ordinary manner. Propagation lengths, both radiative and nonradiative losses can be defined in a variety of nanoscale devices. $[5,14]$. The software that is employed in this thesis for FDTD calculations is FDTD Solutions, developed by LUMERICAL Inc. [15].

\subsubsection{Finite Element Method (FEM)}

The Finite Element Method (FEM) is one of common numerical analysis tools in nanophotonics, which allows for accurate calculation of EM field in the frequency 
domain. By assuming a linear dependence of the magnetic and electric polarizations and also, by eliminating the magnetic field with the aid of the constitutive relations, the vectorial wave can be written using Maxwell's equations as [16]:

$$
\nabla \times\left(\frac{1}{\mu} \nabla \times \mathbf{E}\right)+\varepsilon \frac{\partial^{2} \mathbf{E}}{\partial t^{2}}+\sigma_{e} \frac{\partial \mathbf{E}}{\partial t}=-\frac{\partial \mathbf{J}}{\partial t}
$$

where $\mu$ is the magnetic permeability, $\varepsilon$ is the electric permittivity, and $\sigma_{e}$ is the electrical conductivity. It is noteworthy that an exponential time-dependency $\left(e^{-i \omega t}\right)$ is applied for the time-harmonic fields. In this limit, the electric field equations in the frequency domain can be written by combining the Maxwell's equations with an electrical current source $(\mathbf{J})$ as follows:

$$
\nabla \times\left(\frac{1}{\mu_{r}} \nabla \times \mathbf{E}\right)-k_{0}^{2} \varepsilon_{r} \mathbf{E}=-i k_{0} Z_{0} \mathbf{J}
$$

in which $\mu_{r}, \varepsilon_{r}, k_{0}$, and $Z_{0}$ are the relative permeability, relative permittivity, free space wave vector, and impedance, respectively. The solution of this equation with defined current and in the presence of general boundary conditions can be written as:

$$
\frac{1}{\mu_{r}} \hat{n} \times(\nabla \times \mathbf{E})+\gamma_{e} \hat{n} \times(\hat{n} \times \mathbf{E})=\mathbf{U}
$$

where $\gamma_{e}$ and $\mathbf{U}$ are known parameter and vector, respectively. Then, using the functional as below [12]: 


$$
\begin{aligned}
& F(\mathbf{E})=\frac{1}{2} \int_{V}\left(\frac{1}{\mu_{r}}(\nabla \times \mathbf{E}) \cdot(\nabla \times \mathbf{E})-k_{0}^{2} \varepsilon_{r} \mathbf{E} \cdot \mathbf{E}\right) d V \\
& +\int_{S}\left(\frac{\gamma_{e}}{2}(\hat{n} \times \mathbf{E}) \cdot(\hat{n} \times \mathbf{E})+\mathbf{E} . \mathbf{U}\right) d S-i k_{0} Z_{0} \int_{V}(\mathbf{E} . \mathbf{J}) d V
\end{aligned}
$$

Therefore, searching for the stationary point of the functional above with respect to the electric field $(\delta F=0)$ is equivalent to solving the boundary value problem including both Eqs. 2.21 and 2.22. It should be underlined that the proposed functional in Eq. 2.23 is for isotropic media and the same approach can be employed to extract a functional for an anisotropic media. In this limit, once the problem solved, the $V$ domain can be separated in a set of elements and the corresponding electric field is expanded in each element on a set of basic functions. Similar to the FDTD and other numerical computational techniques, the entire workplace must be discretized including the structure, substrate and the superstrate. Looking for stationary point of the mentioned functional in Eq. 2.23, gives rise to solution which do not satisfy the divergence condition and boundary conditions at the edges and corners. To solve these shortcomings, edge elements with vector basis functions $\mathbf{N}_{j}$ were introduced via enforcing continuity of the fields and their curl for the basis functions. This results in implicit satisfaction of Gauss' laws. For the $N$ number of unknown as the number of edges, the electric field is expanded as follows:

$$
\mathbf{E}=\sum_{j=1}^{N} E_{j} \mathbf{N}_{j}(\mathbf{r})
$$

where $E_{j}$ are the unknown coefficients. By decomposing the equation above (Eq. 2.24), the variant problem $\delta F=0$ becomes: 


$$
\sum_{j=1}^{N} M_{j k} E_{j}=b_{j}
$$

where

$$
\begin{aligned}
M_{j k}= & \int_{V} \frac{1}{\mu_{r}}\left[\left(\nabla \times \mathbf{N}_{j}\right) \cdot\left(\nabla \times \mathbf{N}_{k}\right)-k_{0}^{2} \mathbf{N}_{j} \cdot \mathbf{N}_{k}\right] d V \\
& +\int_{S} \gamma_{e}\left(\hat{n} \times \mathbf{N}_{j}\right) \cdot\left(\hat{n} \times \mathbf{N}_{k}\right) d S
\end{aligned}
$$

and

$$
b_{j}=-\int_{S} \mathbf{N}_{j} \cdot \mathbf{U} d S+i k_{0} Z_{0} \int_{V} \mathbf{N}_{j} . \mathbf{J} d V
$$

Finally, the system of equations above can be written in matrix notion consist of vector of unknown electric field intensities $(E)$, source terms $(b)$, and sparse matrix $(M)$ :

$$
M E=b
$$

The calculation of eigenvalue problems employing FEM can be also applied to lossy structures such as plasmonic waveguides and analogous nanoscale devices. It should be noted that eigenvalue equations are not specific to FEM methods but also can be found in other approaches in the frequency domain computations. Furthermore, as a differential method, the FEM method needs the use of boundaries (i.e. PML) in a similar way to other numerical programs.

Converse to FDTD method, the use of basis functions enables meshing the geometry of nanostructures with a high accuracy, which can be crucial when studying the effect of nanoscale variations of shapes on the optical properties and also sharp edges and cavities 
[17]. Such a highly fine meshing makes FEM-based techniques highly fit for the simulation of subwavelength systems based on light localization and their related applications. Dispersive materials can also be directly implemented as the FEM method is in the frequency domain. In the frequency domain, the modes as well as their resonance frequencies, losses, and area can be directly calculated, which are critical in the design of cavities for nanolasers [18]. The software that is employed in this thesis for FEM calculations COMSOL Multiphysics 5.2 modeling tool, developed by COMSOL Inc. [19].

\subsection{References}

1. Chambers, R. G. (2012). Electrons in metals and semiconductors. Thomson Press, Delhi, India.

2. Fox, M. (2010). Optical properties of solids (Vol. 2). Oxford University Press, New York.

3. Gallinet, B., Butet, J., \& Martin, O. J. (2015). Numerical methods for nanophotonics: standard problems and future challenges. Laser Photonics Reviews, 9(6), 577-603.

4. Jung, K. Y., Teixeira, F. L., \& Reano, R. M. (2007). Au/SiO $\mathrm{S}_{2}$ nanoring plasmon waveguides at optical communication band. Journal of Lightwave Technology, 25(9), 2757-2765.

5. Taflove, A., \& Hagness, S. C. (2005). Computational electrodynamics: the finitedifference time-domain method (Vol. 3). Artech House.

6. Montgomery, J. M., Lee, T. W., \& Gray, S. K. (2008). Theory and modeling of light interactions with metallic nanostructures. Journal of Physics: Condensed Matter, 20(32), 323201.

7. Yee, K. S., Chen, J. S., \& Chang, A. H. (1992). Conformal finite-different timedomain (FDTD) with overlapping grids. IEEE Transactions on Antennas and Propagation, 40(9), 1068-1075.

8. Zheng, F., Chen, Z., \& Zhang, J. (1999). A finite-difference time-domain method without the Courant stability conditions. IEEE Microwave and Guided wave letters, 9(11), 441-443. 
9. Ahmadivand, A., \& Pala, N. (2015). Localization, hybridization, and coupling of plasmon resonances in an aluminum nanomatryushka. Plasmonics, 10(4), 809-817.

10. Chew, W. C., \& Weedon, W. H. (1994). A 3D perfectly matched medium from modified Maxwell's equations with stretched coordinates. Microwave and Optical Technology Letters, 7(13), 599-604.

11. Teixeira, F. L., \& Chew, W. C. (1998). Analytical derivation of a conformal perfectly matched absorber for electromagnetic waves. Microwave and Optical Technology Letters, 17(4), 231-236.

12. Gallinet, B., Kupec, J., Witzigmann, B., \& Dupertuis, M. A. (2010). Analysis of photonic crystal defect modes by maximal symmetrization and reduction. J. Opt. Soc. Am. B, 27(7), 1364-1380.

13. Golmohammadi, S., Khalilou, Y., \& Ahmadivand, A. (2014). Plasmonics: A route to design an optical demultiplexer based on gold nanorings arrays to operate at near infrared region (NIR). Optics Communications, 321, 56-60.

14. Ahmadivand, A., Pala, N., \& Golmohammadi, S. (2015). Electromagnetic wave propagation along $\mathrm{T}$ and $\mathrm{Y}$-splitters composed of silicon nanorods, gold slots, and silica substrate. Optics Communications, 343, 73-79.

15. www.lumerical.com

16. Dhatt, G., \& Touzot, G. (2012). Finite element method. John Wiley \& Sons, NJ, USA.

17. Nauert, S., Paul A., Zhen, Y.-R., Solis Jr D., Vigderman, L., Chang, W. -S., Zubarev, E. R., Nordlander, P., \& Link, S. (2013). Influence of cross sectional geometry on surface plasmon polariton propagation in gold nanowires. ACS Nano, 8, 572-580.

18. Zhang, Q., Li, G., Liu, X., Qian, F., Li, Y., Sum, T. C., Lieber, C. M., \& Xiong, Q. (2014). A room temperature low-threshold ultraviolet plasmonic nanolaser. Nature Communications 5, 4953.

19. www.comsol.com 


\section{CHAPTER 3}

\section{Traditional Plasmon Resonant Modes}

It is well-accepted that light-matter interactions in subwavelength systems lead to the excitation of electric and magnetic dipole and multipoles with different intensities [1]. The optical properties of subwavelength materials can be determined by the corresponding EM dipole and multipole moments that can be excited in the constituent particles of a system. In this section, we present a brief explanation of the excitation and calculation of classical EM multipoles in arbitrary arrays of metallic scatterers. We also consider the excitation of dark modes in various nanoscale structures such as oligomers and multipixel resonators. This would help us to understand the formation of pronounced antisymmetric lineshapes in the system (i.e. Fano resonances). Then we present our numerical design and analysis of several novel structures and devices based on Fanoresonances for advanced photodetection and chemical sensing applications in the UV spectrum.

\subsection{Classical Electromagnetic Multipoles}

The initial result of light-matter interaction in subwavelength regime is the excitation of dipolar and multipolar moments. The classical EM multiple expansion theory has been proposed and employed to analyze the electric and magnetic fields generated by spatially localized electric charges and currents [2]. In the optical physics, the multipole expansion tool is highly appropriate for explaining the scattering of optical fields by subwavelength objects. Conventionally, if the wavelength of the incident field is large compared to the size of the object $(\lambda>>d)$, the scattering can be described by the lowest-order multipoles, the electric dipole, while the contribution of all higher-order multipoles are considered as 
pure perturbations $[2,3]$. Therefore, the geometry of the scatterer determines the types of electric current modes that can be induced in it by the incident beam. To describe these modes, a set of orthogonal electric current multipoles must be accounted, composed of elementary point currents in simple configurations. In this method, each presumed element of the induced current multipole tensor reflects the strength of one of these configurations, which leads to having the ability to visualize the real electric current modes that will be excited in the targeted scatterer.

To describe the multipole expansion of the scattered field, we firstly consider a plane wave with the electric field intensity of $E_{0}$, angular frequency of $\omega$, and wave vector of $k$. It is assumed that the particle is located in a lossless dielectric medium. The scattered EM field can be written in spherical coordinates in the form of the multipole expansion as follows [2]:

$$
\begin{aligned}
& E_{s}(r, \theta, \phi)=E_{0} \sum_{l=1}^{\infty} \sum_{m=-l}^{l} i^{l}(\pi(2 l+1))^{1 / 2} \\
& \times\left\{\frac{1}{k} a_{\mathrm{E}}(l, m) \nabla \times\left[h_{l}^{(1)}(k r) \mathbf{X}_{l m}(\theta, \phi)\right]+a_{\mathrm{M}}(l, m)\left[h_{l}^{(1)}(k r) \mathbf{X}_{l m}(\theta, \phi)\right]\right\} \\
& H_{s}(r, \theta, \phi)=\frac{E_{0}}{Z} \sum_{l=1}^{\infty} \sum_{m=-l}^{l} i^{l-1}(\pi(2 l+1))^{1 / 2} \\
& \times\left\{\frac{1}{k} a_{\mathrm{M}}(l, m) \nabla \times\left[h_{l}^{(1)}(k r) \mathbf{X}_{l m}(\theta, \phi)\right]+a_{\mathrm{E}}(l, m)\left[h_{l}^{(1)}(k r) \mathbf{X}_{l m}(\theta, \phi)\right]\right\}
\end{aligned}
$$

where $\boldsymbol{X}_{l m}$ and $h_{l}^{(1)}$ are the normalized vector spherical harmonics and the spherical Hankel functions of the first kind, respectively. The wavenumber $k$ is for the ambient with the impedance of $Z$. Here, the normalization of the multipole expansion was selected for both compact scattering and extinction cross-sections [4]. Now, by assuming the 
scattered electric field $E_{s}$ is known in the area surrounding the scatterer, we use the orthogonality properties of the vector spherical harmonics $\left(\boldsymbol{X}_{l m}\right)$ and the scalar spherical harmonics $\left(\boldsymbol{Y}_{l m}\right)$ to calculate the multipole coefficients from the distribution of the scattered electric field on any spherical surface encompassing the scatterer as:

$$
\begin{aligned}
& a_{\mathrm{E}}(l, m)=\frac{(-i)^{l+1} k r}{h_{l}^{(1)}(k r) E_{0}(\pi(2 l+1) l(l+1))^{1 / 2}} \int_{0}^{2 \pi} \int_{0}^{\pi} Y_{l m}^{*}(\theta, \phi) \hat{\mathbf{r}} \cdot \mathbf{E}_{s}(\mathbf{r}) \sin \theta d \theta d \phi \\
& a_{\mathrm{M}}(l, m)=\frac{(-i)^{l}}{h_{l}^{(1)}(k r) E_{0}(\pi(2 l+1))^{1 / 2}} \int_{0}^{2 \pi} \int_{0}^{\pi} X_{l m}^{*}(\theta, \phi) \mathbf{E}_{s}(\mathbf{r}) \sin \theta d \theta d \phi
\end{aligned}
$$

The expressions for the coefficients above can be written in terms of scattered magnetic field $\mathbf{H}_{\mathrm{s}}$ as well, as below:

$$
a_{\mathrm{M}}(l, m)=\frac{(-i)^{l} \eta k r}{h_{l}^{(1)}(k r) E_{0}(\pi(2 l+1) l(l+1))^{1 / 2}} \int_{0}^{2 \pi} \int_{0}^{\pi} Y_{l m}^{*}(\theta, \phi) \hat{\mathbf{r}} . \mathbf{H}_{s}(\mathbf{r}) \sin \theta d \theta d \phi
$$

As a specific case, for symmetric nanoparticles, all the coefficients of higher-order in $l$ than $l_{\max }$ can be made equal to zero by correctly choosing the origin of the coordinate system. The value of $l_{\max }$ strongly depends on both the size and the geometrical complexity of the nanoparticle. The assumptions and calculations above were performed for single nanoparticles. In the multiparticle systems or arrays of nanoparticles (i.e. metasurfaces), the multipole coefficients must be deduced from the distributions of the electric current density in the particles.

In continue, by applying the dielectric medium to the particle-based system, we can write the scattering current density as [3]: 


$$
\mathbf{J}_{s}(\mathbf{r})=-i \omega \varepsilon_{0}\left[\varepsilon_{r}(\mathbf{r})-\varepsilon_{r, d}\right] \mathbf{E}(\mathbf{r})
$$

Here $\varepsilon_{r, d}$ is the real-valued relative electric permittivity of the dielectric and $\varepsilon_{r}(\mathbf{r})$ is the complex-valued relative electric permittivity at any coordinate $\mathbf{r}$. The electric field in Eq. 3.5 contains both the incident and scattered fields by each nanoparticle $(j)$ in a multiparticle system:

$$
\mathbf{E}(\mathbf{r})=\mathbf{E}_{i}+\sum_{j} \mathbf{E}_{s, j}
$$

It is assumed that each discrete nanoparticle consists of a non-magnetic, isotropic, and linear material. This allows to determine a distinct scattering source current $\left(\mathbf{J}_{\mathrm{S}, j}\right)$ in each nanoparticle $(j)$ as:

$$
\mathbf{J}_{S}=\sum_{j} \mathbf{J}_{S, j}
$$

Using the Maxwell's equations, one can derive the following equations for each particle [3]:

$$
\begin{aligned}
& \nabla . \mathbf{E}_{s, j}(\mathbf{r})=-\frac{i Z}{k} \nabla \cdot \mathbf{J}_{s, j}(\mathbf{r}) \\
& \nabla . \mathbf{H}_{s, j}(\mathbf{r})=0 \\
& \nabla \times \mathbf{E}_{s, j}(\mathbf{r})=i k Z \mathbf{H}_{s, j}(\mathbf{r}) \\
& \nabla \times \mathbf{H}_{s, j}(\mathbf{r})=-\frac{i k}{Z} \mathbf{E}_{s, j}(\mathbf{r})+\mathbf{J}_{s, j}(\mathbf{r})
\end{aligned}
$$

In these series of equations, the incident EM field and the scattered field by proximal nanoparticles are discreetly present through $\mathbf{J}_{\mathrm{s}, j}$, resembling the effective current density that generates the scattered field of the $j$ th nanoparticle. Therefore, using the first and last equations in Eq. 3.8, the scalar wave equations can be obtained as [2]: 


$$
\begin{aligned}
& \left(\nabla^{2}+k^{2}\right)\left[\mathbf{r} \cdot \mathbf{E}_{s, j}(\mathbf{r})\right]=-i k Z \mathbf{r} . \mathbf{J}_{S, j}(\mathbf{r})-\frac{i Z}{k}\left(2+r \frac{d}{d r}\right)\left[\nabla . \mathbf{J}_{S, j}(\mathbf{r})\right] \\
& \left(\nabla^{2}+k^{2}\right)\left[\mathbf{r} . \mathbf{H}_{s, j}(\mathbf{r})\right]=-\mathbf{r} .\left[\nabla \times \mathbf{J}_{S, j}(\mathbf{r})\right]
\end{aligned}
$$

By solving the Eqs. 3.10 and 3.11 and inserting them into Eqs. 3.3 and 3.4, one can obtain the multipole coefficients as below [3]:

$$
\begin{aligned}
& a_{\mathrm{E}}(l, m)=\frac{(-i)^{l-1} k Z}{E_{0}(\pi(2 l+1) l(l+1))^{1 / 2}} \int Y_{l m}^{*}(\theta, \phi) j_{l}(k r)\left\{k^{2} \mathbf{r} . \mathbf{J}_{S, j}(\mathbf{r})+\left(2+r \frac{d}{d r}\right)\left[\nabla \cdot \mathbf{J}_{S, j}(\mathbf{r})\right]\right\} d^{3} r(3 . \\
& a_{\mathrm{M}}(l, m)=\frac{(-i)^{l-1} k^{2} Z}{E_{0}(\pi(2 l+1) l(l+1))^{1 / 2}} \int Y_{l m}^{*}(\theta, \phi) j_{l}(k r) \mathbf{r} \cdot\left[\nabla \times \mathbf{J}_{S, j}(\mathbf{r})\right] d^{3} r
\end{aligned}
$$

where $j_{l}$ is the spherical Bessel functions. Using the integration of Eqs. 3.12 and 3.13 by parts to cast in the form below [3]:

$$
\begin{aligned}
a_{\mathrm{E}}(l, m)= & \frac{(-i)^{l-1} k^{2} Z O_{l m}}{E_{0}(\pi(2 l+1))^{1 / 2}} \int \exp (-i m \phi)\left\{\left[\Psi_{l}(k r)+\Psi_{l}^{\prime \prime}(k r)\right] P_{l}^{m}(\cos \theta) \widehat{\mathbf{r}}_{.} \mathbf{J}_{S, j}(\mathbf{r})\right. \\
& \left.+\frac{\Psi_{l}^{\prime}(k r)}{k r}\left[\tau_{l m}(\theta) \hat{\theta} . \mathbf{J}_{S, j}(\mathbf{r})-i \pi_{l m}(\theta) \widehat{\phi} . \mathbf{J}_{S, j}(\mathbf{r})\right]\right\} d^{3} r
\end{aligned}
$$

$$
\begin{aligned}
a_{\mathrm{M}}(l, m) & =\frac{(-i)^{l+1} k^{2} Z O_{l m}}{E_{0}(\pi(2 l+1))^{1 / 2}} \\
& \times \int \exp (-i m \phi) j_{l}(k r)\left[i \pi_{l m}(\theta) \hat{\theta} . \mathbf{J}_{S, j}(\mathbf{r})+\tau_{l m}(\theta) \widehat{\phi} . \mathbf{J}_{S, j}(\mathbf{r})\right] d^{3} r
\end{aligned}
$$


where $\Psi_{l}(k r)=k r j_{l}(k r)$ are the Riccati-Bessel functions, and the parameters $\Psi_{l}^{\prime}(k r)$ and $\Psi_{l}^{\prime \prime}(k r)$ are the first and second derivatives with respect to the argument $k r$. The corresponding Legendre polynomials are discussed by Jackson [2]. In additions the following functions are employed in the equations above:

$$
\begin{gathered}
O_{l m}=\frac{1}{[l(l+1)]^{1 / 2}}\left(\frac{2 l+1}{4 \pi} \frac{(l-m) !}{(l+m) !}\right)^{1 / 2} \\
\tau_{l m}(\theta)=\frac{d}{d \theta} P_{l}^{m}(\cos \theta) \\
\pi_{l m}(\theta)=\frac{m}{\sin \theta} P_{l}^{m}(\cos \theta)
\end{gathered}
$$

Ultimately, for the scattering of light by a single nanoparticle, we can introduce the scattering cross-sectional spectra based on multipole coefficients based on Bohren method [5]:

$$
C_{s}=\frac{\pi}{k^{2}} \sum_{l=1}^{\infty} \sum_{m=-l}^{l}(2 l+1)\left[\left|a_{\mathrm{E}}(l, m)\right|^{2}+\left|a_{\mathrm{M}}(l, m)\right|^{2}\right]
$$

and the extinction cross-section can be defined as below by following the same methodology, where the incident beam is $x$-polarized:

$$
C_{e x t}=-\frac{\pi}{k^{2}} \sum_{l=1}^{\infty} \sum_{m=-l,+1}(2 l+1) \operatorname{Re}\left[m a_{\mathrm{E}}(l, m)+a_{\mathrm{M}}(l, m)\right]
$$


For $y$-polarized beam, the extinction cross-section can be written as:

$$
C_{e x t}=\frac{\pi}{k^{2}} \sum_{l=1}^{\infty} \sum_{m=-l,+1}(2 l+1) \operatorname{Im}\left[a_{\mathrm{E}}(l, m)+m a_{\mathrm{M}}(l, m)\right]
$$

Excitation of classical EM modes is not the sole result of light-matter interaction in nanoscale dimensions. In closely-packed and multiparticle systems such as nanoparticles clusters, excitation of various types of moments is possible that will be discussed in the following sections.

\subsection{Hybridization Theory}

Ultrastrong confinement and interaction of optically driven plasmons in subnanometer spots lead to hybridization of plasmons, has been employed as a theoretical approach to describe the sensitive structural tunability of the plasmon resonance frequency of any arbitrary shape of nanoparticles and also employed for developing advanced nanophotonic and bio-related devices [6-16]. The first observation of the plasmon resonance hybridization has been reported in concentric nanoshells or nanomatryoshka arrangement with high geometrical tunability and the ability to strongly confine plasmons (see Fig. 3.1). According to the hybridization theory in metallic nanoscale components, the electron gas deformations can be decomposed as spherical harmonics of order $n$. In this regime, the interaction of the excited plasmons on the inner and outer surfaces of a given nanoshell leads to two hybridized plasmonic modes for each positive harmonic order. Thereby, the frequencies of these moments can be described as [15]: 


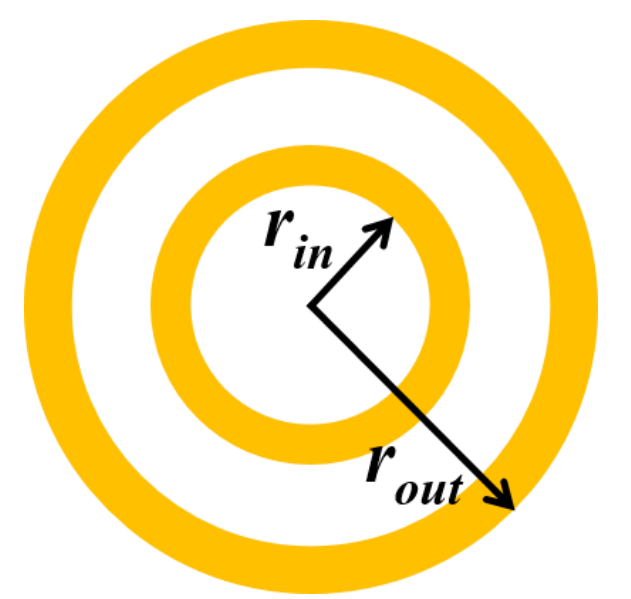

Figure 3.1. A representation of plasmonic nanomatryoshka.

$$
\omega_{n \pm}^{2}=\frac{\omega_{B}^{2}}{2}\left(1 \pm \frac{1}{2 n+1} \sqrt{1+4 n(n+1)\left(\frac{r_{\text {in }}}{r_{\text {out }}}\right)^{2 n+1}}\right)
$$

where the $\left|\omega_{+}\right\rangle$mode corresponds to antisymmetric coupling between the sphere and cavity modes and the $\left|\omega_{-}\right\rangle$mode corresponds to symmetric coupling between the two modes (see Fig. 3.2). Figure 3.2 illustrates a schematic model for the plasmon resonance hybridization for a nanomatryoshka system. Such an expression for the nanoshell plasmon resonance energies has been confirmed using quantum mechanical computations [17]. Although there have been outstanding progresses on the excitation of both spherical and nonspherical nanoparticles, the oligomers family consisting of spherical nanoparticles have witnessed growing attention because of possessing exquisite spectral lineshapes in hybridized plasmons states [18]. The simplest member of oligomers family is a pair of adjacent nanoparticles, known as dimer antenna, depicted in Fig. 3.3 [16]. Simple dimer aggregates allows for better understanding of plasmon hybridization concept. 
In the plasmon hybridization theory, the conduction band electrons are modeled as charged, incompressible liquid locating on top of a rigid, positive charges represents the ion cores [19]. According to Nordlander et al. [19], the ion cores are treated within the

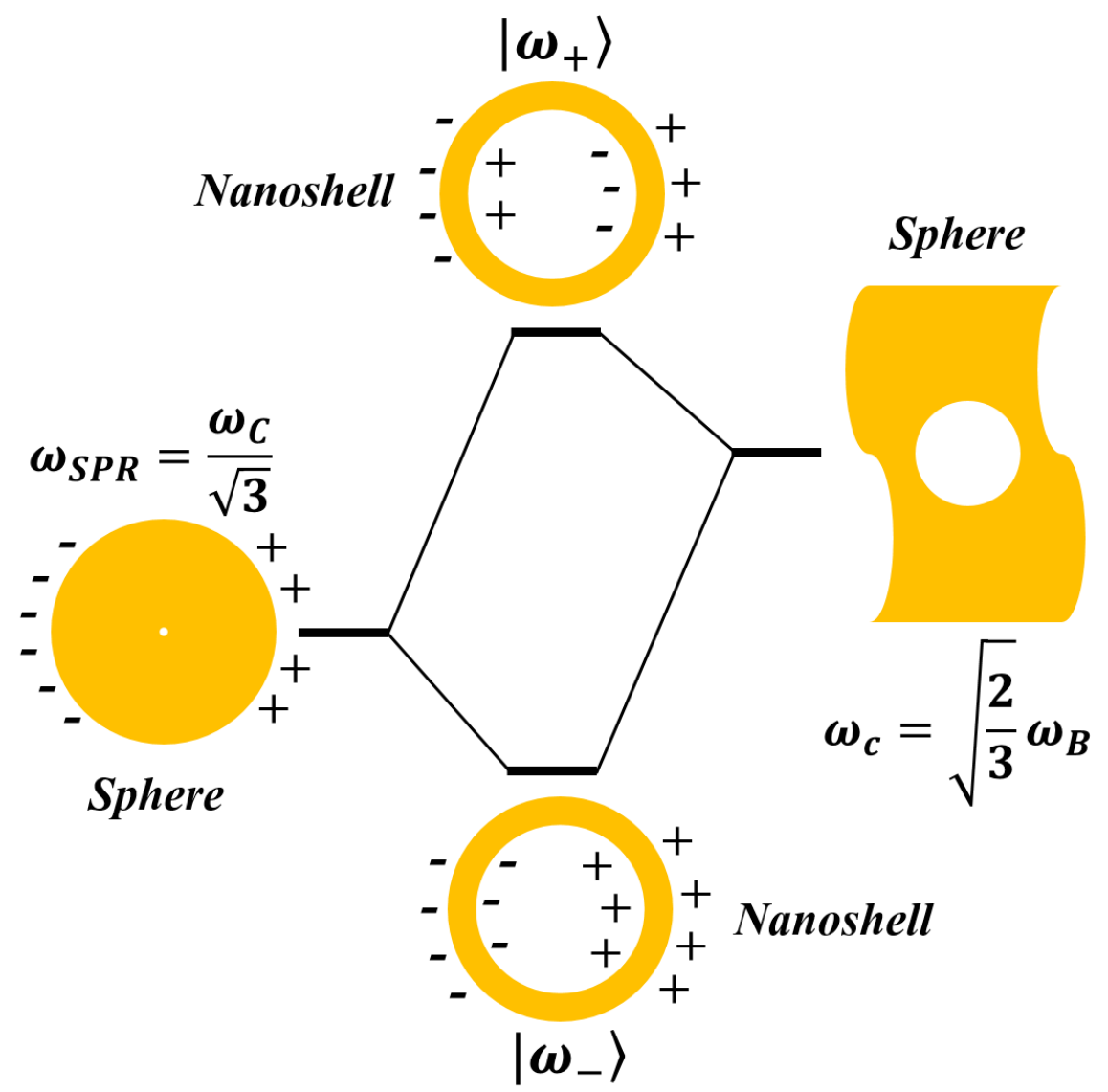

Figure 3.2. An energy level diagram, for the hybridization model in metallic nanoshells resulting interaction between the sphere and cavity plasmons.

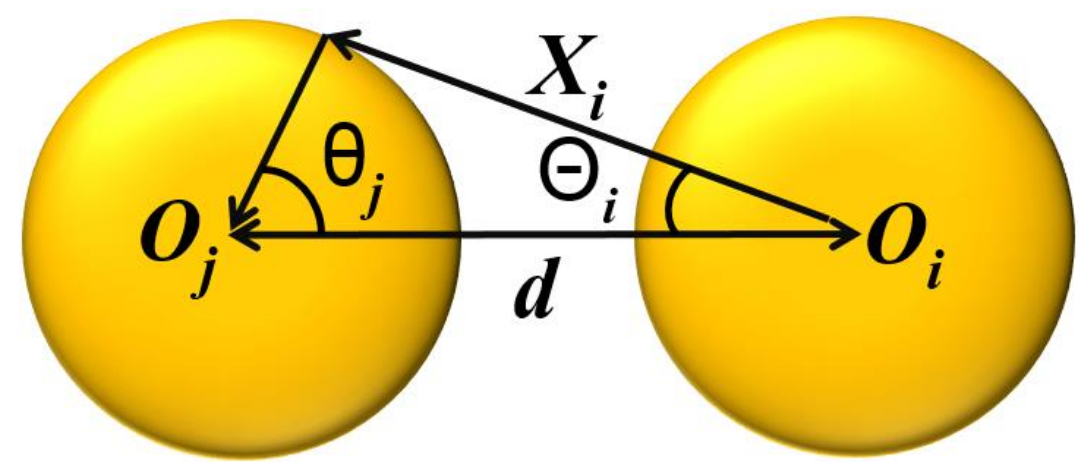

Figure 3.3. Schematic art picture of a dimer antenna in hybridized condition 
jellium approximation, hence, the positive charge $\left(n_{0}\right)$ is consistently allocates within the nanoparticle's boundaries. Being consistent with both classical calculations, $a b$ initio computations, and generalized multiparticle Mie theory [20], the proposed hydrodynamic method gives rise to the plasmon energies for metallic nanoparticles larger than a few nanometers. The plasmonic resonant modes can be resembled as self-sustained deformations of electron incompressible liquid. Therefore, the mere effect of such deformations is the formation of surface charges. These surface charges for an individual metallic nanosphere can be written by [9]:

$$
\sigma(\Omega, t)=n_{0} e \sum_{l, m} \sqrt{\frac{l}{R^{3}}} S_{l m}(t) Y_{l m}(\Omega)
$$

where $R$ is the radius of spherical nanoparticle, $Y_{l m}(\Omega)$ is a spherical harmonic of the solid angle $\Omega$, and $S_{l m}$ is the deformations amplitude defined the new degrees of freedom.

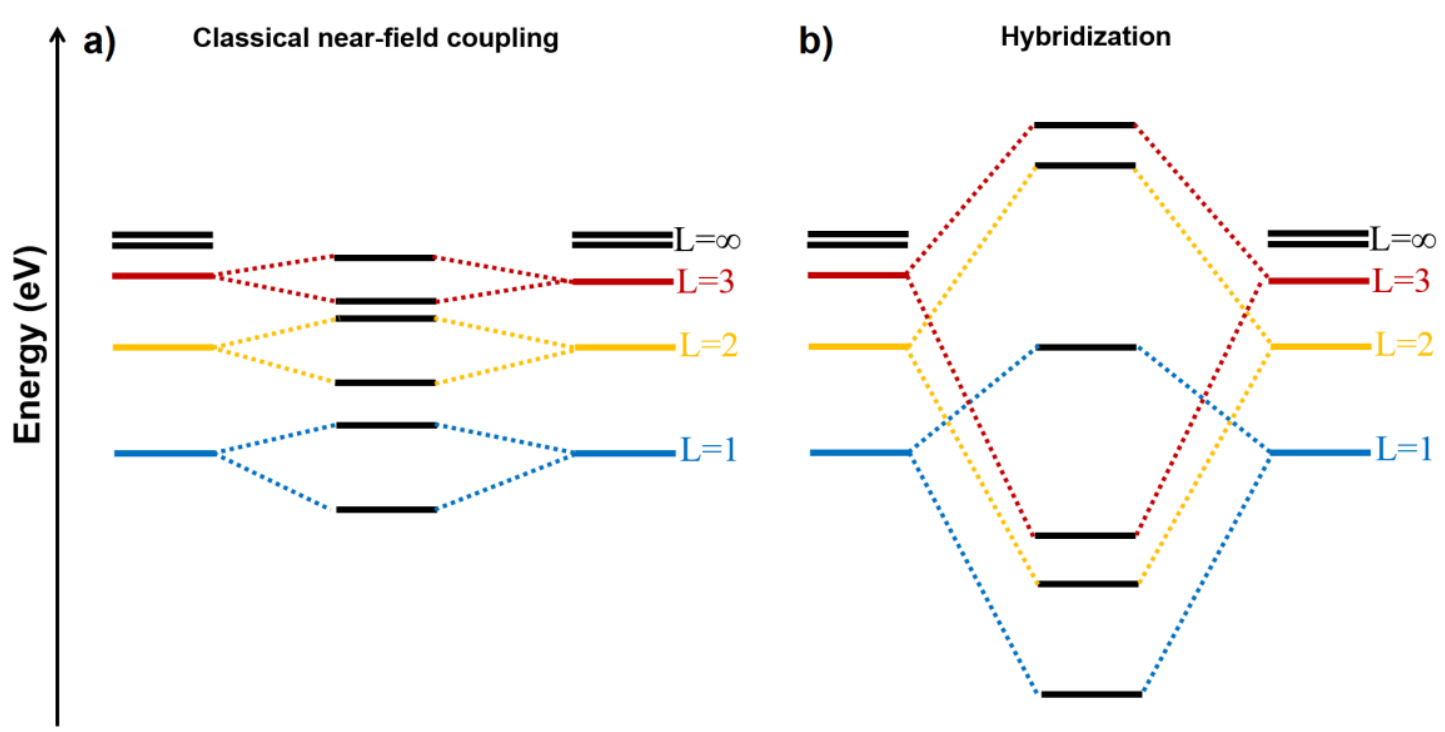

Figure 3.4. Energy level diagram for plasmon resonances coupling in a) classical nearfield and b) hybridized regimes for a dimer nanostructure. 
Accordingly the dynamics of the deformations is described by following Lagrangian:

$$
L_{S}=\frac{n_{0} m_{e}}{2} \sum_{l, m}\left(\dot{S}_{l m}^{2}-\left[\omega_{B} \sqrt{\frac{l}{2 l+1}}\right] S_{l m}^{2}\right)
$$

where the bulk plasmon frequency is:

$$
\omega_{B}=\sqrt{\frac{4 \pi e^{2} n_{0}}{m_{e}}}
$$

For a pair of spherical nanoparticles (with a separation distance of $D$ ) with subwavelength dimensions, the retardation effect can be simply neglected. Therefore, the dynamic of the plasmons can be estimated and defined by the instantaneous Coulomb interaction between the surface charges as below as a function of intercenter distance between nanoparticles:

$$
V(D)=\int R_{1}^{2} d \Omega_{1} \int R_{2}^{2} d \Omega_{2} \frac{\sigma^{1}\left(\Omega_{1}\right) \sigma^{2}\left(\Omega_{2}\right)}{\left|\vec{r}_{1}-\vec{r}_{2}\right|}
$$




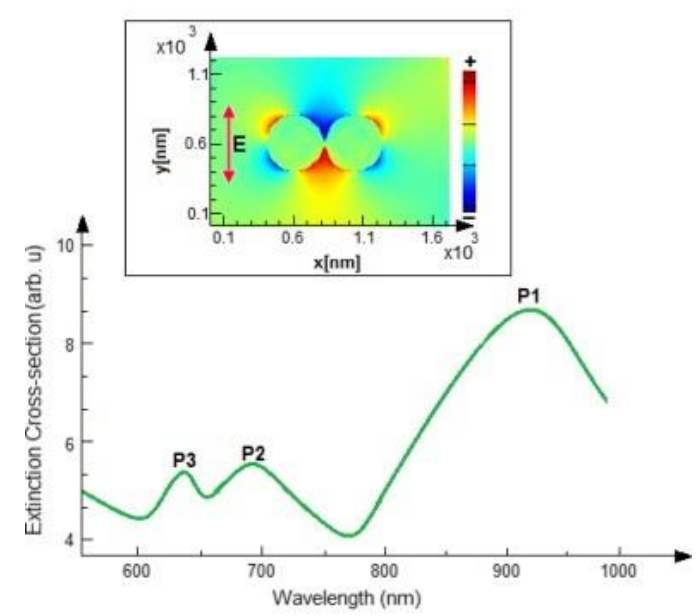

Figure 3.5. Plasmonic response of an Al nanodimer. The extinction spectra and the E-field map are calculated numerically. The resonant multipolar peaks are denoted by $\mathbf{P}$.

el can be optimized for the polar axis along the dimer assembly axis and a real representation can be applied for the spherical harmonics [16]. As a specific case, dimer assembly is strongly polarization-dependent and for the excitation polarization along the dimer axis, large plasmonic resonance shifts in hybridized regime can be expected [1,21]. Conversely, for the transverse beam excitation, the structure does not show significant variations in the plasmonic response. In terms of plasmon hybridization theory, while the proposed dimer is hybridized, the bonding diagram reflects strong perturbations in the energy levels of bonding modes. Figure 3.4a exhibits the energy level diagram for a dimer assembly consisting of gold nanoparticles with the radii of $60 \mathrm{~nm}$, located apart with two gap openings. For the dimer antenna with distant nanoparticles from each other, the arising moments weakly couple to each other. While for the proximal nanoparticles with a few nanometers gap spot between nanodisks, the induced moments strongly interact and leads to severe perturbations in the energy band diagram $[21,22]$ as shown in Fig. 3.4b. 
Figure 3.5 illustrates the hybridization of plasmons for a dimer structure composed of Al nanorings in strongly hybridized regime, obtained by our numerical analysis based on FDTD method. As a strategic and alternative metal for conventional costly noble metals for plasmonic purposes, Al has received growing attention in recent years due to providing low-cots nanofabrication feature and absence of interband transitions in the UV to the visible domain [23]. Considering the fast that $\mathrm{Al}$ suffers from rapid oxidation at low energy levels, the plotted profile in Fig. 3.5 is obtained by applying a few nanometer of oxide coverage around the nanoparticles $\left(\mathrm{Al}_{2} \mathrm{O}_{3}\right)$. To this end, the Bruggeman model was applied to model the natural composition of $\mathrm{Al} / \mathrm{Al}_{2} \mathrm{O}_{3}$ dielectric function using the equation below [24]:

$$
n_{A l}\left[\frac{\varepsilon_{A l}-\varepsilon}{\varepsilon_{A l}+2 \varepsilon}\right]+n_{A l}\left[\frac{\varepsilon_{o x}-\varepsilon}{\varepsilon_{o x}+2 \varepsilon}\right]=0
$$

where $n_{A l}$ and $n_{o x}$ are the volume functions of $\mathrm{Al}$ and oxide encompassing the material, respectively. Here, to characterize the plasmonic response of the structure, we used the modified Drude model as:

$$
\varepsilon_{A l}=\varepsilon_{\infty}-\frac{\omega_{p}^{2}}{\omega(\omega+i \Gamma)}
$$

where $\omega_{p}$ is the bulk plasmon frequency, $\Gamma$ is the damping constant, and $\varepsilon_{\infty}$ is the highfrequency response. According to empirically defined values, following settings were applied to extract the spectral response of the structure: The thickness of the oxide cover is assumed to be $4 \mathrm{~nm}$, with metallic Drude damping of $\Gamma=1.2 \mathrm{eV}$ and $\varepsilon_{\infty}=3$. Under the 
plane wave illumination, three multipolar resonant modes are excited along the UV to the NIR spectra correlating with the dipolar $\left(\mathrm{P}_{1}\right)$, quadrupolar $\left(\mathrm{P}_{2}\right)$, and octupolar $\left(\mathrm{P}_{3}\right)$ resonant moments. The inset is the E-field map, showing the resonance excitation and near-field coupling at the gap between proximal nanoparticles.

The provided method works properly and accurately for simple nanoparticle assemblies (i.e. dimer, trimer), however, this model becomes extremely complicated for more complex nanostructure and nanoparticle assemblies. Therefore, numerical tools and programs can be considered as promising and simple approaches to study and estimate the plasmonic response of colloidal and self-assembled plasmonic nanoassemblies.

\subsubsection{Dark Modes}

As we mentioned in prior section, plasmonic dark modes are pure near-field resonances that can be successfully induced through the hybridization of plasmonic momenta interferences in a set of closely-packed nanoparticles in a quasi-static approximation [6]. In addition, dark modes also arise from the interaction of the bright modes of strongly coupled nanoparticles. Possessing longer lifetime compared to the bright modes, absence of classical losses, and lack of net dipole moments made the dark modes attractive for several applications from sensing [6] to waveguiding $[13,14]$. As it is presented in the earlier section, the simplest example of the excitation of dark modes is a coupled nanodimer composed of two adjacent nanoparticles, in which a dark mode can originate from an out-of-phase oscillation of the bonding superradiant bright mode (or dipolar moment) of the particles. This resulting in excitation of collective plasmon mode without a net dipole moment. Dark plasmonic modes cannot be directly excited with linearly 
polarized beams, and in turn, one needs other illumination techniques and the proper geometries to excite such modes. As the initial and basic components, geometrical variations and symmetry breaking have been employed as the fundamental methods to excite dark plasmons in nanoparticle-based structures and antennas based on blocks $[11,25,26]$. Besides, several approaches have been carried out to excite dark plasmons in both symmetric and antisymmetric structures based on metallic and all-dielectric nanoparticles, such as using cylindrical vector beams (radially and azimuthally polarized lights), vortex beam to excite dark plasmons, and focused electron beams [6,27-30]. This was accomplished by using tailored far-field radiations techniques such as spatially inhomogeneous fields [31], evanescent excitation [32], subtle retardation effects [33], and spatial phase reshaping [34].

In terms of the optical physics, the interaction between LSPR excites in their electric near-fields can be resulted in Coulomb-type interaction between dipoles. Electrostatic approximation has been employed to model these interactions by neglecting the retardation effects on the basis of the subwavelength dimensions of nanoparticles [6,35]. This model indicates that the coupling between two neighbor particles (A and B) can be described by a coupling coefficient as follows [36]:

$$
C_{A B}=-\frac{G_{A B}}{\omega-\omega_{A}+i \frac{\Gamma}{2}}
$$

where $G_{A B}$ is a factor defining the geometrical coupling between the particles, $\omega$ is the applied field frequency, and $\omega_{A}-i \Gamma / 2$ is the complex resonance frequency of confined 
plasmons. Here, the geometrical factor $\left(G_{A B}\right)$ can be described as a dipole-dipole coupling term as [36]:

$$
G_{A B} \propto \frac{\left[3\left(\vec{p}_{A} \cdot \vec{d}\right)\left(\vec{p}_{B} \cdot \vec{d}\right)-\left(\vec{p}_{A} \cdot \vec{p}_{B}\right)\right]}{d^{3}}
$$

where $d$ is the intercenter distance between nanoparticles and $\vec{p}_{A B}$ are the dipole moments, and $\vec{d}$ is a unit vector along the line separating the nanoparticles. For smaller openings between nanoparticles, the high-order multipolar resonant moments can be excited and must be included in the expansion of the geometrical factor, hence, the coupling coefficient can be written as below, which includes all multipolar terms $l$ and $j$ of each individual particles:

$$
C_{A B} \propto \sum_{l, j} C_{A B}^{l, j}
$$

In this limit, for an isolated nanoparticle (A), the incident beam will excite plasmon resonances at the surface of nanoparticle with the charge distribution of $\sigma(\vec{r})$. Such a charge distribution can be written in terms of the eigenmodes of the nanoparticle $\left(\sigma_{A}^{m}(\vec{r})\right.$ ) according to [6]:

$$
\sigma(\vec{r})=\sum_{m} a_{A}^{m}(\omega) \sigma_{A}^{m}(\vec{r})
$$

where $a_{A}^{m}(\omega)$ are the excitation amplitudes, describes the coupling of the incident EM wave field with a particular eigenmode, and $m$ is an index which indicates the $m$ th mode 


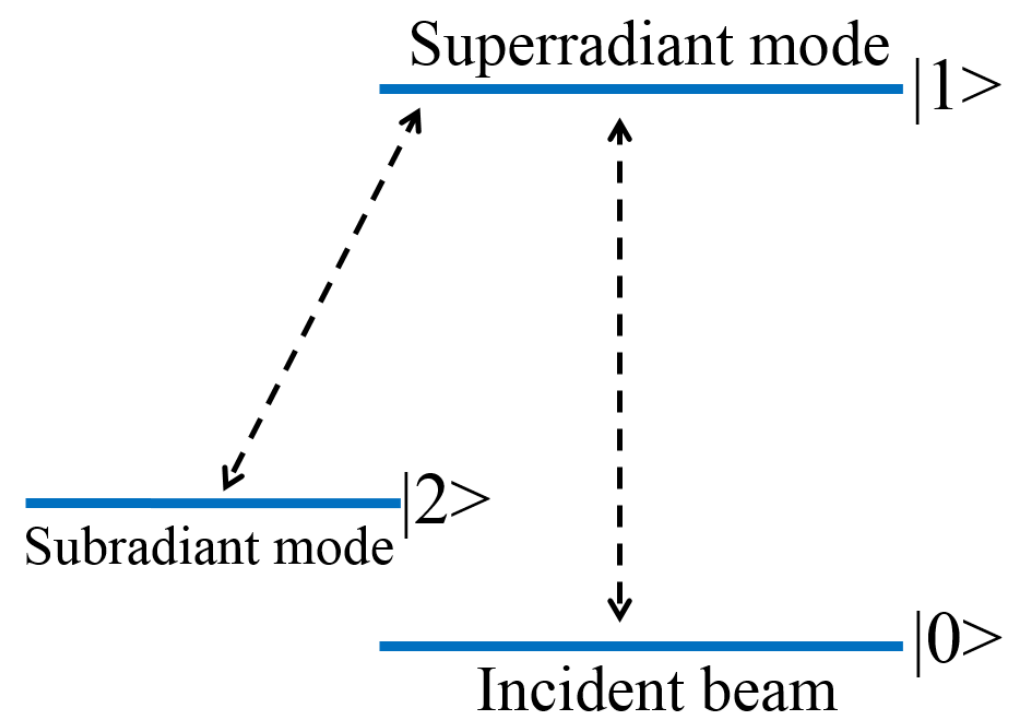

Figure 3.6. Schematic of interference between the superradiant and subradiant modes in a unit cell.

sustained by particle A. The arisen modes in this regime consist of doubly degenerate superradiant binding modes which are spanning the irreducible representation, including substantial red-shift in the corresponding frequency of plasmonic modes from proximal nanoparticles. The excited dipole moment in this regime can be written as:

$$
\vec{p} \propto \frac{\cos (\varphi) \hat{x}+\sin (\varphi) \hat{y}}{G+\left(\omega-\omega_{A}+i \frac{\Gamma}{2}\right)}
$$

in which $\varphi$ is the angle of incident linearly polarized beam, and $G$ is the interparticle coupling factor. The induced eigenmodes of the system strongly depends on the symmetry of the structure and dark mode between them can be identified by its narrowness, blue-shift and zero dipole moment. 


\subsubsection{Fano Resonances}

The spectral response of a certain plasmonic subwavelength structure determines by the corresponding behavior of the excited surface plasmon resonances. In nanoparticlescoupled systems, strong field enhancement between closely-packed nanoparticles gives rise to formation of energetic hotspots. Depending on the morphological and inherent characteristics of these components, some of them display unique spectral lineshapes known as Fano resonances, characterized by a narrow spectral transparency windows. In the Fano minimum wavelength, the scattering significantly suppresses and the absorption cross-section enhances $[11,12,23,26-28,37,38]$. It is well-acknowledged that Fano resonances arise from weak and destructive interaction between subradiant dark and superradiant bright modes in the energy continuum of the bright mode. Figure 3.6 demonstrates the classical schematic of interference between the superradiant and subradiant moments in a given unit cell. It should be underlined that in the retarded region, the dark mode cannot couple directly to the incident beam. Furthermore, in terms of Mie theory for light scattering principle [2], the fundamental components of extinction cross-section are the scattering and absorption spectra, given by the following expression: $\sigma=\pi d^{2} Q$, where $d$ is the size of the nanoparticle or nano-object, and $Q$ is the corresponding efficiency. Therefore, the scattering cross-section can be written as a function of resonance wavelength $(\lambda)$, geometrical parameters, and EM intensities $\left(a_{\lambda}\right.$ and $b_{\lambda}$ ) as (the simplified form of Eq. 3.20):

$$
Q_{\text {ext }}=\frac{2}{q^{2}} \sum_{\lambda=1}^{\infty}(2 \lambda+1)\left[\left|a_{\lambda}\right|^{2}+\left|b_{\lambda}\right|^{2}\right]
$$




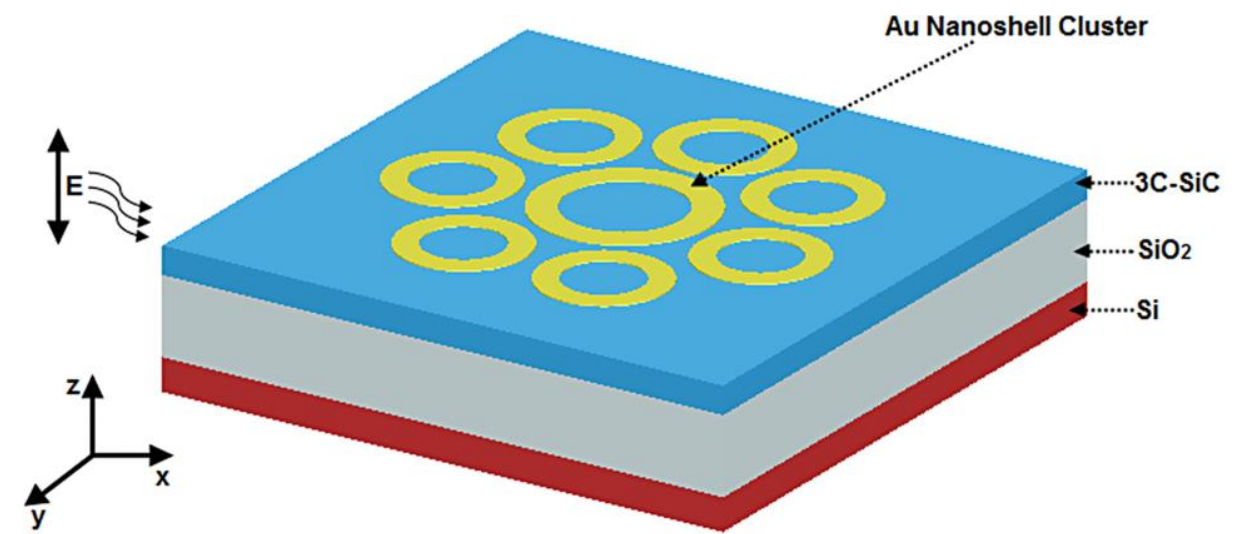

(a)

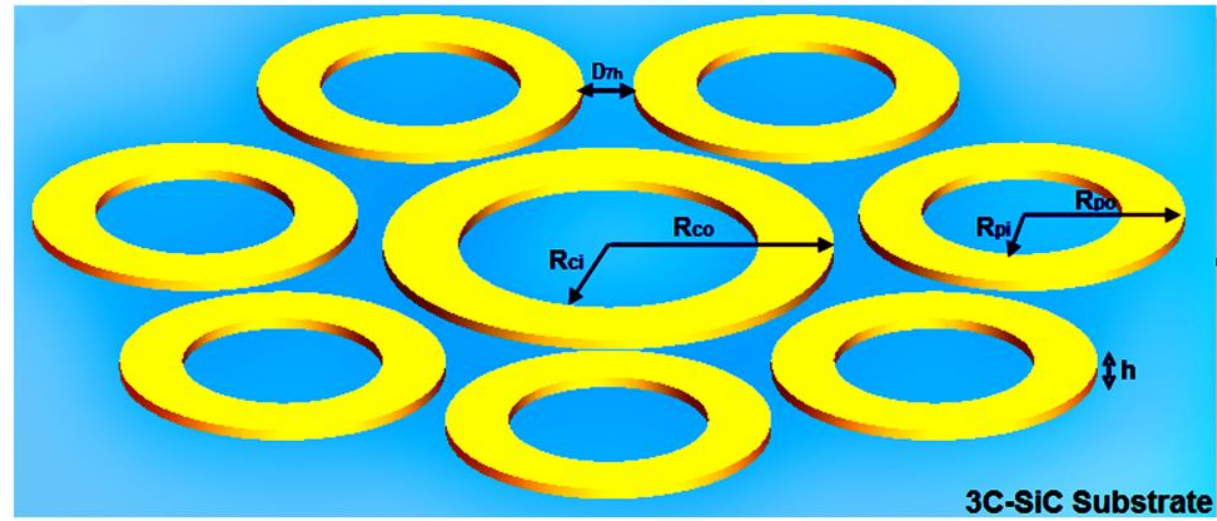

(b)

Figure 3.7. (a) Schematic and (b) top-view image of an eight-member octamer cluster composed of gold nanorings on a multilayer substrate.

size parameter, defining by $q=\omega d / c$, where $c$ is the velocity of light in a vacuum, and $\omega$ is the incident-beam frequency. The scattering intensities are reported by Bohren [5]. In the radiative coupling limit [38], an interaction between the dipolar (as the bonding bright mode) and quadrupole (as the antibonding dark mode) resonances leads to the excitation of Fano resonant moment. Interference of the incident and re-emitted beam generates a complex near-field pattern and gives rise to both destructive (field suppression) and constructive (field enhancement) interference of the EM field, similar to the Fano resonances arisen from quantum particles scattered by a potential with quasidiscrete 
levels [39]. Depend on the geometry of a given nanostructure, high multipolar surface modes can also interfere with the broad dipole moment and generate higher-order Fano resonances when the size of the system increased. The simplest example of the double or triple Fano-resonant nanostructures is the symmetric heptamer composed of split-rings, proposed by Zhang et al. [40].

In the next section, we present our numerical and analytical studies for the excitation of Fano lineshapes in plasmonic assemblies for biochemical and gas sensing as part of this dissertation work.

\subsubsection{Fano-Resonant Asymmetric Nanoring Clusters}

As an example for the formation of Fano lineshape, Figs. 3.7a and 3.7b illustrate the schematics for a plasmonic octamer cluster composed of gold nanorings, closely coupled to each other with the description to the geometrical parameters [10]. It is assumed that the entire cluster is deposited on a multilayer substrate consisting of $\mathrm{Si}, \mathrm{SiO}_{2}$, and $3 \mathrm{C}-\mathrm{SiC}$ sublayers to provide strong confinement of light and induce strong Fano lineshape. The refractive index of each layer was set to: $n_{\mathrm{Si}}=2.647, n_{\mathrm{SiO} 2}=1.446$, and $n_{\mathrm{SiC}}=3.497$ [41]. Employing Au substance with the empirically defined refractive indices by JohnsonChristy measurements [42], we calculated the plasmonic response of the structures and the formation of Fano resonant dip numerically, shown in Fig. 3.8. The utilized geometrical sizes are listed inside the picture and the thickness and gap distance between nanorings are fixed to $60 \mathrm{~nm}$ and $12.5 \mathrm{~nm}$. Technically, the two relevant modes for Fano interference are a superradiant bright mode, where the dipolar plasmons of all nanorings oscillate in-phase and in the same direction. In addition, a subradiant dark mode for the middle nanoparticle oscillate out-of-phase respect to the surrounding nanorings. The 
Fano dip here arises in the retarded limit and in this regime, the bright mode becomes superradiant and the dark mode remains subradiant. In this limit, a weak and destructiuve interference between the dark and bright modes gives rise to the formation of a minimum in the scattering spectra. Here, we observed the excitation of distinct Fano lineshape around the near infrared region for variations in both inner and outer radii of nanorings homogenously. For the inner radius variations $\left(R_{c i}\right)$, increasing the inner radius red-shifts

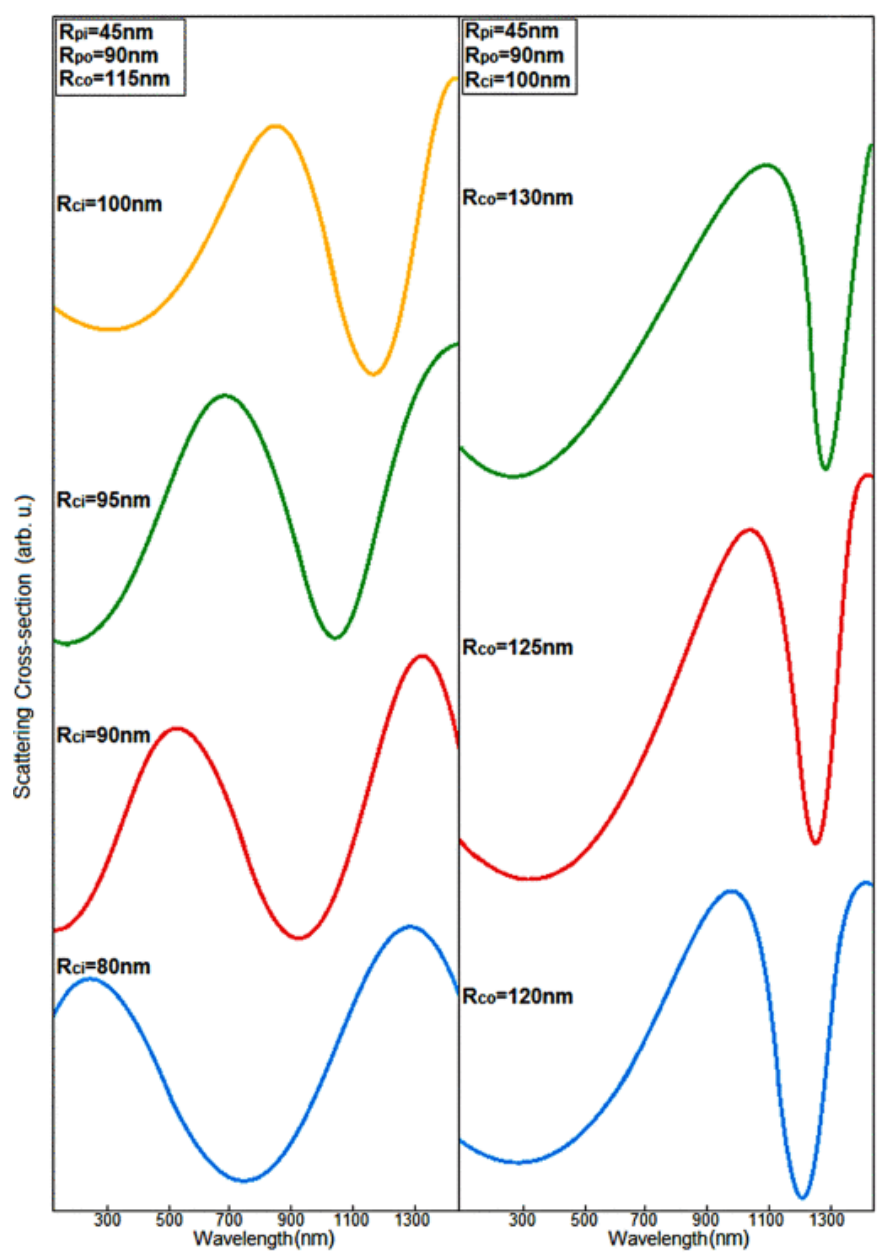

(a)

(b)

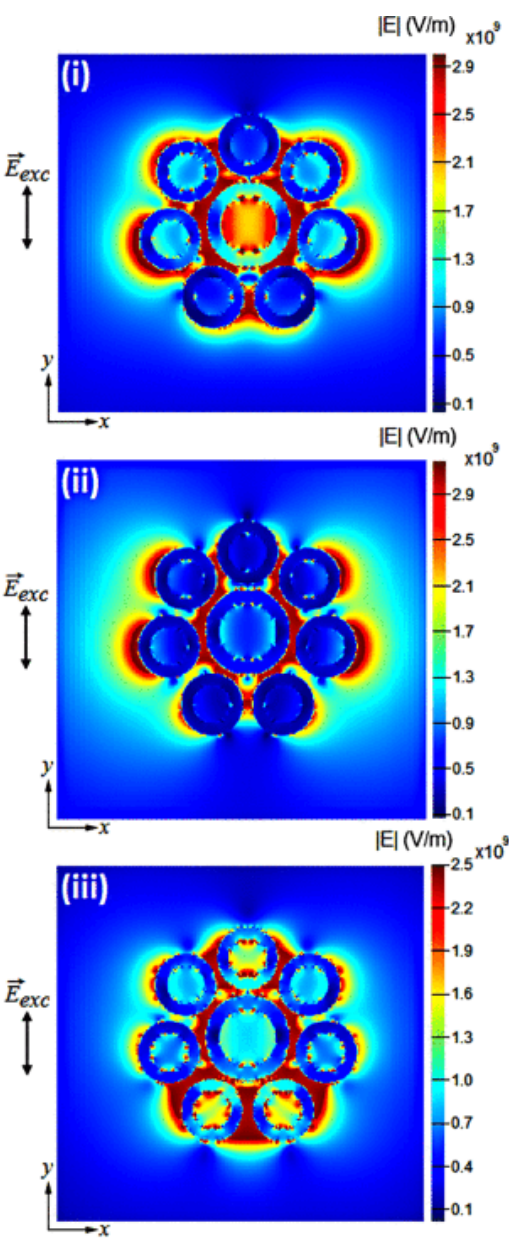

(c)

Figure 3.8. The plasmonic responses for the octamer cluster composed of Au nanoshells. a) The scattering spectra for the octamer assembly, while the inner radius of central particles is variant. b) The spectral responses for the octamer cluster composed of Au shells under transverse electric polarization mode, while the outer radius of central particles is variant. c) The two-dimensional snapshot of plasmon resonance excitation inside the nanoshells and its coupling between proximal particles. 
the position of the Fano minimum to the longer wavelengths and the narrowness enhances (see Fig. 3.8a). On the other hand, by keeping other geometrical components size unchanged, and increasing the outer radius leads to more enhancement in the linewidth of Fano dip and minor red-shift in the position of the Fano dip (see Fig. 3.8b). Figure 3.8c exhibits the E-field maps for the plasmon hybridization in the octamer cluster and formation of hotspots at the Fano dip frequency under $x$-polarized beam illumination.

In continue, we demonstrate the plasmonic response and the corresponding charge distribution at the position of bright mode and Fano dip for various structures, verifying the in-phase and out-of-phase oscillation of electrons at the superradiant bright and Fano mode, respectively. These examples contain the oscillation of charges in both simple and complex, symmetric and antisymmetric nanostructures, giving further insight into the
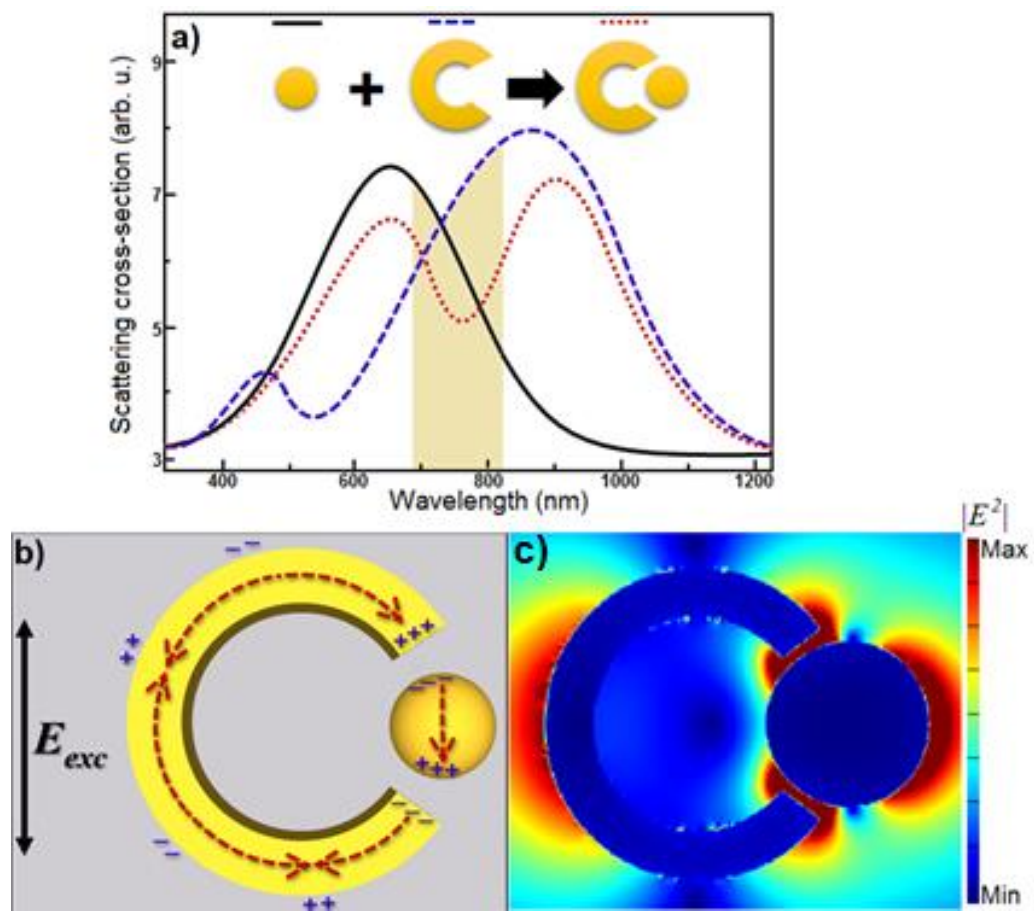

Figure 3.9. Plasmonic Response of a single-stone ring-shapes structure. a) Scattering crosssection for the formation of Fano dip. b), and c) Charge distribution and E-field maps at the Fano dip position, respectively. 

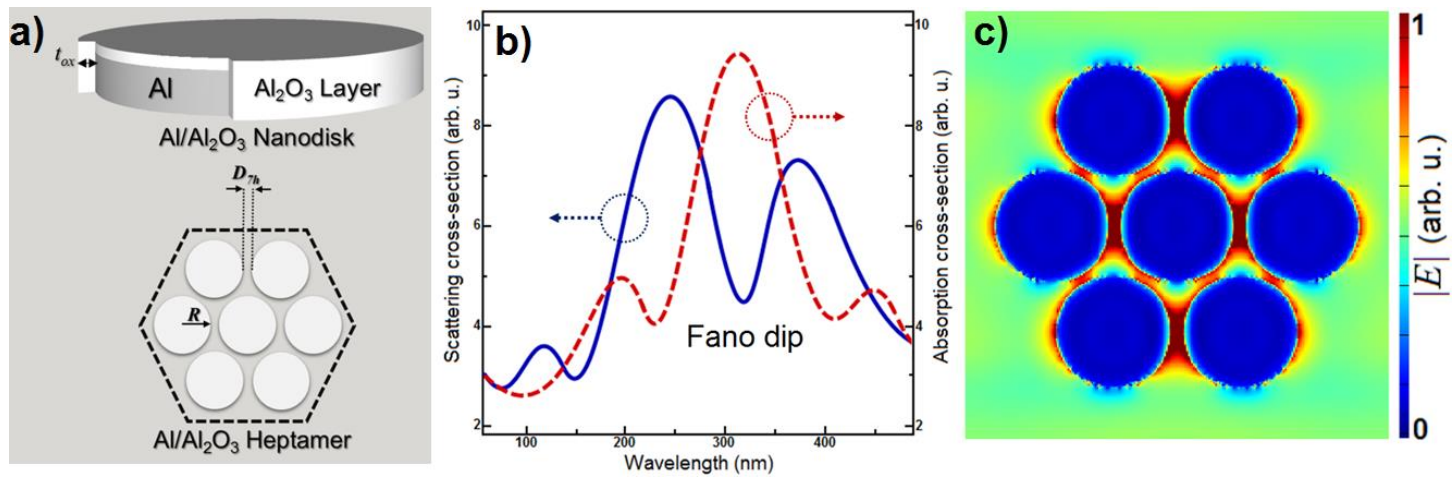

Figure 3.10. Plasmonic Response of an Al heptamer. a) Schematic of the Al heptamer with a coverage of oxide layer around nanodisks. b) Scattering and absorption cross-section for the formation of Fano dip. c) E-field maps at the Fano dip position.

Fano resonance formation mechanism. This proves how the charge distribution destructively perturbs across the structures, consistent with hybridization theory. Figure 2.9 demonstrates the spectral properties of a single-stone ring-shaped nanostructure composed of a splitted nanoring and a nanodisk [43]. As a simple nanostructure, using a step-by-step method, we plotted the formation of Fano-like lineshape. This approach allows for defining the excitation of Fano-like resonant dip with high accuracy. Considering the Au nanosphere as a part of the proposed structure with the radius of 85 $\mathrm{nm}$, a distinct broad extreme appears at $\lambda=580 \mathrm{~nm}$ (solid curve in Fig. 3.9a). The other curve (dashed) is correlated with the isolated split-ring spectral response and has two distinct peaks for narrow plasmon resonant modes. The dotted curve representing the scattering cross section spectrum of the combined system includes a dip around $\lambda \sim 800$ $\mathrm{nm}$. The overlapping region is shown with the shaded area between the low energy tail of the sphere peak and the high energy of the split-ring. In this region, electric charge density plot could help to understand the behavior of the structure and also formation of Fano-like mode. Figure $3.9 \mathrm{~b}$ and 3.9c illustrate the charge density direction and E-field distribution in the proposed system, where the direction of the incident E-field is in 
opposite direction with the excited fields in the split-ring (out-of-phase regime), which is the most important requirement to generate Fano resonance dip.

\subsubsection{Fano-Resonant Al Clusters for UV band}

As another example, Fig. 3.10a exhibits the schematic picture of a seven-member heptamer assembly composed of Al nanodisks, employed for developing a photodetector device. Here, we used Al nanodisks to induce Fano resonances along the UV domain. To this end, Al nanodisks with the radius of $70 \mathrm{~nm}$ located in $12 \mathrm{~nm}$ distance from each other with the thickness of $35 \mathrm{~nm}$. Figure $3.10 \mathrm{~b}$ represents the scattering and absorption crosssections for the Al heptamer. Clearly, an antisymmetric, narrow, and tunable plasmonic Fano minimum mode is induced around $\lambda \sim 325 \mathrm{~nm}$, which is between two distinct shoulders correlating with the bonding and antibonding plasmon modes at $\lambda \sim 250 \mathrm{~nm}$ and $\lambda \sim 385 \mathrm{~nm}$, respectively. Using previously discussed geometries for the heptamer clusters, we set the $\mathrm{Al}_{2} \mathrm{O}_{3}$ layer thickness to $t_{o x}=2 \mathrm{~nm}$. In our analysis, we detected a distinct absorption extreme at the Fano dip position with a couple of absorption shoulders in the vicinity of the bonding and antibonding modes in both of the examined heptamers. The E-field map in Fig. 3.10c demonstrates the plasmon resonance hybridization and formation of hotspots in the cluster at the Fano dip wavelength. The ultrahigh sensitivity of the Fano resonances to the environmental perturbations and geometrical variations has been exploited for developing advanced and sensitive plasmonic devices such as biosensors, modulators, and nonlinear optics [11,44,45]. In addition, the strong absorption of optical power at the Fano dip frequency has led the researchers to employ this exquisite feature for developing light absorbers and photocurrent generation in several types of plasmonic devices $[46,47]$. In continue, we summarize some important 

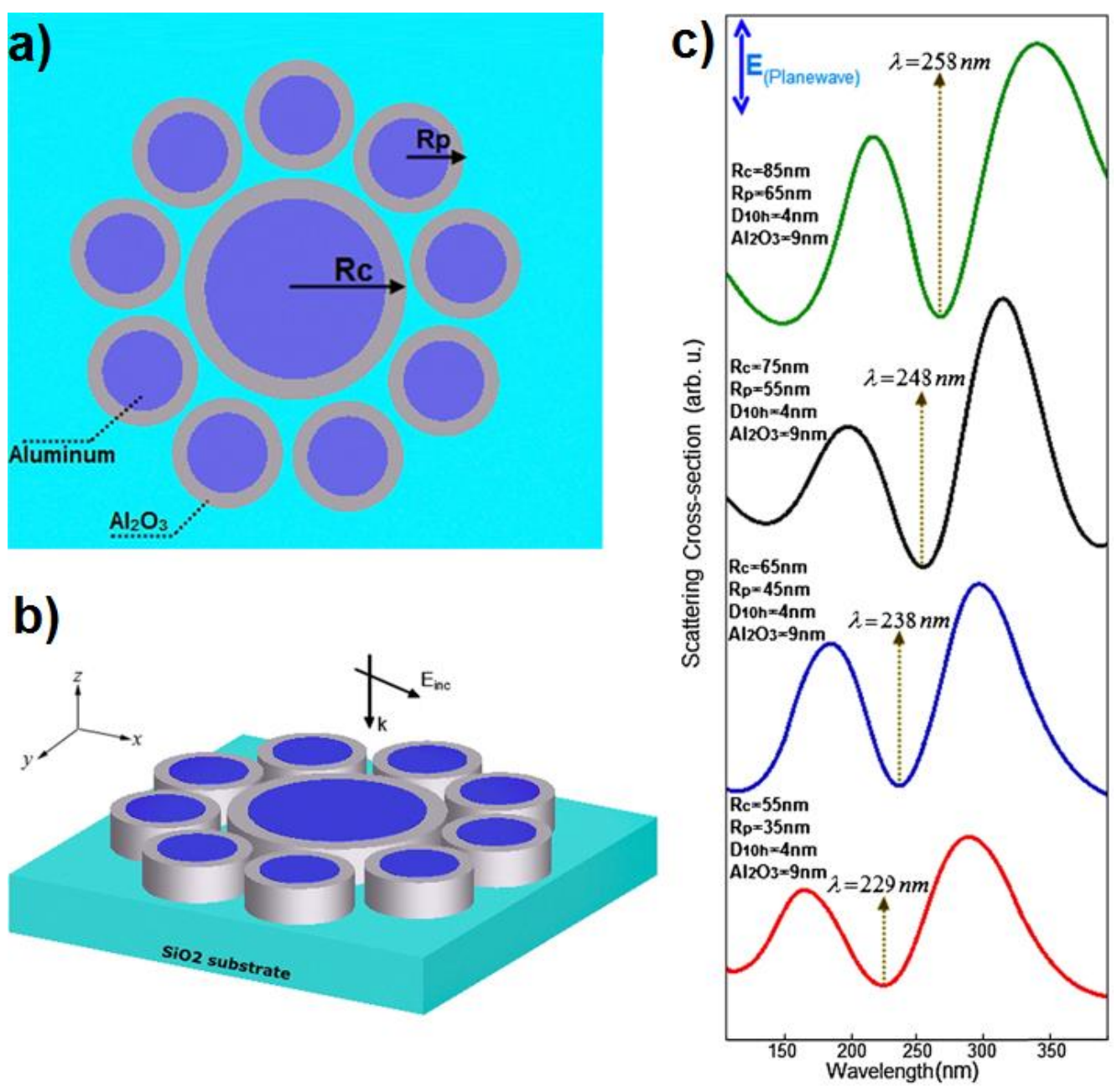

Figure 3.11. Plasmonic Response of an Al decamer. a) and b) Schematics of the Al decamer with a coverage of oxide layer around nanodisks. c) Scattering cross-section for different geometrical variations.

and fundamental applications of Fano-resonant nanostructures in advanced nanophotonic systems.

\subsubsection{Fano-Resonant Biochemical Sensors}

Much of the recent and current interest in Fano-resonant plasmonic nanosystems originates from their potential as efficient, precise, real-time, and label-free LSPR sensors $[10,12]$. The highly complex interference phenomena underlying the excitation of Fano modes in plasmonic structures are extremely sensitive to the environmental refractive 

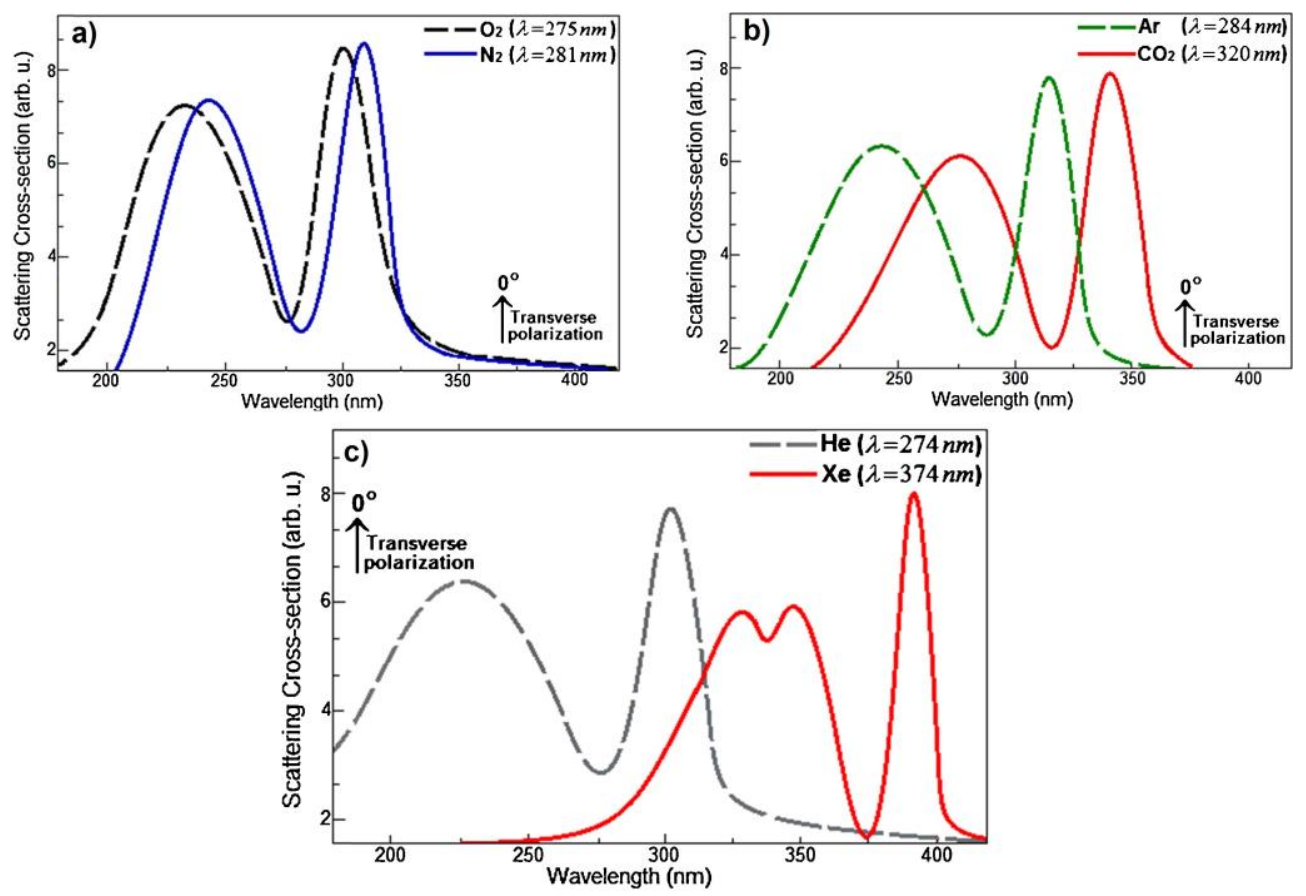

Figure 3.12. Numerically calculated scattering spectral responses for the decamer cluster, while the refractive index of the environmental medium is variant. a) The position of the FRs to the presence of $\mathrm{N}_{2}$ and $\mathrm{O}_{2}$ are evaluated. b) The position of the FRs for the presence of Ar and $\mathrm{CO}_{2}$ is determined. c) The position of the FRs for the presence of $\mathrm{He}$ and Xe is drawn.

index variations. Possessing typically narrow linewidth allows for accurate measurement of minor Fano lineshape shift due to changes in the dielectric properties of the nanostructure medium. The sensitivity of LSPR of a plasmonic nanosystem evaluates by figure of merit (FoM). This parameter defines as the ratio of the plasmon energy shift per refractive index unit change in the surrounding medium, divided by the width of the spectral feature or lineshape [48]. In other words, the FoM can be calculated by dividing the sensitivity of the structure by the resonance shift $(\Delta \lambda)$. As a practical example, we calculated the sensitivity and accuracy of a plasmonic nanosystem for the presence of various gases in the surrounding medium of a Fano-cluster using numerical methods [49]. Figures $3.11 \mathrm{a}$ and $3.11 \mathrm{~b}$ show schematic pictures of a ten-member decamer assembly composed of $\mathrm{Al}$ nanodisks with a few nanometers of $\mathrm{Al}_{2} \mathrm{O}_{3}$ oxide layer around the nanodisks. The plasmonic properties of the system are shown in Fig. 3.11c for various 
geometrical dimensions, specified inside the profile. Obviously, a distinct Fano dip is appeared at the UV band and slightly red-shifted to the lower energies by varying the corresponding geometrical sizes. The unique sensitivity of the induced Fano dip is employed for the detection of various gases. It is well-accepted that plasmonic nanostructures can be tailored to sense the gas analytes based on refractive index adsorption-dependent mechanism [50]. Accordingly, Jackšič et al. [50] have qualitatively demonstrated the performance of plasmonic sensors capable to sense the environmental refractive index perturbations based on adsorption technique. Considering the refractive index fluctuations through the surrounding medium of the structure, thus, the refractive index alterations are pertinent to the number of adsorbed molecules.

Using the proposed decamer nanosystem in this study, we try to present a simpler and effective method to sense the subtle refractive index variations based on Fano resonance behavior. To this end, the appeared Fano minimum in the UV spectrum is exploited to sense subtle variations in the refractive index of the ambient perturbations, while the dielectric substances in the ambient are gases, such as nitrogen $\left(\mathrm{N}_{2}\right)$, oxygen $\left(\mathrm{O}_{2}\right)$, carbon dioxide $\left(\mathrm{CO}_{2}\right)$, argon $(\mathrm{Ar})$, helium (He), and xenon (Xe). The challenging issue here is the small differences between the refractive indices of these materials which makes the identification of these elements highly difficult. Thus to sense the presence of these gases, we have to yield Fano minimum in the UV spectrum based on a plasmonic Fanoresonant decamer. Therefore, illuminating the examined assembly by an incident plane wave source (with the transverse polarization mode), and exposing the structure by each one of the gases, we are able to calculate and draw the scattering spectral profile numerically based on FDTD method, as exhibited in Fig. 3.12. To provide more clear and 

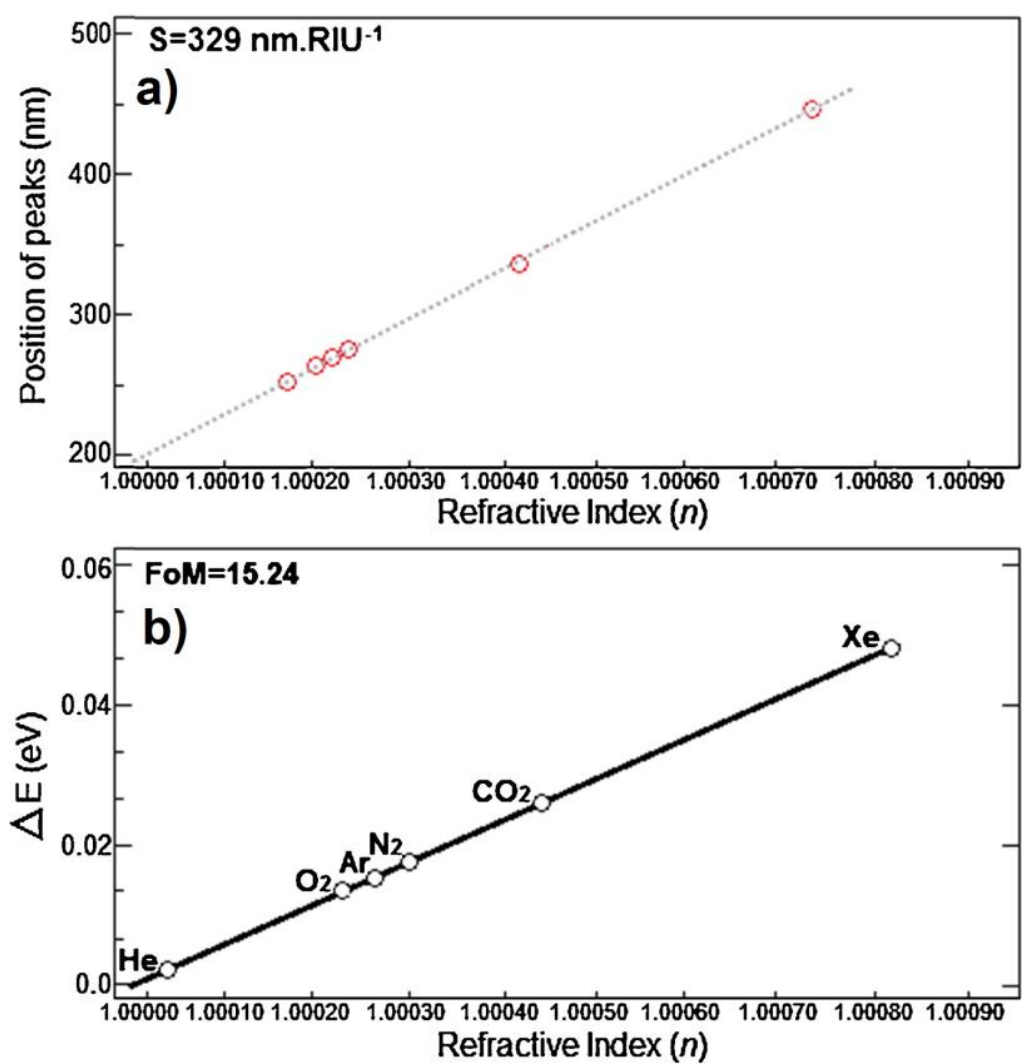

Figure 3.13. Sensitivity measurement of the plasmonic gas sensor. a) A linear plot of sensitivity for the position of plasmon resonance peaks over the refractive index variations of the ambient for an Al-based decamer. b) Linear plot for the figure of merit is depicted in the presence of various gases and quantified for the proposed compositional decamer for the presence of various gases.

definitive results, we demonstrated each one of the diagrams separately for two different gases with exact diagrams that illustrate the slight movements in the Fano dip and its quality as well. Figure 3.12 a reveals excitation of pronounced Fano minima at $\lambda \sim 281 \mathrm{~nm}$ and $275 \mathrm{~nm}$ for the presence of nitrogen $\left(\mathrm{N}_{2}\right)$ and oxygen $\left(\mathrm{O}_{2}\right)$, respectively. It is obvious that minor differences between the refractive indices of declared gases cause difference in the position of Fano dips and also the corresponding depth. Accordingly, the induced Fano dip for the $\mathrm{N}_{2}$ with higher refractive index is deeper and red-shifted to the longer wavelengths. Next, we compared the spectral response of the decamer in presence of two other gases $\left(\mathrm{CO}_{2}\right.$ and $\left.\mathrm{Ar}\right)$ that have pronounced differences in refractive indices. Figure 
$3.12 \mathrm{~b}$ illustrates calculated scattering cross-section for the assumed regime under transverse electric polarization mode numerically. Noticing in this plot, we detected two Fano minima at $\lambda \sim 284 \mathrm{~nm}$ and $320 \mathrm{~nm}$ for the presence of $\mathrm{Ar}$ and $\mathrm{CO}_{2}$, respectively. The noteworthy point here is the dramatic depth of the Fano dip for $\mathrm{CO}_{2}$ in comparison to $\mathrm{N}_{2}$. As a result, increasing the refractive index directly yields deeper Fano minimum which provide precise detection at the different surrounding ambient. Finally, we evaluated the spectral response of the nanostructure to the presentment of the He and Xe in Fig. 3.12c. Here, two different gases with a big gap between refractive index measures are considered which verifies the subtle modifications around the decamer. As we expected, the Fano minima for the He with the smallest refractive index occurred at $\lambda \sim 192 \mathrm{~nm}$ and for the Xe the Fano position red-shifted to the $\lambda \sim 280 \mathrm{~nm}$. For the final verification of the structure to utilize in sensing of LSPR in the UV spectrum, we quantified corresponding sensitivity and FoM by plotting the linear diagrams for each one. For sensitivity determination, we plotted energy peaks positions over the refractive index variations, where for FoM definition the energy deviations over the refractive index variations are computed and depicted. Figure 3.13a depicts the sensitivity diagram for the examined decamer during exposing by various gases which is quantified as $S=329 \mathrm{~nm} \cdot \mathrm{RIU}^{-1}$. In addition, we computed the plasmon resonance energy shifts over the refractive index variations as a linear plot. Technically, dividing the plasmon energy shifts per refractive index variations of the ambient by the width of scattering peak helps to realize the required FoM numerically. For the obtained asymmetric Fano resonances in the UV spectrum, the resonance energy is defined as the middle point between the plasmon energy $(\mathrm{eV})$ of the minimum and maximum peaks. Figure $3.13 \mathrm{~b}$ depicts the linear plot of 
FoM determination and accordingly, the FoM is measured as 15.24 which proves the preciseness of the designed complex plasmonic nanostructure in sensing the minor alterations in the refractive index of environmental gas substances. The same strategy can be utilized for detection of liquids or chemical components. Plasmonic biochemical and biological detractors based on Fano resonances have been employed for several practical applications with different accuracy. Here, we propose and describe the sensing

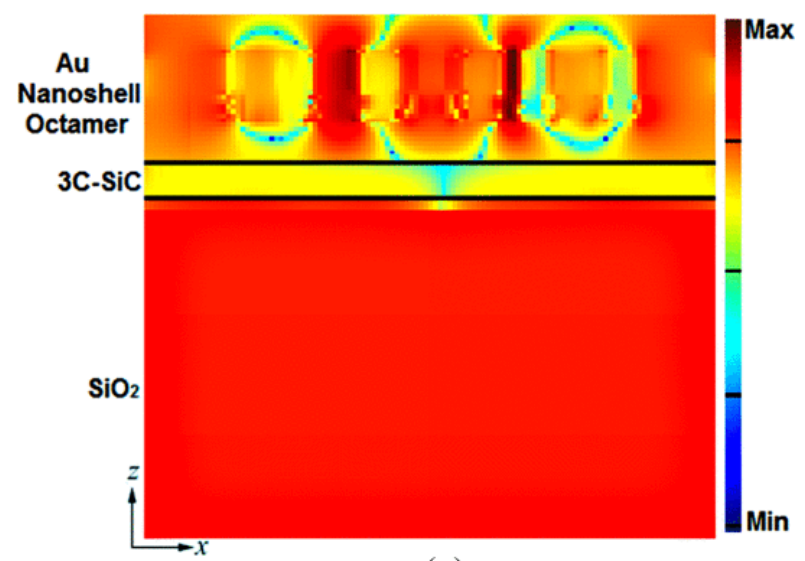

(a)

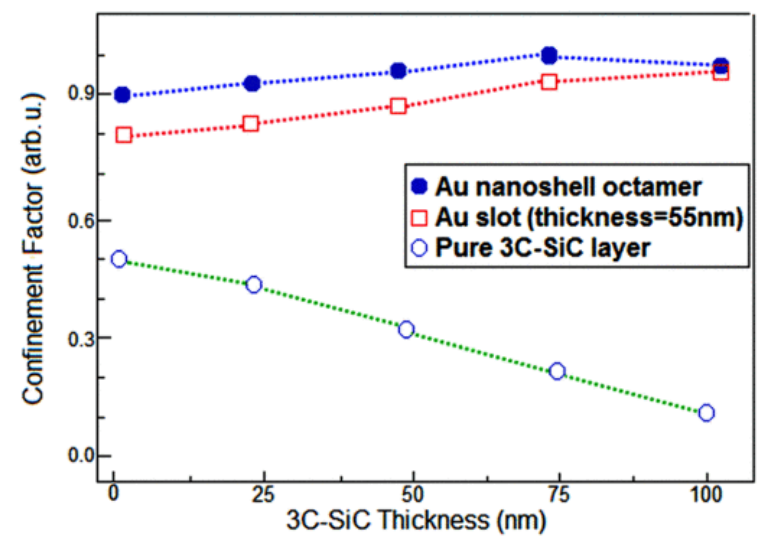

(b)

Figure 3.14. a) The cross-sectional snapshot of the transverse electric mode distribution in a proposed multilayer nanocluster-based sensor, while the refractive index of the medium is $n=$ 1.44. A low leakage of the confined field is observable between $\mathrm{SiC}$ and $\mathrm{SiO}_{2}$ layer due to high confinement of light by cluster. b) Calculated confinement factor for the multilayer structure over the thickness of $\mathrm{SiC}$ layer, while the size of nanocluster is unchanged. Also, this parameter has been compared for a structure composed of Au slot and without plasmon resonance property regimes. 
properties of a Fano-resonant nanoshell cluster on a multilayer substrate composed of $\beta$ $\mathrm{SiC} / \mathrm{SiO}_{2} / \mathrm{Si}$ for advanced sensing applications [10]. The schematic picture and the plasmonic response of the structure were plotted and explain in previous section (see Figs. 3.7 and 3.8). Here, the narrowness of Fano dip and also the strong field confinement due to the specific properties of the substrate allows for precise sensing of the environmental perturbations. In practical biochemical sensing applications, perturbations in the environmental refractive index causes to blue- or red-shift of Fano dip. In this regime, excitation of the presented plasmonic nanostructure by a plane wave source and immersing it in a dielectric liquid, we would be able to probe and investigate its plasmonic response by sketching the scattering cross-sectional profile as a function of the refractive index. Considering Lambert-Beer's law for the chemical sensing systems, distributing the optical energy along the nanostructure, a large amount of incident light can be absorbed by a simple waveguide substrate such as $\mathrm{SiO}_{2}$. As a results, using multicomponent structures would be helpful to reduce the amount of this destructive influence [41]. Pandraud et al. [51] have proved that the absorption of optical energy in waveguide systems can be described as:

$$
\alpha=\frac{1}{f l c_{e}} \log \frac{E_{0}}{E_{a}}
$$

where $\alpha$ is the absorption coefficient, $f$ is the confinement factor, $l$ is the length of the substrate waveguide, $c_{e}$ is the concentration of sensing environment, which is a variable parameter based on refractive index modifications, and $E_{0}$ and $E_{a}$ are the incident light intensity (amplitude) without and with energy absorption in the sensing environment. Here, two mechanisms were employed to define the accuracy of the proposed plasmonic 
sensor by quantifying the confinement factor of the structure [52] and plotting the corresponding FoM. We first use the confinement factor measurement to evaluate the effect of multilayer structure on this parameter, help us to determine the exact thicknesses of the layers. Then, we quantified corresponding FoM for the structure numerically by plotting the plasmon resonance energy alterations $(\Delta E(\mathrm{eV}))$ across the ambient refractive index $(n)$ variations. To estimate the confinement factor, we used following method to set the appropriate geometrical dimensions for substrate layers: It is shown that the sensitivity of a simple multilayer sensor is proportional to " $l f \alpha$," or in other words, dividing the ratio of absorbance variations $(\Delta A)$ with the concentration perturbations $\left(\Delta c_{e}\right)$ in the environmental condition yields the sensitivity of the plasmonic structure [53]. Previous studies have verified that using multilayer component and depositing metallic layer above the substrate as a sensing zone is a promising approach to improve the sensitivity of the structure due to the light confinement by metallic layer [54]. Here, for sensing part of the proposed multilayer structure, we used a Fano-resonant nanocomplex to provide strong confinement of light and localization of excited plasmon resonance modes via strong localization and hybridization of plasmon resonances, resulting strong and sharp Fano minimum. Figure 3.14a exhibits a cross-sectional snapshot for the EM field confinement between $\beta$-SiC substrate and nanoshell octamer under illumination of transverse polarized beam. The geometrical dimensions for the cluster are based on the values used in the prior section and the default thickness for the $\beta$-SiC layer is set to 85 $\mathrm{nm}$. By varying the thickness of the $\beta$-SiC substrate, we calculated the confinement factor and depicted the results in Fig. 3.14b. Numerically calculated confinement profile verifies the point that for the presence of the metallic nanoshell octamer, the maximum of 


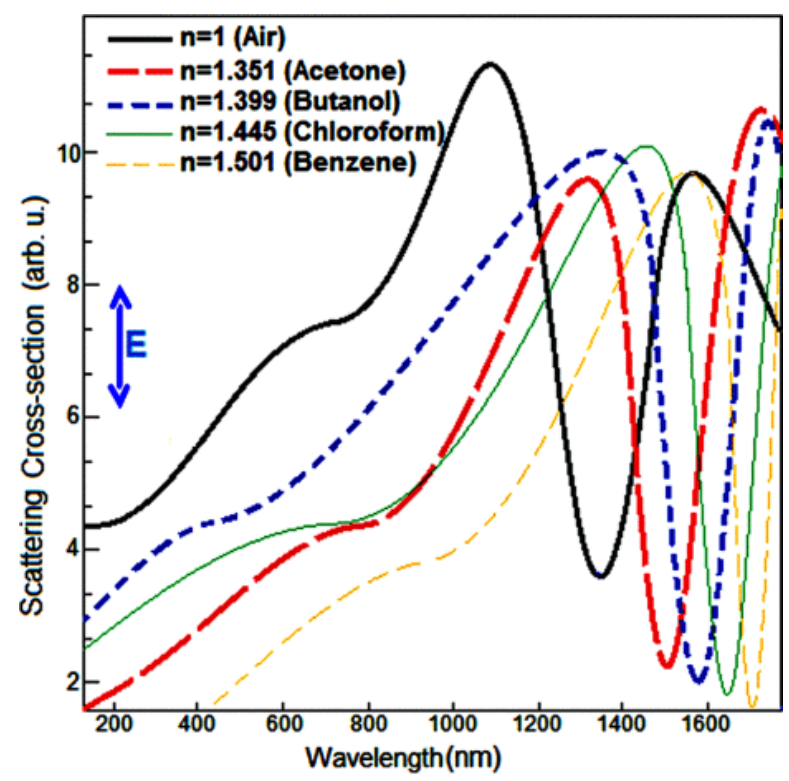

(a)

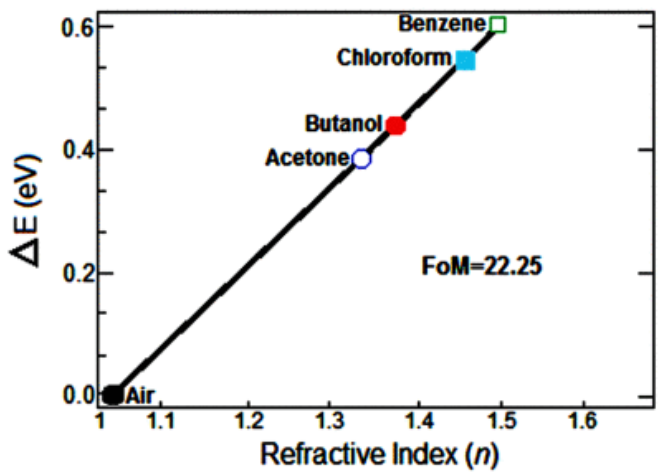

(b)

Figure 3.15. Chemical sensing properties of sensors. a) Calculated scattering spectra for the proposed nanosensor while immersing by mentioned liquids. The Fano dip red-shifts by increasing the refractive index of the medium and becomes narrower and deeper. b) Quantified FoM for the plasmonic sensor by sketching the linear fit of plasmon energy $(\Delta E)$ differences over the refractive index $(n)$ alterations.

confinement factor is achievable for the $\beta$-SiC with a thickness of $75 \mathrm{~nm}$. The figure also compares the confinement factor for the presence of simple Au layer with a thickness of $55 \mathrm{~nm}, \mathrm{Au}$ nanoshell cluster, and non-surface plasmon (or pure $\beta$-SiC) regimes, numerically. As it is obvious, employing a cluster with the height of $h=60 \mathrm{~nm}$, the structure shows superior behavior in comparison to the other investigated structural alternatives and provides a confinement factor of 0.992 for a $\mathrm{SiC}$ layer with the thickness 
of $75 \mathrm{~nm}$. For the case of using an Au slot with a thickness of $55 \mathrm{~nm}$, the confinement component is lower than the cluster-based configuration reaching to a maximum value of 0.951 for a $\beta$-SiC layer with a thickness of $115 \mathrm{~nm}$. Noticing in depicted snapshot in Fig. 3.14a, increasing the thickness of the $\beta$-SiC layer improves the confinement of plasmon resonance modes and prevents field escaping to the isolation section $\left(\mathrm{SiO}_{2}\right)$ of the multilayer structure. Finally, for the pure $\beta$-SiC or non-surface plasmon resonance regime, the situation is worse and the confinement factor is lower than $<0.52$. The confinement factor decreases by increasing the $\mathrm{SiC}$ layer thickness exponentially due to high absorption of optical energy by the substrate layer, led to undesirable losses. The noteworthy point here is the high confinement factor in the range of $0.912-0.992$, achieved for the proposed configuration with various thickness of SiC layer. This short range of variation in is an advantage for the fabrication processes, making the performance of the structure relatively insensitive to the subtle variations in the thickness of semiconductor layer.

To determine the performance of the proposed nanostructure for practical sensing applications, we investigated changes in its plasmonic response when the refractive index (n) in the surrounding medium is changed. Immersing the multilayer structure with $\mathrm{Au}$ nanoshell cluster in various liquids with different refractive indices, we measured the scattering spectra as a function of refractive index numerically. To this end, we used following liquids: $\mathrm{C}_{4} \mathrm{H}_{10} \mathrm{O}$ (Butanol) $n=1.399, \mathrm{CH}_{3} \mathrm{COCH}_{3}$ (Acetone) $n=1.351, \mathrm{CHCl}_{3}$ (Chloroform) $n=1.445$, and $\mathrm{C}_{6} \mathrm{H}_{6}$ (Benzene) $n=1.501$. Figure $3.15 \mathrm{a}$ exhibits the computed scattering cross-sectional profile for the proposed structure for varying the refractive index of the ambient. Increasing the refractive index of the surrounding medium red- 
shifts the Fano resonance position to the longer spectra, while the Fano dip becomes narrower and deeper. For instance, a pronounced Fano minimum is observed at $\lambda \sim 1380$ $\mathrm{nm}$ for the air ambience and on the other hand, for the benzene medium the Fano dip is red-shifted to $\lambda \sim 1690 \mathrm{~nm}$. Using this profile, we determined the sensitivity of the LSPR for the studied nanostructure by calculating the FoM. To quantify the FoM, we determined the ratio of the antisymmetric Fano resonance energy $(\Delta E(\mathrm{eV}))$ shift per refractive index ( $n$ ) unit variations of the environmental medium. To this end, finding the midpoint of the resonance energies of the initial and last Fano dips, we calculated the change of the resonant energy in response to the ambient index change. This dependence is plotted in Fig. 3.15b with a linear fit. Finally, we divided the slope of linear fit by a Fano line width $(0.102 \mathrm{eV})$ and found the FoM as 22.25 for the proposed structure. In the next section, we present numerical studies for the Fano-resonant nanoparticle clustersmediated UV photodetector device as part of this dissertation work.

\subsubsection{Fano-Resonant Plasmonic UV Photodetectors}

In recent years, there has been growing interest for plasmonic optoelectronic devices (i.e. transistors, photodetectors, light harvesters) for a wide spectral range covering $\mathrm{THz}$ to visible frequencies $[55,56]$. In all these works, several strategies have been carried out to enhance the photocurrent, responsivity, and quantum efficiency of the optoelectronic devices. Recently, applying the Fano effect in developing optoelectronic devices has received growing attention due to unique features of this resonant moment. As strategic devices for the next-generation advanced nanophotonic technologies, plasmonic photodetectors are promising optoelectronic devices with a wide range of applications from sensing to filtering and switching $[55,57,58]$. In the past decade, several schemes 
have been used to enhance the efficiency and responsivity of plasmonic photodetectors. For instance, graphene plasmonics has been introduced as a reliable approach to optimize the absorption of metal-semiconductor-metal (MSM) photodetectors including Schottky contacts across the optical domain to the MIR [59]. Moreover, plasmonic nanoparticles with absorptive characteristics (Ohmic losses) have widely been utilized to improve the spectral response of detectors [60]. In the previously reported works, several techniques were used to enhance the detection performance of plasmonic photodetectors such as improving the Schottky barrier height at the metal-semiconductor interface, which provides a wider depletion region and excitation of surface plasmon resonances based on collective, coherent hot electron oscillations [61].

Comparing various types of plasmonic photodetectors for various ranges of spectrum, the UV detectors are very useful for applications in UV astronomy, environmental monitoring, missile warning, and biotechnology and medicine. However, in spite of the extensive researches, the UV photodetectors suffer from dissipative losses, large dark currents, limited responsivity and quantum efficiency [62]. To address these challenges and improve the performance of the plasmonic UV detectors, two major methods have been proposed: (1) Avalanche multiplication, and (2) photoconductive gain [62]. However, high responsive gallium nitride $(\mathrm{GaN})$-based avalanche detectors suffer from an increased noise. On the other hand, the photoconductive UV detectors are slow and noisy. As another solution, GaN-based UV photodetectors with Ag plasmonic nanoparticles have been introduced to enhance the responsivity [63]. The major problem correlating with this method is the performance of utilized metals for UV bandwidth. As we discussed in the previous sections, the plasmon resonances in the subwavelength 

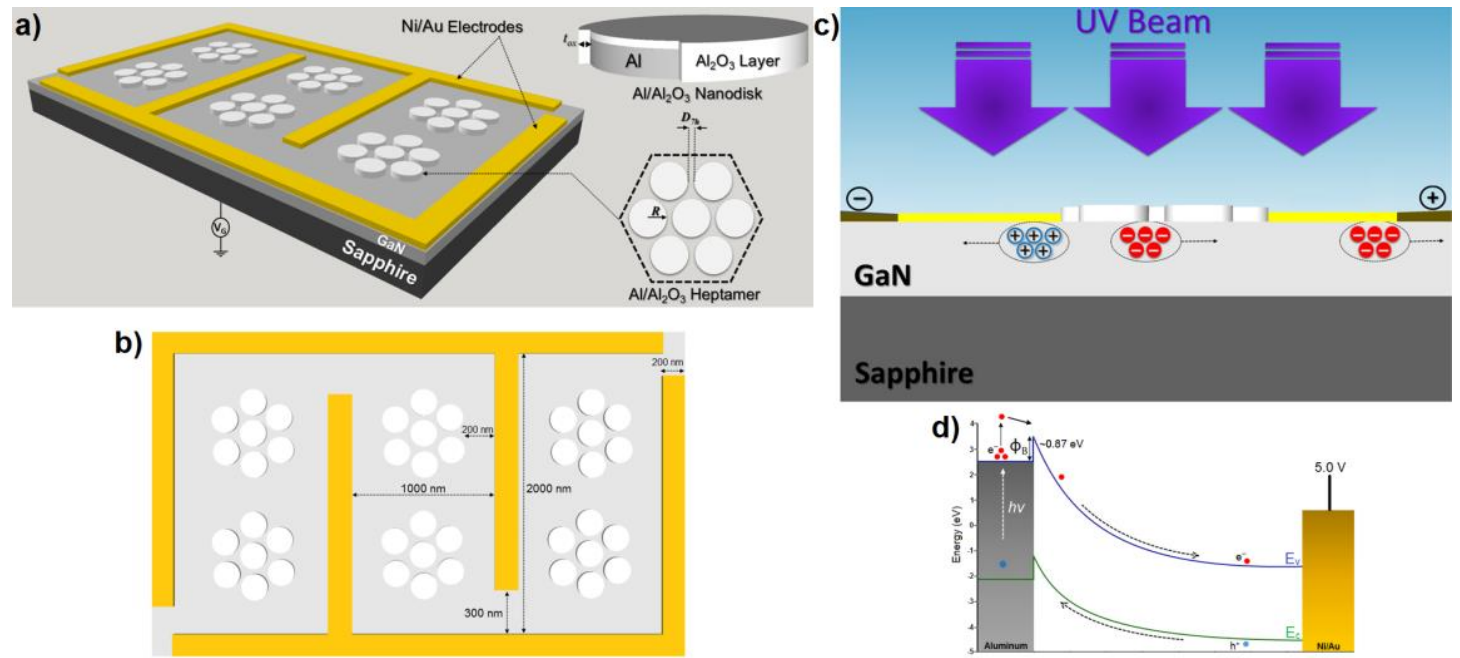

Figure 3.16. Aluminum plasmonic photodetector. a) Schematic of the plasmonic photodetector composed of $\mathrm{Al}$ nanodisk clusters deposited on GaN-sapphire substrates. The inset are the definitions for a nanodisk and a heptamer cluster with geometrical parameters. b) A top-view of the photodetector with the geometrical dimensions identification. c) The cross-sectional view of the hot electron generation and transform under the Al-based nanodisk clusters at the GaN-metal interface. d) Schematic band diagram for the Al-GaN interface, showing the carrier formation mechanism in the device.

structures based on conventional noble metals (e.g. $\mathrm{Au}, \mathrm{Ag}$, and $\mathrm{Cu}$ ) can be effectively tuned across the visible wavelengths to the NIR. However, extending these plasmonic properties into the UV spectrum is highly challenging due to the intrinsic limitations in the chemical characteristics of the used metals. For instance, Ag shows dramatic degradation in plasmonic properties because of rapid oxidation and Au suffers from the interband transitions in the UV band. Lately, Al, Rhodium (Rh), Gallium (Ga), Chromium (Cr), and Indium (In) have been introduced as potential plasmonic materials for the UV spectrum [64]. Al has extensively been utilized for designing light harvesting devices, nanoantennas, cathodoluminescence spectroscopy, and antireflective surfaces [65], in spite of the inherent and rapid oxidation. Al also shows significant EM field localization because of its low screening $\left(\varepsilon_{\infty} \approx 1\right)$ in comparison to gold $\left(\varepsilon_{\infty} \approx 9\right)$ and $\mathrm{Ag}$ $\left(\varepsilon_{\infty} \approx 5\right)$. In addition, $\mathrm{Al}$ has high electron density since a single $\mathrm{Al}$ atom contributes three 
electrons compared to a single electron per atom for $\mathrm{Au}$ and $\mathrm{Ag}$ [65]. Due to the negligible influence of interband transitions in $\mathrm{Al}$ across the UV spectrum, therefore, the geometry of nanoscale structure plays a major role in decaying plasmons and generation of photoexcited hot carriers during light-matter interactions. Here, we propose and extensively analyze a novel device based on plasmonic $\mathrm{Al} / \mathrm{Al}_{2} \mathrm{O}_{3}$ nanoparticle assemblies integrated into a GaN UV photodetector. To this end, we utilized seven-member heptamers with the symmetry of a benzene molecule as Fano-resonant plasmonic nanoclusters. All of the $\mathrm{Al}$ particles are deposited between Nickle $(\mathrm{Ni}) / \mathrm{Au}$ fingers on a GaN active layer grown on a sapphire substrate. The presented results show that nanoplasmonic $\mathrm{Al}$ assemblies could generate hot electrons to enhance the absorption via inducing the Fano resonance modes across the UV spectrum. The proposed structure could realize the UV photodetectors with a significantly improved responsivity.

Both radiative and non-radiative excitation of plasmons in metallic structures and their drastic decay leads to generation of hot carriers at the metal-semiconductor interfaces [66]. The surface modes are important to attain the plasmon resonant behavior and hot carrier distribution at the metal-semiconductor interfaces. In the proposed system here, the confined plasmons also give rise to the formation of dynamic hotspots with extremely intense local fields in the capacitive regions between the closely-coupled particles. Assuming electrons have an isotropic momentum distribution, approximately half of the photoexcited electrons are expected to be transported to the Al-GaN interface. Due to the continuous distribution of highly energetic hot electrons in the Al nanostructures [67], hence, we expect large number of charges to reach the metalsemiconductor interface compared to the conventional noble metals. Figure 3.16a shows 
a three-dimensional schematic of the proposed plasmonic UV detector (not to scale). The device comprises arrays of $\mathrm{Al} / \mathrm{Al}_{2} \mathrm{O}_{3}$ heptamer nanoclusters between two Ni/Au fingers (electrodes) deposited on an undoped n-type GaN epilayer with the thickness of $4 \mu \mathrm{m}$ which is grown on a sapphire substrate. The inset figure shows the geometry of the heptamer assembly. The space between two proximal heptamers is set to $250 \mathrm{~nm}$ to prevent any destructive optical interference between the scattered fields associating with hybridized modes arising from the nearby antennas. In Fig. 3.16b, we depict the important geometrical dimensions for the metallic electrodes, the overall size of the proposed photodetector, and the distance between two fingers. Using the plasmon hybridization theory to analyze closely packed nanoscale assemblies [15], the plasmon responses of various types of Al-based nanodisk oligomers and monomers have already been investigated numerically and analytically in the previous sections. It is also shown that $\mathrm{Al}$ nanodisk heptamer antennas with a thin oxide layer $(2-25 \mathrm{~nm}$, depends on the size of consisting particles) can be tailored to support strong plasmonic FR mode across the near-UV $(\lambda \sim 350 \mathrm{~nm})$ band. However, this wavelength is not unique and the position of Fano minimum can be tuned via modifications in the geometrical, chemical and environmental parameters of the assembly. In the proposed plasmonic UV detector, we used nanodisks with following geometrical dimensions: the radius of nanodisks is $R=70$ $\mathrm{nm}$ with the thickness of $t=35 \mathrm{~nm}$ separated with the offset gap of $D_{7 h}=12 \mathrm{~nm}$. It should be noted that while the thickness of the oxide layer around nanoparticles is varied the size of offset gap is kept fixed to satisfy the required near-field coupling strength. To provide a detailed study and compare the effect of Al heptamer arrays on the responsivity and performance of the structure, we also demonstrate the spectral response of the structure 
without presence of antennas on $\mathrm{GaN}$ as the non-plasmonic regime. The frequencydependent absorption mechanism of the proposed plasmonic UV detector is based on the hybridized plasmon resonant modes due to the interaction of an incident beam with the metallic antenna. The maximum absorption can be achieved at the spectral position of the antisymmetric Fano lineshape, because of the suppression of the scattering bright resonant dipolar peak by narrow antibonding dark mode. Such a significant absorption leads to generation of large number of hot carriers at the metal-dielectric interface, which are transferred to semiconductor surmounting the Schottky barrier and collected by the electrodes resulting a remarkable photocurrent and hence, high responsivity. Figure 2.16c displays a two-dimensional (xz-view) cross-sectional schematic of the proposed UV detector displaying the hot electron transport in the GaN layer to the adjacent electrodes. It is well-understood that in a metal-semiconductor system, reduced electron-electron scattering in the metallic part of the nanoantenna increases the number of hot electrons transferred to the semiconductor layer $[66,68]$. This ultrafast transition of plasmonic charges leads to accumulation of more hot electrons and sweeping them before immediate recombination. Figure $2.16 \mathrm{~d}$ demonstrates the schematic band diagram profile for the proposed plasmonic device, showing the carrier formation and transition mechanisms and sweeping opposite charges to the nearby electrodes. When a bias is applied between the metallic contacts one forward and one reverse biased Schottky junctions are formed. The large electric field in between, results in sweeping of the photogenerated hot electrons to the positive electrode and thus producing a photocurrent. However, due to losses via back-scattering, inelastic collisions, and heat energy conversion (internal damping), not all of the photoexcited electrons are injected to the 


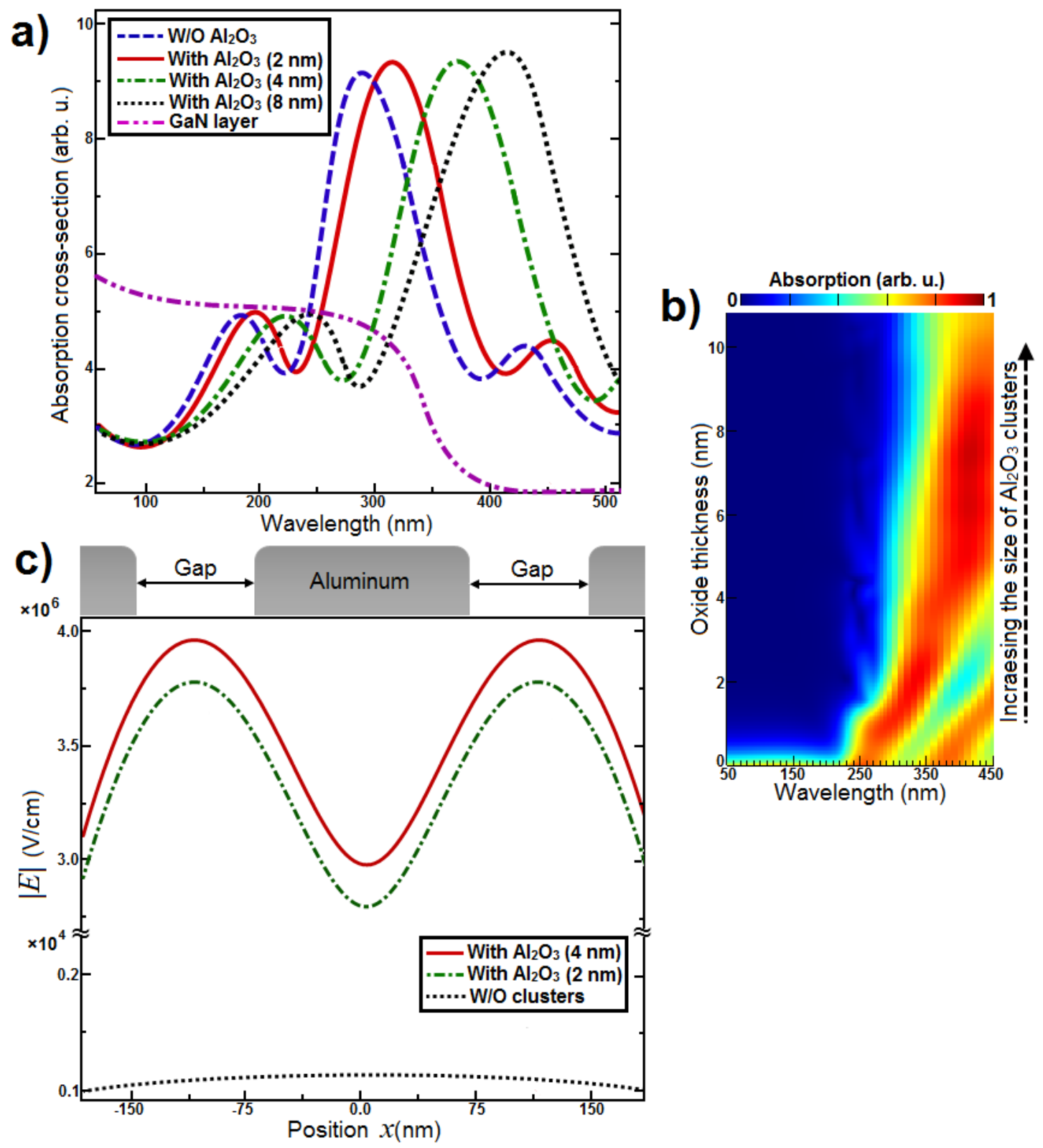

Figure 3.17. The plasmon responses for the UV photodetector. a) The absorption spectra for heptamer clusters deposited in GaN epilayer with variant oxide thickness and without metallic heptamers. b) E-field enhancement diagram for the UV device with and without Al clusters. c) Numerically plotted absorption spectra for the oxide layer thickness as a function of incident UV beam in a heptamer nanocluster.

semiconductor [68]. Thus we have to consider only the hot carriers within the mean-free path (MFP) length $\left(l_{p}\right)$ distance from the interface for transferring to the semiconductor over the Schottky barrier [69]. In this approach, the optically driven electrons are excited 
from the energy states below the Fermi level to the higher energy levels, and once they arrive at the interface with an energy larger than the Schottky barrier height get injected to the GaN. Experimental results show that MFP for electrons is strongly energydependent and minor perturbations in energy level results significant changes [70]. In our analysis we assumed $l_{p}=25 \mathrm{~nm}$ for electrons $5 \mathrm{eV}$ above Fermi level energy as reported in Ref. [70].

Considering plotted band diagram for the $\mathrm{Al}-\mathrm{GaN}-\mathrm{Ni} / \mathrm{Au}$ interface, the electrical simulation results verify formation of a Schottky barrier with the height of $\Phi_{B}=0.87 \mathrm{eV}$. In this regime, the decayed plasmons result hot electrons that are arrived to the interface with higher energies more than $\sim 0.87 \mathrm{eV}$ are able to pass the barrier and transit to reach the biased electrode. In the examined device, hot electron generation rate $\left(G_{h e}\right)$ by $\mathrm{Al}$ nanoantennas at the Fano dip wavelength due to photoexcitation can be calculated using [71]: $G_{h e}=P C_{a b s}(\lambda) / \hbar \omega A_{h}$, where $P$ is the incident light power $(20 \mu \mathrm{W}), C_{a b s}(\lambda)$ is the absorption cross section as a function of resonant wavelength, and $A_{h}$ is the metallic nanoantenna area and found to be $G_{h e}=5 \times 10^{17} \mathrm{~s}^{-1}$. Then, the electron concentration can be calculated as: $n_{e}=\tau G_{h e} / A_{h}$, where $\tau$ is the relaxation time, which assessed to be $0.825 \times 10^{-6} \mathrm{~s}$, using following method: For the examined plasmonic nanocluster the relaxation time can be written as [72]:

$$
\tau=\sqrt{t_{t r}^{2}+\left(R_{L} C\right)^{2}}
$$

where $R_{L}$ is the $\mathrm{Al}$ antenna resistance, and $C$ is the gate-interface capacitance that can be defined as below: 


$$
C=\frac{A_{h} \varepsilon_{0}\left(\varepsilon_{G a N}+1\right)}{L+W}\left(\frac{\pi}{4 \operatorname{Ln}\left(\frac{8}{\pi}+\frac{L}{W}\right)}\right)
$$

where $\varepsilon_{0}$ is the permittivity of the vacuum., $L$ and $W$ are the pitch and electrode width, respectively. Therefore, for $L=500 \mathrm{~nm}, W=200 \mathrm{~nm}, R_{L}=250 \mathrm{k} \Omega$, and $\varepsilon_{G a N}=9.7$, the capacitance is calculated as $330 \mathrm{pF}$. The approximate electron concentration at the AlGaN interface is defined as $n_{e}=1.04 \times 10^{17} \mathrm{~cm}^{-2}$. Comparing hot electron generation rate and the associated concentration in the proposed system with $\mathrm{Al}$ nonamers and gratings for the same purpose [71], we realized a significant enhancement due to inherent and remarkable absorptive behavior of $\mathrm{Al}$ across the $\mathrm{UV}$ spectrum as well as continuous electron energy distribution [73].

For conventional semiconductor layers that have broadly been utilized for photocurrent generation in designing plasmonic devices (e.g. Si, CdSe, etc.), the carrier lifetime is in the range of $\sim 100 \mu$ s. Long carrier lifetime prevents immediate recombination and facilitates generation of large photocurrent. In contrast, the carrier lifetime and recombination process for $\mathrm{UV}$-compatible $\mathrm{GaN}$ around is in the range of a few nanoseconds. In this regime, extremely short transition time (in the range of a few picoseconds) is required to overcome the immediate recombination of the carriers. Using experimentally and theoretically obtained values for the saturation velocity $\left(V_{\text {sat }}\right)$ in ntype GaN [74], the transition time $\left(t_{t r}\right)$ can be determined by: $t_{t r}=L / V_{\text {sat }}$, where $L$ is the pitch. As a general rule for III-V materials, the bulk saturation velocity in high-field mobility can be defined by modeling as a function of lattice temperature given by [75]: 


$$
V_{\text {sat }}(T)=\frac{V_{\text {sat }}(T=300 \mathrm{~K})}{(1-A)+\left(\frac{T}{300 \mathrm{~K}}\right) A}
$$

where $V_{\text {sat }}$ is the saturation velocity at the lattice temperature $(T=300 \mathrm{~K})$, and A represents the temperature coefficient, showing the strong dependency of the various materials which are included in the mechanism (Monte Carlo simulations model). Therefore, for the saturation velocity of $10^{5} \mathrm{~cm} / \mathrm{s}$, with the pitch of $500 \mathrm{~nm}$, the transition time is calculated as $5 \times 10^{-12} \mathrm{~s}(5 \mathrm{ps})$ which is extremely short $\left(t_{t r}<<\tau_{n}\right)$ compared to the carrier lifetime in $\mathrm{GaN}$ which is around $6.5 \mathrm{~ns}$ [76]. Consequently, large number of electrons can be collected before they recombine resulting photocurrent with gain. Additionally, for the uncovered parts of the photodetector, the incident photons with the energies larger than the bandgap of $\mathrm{GaN}$ can also be absorbed and generate electron-hole pairs. These electron-hole pairs in the uncovered parts will be added to the hot electron pairs of clusters and contribute to the photocurrent (see Fig. 2.16d). The fast relaxation and transition times constitute the base for very fast temporal response for the proposed devices. We estimated that the rise and fall times are in the sub-microsecond range using the standard methods [77].

By considering the plasmonic response of an Al heptamer cluster (see Fig. 2.10), next, we analyze the electric response of the structure. The absorption spectra for an isolated Al nanodisk heptamer on a glass host is plotted in Fig. 3.17a. Increasing the thickness of the oxide layer leads to enhancements in the absorption efficiency due to formation of narrower Fano minimum resulted by the suppression of the scattering extreme by antibonding dark resonant mode. This phenomenon includes a red-shift in the 
position of the peaks to the longer wavelengths. The reason originates from the strong EM field hybridization of plasmonic resonances in large size heptamer clusters. As a result, deeper Fano minimum in the extinction profile can be induced, including a significant enhancement in the ratio of the absorbed power. Noticing in the absorption profile in Fig. 3.17a, for the heptamer assembly with thicker oxide layer, the peak of the absorption is shifted to the visible spectrum that is not desired for our UV photodetector. In addition, for the ideal case, for an entirely $\mathrm{Al}$ cluster without oxide layer $\left(t_{o x} \sim 0 \mathrm{~nm}\right)$, a noticeable extreme is appeared at the short wavelengths around $\lambda \sim 280 \mathrm{~nm}$, close to deepUV band. For $t_{o x}=2 \mathrm{~nm}$ and $4 \mathrm{~nm}$ two absorption extremes are obtained at $\lambda \sim 320 \mathrm{~nm}$ and $345 \mathrm{~nm}$, respectively, which have almost equal amplitude. This profile also shows the absorption spectra for the UV detector without presence of nanoparticle clusters. Noticing in the corresponding curve, due to the absence of metallic components and plasmonic effects, we observe only the natural absorption of incoming UV beam by GaN substrate, which is reduced dramatically after UV band $\lambda>400 \mathrm{~nm}$. Figure $3.17 \mathrm{~b}$ represents numerically obtained absorption spectra for $\mathrm{Al}_{2} \mathrm{O}_{3}$ thickness variations as a function of incident beam. The absorption ratio increased significantly including a red-shift to the visible spectrum by increasing the thickness and the entire size of the heptamer. The enhancement of the electric field $(|\mathrm{E}|)$ at the gap spots between central nanodisks of the heptamer cluster is shown in Fig. 3.17c. Significant enhancements of the electric field is observed at the offset spots between Al nanodisks because of hybridization and strong confinement of the plasmonic resonant modes. Comparing two types of antennas with different oxide thicknesses, a slight difference in the enhancement is noticed. This effect can be described by the effect of oxide layer thickness on the Bruggeman dielectric 

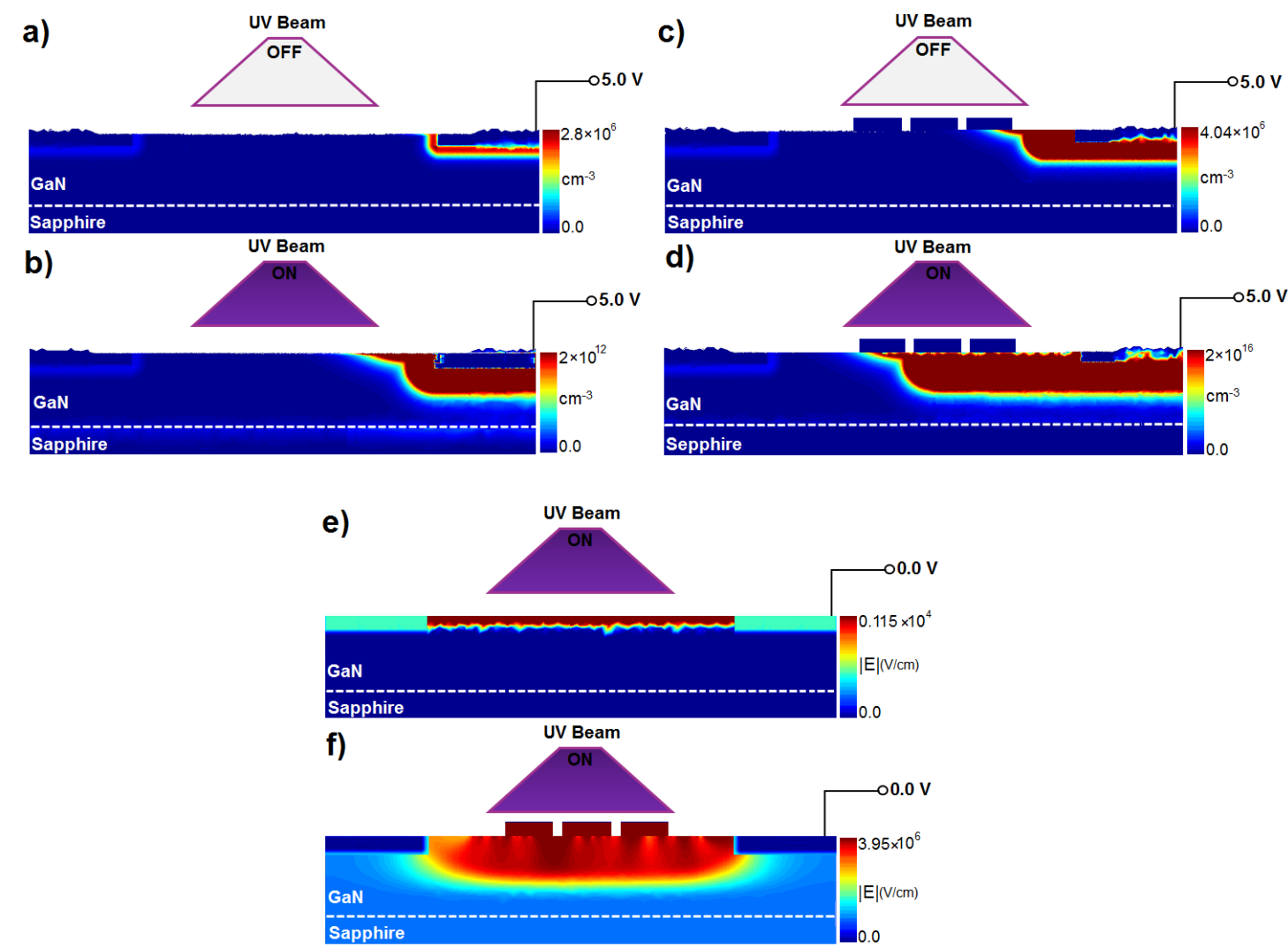

Figure 3.18. Electrical response of plasmonic UV photodetector. a, b) Carrier concentration for the detector system without heptamers with bias $(5.0 \mathrm{~V})$, while the UV light is in OFF and ON states, respectively. c, d) Carrier concentration for the system with heptamers with bias (5.0 V), while the UV light is in OFF and ON states, respectively. e, f) E-field enhancement map for the device with and without clusters, while the UV light is $\mathrm{ON}$ and bias is $0.0 \mathrm{~V}$.

function of the entire composite $\mathrm{Al}$ antenna, yielding different real and imaginary permittivities at different wavelengths. Hence, modifying the oxide thickness can lead to severe changes in the spectral response of the structure, as shown in the preceding profiles. This plot also shows that no distinct shoulder is observed in the electric field profile at the illumination spots for the absence of metallic heptamers and therefore the absence of the plasmonic effects. For the case without the heptamers, a thin layer of electric field appears at the surface of the GaN (with the magnitude of $1.15 \times 10^{5} \mathrm{~V} / \mathrm{cm}$ ). While for the plasmonic case, a much larger electric field is monitored below the cluster due to hybridization of plasmons (with the magnitude of $3.95 \times 10^{6} \mathrm{~V} / \mathrm{cm}$ ). 

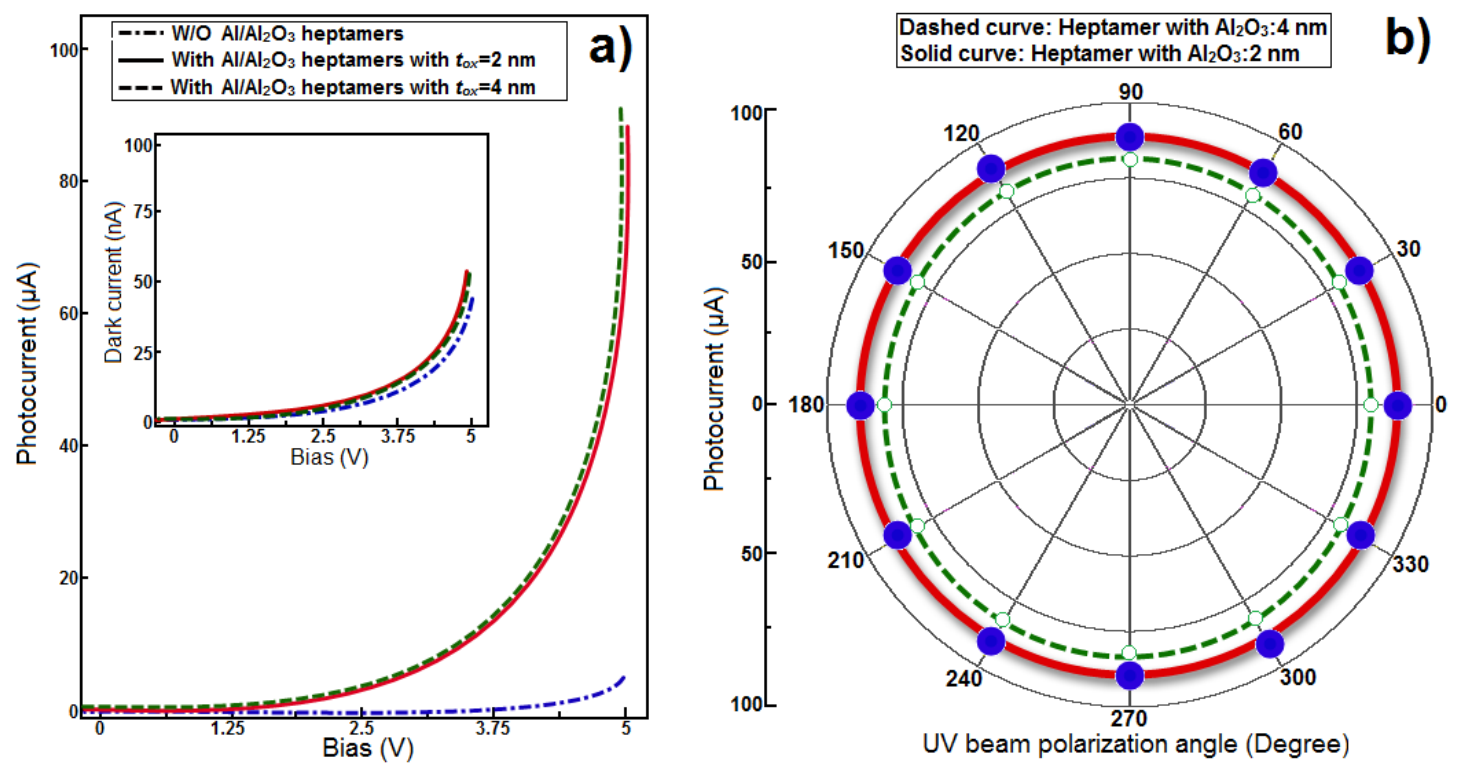

Figure 3.19. Electrical response for the UV photodetector. a) Numerically achieved photocurrent-voltage (I-V) curves for two different oxide thicknesses of a heptamer cluster and without heptamers. The inset is the dark current-voltage (I-V) curves for the non-plasmonic and plasmonic UV photodetector for two different oxide thicknesses at $\lambda=325 \mathrm{~nm}$ and $335 \mathrm{~nm}$ for $t_{o x}=2 \mathrm{~nm}$, and $4 \mathrm{~nm}$, respectively. b) Polarization-independency of the generated photocurrent (blue-spheres) of the device for the polarization angle variations of the incident UV beam.

Figures $3.18 \mathrm{a}$ and $3.18 \mathrm{~b}$ demonstrate the electron concentration for the proposed UV photodetector device without presence of $\mathrm{Al}$ heptamers, while the bias is applied $(5.0 \mathrm{~V})$ and the light source is OFF and ON, respectively (these states are indicated inside the corresponding profile in Fig. 3.18). By applying both bias and UV beam, comparing to the absence of the beam (Fig. 3.18a), a noticeable electron concentration is obtained under the electrodes (Fig. 3.18b), resulting a photocurrent. On the other hand, by adding metallic nanoscale heptamers between electrodes, we observed a dramatic enhancement in the concentration of carriers resulted by the metallic clusters, as shown in Figs. 3.18c and 3.18d, respectively. To show the effect of plasmonic clusters on carrier generation, we used Al nanoparticles with the oxide coverage of $t_{o x}=2 \mathrm{~nm}$. In this regime, the generated large carrier concentration causes Schottky barrier lowering which could 

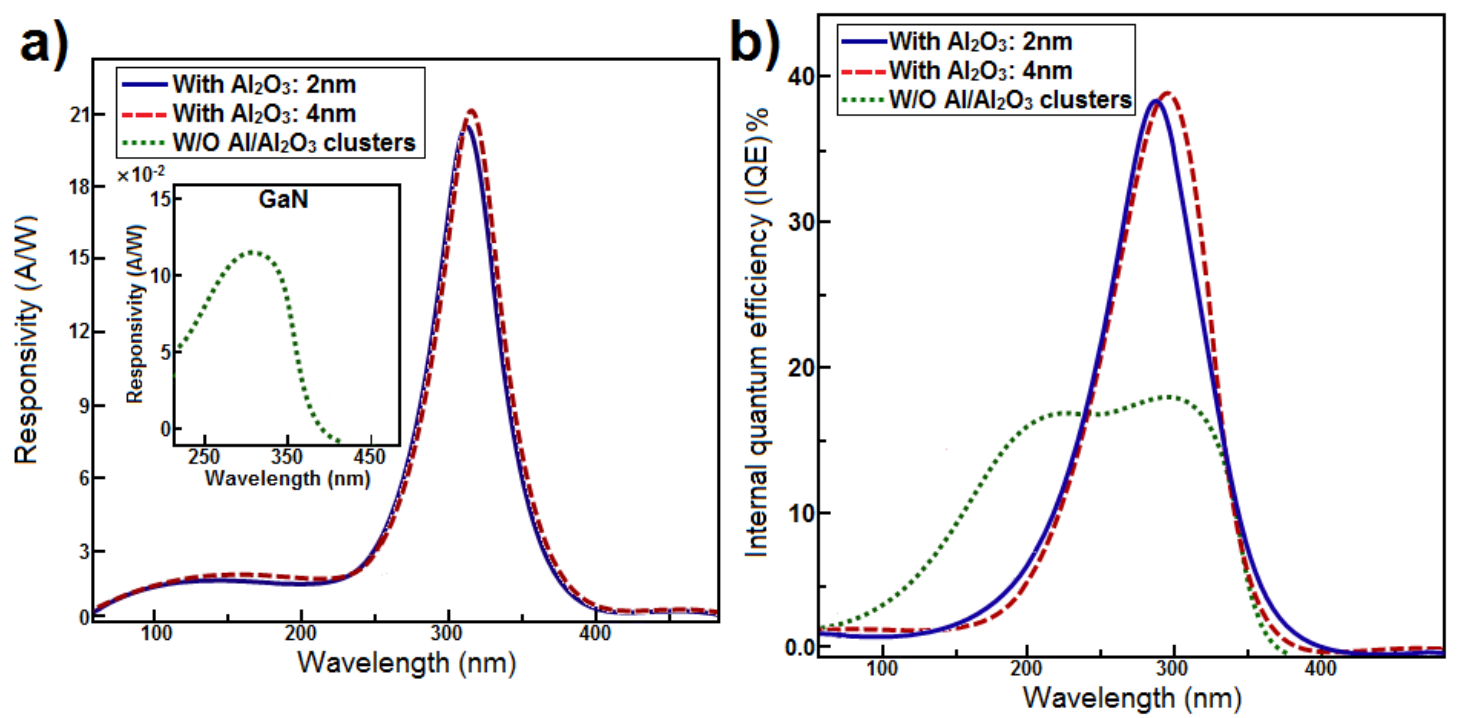

Figure 3.20. The spectral responses for the UV detector in both non-plasmonic and plasmonic regimes, with variant $\mathrm{Al}_{2} \mathrm{O}_{3}$ thicknesses of heptamer clusters. a) Responsivity profile under $5.0 \mathrm{~V}$ applied bias. Inset is the responsivity profile for the non-plasmonic regime. $\mathrm{b}$ ) The IQEs for different regimes of the UV detector.

contribute to the enhancement of the photocurrent. The above comparison between nonplasmonic and plasmonic UV detectors can be further illustrated by plotting corresponding E-field maps for the excited electric field at the surface of the GaN, below the antennas, as shown in Figs. 3.18e and 3.18f, where the effect of plasmonic antennas in formation of a large field at the $\mathrm{GaN}-\mathrm{Al}$ interface is obvious.

Further, we study the electrical response of the plasmonic GaN photodetector. Figure 3.19a illustrates the current-voltage $(I-V)$ characteristic calculated for the peak of the absorption profile along with the one for the device without plasmonic assemblies. In the calculated response, the voltage is changed between $0 \mathrm{~V}$ to $5.0 \mathrm{~V}$. For a bias of $5.0 \mathrm{~V}$ the plasmonic detector with the heptamer arrays with $t_{o x}=2 \mathrm{~nm}$ and $4 \mathrm{~nm}$, yield the photocurrents of $88.56 \mu \mathrm{A}$ and $90.25 \mu \mathrm{A}$, respectively. For the non-plasmonic case (absence of metallic nanoparticle clusters), the photocurrent is found as $1.72 \mu \mathrm{A}$ under 
5.0 V bias. Dramatic enhancement in the photocurrent due to the plasmonic heptamers is clearly visible. The inset of Fig. 3.19a shows the extracted dark current as a function of the bias voltage, which reaches to $47.95 \mathrm{nA}, 52.5 \mathrm{nA}$ and $55.25 \mathrm{nA}$ for the non-plasmonic case without the heptamers, $t_{o x}=2 \mathrm{~nm}$ and $4 \mathrm{~nm}$, respectively, under $5.0 \mathrm{~V}$ bias. Figure $3.19 \mathrm{~b}$ shows the photocurrent as a function of the polarization angle of the incoming light. In the plotted figure, hallow and solid circles represent the calculated photocurrents for different incident polarization modes in two types of heptamers with different oxide thicknesses. It is observed that the response of the proposed structure is insensitive to the variations of the polarization angle of the incident EM energy due to the inherent symmetry of the molecular heptamer cluster. In addition, besides the ability to support pronounced Fano dip at the UV spectrum, it should be noted that antisymmetric structures with more complex geometries cannot provide such a high and polarizationindependent absorption spectra [78].

Figure 3.20a represents the spectral response of the proposed UV detector with and without $\mathrm{Al}$ antenna arrays, where the thickness of the oxide layer is changed and the bias is kept as $5.0 \mathrm{~V}$. For the plasmonic regime, the responsivity peaks are at $\lambda \sim 325 \mathrm{~nm}$ and $\lambda \sim 330 \mathrm{~nm}$, and the cutoff wavelengths here is at $\lambda \sim 335 \mathrm{~nm}$ and $\lambda \sim 345 \mathrm{~nm}$, for $t_{o x}=2 \mathrm{~nm}$ and $4 \mathrm{~nm}$, respectively. The peak responsivity $\left(R_{p h}\right)$ corresponds to the position of Fano dip of heptamers. At the peaks of the curve for the heptamer with $t_{o x}=2 \mathrm{~nm}$ and $4 \mathrm{~nm}$, the responsivity of the proposed plasmonic UV photodetector exceed $20.8 \mathrm{~A} / \mathrm{W}$ and 21.9 A/W, respectively. This outcome shows the superior responsivity of the examined UV detector in comparison to analogous nanoscale devices [79]. On the other hand, for the non-plasmonic case, we observed a conventional responsivity with a distinct shoulder at 
the UV spectra in the range of $\lambda \sim 300 \mathrm{~nm}$ to $350 \mathrm{~nm}$, where at the highest peak this parameter is measured as approximately $0.13 \mathrm{~A} / \mathrm{W}$ (the inset diagram in Fig.3.20a). Using the calculated responsivity data for the proposed plasmonic UV photodetector, we extracted the $\mathrm{EQE}$ for the structure in two different regimes by employing the conventional equation: $\mathrm{EQE}=h c R / e \lambda$, where $h$ is the Planck's constant, $c$ is the velocity of light, $e$ is the electron charge, $R$ is the responsivity of the device, and $\lambda$ is the wavelength of the incoming optical power. The calculated EQE for the UV detector in non-plasmonic case is $64.5 \%$ while $\mathrm{EQE}$ is $8065 \%$ and $8116 \%$ for the devices with the presence of $\mathrm{Al}$ clusters with $t_{o x}=2 \mathrm{~nm}$ and $4 \mathrm{~nm}$, respectively. This dramatic enhancement in the responsivity and efficiency of the device during transition from non-plasmonic to the plasmon regime originates from the generation of hot carriers due to strong hybridization of plasmons at resonant frequencies. As the other important parameter, we estimated the IQE of the proposed UV photodetector, which is the number of the produced charge carriers per incident photon and can be calculated using the computed photocurrent profile as well as the incoming photon energy flux on the subwavelength heptamer antennas. The absorbed power by the structure is given by: $P_{a b s}=-0.5 \omega_{p}|E|^{2} \operatorname{Im}\left(\varepsilon_{e f f}\right)$, where $|E|$ is the amplitude of the incident electric field, and $\varepsilon_{e f f}$ is the effective permittivity of the semiconductor substrate and metallic heptamer that are contributed in the absorption mechanism. Accordingly, the number of the absorbed photons is given by:

$$
N_{p h}=\frac{-0.5 \omega_{p}|E|^{2} \operatorname{Im}\left(\varepsilon_{e f f}\right)}{\hbar}
$$

On the other hand, using the equation above, we define the IQE: 


$$
I Q E=\frac{\text { Number of hot electrons/Sec }}{N_{p h}}
$$

Figure $3.20 \mathrm{~b}$ exhibits numerically obtained IQE for the proposed device as a function of the incident UV light, where the peaks for $t_{o x}=2 \mathrm{~nm}$ and $4 \mathrm{~nm}$ with the values of $38 \%$ and $40 \%$, respectively are induced at the FR dip positions. It is also worth noting that at these short wavelengths, generation of hot electrons by metallic nanodisk heptamers has an undeniable impact in having such a large photocurrent as well as a significant IQE. We also calculated corresponding IQE for the UV detector without nanodisk clusters displayed in the profile with dotted curve as $15.6 \%$. A comparison of the performance of all the examined regimes for the proposed UV photodetector shows that inducing the plasmonic effect via $\mathrm{Al}$ clusters enhances the responsivity and photocurrent of the device with the expense of having a few nano-amperes dark current. The response of the plasmonic UV photodetector is comparable with the more complex designs that have been proposed, such as coupling of plasmons between $\mathrm{Al}$ and $\mathrm{ZnO}$ nanoparticles, or using multilayer substrate to enhance the electron-hole confinement to improve generated photocurrent. Finally, we estimated the corresponding gain $\left(\Gamma_{p h}\right)$ of the investigated photodetector using:

$$
\Gamma_{p h}=\frac{\mathrm{R}_{p h}}{I Q E}\left(\frac{h c}{q \lambda}\right)
$$

where $q$ is the elementary charge, and $c$ is the velocity of light. The corresponding gain is found to be $\Gamma_{\mathrm{ph}}=2.1 \times 10^{2}$ for the Al cluster with the oxide thickness of $t_{o x}=2 \mathrm{~nm}$. In this section by proposing a recent study for the application of Fano resonances to develop optoelectronic devices such as plasmonic photodetectors. By calculating the important 
parameters for the proposed photodetector, we proved its superior performance and quality in comparison to analogous devices without plasmonic structures. Possessing high responsivity, quantum efficiency, internal gain, and significant photocurrent across the UV spectrum make this structure as a potential platform for designing and fabricating optoelectronic UV devices for several sensing applications. This study also shows the significant impact of the enhanced absorption cross-section at the Fano dip wavelength in achieving high responsivity and photocurrent.

\subsection{References}

1. Ahmadivand, A., \& Golmohammadi, S. (2014). Optimized plasmonic configurations: adjacent and merging regimes between a symmetric couple of Au rod/shell nanoarrangements for LSPR sensing and spectroscopic purposes. Journal of nanoparticle Research, 16, 2491.

2. Jackson, J. D. (1999). Classical electrodynamics (Vol. 3). Wiley, New York.

3. Grahn, P., Shevchenko, A., \& Kaivola, M. (2012). Electromagnetic multipole theory for optical nanomaterials. New Journal of Physics, 14(9), 093033.

4. Lambert, R. H. (1978). Complete vector spherical harmonic expansion for Maxwell's equations. American Journal of Physics, 46(8), 849-852.

5. Bohren, C. F., \& Huffman, D. R. (1983). Absorption and scattering of light by small particles. Wiley, New York.

6. Gómez, D. E., Teo, Z. Q., Altissimo, M., Davis, T. J., Earl, S., \& Roberts, A. (2013). The dark side of plasmonics. Nano Letters, 13(8), 3722-3728.

7. Chang, Y. C., Wang, S. M., Chung, H. C., Tseng, C. B., \& Chang, S. H. (2012). Observation of absorption-dominated bonding dark plasmon mode from metalinsulator-metal nanodisk arrays fabricated by nanospherical-lens lithography. ACS Nano, 6(4), 3390-3396.

8. Mishra, Y. K., Adelung, R., Kumar, G., Elbahri, M., Mohapatra, S., Singhal, R., Tripathi, A., \& Avasthi, D. K. (2013). Formation of self-organized silver nanocuptype structures and their plasmonic absorption. Plasmonics, 8(2), 811-815.

9. Ahmadivand, A., Sinha, R., Karabiyik, M., Vabbina, P. K., Gerislioglu, B., Kaya, S., \& Pala, N. (2017). Tunable THz wave absorption by graphene-assisted plasmonic 
metasurfaces based on metallic split ring resonators. Journal of Nanoparticle Research, 19(1), 3.

10. Golmohammadi, S., Ahmadivand, A., \& Pala, N. (2015). Fano Resonances in Nanoshell Clusters Deposited on a multilayer substrate of $\beta-\mathrm{SiC} / \mathrm{SiO}_{2} / \mathrm{Si}$ to design high-quality plasmonic sensors. Journal of Lightwave Technology, 33(13), 28172823.

11. Chang, W. -S., Lassiter, J. B., Swanglap, P., Sobhani, H., Khatua, S., Nordlander, P., Halas, N. J., \& Link S. (2012). A plasmonic Fano switch. Nano Letters, 12(9), 49774982.

12. Ahmadivand, A., Karabiyik, M., \& Pala, N. (2015). Fano-like resonances in split concentric nanoshell dimers in designing negative-index metamaterials for biologicalchemical sensing and spectroscopic purposes. Applied Spectroscopy, 69(5), 563-573.

13. Liu, N., Mukherjee, S., Bao, K., Li, Y., Brown, L. V., Nordlander, P., \& Halas, N. J. (2012). Manipulating magnetic plasmon propagation in metallic nanocluster networks. ACS Nano, 6(6), 5482-5488.

14. Ahmadivand, A., \& Golmohammadi, S. (2014). Electromagnetic plasmon propagation and coupling through gold nanoring heptamers: a route to design optimized telecommunication photonic nanostructures. Applied Optics, 53(18), 38323840 .

15. Prodan, E., Radloff, C., Halas, N. J., \& Nordlander, P. (2003). A hybridization model for the plasmon response of complex nanostructures. Science, 302(5644), 419-422.

16. Prodan, E., \& Nordlander, P. J. C. P. (2004). Plasmon hybridization in spherical nanoparticles. The Journal of Chemical Physics, 120(11), 5444-5454.

17. Prodan, E., \& Nordlander, P. (2003). Structural tunability of the plasmon resonances in metallic nanoshells. Nano Letters, 3(4), 543-547.

18. Hentschel, M., Dregely, D., Vogelgesang, R., Giessen, H., \& Liu, N. (2011). Plasmonic oligomers: the role of individual particles in collective behavior. ACS Nano, 5(3), 2042-2050.

19. Nordlander, P., Oubre, C., Prodan, E., Li, K., \& Stockman, M. I. (2004). Plasmon hybridization in nanoparticle dimers. Nano Letters, 4(5), 899-903.

20. Xu, Y. L., \& Gustafson, B. Å. (2001). A generalized multiparticle Mie-solution: further experimental verification. Journal of Quantitative Spectroscopy and Radiative Transfer, 70(4), 395-419. 
21. Lassiter, J. B., Aizpurua, J., Hernandez, L. I., Brandl, D. W., Romero, I., Lal, S., Hafner, J. H., Nordlander, P., \& Halas, N. J. (2008). Close encounters between two nanoshells. Nano Letters, 8(4), 1212-1218.

22. Ahmadivand, A., Sinha, R., Pala, N. (2016). Resonance coupling in plasmonic nanomatryoshka homo- and heterodimers. AIP Advances, 6(6), 065102.

23. Ahmadivand, A., \& Pala, N. (2015). Multiple Fano resonances in plasmonic metamaterials composed of $\mathrm{Al} / \mathrm{Al}_{2} \mathrm{O}_{3}$ nanomatryushka structures. MRS Proceedings, $1788,43-48$.

24. Palik, E. D. (1998). Handbook of optical constants of solids. Academic Press, San Diego, CA.

25. Gong, H. M., Zhou, L., Su, X. R., Xiao, S., Liu, S. D., \& Wang, Q. Q. (2009). Illuminating dark plasmons of silver nanoantenna rings to enhance exciton-plasmon interactions. Advanced Functional Materials, 19(2), 298-303.

26. Sonnefraud, Y., Verellen, N., Sobhani, H., Vandenbosch, G. A., Moshchalkov, V. V., Van Dorpe, P., Nordlander, P. \& Maier, S. A. (2010). Experimental realization of subradiant, superradiant, and Fano resonances in ring/disk plasmonic nanocavities. ACS Nano, 4(3), pp.1664-1670.

27. Sancho-Parramon, J., \& Bosch, S. (2012). Dark modes and Fano resonances in plasmonic clusters excited by cylindrical vector beams. ACS Nano, 6(9), 8415-8423.

28. Ahmadivand, A., Sinha, R., \& Pala, N. (2017). Magnetic Fano resonances in alldielectric nanocomplexes under cylindrical vector beams excitation. Optics \& Laser Technology, 90, 65-70.

29. Sakai, K., Nomura, K., Yamamoto, T., \& Sasaki, K. (2015). Excitation of multipole plasmons by optical vortex beams. Scientific Reports, 5, 8431.

30. Ahmadivand, A., Gerislioglu, B., \& Pala, N. (2017). Azimuthally and radially excited charge transfer plasmon and Fano lineshapes in conductive sublayer-mediated nanoassemblies. J. Opt. Soc. Am. A, 34(11), 2052-2056.

31. Huang, J. S., Kern, J., Geisler, P., Weinmann, P., Kamp, M., Forchel, A., Biagioni, P. $\&$ Hecht, B. (2010). Mode imaging and selection in strongly coupled nanoantennas. Nano Letters, 10(6), 2105-2110.

32. Yang, S. C., Kobori, H., He, C. L., Lin, M. H., Chen, H. Y., Li, C., Kanehara, M., Teranishi, T. \& Gwo, S. (2010). Plasmon hybridization in individual gold nanocrystal dimers: direct observation of bright and dark modes. Nano Letters, 10(2), 632-637. 
33. Hao, F., Larsson, E. M., Ali, T. A., Sutherland, D. S., \& Nordlander, P. (2008). Shedding light on dark plasmons in gold nanorings. Chemical Physics Letters, 458(4), 262-266.

34. Volpe, G., Cherukulappurath, S., Juanola Parramon, R., Molina-Terriza, G., \& Quidant, R. (2009). Controlling the optical near field of nanoantennas with spatial phase-shaped beams. Nano Letters, 9(10), 3608-3611.

35. Gómez, D. E., Vernon, K. C., \& Davis, T. J. (2010). Symmetry effects on the optical coupling between plasmonic nanoparticles with applications to hierarchical structures. Physical Review B, 81(7), 075414.

36. Davis, T. J., Hentschel, M., Liu, N., \& Giessen, H. (2012). Analytical model of the three-dimensional plasmonic ruler. ACS Nano, 6(2), 1291-1298.

37. Fan, S., Suh, W., \& Joannopoulos, J. D. (2003). Temporal coupled-mode theory for the Fano resonance in optical resonators. J. Opt. Soc. Am. A, 20(3), 569-572.

38. Tribelsky, M. I., \& Luk'yanchuk, B. S. (2006). Anomalous light scattering by small particles. Physical Review Letters, 97(26), 263902.

39. Miroshnichenko, A. E., Flach, S., Gorbach, A. V., Luk'yanchuk, B. S., Kivshar, Y. S., \& Tribelsky, M. I. (2008). Fano resonances: A discovery that was not made 100 years ago. Optics and Photonics News, 19(12), 48-48.

40. Zhang, Q., Wen, X., Li, G., Ruan, Q., Wang, J., \& Xiong, Q. (2013). Multiple magnetic mode-based Fano resonance in split-ring resonator/disk nanocavities. ACS Nano, 7(12), 11071-11078.

41. Ghosh, G. (1999). Dispersion-equation coefficients for the refractive index and birefringence of calcite and quartz crystals. Optics communications, 163(1), 95-102.

42. Johnson, P. B., \& Christy, R. W. (1972). Optical constants of the noble metals. Physical Review B, 6(12), 4370.

43. Ahmadivand, A., Sinha, R., Kaya, S., Pala, N. (2016). A molecular plasmonic Fanorouter: using hotspots in a single-stone ring-like structure. Optics Communications. $367,123-129$.

44. Luk'yanchuk, B., Zheludev, N. I., Maier, S. A., Halas, N. J., Nordlander, P., Giessen, H., \& Chong, C. T. (2010). The Fano resonance in plasmonic nanostructures and metamaterials. Nature Materials, 9(9), 707.

45. Thyagarajan, K., Butet, J., \& Martin, O. J. (2013). Augmenting second harmonic generation using Fano resonances in plasmonic systems. Nano Letters, 13(4), 18471851. 
46. Qiang, Z., Zhou, W. D., Lu, M., \& Brown, G. J. (2008). Fano resonance enhanced infrared absorption for infrared photodetectors. Proc. SPIE, 6901, 69010F.

47. Vasinajindakaw, P., Vaillancourt, J., Gu, G., Liu, R., Ling, Y., \& Lu, X. (2011). A Fano-type interference enhanced quantum dot infrared photodetector. Applied Physics Letters, 98(21), 211111.

48. Hentschel, M., Saliba, M., Vogelgesang, R., Giessen, H., Alivisatos, A. P., \& Liu, N. (2010). Transition from isolated to collective modes in plasmonic oligomers. Nano Letters, 10(7), 2721-2726.

49. Ahmadivand, A., Golmohammadi, S., \& Pala, N. (2015). Fano resonances in plasmonic aluminum nanoparticle clusters for precise gas detection: Ultra-sensitivity to the minor environmental refractive index perturbations. Photonics and Nanostructures-Fundamentals and Applications, 13, 97-105.

50. Jakšić, O. M., Jakšić, Z. S., Čupić, Ž. D., Randjelović, D. V., \& Kolar-Anić, L. Z. (2014). Fluctuations in transient response of adsorption-based plasmonic sensors. Sensors and Actuators B: Chemical, 190, 419-428.

51. Lupan, O., Postica, V., Marx, J., Mecklenburg, M., Mishra, Y. K., Schulte, K., Fiedler, B., \& Adelung, R. (2017). Individual hollow and mesoporous aero-graphitic microtube based devices for gas sensing applications. Applied Physics Letters, 110(26), 263109.

52. Ricciardi, C., Bennici, E., Cocuzza, M., Mandracci, P., Bich, D., Guglielmetti, V., \& Barucca, G. (2003). Characterization of polycrystalline SiC layers grown by ECRPECVD for micro-electro-mechanical systems. Thin Solid Films, 427(1), 187-190.

53. Niehusmann, J., Vörckel, A., Bolivar, P. H., Wahlbrink, T., Henschel, W., \& Kurz, H. (2004). Ultrahigh-quality-factor silicon-on-insulator microring resonator. Optics Letters, 29(24), 2861-2863.

54. Harris, R. D., \& Wilkinson, J. S. (1995). Waveguide surface plasmon resonance sensors. Sensors and Actuators B: Chemical, 29(1), 261-267.

55. Nabet, B. (2015). Photodetectors: Materials, Devices and Applications. Elsevier, Woodhead Publishing.

56. Tsuchiya, H., kamakura, Y. (2016). Carrier Transport in Nanoscale MOS Transistors. John Wiley \& Sons.

57. Chen, Q., Chitnis, D., Walls, K., Drysdale, T. D., Collins, S., \& Cumming, D. R. (2012). CMOS photodetectors integrated with plasmonic color filters. IEEE Photonics Technology Letters, 24(3), 197-199. 
58. Zheng, B. Y., Wang, Y., Nordlander, P., \& Halas, N. J. (2014). Color-Selective and CMOS-Compatible Photodetection Based on Aluminum Plasmonics. Advanced Materials, 26(36), 6318-632.

59. Wang, X., Cheng, Z., Xu, K., Tsang, H. K., \& Xu, J. B. (2013). High-responsivity graphene/silicon-heterostructure waveguide photodetectors. Nature Photonics, 7(11), 888-891.

60. Schaadt, D. M., Feng, B., \& Yu, E. T. (2005). Enhanced semiconductor optical absorption via surface plasmon excitation in metal nanoparticles. Applied Physics Letters, 86(6), 063106.

61. Chen, H., Liu, H., Zhang, Z., Hu, K., \& Fang, X. (2016). Nanostructured photodetectors: from ultraviolet to terahertz. Advanced Materials, 28(3), 403-433.

62. Hu, G. C., Shan, C. X., Zhang, N., Jiang, M. M., Wang, S. P., \& Shen, D. Z. (2015). High gain $\mathrm{Ga}_{2} \mathrm{O}_{3}$ solar-blind photodetectors realized via a carrier multiplication process. Optics Express, 23(10), 13554-13561.

63. Weng, W. Y., Hsueh, T. J., Chang, S. J., Wang, S. B., Hsueh, H. T., and Huang, G. J. (2011). A high-responsivity GaN nanowire UV photodetector. IEEE Selected Topics in Quantum Electronics 17(4), 996-1001.

64. Ahmadivand, A., Sinha, R., Kaya, S., \& Pala, N. (2016). Rhodium plasmonics for deep-ultraviolet bio-chemical sensing. Plasmonics, 11(3), 839-849.

65. Knight, M. W., Liu, L., Wang, Y., Brown, L., Mukherjee, S., King, N. S., Everitt, H. O., Nordlander, P., and Halas. N. J. (2012). Aluminum plasmonic nanoantennas. Nano Letters 12(11), 6000-6004.

66. Knight, M. W., Sobhani, H., Nordlander, P., \& Halas, N. J. (2011). Photodetection with active optical antennas. Science, 332(6030), 702-704.

67. Bernardi, M., Mustafa, J., Neaton, J. B., \& Louie, S. G. (2015). Theory and computation of hot carriers generated by surface plasmon polaritons in noble metals. Nature Communications, 6, 7044.

68. Wu, K., Chen, J., McBride, J. R., \& Lian, T. (2015). Efficient hot-electron transfer by a plasmon-induced interfacial charge-transfer transition. Science, 349(6248), 632635.

69. Chalabi, H., Schoen, D., \& Brongersma, M. L. (2014). Hot-electron photodetection with a plasmonic nanostripe antenna. Nano Letters, 4(3), 1374-1380. 
70. Kanter, H. (1970). Slow-electron mean free paths in aluminum, silver, and gold. Physical Review B, 1(2), 522.

71. Fang, Z., Wang, Y., Liu, Z., Schlather, A., Ajayan, P. M., Koppens, F. H., Nordlander, P. and Halas, N. J., (2012). Plasmon-induced doping of graphene. ACS Nano, 6(11), 10222-10228.

72. Neamen, D. A. (2012) Semiconductor Physics and Devices: Basic Principles. (McGrew-Hill Education).

73. Sundararaman, R., Narang, P., Jermyn, A. S., Goddard III, W. A., \& Atwater, H. A. (2014). Theoretical predictions for hot-carrier generation from surface plasmon decay. Nature Communications, 5, 5788.

74. Anwar, A. F. M., Wu, S., \& Webster, R. T. (2001). Temperature dependent transport properties in $\mathrm{GaN}, \mathrm{Al}_{\mathrm{x}} \mathrm{Ga}_{1-\mathrm{x}} \mathrm{N}$, and $\mathrm{In}_{\mathrm{x}} \mathrm{Ga}_{1-\mathrm{x}} \mathrm{N}$ semiconductors. IEEE Transactions on Electron Devices, 48(3), 567-572.

75. Quay, R., Moglestue, C., Palankovski, V., \& Selberherr, S. (2000). A temperature dependent model for the saturation velocity in semiconductor materials. Materials Science in Semiconductor Processing, 3(1), 149-155.

76. Bandić, Z. Z., Bridger, P. M., Piquette, E. C., \& McGill, T. C. (1998). Minority carrier diffusion length and lifetime in GaN. Applied Physics Letters, 72(24), 31663168.

77. Konstantatos, G., Levina, L., Fischer, A., \& Sargent, E. H. (2008). Engineering the temporal response of photoconductive photodetectors via selective introduction of surface trap states. Nano Letters, 8(5), 1446-1450.

78. Ahmadivand, A., Sinha, R., Karabiyik, M., Kaya, S., \& Pala, N. (2016). Fractal aluminum Cayley-trees to design plasmonic ultraviolet photodetectors. SPIE Defense Security, 98362Y.

79. Bie, Y. Q., Liao, Z. M., Zhang, H. Z., Li, G. R., Ye, Y., Zhou, Y. B., Xu, J., Qin, Z. X., Dai, L. and Yu, D. P. (2011). Self-powered, ultrafast, visible-blind UV detection and optical logical operation based on $\mathrm{ZnO} / \mathrm{GaN}$ nanoscale $\mathrm{p}-\mathrm{n}$ junctions. Advanced Materials, 23(5), 649-653. 


\section{CHAPTER 4}

\section{Quantum and Junction Plasmonic Modes}

If the capacitive offset gap spot between closely-coupled nanoparticles fills with a conductive junction, this leads to the direct shuttle of charges across the nanoparticles and accumulation of charges and resulting in the excitation of new plasmonic moment, known as charge transfer plasmons (CTP). The CTP is a highly tunable mode is the direct result of quantum mechanical effect that possesses unique and exotic properties with a broad range of applications for tailoring and building plasmonic sensors, modulators, nanomotors, and artificial molecules [1-5]. The CTP modes are distinguished spectral features, arising at the energy states far from the classical EM multipoles. As an exquisite spectral feature, the CTP modes have been induced successfully using several methods. Four fundamental techniques have been introduced for the excitation of CTP modes using both experimental and numerical studies: 1) direct quantum tunneling [6,7], 2) FowlerNordheim indirect quantum tunneling [8], 3) direct charge transfer in linked nanostructures $[3-5,9,10]$, and 4) conductive sublayer-mediated nanostructures $[11,12]$. In this chapter, we will describe two fundamental principles for the excitation of CTP resonant moments, and the utilized methods to enhance the tunability of CTP modes are discussed in details. Using numerical and experimental studies, we show the transition from capacitive coupling to direct charge transfer in $\mathrm{THz}$ plasmonic assemblies. In addition, to enhance the tunability of optically induced CTP modes, we use optothermally and optoelectronically controllable sublayers beneath a pair of nanoparticles to show how the CTP modes can be tuned along the NIR spectra and the associated devices will be presented and characterized. 


\subsection{Tunneling Plasmonics}

The incident intense beam can be efficiently confined in subwavelength metallic structures due to reduced field penetration through a dense electron sea [13]. Reducing the gap distance between nanoscale particles or cavities down to atomic scales (below 1 nm or a few Angstroms) leads to direct tunneling of optically excited charges and separation of charges, describing by tunneling plasmonic principle in the quantum regime [6]. In terms of theoretical analysis, the physics behind the formation of plasmonic moments in tunneling regime cannot be modeled through hydrodynamic approach for quantum effects [14]. On the other hand, the experimental realization of nanoparticles

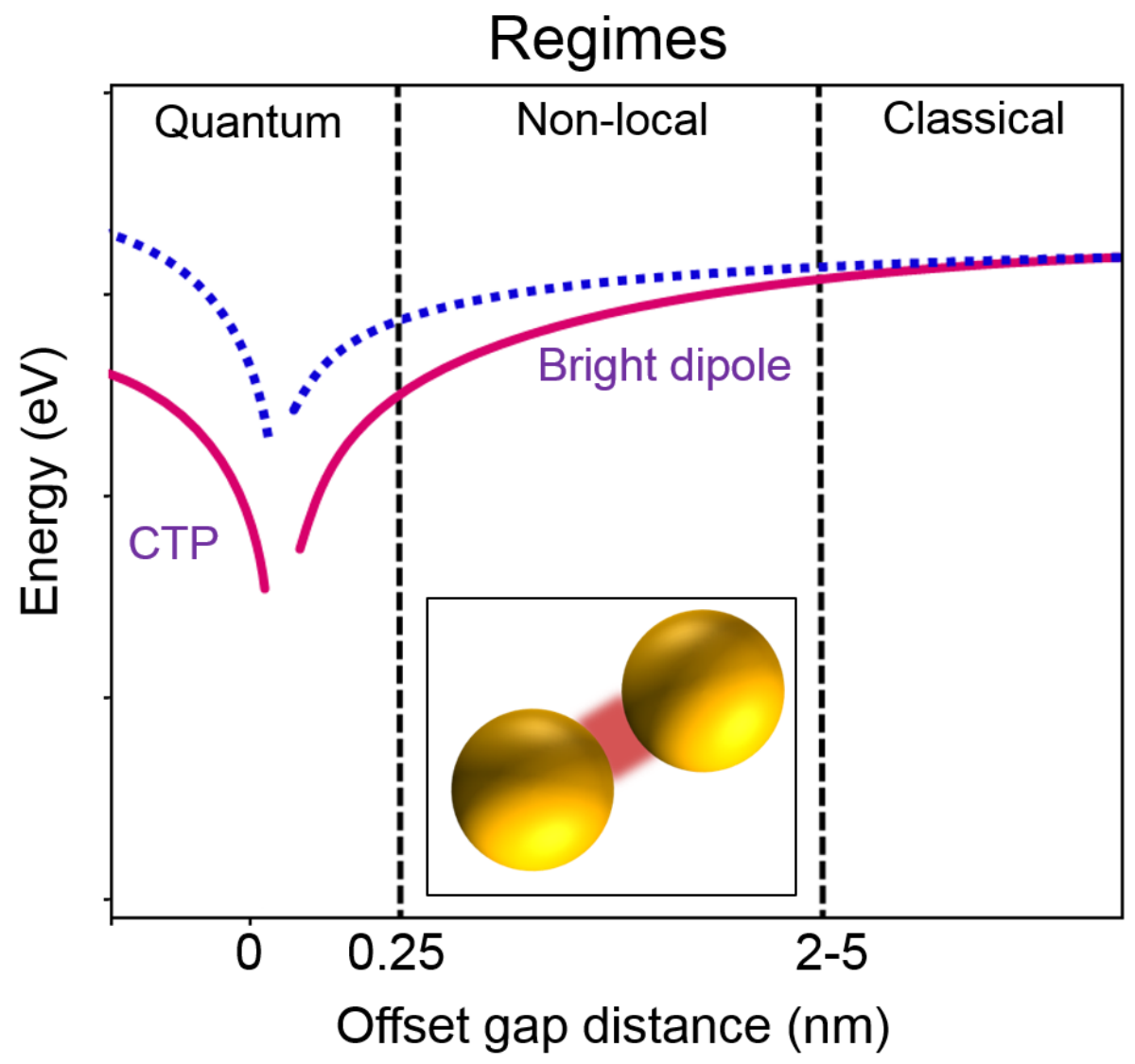

Figure 4.1. General spectral response of a dimer in classical and quantum models. The solid line is for the classical model and the dashed line demonstrating the non-local hydrodynamic model for the quantum model. The inset is a dimer antenna. 
with atomic scale gaps is challenging and reaching the quantum tunneling regime is complex. The first observation of this phenomenon was reported by using two goldnanoparticle-terminated atomic force microscope (AFM) tips with tip-to-tip orientation [6]. It is presumed that the tips apices act as a cavity, supporting plasmonic resonances created through strong coupling between localized plasmons on each tip [15]. On the other hand, as a numerical approach, time-dependent density functional theory (TDDFT) model has been introduced as a numerical tool to study the quantum tunneling principle based on density functional theory with exact reformulation of time-dependent quantum mechanics [6]. In addition quantum corrected model (QCM) has been developed by Esteban et al. [17], which incorporating the electron tunneling effect into the local classical formulism. In contrast to classical calculations, quantum model results for very narrow gaps show pronounced effects of electron tunneling such as a strong reduction of the field enhancement, a continuous transition of modes between the non-touching and contact regimes and the appearance of a CTP mode before the nanoparticles touch [18]. Figure 4.1 illustrates the sketch of two different regimes for plasmon resonance of sphere-dimer in vacuum, identified as a function of the separation offset distance $(d)$. These regimes are demonstrated by the energies of the plasmonic moments estimated by classical hybridization (solid line) and non-local hydrodynamic model for quantum regime (dashed line). As we mentioned formerly, for large gaps in the range of few nanometers, the system is in classical regime and the corresponding plasmonic response can be explained and analyzed by using Maxwell's equations. Conversely, in the nonlocal regime, the actual position of the screening charges strongly depends on the conductivity of the gap and its physical distance. It should be underlined that in the 
plasmon tunneling limit, the AC tunneling current across the atomic opening strongly perturbed the optical response. While this effect becomes more obvious when the conductivity of atomic junction becomes larger than the threshold conductivity of the gap.

In conventional plasmonic systems, the size of the offset gap between proximal nanoparticles is much smaller than the radius of the curvature. In this limit, the local geometry of the nanojunction can be estimated as two flat semi-infinite parallel metallic surfaces that are separated by a dielectric gap with the size of $d$. To compute the electron transmission through the potential barrier between the flat free-electron metal as a function of energy $(\Omega)$ and separation opening $(d)$, one needs to employ a wave packet propagation approach proposed by Zugarranurdi et al [19]. This method is operating based on launching an electron wave packet as a probe from the inside of one of the meals onto the junction. Two virtual detectors that are placed in the asymptotic areas are responsible to collect the transmitted and reflected electron fluxes, required to extract the transmission and reflection profiles. By assuming the incident beam along the $z$-direction, the evolution of the probe electron wave packet can be written using 1D time-dependent Schrödinger equation. In the real plasmonic systems, the properties of the effective medium inside the atomic gap can be extracted using the full atomistic quantum calculations. Accordingly, the plain form of the model potential for the electron interaction with a semi-infinite metal surface can be described by [20]: 


$$
\begin{cases}V_{s}\left(z-z_{i m}\right)=\frac{\exp \left(-\zeta\left[z-z_{i m}\right]\right)-1}{4\left[z-z_{i m}\right]} ; & z>z_{\text {im }} \\ V_{s}\left(z-z_{\text {im }}\right)=-\frac{U_{0}}{A \exp \left(-B\left[z-z_{\text {im }}\right]\right)+1} ; & z \leq z_{\text {im }}\end{cases}
$$

in which $U_{0}, z_{i m}$, and $\zeta$ are parameters for the metallic surfaces with the orientation of <111>. The numerical values for $A$ and $B$ can be determined by requiring continuity of the potential and its derivatives at $z=z_{i m}$. In the jellium model for metallic components, the tunneling barrier determines by the vacuum part of the interface potential, hence, the defined transmission of the junction is strong with respect to the choice of the surface orientations [17-20]. For an electron located on the vacuum side above the surface of the particle ( $z>z_{i m}$ ), the corresponding potential can be determined by the first term of Eq. 4.1 [21]. For the nearly touching regime of metallic nanoparticles, the image potential must be corrected by the insertion of the multiple image term $\left(V_{m i}(z)\right)$ accounting for the cross terms in the screening interactions [20]:

$$
V_{m i}(z)=\frac{1}{4} \sum_{n=1}^{\infty}\left\{\frac{-1}{\left[z-z_{i m}^{(l)}\right]+n Z}+\frac{-1}{\left[z-z_{i m}^{(r)}\right]+n Z}\right\}, \quad z_{i m}^{(l)} \leq z \leq z_{i m}^{(r)}
$$

where the indices $(l)$ and $(r)$ stand for the left and right metallic surface, respectively. For $z$ inside the metals, $z<z_{i m}^{(l)}$ or $z>z_{i m}^{(r)}$, the multiple image term vanishes $V_{m i}(z)=0$. The jellium model potential parameters for various metals such as Au and Ag are quantified and reported in Ref. [17], describes the position of the image plane (zim) and jellium edge $z_{g}$ to the position of the surface atomic layer $z a l$. Accordingly, by taking the distance $l$ between the two surfaces, then: 


$$
l=z_{g}^{(r)}-z_{g}^{(l)}
$$

Finally, the total potential accounting for the long-rage image-potential tail of the electron-surface interaction is specified by [20,21]:

$$
U(z)=V_{s}\left[z-z_{i m}^{(l)}\right]+V_{s}\left[z_{i m}^{(r)}-z\right]+V_{m i}(z)
$$

In the plasmon tunneling calculations, the conductivity of the gap, and the distance between two surfaces in atomic dimensions play key role in defining the total potential and other corresponding parameters. It is shown that there is good agreement between the QCM and TDDFT models in strongly coupled plasmonic nanostructures. The major idea behind the QCM and TDDFT simulations is how the charges behave between metalmetal surfaces with atomic gaps, and also these models allow for demonstrating the possibility of quantum tunneling with fully classical analysis of the optical response and properties of nanoparticle clusters with narrow junctions. The distinguished result of such a tunneling effect is the excitation of CTP resonances. However, the need for advanced and complex fabrication techniques and also complicated quantum mechanical computations have stimulated researches to replace much easier methods to induce CTP resonances with high quality and simpler analysis methods. It should be noted that in the quantum tunneling, due to the limited geometrical tunability and fixed dielectric constant of the gap, hence, the tunability of the induced CTP mode via tunneling of photoinduced charge is extremely limited and can be just control by the intensity of the incident beam [9]. To address these limitations and also to enhance the functionality of CTP-type resonant moments, in continue, we consider one of the novel and highly tunable methods to excite CTP resonances and their applications for developing advanced nanophotonic 

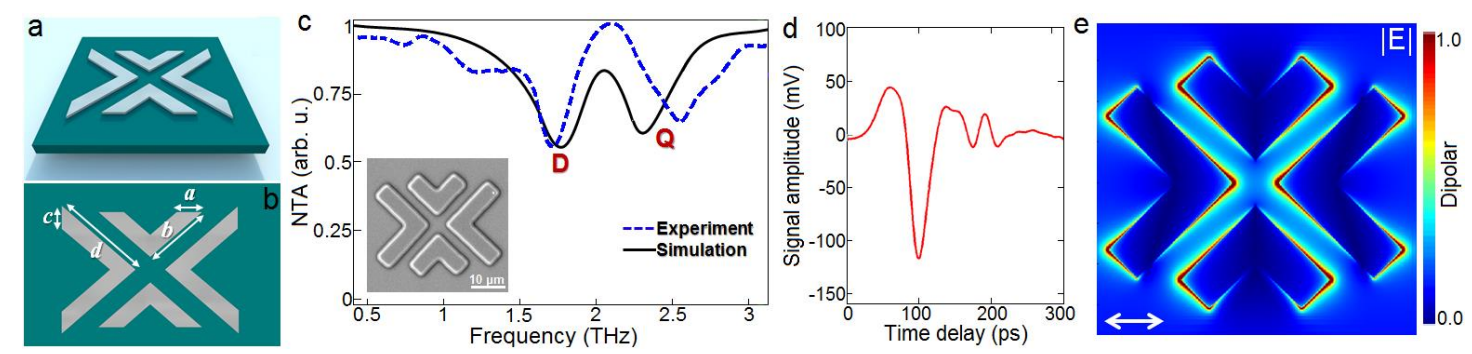

Figure 4.2. a) and b) The schematic and tip view pictures of the assembly, respectively. c) Calculated and measured normalized transmission amplitude (NTA) for the capacitive coupling for the following dimensions: $a / b / c / d=10 / 25 / 10 / 35 \mu \mathrm{m}$. The inset is an SEM image of the plasmonic assembly. d) The applied electro-optical signal amplitude $(\mathrm{mV})$ as a function of time delay (ps). e) E-field map for the asymmetric assembly under longitudinal polarization excitation, respectively.

devices. In the next sections, we present numerical and experimental studies for the excitation of CTP moments in THz plasmonic metasurfaces designed, fabricated and analyzed as part of this dissertation work.

\subsection{Plasmonic Switching Based on Direct Charge Transport}

The presence of a conductive pathway between neighbor nanoparticles leads to the shuttle of optically driven charges across the junction and accumulation of charges, and thereby excitation of CTP resonances [5,9]. It is shown that the AC conductivity of the nanojunction between nanoparticles play fundamental role in the excitation of CTP resonances [22]. As leading studies, Pérez-González et al. [22] and Wen et al. [9] have shown that connecting a pair of adjacent nanoparticles by a conductive link leads to the direct transition of charges across the junction. Evidently, the conductivity of the nanobridge between particles plays a crucial role in controlling the shuttling charges as well as the excitation of CTP. To illustrate the impact of the conductance, the conductivity of bridge can be modeled as a pure conductor with conductivity of $\kappa_{J}$ as follows [22]: 


$$
\varepsilon(\omega)=1+i\left(\frac{4 \pi \kappa_{J}}{\omega}\right)
$$

The corresponding conductance of the junction can be quantified analytically as a function of the geometry of the bridge using:

$$
G=\hat{G}\left(\mathrm{R}_{\mathrm{ext}}, \Delta\right) \kappa_{J}
$$

To show the dependence of the CTP to the bridge quality, one needs to move from the capacitive coupling to the direct charge transfer gradually. To this end, by utilizing both numerical and experimental studies and monitoring the plasmonic response of a four-member quadrumer from capacitive coupling through a spacer to direct shuttle of charges, we showed that the CTP resonances can be efficiently excited at the THz regime [10]. This example provides better understanding of the excitation of CTP resonances simple manner. Here, we used an assembly of V-shaped blocks, and the schematic is shown in Fig.4.2a for the V-shaped assembly (not to scale). The capacitive gap between the resonators was set to $12 \mu \mathrm{m}$. Figure $4.2 \mathrm{~b}$ specifies the geometrical parameters of the plasmonic system. The corresponding geometrical sizes are described in the figure caption. The normalized transmission amplitude for the plasmonic assembly is shown in Fig. 4.2c. The capacitive coupling plays major role in emerging of distinct dips along the curve. The deeper minimum around $1.75 \mathrm{THz}$ correlates with the dipolar resonant mode, while the quadrupole mode appears as a weaker dip around $2.35 \mathrm{THz}$. The inset is the scanning electron microscopy (SEM) image of the fabricated $\mathrm{THz}$ plasmonic assembly. Figure $4.2 \mathrm{~d}$ shows the applied electro-optical signal amplitude as a function of time delay, utilized in the $\mathrm{THz}$ time domain spectrometer (TDS) setup. The comparison with the simulated curves allows us to interpret the charge transfer plasmon resonances. Figure 

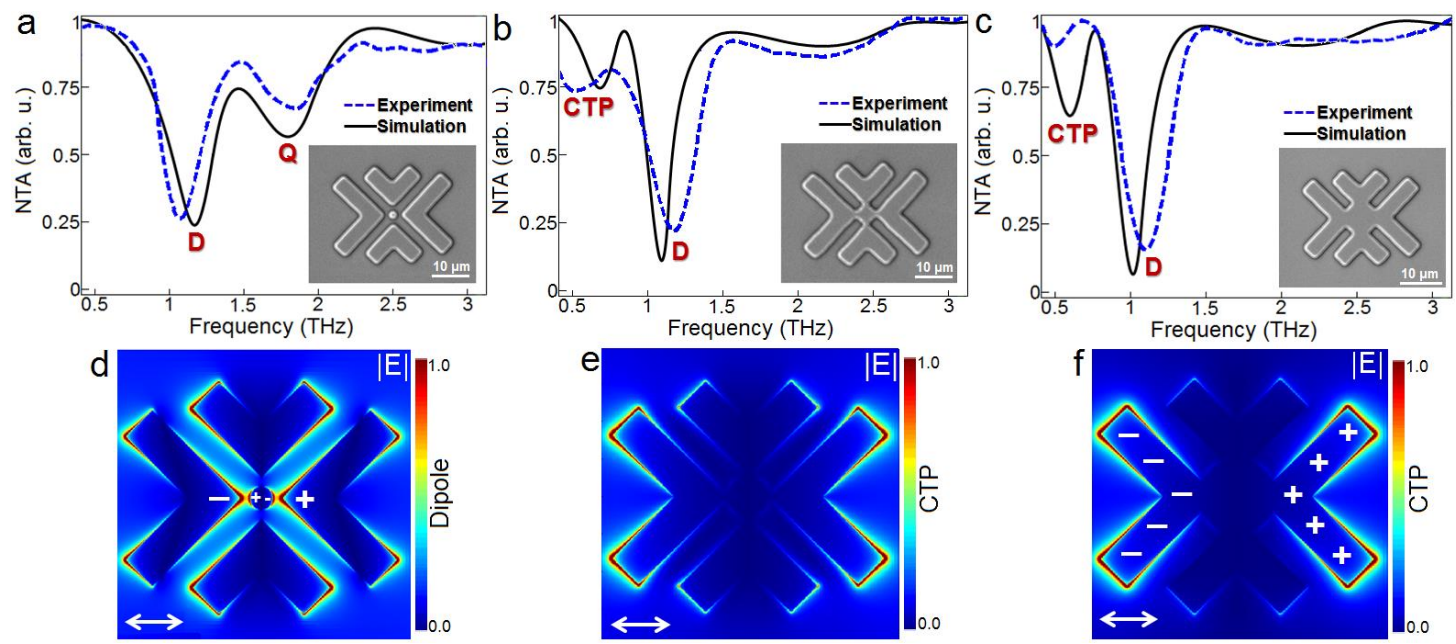

Figure 4.3. a) Characterized and simulated NTA profiles for the asymmetric THz assembly for the presence of (a) a nanodisk between gaps, (b) a touching disk to the VSMBs, and c) an overlapping nanodisk. The insets are the corresponding SEM pictures for nanodisk variations. d), e), and f) The corresponding E-field maps for different studied regimes for the CTP mode. The charge transfer is plotted inside the numerically defined maps.

trates the E-field map for the plasmon resonance excitation and concentration in the junction area of the V-shaped blocks at the frequency of the dipole dip. The charges are mainly concentrated at the tips of the blocks that are parallel to the incident beam polarization. The plasmonic assembly were fabricated by photolithography technique on a high resistivity silicon wafer $(>10 \mathrm{k} \Omega . \mathrm{cm})$ (to provide the required transparency in the $\mathrm{THz}$ domain). Two layers of the positive photoresists (LOR 3B and S1805) with the total thickness of $2.6 \mu \mathrm{m}$ were deposited and patterned. Employing the e-beam evaporation, we then deposited the $750 \mathrm{~nm}$ of Ti layer at the rate of $5 \AA$ /s $(99.99 \%$ purity, pressure $7.6 \times 10^{-7}$ Torr). The lift-off was performed by immersing in remover PG for $120 \mathrm{~min}$ at $70{ }^{\circ} \mathrm{C}$ followed by IPA and DI water rinse. The assembly arrays were fabricated in the area of $1500 \times 1500 \mu \mathrm{m}^{2}$ with the periodicity of $80 \mu \mathrm{m}$. The SEM pictures shown along the manuscript were obtained using the JEOL 7000 tool. The THz characterization was performed using a THz-TDS setup with the beam bandwidth of $10 \mathrm{GHz}$ to $4.5 \mathrm{THz}$ with 
the average power of $\sim 100 \mu \mathrm{W}$. For the numerical modelling, the FDTD method was used with the PML layers as the workplace boundaries for radiation direction ( $z$-axis) and periodic boundaries for $x$ and $y$-axes. A plane wave pulse of 2.6 ps served as an external $\mathrm{THz}$ source. The Ti dielectric function was taken from the empirically defined values by Palik. Reducing the gap distance between V-shaped resonators is a typical strategy to narrow the transmission dips, however, achieving significant dips needs for very small offset gaps. The fabrication of thin three-dimensional microstructures with small gaps is highly challenging.

Our goal is to demonstrate THz CTPs using the direct transfer of charges between Vshaped blocks. To this end, we inserted conductive disks with the same thickness and material as $\mathrm{V}$-shaped resonators with the varying diameters to follow the transition from the capacitive coupling to the direct transfer of charges. First, the disk with the diameter of $7 \mu \mathrm{m}$ was used, while the gap between disk and V-shaped blocks apexes was reduced to $2.5 \mu \mathrm{m}$ on each side. According to the Mie's theory a particle with the size of the considered disk is able to support dipolar modes at $\mathrm{THz}$ resonances [23]. In this regime, the central disk sustains a strong dipolar mode enhancing the quality of the observed transmission lineshapes via the dipole-dipole interaction. Figure 4.3a depicts the effect of this geometrical variation. In the normalized transmission amplitude profile, both dipole and quadrupole dips were enhanced and shifted to the smaller $\mathrm{THz}$ frequencies, while this enhancement is more appreciable for the dipolar dip. The experimental data is in complete agreement with the numerical predictions. By increasing the diameter of the central conductive disk, we provided a path for shuttling of the charges between $\mathrm{V}$ shaped blocks. In this regime, keeping the other geometries the same and increasing the 

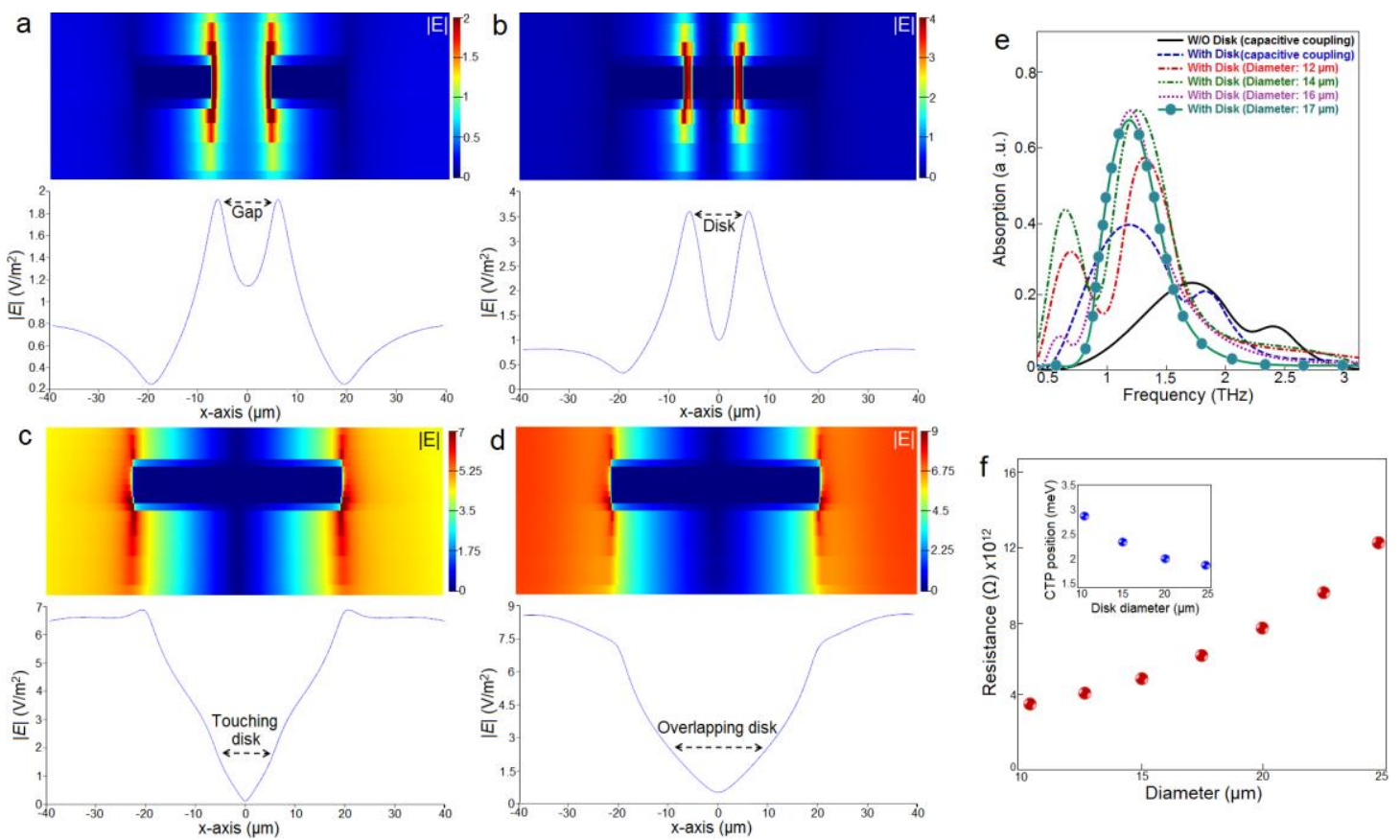

Figure 4.4. a), b), c), and d) The cross-sectional E-field maps and field concentration diagrams for capacitive coupling, presence of a nontouching disk, presence of a touching disk, and presence of an overlapping disk, respectively. e) Comparative absorption profiles for the VSMBs assembly with the absence and presence of intermediate disk with various diameters. f) The resistance variations as a function of the intermediate disk diameter. The inset is the CTP position as a function of conductive disk diameter.

diameter of the conductive disk to $12 \mu \mathrm{m}$ (touch the tips of each block) provided a conductive bridge for the charges. Figure $4.3 \mathrm{~b}$ exhibits the corresponding normalized transmission amplitude spectra for the asymmetric cross design with the central disk touching the blocks apexes. The inset is the SEM image of the fabricated assembly. The major difference compared to the prior case is elimination of the multipolar mode and emerging of the CTP dip around 0.5 THz. Due to the formation of a small pathway between the blocks, we expect direct shuttling of charges in the same way as in the optical nanoscale systems characterized by quantum transition [24]. However, in the examined microstructure for the touching regime, the connected small parts can supports a limited number of transporting charges across the assembly. Increasing the size of the 
central conductive disk to the overlapping regime leads to a sensible shift in the position of the induced resonant modes to the sub-THz spectra, while both dipolar and CTP dips became narrower and deeper. Increasing the size of the overlapping region causes a dramatic damping in the energy of both modes reflecting the electrical properties of the disk that will be discussed in the following. Figure $4.3 \mathrm{c}$ illustrates the normalized transmission amplitude profile for the overlapping regime for both simulation and experimental analysis. The inset is the corresponding SEM picture of the fabricated assembly. The corresponding E-field snapshots illustrate the effect of the conductive disk in the formation of the CTP mode. Figure $4.3 \mathrm{~d}$ presents the structure with a non-touching disk in between, where the plasmons are concentrated at the tips and in the gap areas between the disk and the VSMB apexes. For the touching and overlapping conditions shown in Figs. 4.3e and 4.3f, respectively, the E-field concentration at the middle section of the assembly (disk location) is nearly zero, while the all charges are transported to the left and right sides of the $\mathrm{V}$-shaped blocks parallel to the $\mathrm{THz}$ beam. The charges that are transported to the perpendicular blocks are negligible. This effect is highly distinct for the overlapping regime, and the charges being nearly zero for both the central disk and perpendicular blocks are consistent with the optical charge transfer studies for nanoscale systems [9].

The cross-sectional analysis of the E-field plot along the assembly provides a better understanding for the distribution of the charges across the structure. Figures $4.4 \mathrm{a}-4.4 \mathrm{~d}$ show the E-field maps and diagrams $(|\mathrm{E}|)$ as functions of the assembly position ( $x$-axis) for different regimes. When the intermediate disk is absent, the field localization is concentrated at the apex tips of the V-shaped blocks. Adding the conductive disk to the 
central part and increasing its diameter from non-touching to overlapping condition transfers the localization of the E-field from the central region to the arms tips with the field at the conductive disk becoming nearly zero. The overlapping areas help induce sharper and enhanced plasmonic dipolar and CTP modes. The geometry of the overlapping areas allows to trade-off the charges between the V-shaped blocks of a standalone assembly. This can be described by defining the resistance $(R)$ of the disk as a function of the junction geometry [25]:

$$
R(\Omega)=\frac{2}{\sigma(\omega) t \pi} \ln \left(\frac{2 d}{\delta_{x}}\right)
$$

where $\sigma(\omega)$ is the frequency-dependent conductivity, $t$ is the thickness of structure, $d$ is the length between junctions, and $\delta_{x}$ is the contact width at the junctions. The conductivity is given by $\sigma=n_{T i} e^{2} \tau / m_{e}$ [10], where $n_{T i}$ is the electron density for Ti $\left(4.44 \times 10^{28} \mathrm{~m}^{-3}\right), e$ is the elementary charge, $\tau$ is the mean time between collisions, and $m_{e}$ is the electron mass. Increasing the diameter of the overlapping disk extends the length between junctions, and the corresponding resistance increases accordingly. In this limit, while charges would be able to travel through the pathway, the dissipative losses can be substantial due to the disk resistance.

When incident $\mathrm{THz}$ radiation is resonant with the transmission lineshape, the intense localization of charges across the lossy metallic components causes a dramatic energy dissipation. By increasing the diameter of the intermediate disk, the induced dips shift to the lower energies due to the enhanced dissipative absorption cross-section. This is in contrast with the CTP resonant shift observed in dimers at optical frequencies [9]. We 
compared the absorption spectra for different regimes in Fig. 4.4e, which is showing monotonically growing absorption spectra with increasing the size of the overlapping disk. This can be explained by the resistance increase as a function of the disk diameter (Fig. 4.4f). The inset exhibits the position of the CTP mode as a function of the disk diameter, which is consistent with the experimental results. Obviously, by increasing the size of the middle disk (Diameter $>16 \mu \mathrm{m}$ ), the absorption extreme correlating with the CTP peak is vanished due to strong damping in the corresponding CTP peak, while the absorption at the dipole position is unchanged. Next, Changing the polarization of the $\mathrm{THz}$ radiation to transverse mode $\left(\varphi=90^{\circ}\right)$ resulting with nearly similar plasmonic response with subtle variations in the amplitude of the dipolar dip. For the capacitive coupling, we expected same resonant modes with lower amplitude of dips. For the presence of an overlapping disk, the same CTP peak is expected, while the dipolar dip becomes broader and shallower in comparison to longitudinal case. We also quantified the dephasing time for the induced CTP dip at the THz band using the proposed method based on Fourier-transformation. The Cauchy-Lorentz distribution was used to define the damped harmonic oscillator. Thus, the dephasing time for the induced CTP dip for the presence of overlapping disk is given by: $T_{C T P}=2 \hbar / \mathrm{FWHM}$, where $\hbar$ is the reduced Planck's constant. For the deepest CTP dip at $0.58 \mathrm{THz}$, the dephasing time is estimated as $5.49 \times 10^{-12}$ s $(\sim 5.5 \mathrm{ps})$.

All these studies verify the strong dependency of the charge transfer on the AC conductance and also the corresponding geometrical dimensions of the nanojunction. At first glance, in most of the proposed and analyzed nanostructures, the induced CTP resonances strongly suffer from lack of tunability. As very first studies, to enhance the 

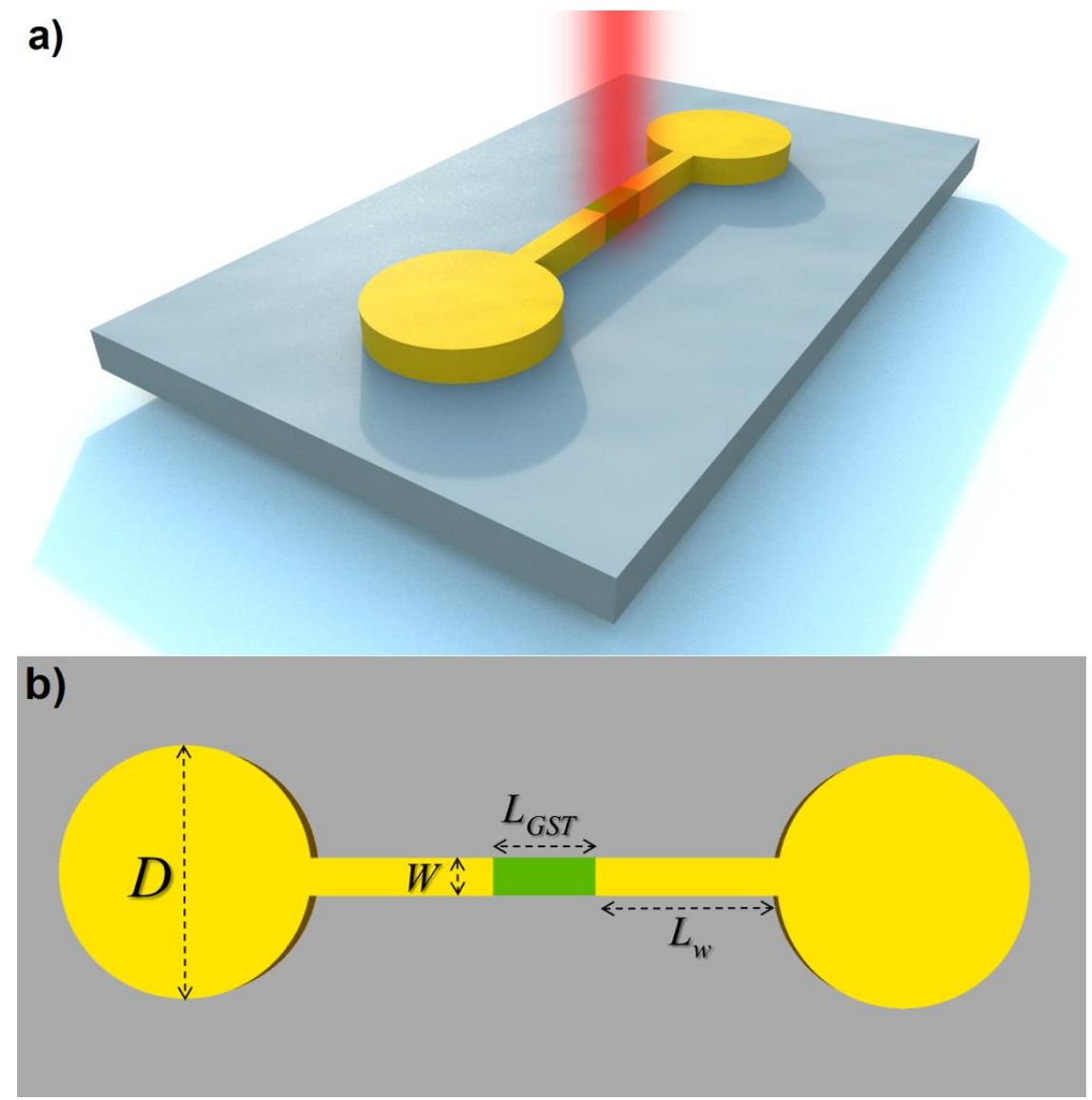

Figure 4.5. a) An artistic rendering of the proposed tunable dimer nanoantenna on a glass substrate. b) A top-view plot of the dimer nanoantenna with corresponding geometrical parameters.

functionality of induced CTP resonances and utilize them in the practical nanophotonic applications, we used optoelectronically and optothermally controllable materials in the blueprints to enhance the tunability. In the next sections, we focus on the functional CTP modes and their applications.

\subsubsection{Optothermally Tunable CTP Modes}

As it is elucidated previously, having active control on the plasmonic properties of a given nanosystem requires the presence of either optoelectronically or optothermally controllable components in the structure. Here, we first focus on the optothermally 
controllable substances to optimize the tunability of CTP moments, and also use them in practical modulation applications for the telecommunication purposes. Recently, phasechange materials (PCM) such as $\mathrm{VO}_{2}$ [26], $\mathrm{Ge}_{2} \mathrm{Sb}_{2} \mathrm{Te}_{5}$ (GST) [27], and AgInSbTe [28] are introduced as promising compounds of which optical properties can be altered by electrical Joule heating or incident radiation. As a specific case, GST or GST225 which is formed by chalcogenide family has been utilized for nonvolatile random access memory (NVRAM) technology due to room-temperature adoptability [29]. In these works, it is demonstrated that GST can keep its state without any external energy source until the next phase change process starts. The small active area filled by GST can be reversibly and quickly toggled among at least two different phases namely a conductive crystalline phase (c-GST) and highly resistive amorphous phase (a-GST) by applying either bias or incident optical pulses. Conventional plasmonic modes such as Fano resonances with tunability by optical stimuli have been observed in metal-GST-metal metamaterials [30].

Figure 4.5a shows an artistic rendering of the proposed nanosystem composed of a couple of plasmonic nanoparticles linked to each other by a metallic nanowire bridge and a GST section located in the middle of the bridge. The intermediate GST layer is in perfect contact with the metallic bridge in both sides in the numerical analysis. The geometrical parameters for each part of the dimer nanoantenna are indicated in Fig. 4.5b. Since our goal is to show the effect of the GST layer on the transition of the excited plasmonic modes, the diameter of the satellite metallic disks are be fixed to $D=200 \mathrm{~nm}$, and the length of the metallic parts $\left(L_{M}\right)$ in the bridge design is varied depending on the size of the GST section. The thickness of the entire system is fixed to $45 \mathrm{~nm}$. Excitation of the CTP mode strongly depends on the geometry and conductivity of the junction 
between plasmonic elements, which allows for transition of charges swiftly. Thus, having active control on the optical properties of this nanobridge would be useful to control the excited CTP mode effectively. This tunability is provided by applying conductivity variations via phase switching in the GST section between the junctions. The wavelength-dependent conductivity of the GST layer can be defined as [31,32]:

$$
\sigma_{G S T}(\lambda)=\frac{c}{2 \lambda}\left(1-\varepsilon_{e f f}(\lambda)\right)
$$

where, $c$ is the velocity of light in a vacuum, $\varepsilon_{e f f}(\lambda)$ is the effective permittivity of GST bridge in the intermediate phases that can be defined by using Lorentz-Lorenz effectivemedium expression at crystallization level [33]:

$$
\frac{\varepsilon_{e f f}(\lambda)-1}{\varepsilon_{e f f}(\lambda)+2}=f_{c}\left(\frac{\varepsilon_{c}(\lambda)-1}{\varepsilon_{c}(\lambda)+2}\right)+f_{a}\left(\frac{\varepsilon_{a}(\lambda)-1}{\varepsilon_{a}(\lambda)+2}\right)
$$

where $f_{i}$ is the volume function of the $i$ th phase as follows:

$$
0 \leq f_{i}=\frac{n_{i}}{\sum_{j} n_{j}} \leq 1
$$

where $n_{j}$ is the density of the $j$ th phase. Transition (switching) between amorphous and crystalline phases is accomplished by formation of localized Joule heating due to illumination with specific power for an adequate duration. The reported critical temperature for this phase change is $\sim 47{ }^{\circ} \mathrm{C}$ [32], which must be achieved via lightmatter interaction by tuning the incident light intensity and absorption coefficient $\left(Q_{a b s}\right)$. It is shown that in the quasi-electrostatic limit, the photothermal heat process for 
compositional nanostructures can be characterized by using multicapacitive cascading approach [33]. This theoretical model contains the enhanced E-field corresponding with the individual parts of a plasmonic nanostructure. The absorbed photothermal heat energy $\left(E_{H}\right)$ in the metallodielectric system can be determined by [34]: $E_{H}=A Q_{a b s} F(r)$, where $A$ is the entire area of the plasmonic dimer, and $F(r)$ represents the optical fluence of the incident gating pulse.

For the crystallization of GST sections of the unit cells, we used an incident continuous wave (Gaussian beam) with the irradiation power of $P_{0}=3.2 \mu \mathrm{W}$, beam fluence of $\phi=60 \mathrm{Jm}^{-2}$, and repetition of $f_{r}=10 \mathrm{KHz}$. Using these settings, for the sample with the distance of " $r$ " form the source, the light fluence defines by [35]: $F(r)=2 P_{0} \exp \left(-2 r / w^{2}\right) / \pi w^{2} f_{r}$, where $w$ is the waist of the Gaussian beam. Finally, the produced thermal power can be calculated as a function of distance and time delay $\left(t_{0}\right)$ : $T(r, t)=\left(A Q_{a} \phi / 1.77 v \tau\right) / \exp \left(-\left(t-t_{0}\right)^{2} / \tau^{2}\right)$ [36], where $\tau$ is the time constant of the irradiation beam. The FDTD method was used to study the proposed plasmonic optical device. Following settings were applied for numerical analysis: The boundaries were surrounded by highly absorptive PML and the incident beam for crystallization was a broadband plane wave with the bandwidth of $400 \mathrm{~nm}-1600 \mathrm{~nm}$, with the irradiation power of $P_{0}=3.2 \mu \mathrm{W}$, beam fluence of $60 \mathrm{Jm}^{-2}$, pulse duration of $2.7 \mathrm{~ns}$, and repetition of $10 \mathrm{KHz}$. The distance from source to the targeted nanostructure was set to $1 \mu \mathrm{m}$. We also defined a light source with the duration of $0.9 \mathrm{~ns}$ and irradiation power of $5.5 \mathrm{~mW}$ to provide the required energy for the change from crystalline to amorphous phase (amorphization process). To provide accurate results, the workplace discretization was set to $0.5 \mathrm{~nm}$ in all of the axes. According to the Courant stability, the simulation time step is 
set to $d t \sim 0.1 \mathrm{fs}$. The permittivity of the glass $\left(\mathrm{SiO}_{2}\right)$ substrate was set to 2.1 according to the Palik constants, while the dielectric function empirically determined by JohnsonChristy was used for the gold plasmonic elements. The corresponding complex permittivity for different phases of the GST were taken from the experimental data reported by Shportko et al. [37].

Using the settings for the Gaussian beam source that are explained above, and also by tuning the dimer geometries, the required thermal heat is produced to switch the GST phase. It should be underlined that a-GST shows low-loss behavior at lower energies (below $E<0.8 \mathrm{eV}$ ) which gives rise to drastically poor absorption coefficient while the cGST shows significant absorption cross-section across this spectra. Figure 4.6a shows the normalized extinction spectra for the dimer system for four different compositional regimes of the bridge. We consider a bridge with the total length of $L_{B}=100 \mathrm{~nm}$ with a GST section with the length of $L_{G S T}=10 \mathrm{~nm}$ and assume that the GST is in amorphous state initially with $0 \%$ crystallization and then switched to full crystallization state (100\%). For the entirely gold bridge without PCM, a classical bright dipolar resonant mode is induced due to capacitive coupling between nanoparticles at $\lambda=0.73 \mu \mathrm{m}$, and a pronounced CTP extreme appeared at the longer wavelengths $(\lambda=2.4 \mu \mathrm{m})$ due to the shuttling of the charges across the dimer through the conductive pathway. By removing a small part of the metallic wire and making an offset gap of $10 \mathrm{~nm}$ (air space) as a capacitive region at the center of the metallic bridge, the same dipolar mode is observed due to coupling of the distant nanodisks, while a significant dipolar peak is also induced due to capacitive coupling between rectangular bridges. It should be noted that the second extreme is more intense due to small gap distance between bridge arms. Insertion of a 
short a-GST section with high resistivity (in the range of $\sim 10^{4} \Omega . \mathrm{cm}$ ) at the center of the bridge causes the more intense dipolar peak to red-shift to the longer wavelengths $(\lambda=2.2$ $\mu \mathrm{m})$. Due to the low-loss behavior of the a-GST at low energies $(k \sim 0)$, the extinction coefficient at the GST interface is negligible, hence, the induced dipolar mode remains intense.

Switching the state of the GST to full crystallized phase (100\% crystallization) results in a substantial decrease in the corresponding resistivity $\left.\left(\sim 10^{-3} \Omega . \mathrm{cm}\right)\right)$ and charge

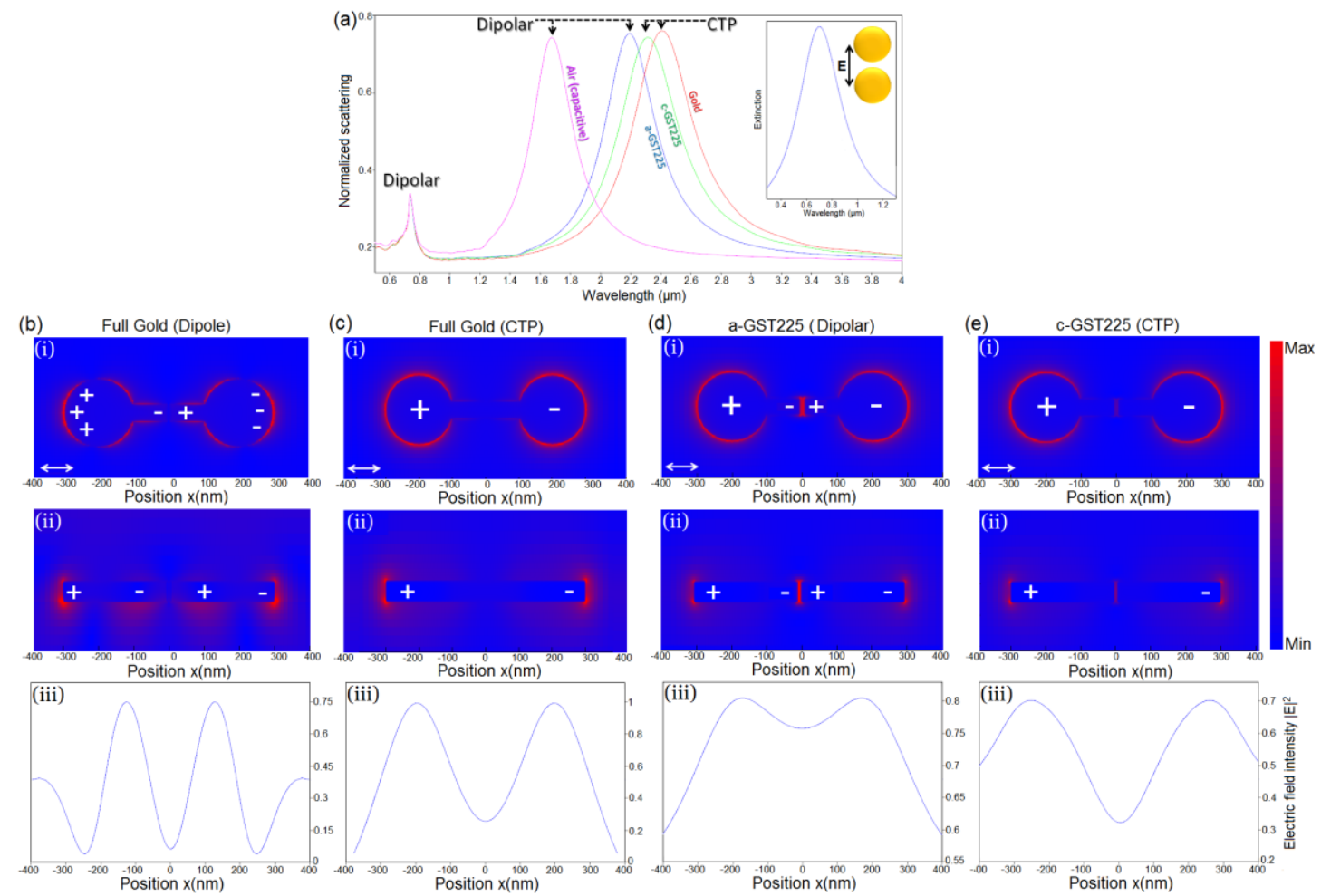

Figure 4.6. a) Normalized extinction spectra of the bridged dimer in GST-mediated, fullmetallic, and air regimes. The inset is the normalized extinction cross-section for the same dimer without conductive junction and plotted for the capacitive coupling regime (the offset gap between nanoparticles is $15 \mathrm{~nm}$ ). b), c) E-field maps across the bridged dimer for dipolar and CTP resonant mode in full metallic regime, respectively, for both (i) top-view and (ii) crosssectional views. d), e) The local E-field distributions correlating with the dipolar and CTP resonant peaks, respectively, for both (i) top-view and (ii) cross-sectional views. (iii) The electric-field intensity diagrams $|E|^{2}$ for the metallodielectric and metallic dimers at the position of CTP and dipolar modes. 
transfer becomes dominant mechanism (negligible capacitive coupling). The result of such a charge transfer is formation of a significant CTP peak at $\lambda=2.3 \mu \mathrm{m}$. Here, the notable red-shift in the position of CTP mode is caused by the absorptive behavior of the c-GST layer at low energies. As shown in the figure, there is $\delta \sim 100 \mathrm{~nm}$ difference between dipolar and CTP modes for different GST phases. Position and amplitude of the leftmost dipolar mode shoulder is particularly independent of the conductive bridge properties for all examined regimes. The inset is the comparative extinction curve for an isolated dimer structure with capacitive coupling showing the induced dipolar peak around $0.7 \mu \mathrm{m}$. Figures $4.6 \mathrm{~b}$ and $4.6 \mathrm{c}$, and subsequent diagrams exhibit the E-field maps (i and ii) and intensities (iii) for the position of dipolar and CTP modes in full gold regime, respectively, revealing the charge distribution difference with the CTP mode. For the presence of GST intermediate layer, the gap distance between nanodisks is set to 15 $\mathrm{nm}$ and the corresponding local electric-field (E-field) distribution across the antenna for three-different regimes are shown in Figs. 4.6d and 4.6e. For the junction between plasmonic nanodisks with a-GST, opposite charges are concentrated in the nanodisks as well as around the dielectric junction (Fig. 4.6d), while a concentration of E-fields is visible across the GST section caused by its resistive behavior, which causes a small damping in the induced dipolar peak. For the c-GST, the E-field is much lower, and the extinction of plasmons is still obvious (Fig. 4.6e), however, the charges can pass through the bridge and the capacitive coupling is eliminated. In contrast, in the entirely gold bridge regime, the charges are transported easily and their concentration around and in the middle of the bridge is invisible compared to the other metallodielectric regimes (Fig. 4.6c). Comparing the calculated E-field intensity profiles for all of the examined regimes 


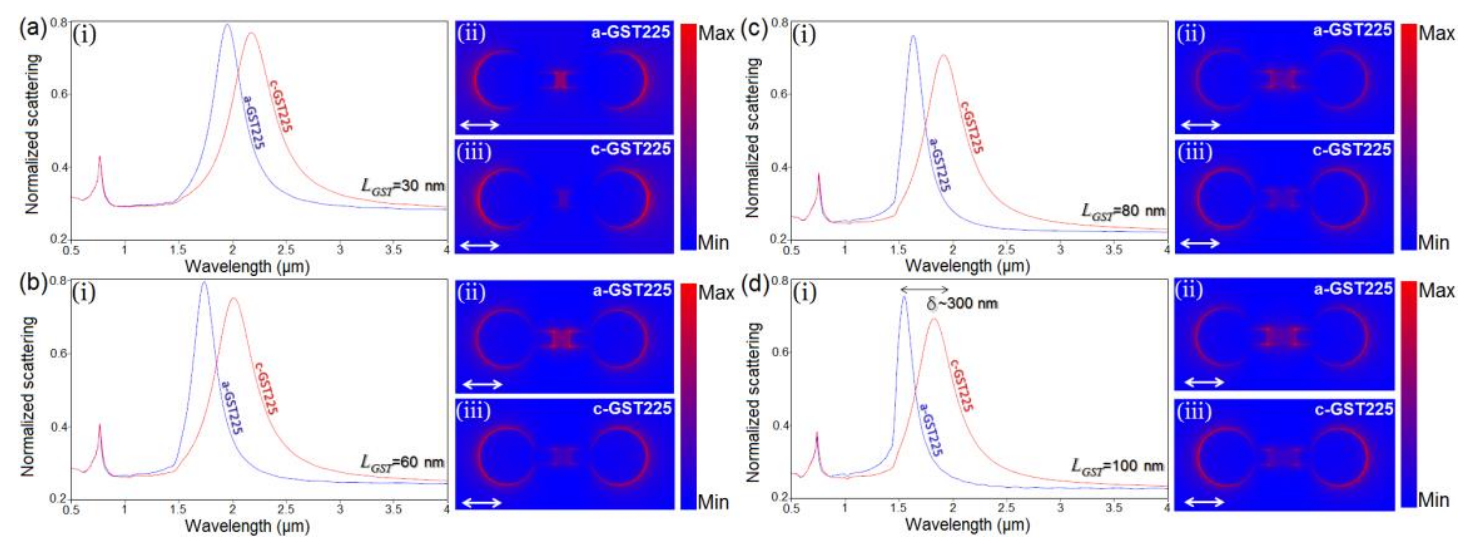

Figure 4.7. a), b), c), and d): (i) Normalized extinction spectra for the GST-mediated bridged dimer, while the $L_{G S T}$ is variant. (ii) and (iii) The corresponding E-field maps for $L_{G S T}$ variations for both amorphous and crystalline phases.

(Fig. 4.6(iii)), with the presence of the dielectric material (GST), the field intensity at the central part of the nanoantenna is reduced, which is consistent with the numerical charge distribution maps.

In continue, we optimize the spectral tunability of the proposed metallodielectric dimer by varying its geometry and monitoring the dipolar and CTP mode response. For the full metallic bridge, it was shown that increasing the length of the nanojunction leads to longer time and lengthier path for travelling of the induced charges across the junction, resulting reduction in the quality of the CTPs significantly [10]. In the presented case, different electrical and optical properties of the amorphous and crystalline phases of GST provides the key advantage for tunability [29]. The resistance contrast for two opposite states of the GST $\left(R_{a} / R_{c}\right)$ is around $\sim 10^{7}$ [38]. By keeping the overall length of the interconnecting nanobridge fixed at $L_{B}=200 \mathrm{~nm}$, and by varying only the length of the GST junction, we shifted the resonant mode to the shorter spectra for both a-GST and cGST as shown in Fig. 4.7. By increasing $L_{G S T}\left(10 \mathrm{~nm} \leq L_{G S T} \leq 100 \mathrm{~nm}\right)$ and reducing the length of the metallic bridges (the entire bridge length is kept fixed), for the a-GST phase, 

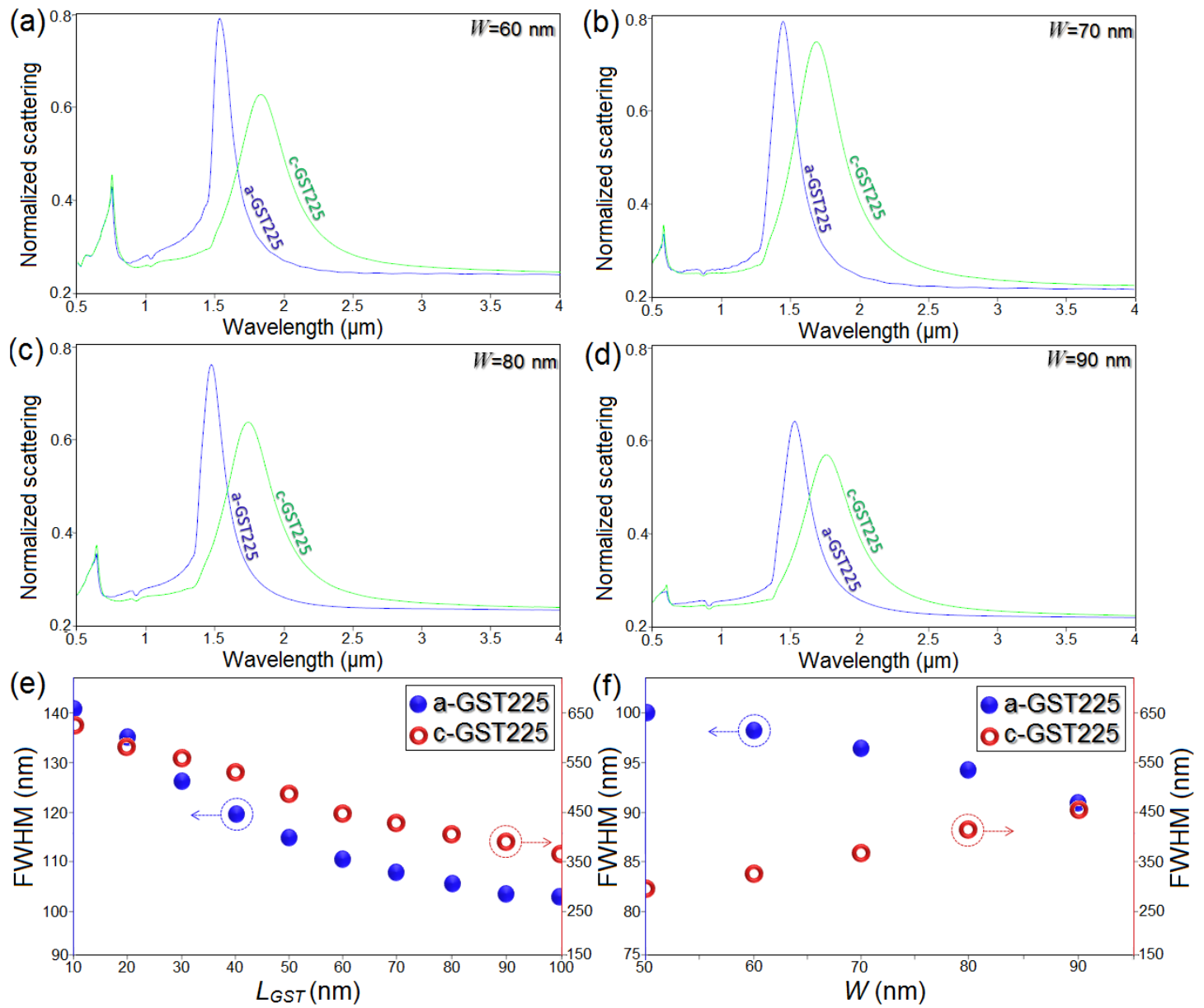

Figure 4.8. a), b), c) and d): Normalized extinction profiles for the GST-mediated bridge dimer antenna with different bridge thickness $(W)$. (e) and (f) The FWHM profiles as a function of $L_{G S T}$ and $W$ for the presence of both a-GST and c-GST layers at the center of bridge.

the dipolar peak is blue-shifted to the shorter wavelengths and became narrower including a small damping in the amplitude of the peak. For the c-GST phase, the CTP extreme is red-shifted for all of the examined sizes, while this shift is approximately three-times larger than the previous analysis $(\delta \sim 300 \mathrm{~nm})$. For instance, for the longest GST length $\left(L_{G S T}=100 \mathrm{~nm}\right)$, a narrow dipolar resonant peak is induced around $\lambda \sim 1.54 \mu \mathrm{m}$ for amorphous state, while for the same geometry and crystallized regime the resonant mode is shifted to $\lambda \sim 1.85 \mu \mathrm{m}$ (as a CTP peak) which is useful for designing NIR optical telecommunication devices. The E-field maps next to the normalized extinction profiles 
(Figs. 4.7(ii) and 4.7(iii)) provide a better view of the effect of the GST length variations on the CTP.

Next, we analyze the effect of further geometrical variations on the resonant extinction peaks as demonstrated in Figs. 4.8a-4.8d. We increased the width of the nanobridge as $W=60 \mathrm{~nm}, 70 \mathrm{~nm}, 80 \mathrm{~nm}$, and $90 \mathrm{~nm}$ for both phases of the GST section. For $W=60 \mathrm{~nm}$, the resonant dipolar and CTP peaks in both states are blue-shifted slightly (Fig. 4.8a), while by increasing the width of the bridge, both peaks show similar shift to the higher energies with the amplitude comparable with earlier investigations (Figs. 4.8b and 4.8c). Here, increasing the widths of the bridge up to $W=80 \mathrm{~nm}$ leads to blue-shift of both resonance peaks to the higher energies. However, continuous increase in the width of the GST and extinction of gold parts of the junction also causes significant damping (decoupling) in the peaks. This decay in the amplitude and energy of CTP mode (for cGST) is significant in Fig. 4.8d. The blue-shift and amplitude damping in both dipolar and CTP modes can be described based on the behavior of the GST material. For the presence of a-GST at the junction, the excited modes in individual metallic arms can couple efficiently leading to formation of strong dipolar mode in the range of 60 $\mathrm{nm}<W<80 \mathrm{~nm}$. In this regime, the dipolar peak slightly blue-shifts to the higher energies. However, by increasing the width of the bridge for $W>90 \mathrm{~nm}$, the energy losses increases drastically leading to an appreciable damping in the amplitude of the dipolar peak. For the presence of c-GST, the charges can transit across the bridge and by increasing the width of the bridge, therefore, more charges can travel to the outermost nanodisks and gives rise to blue-shift in the position of the induced CTP. However, for $W>90 \mathrm{~nm}$, due to inherent lossy behavior of metallic components and also absorptive behavior of 
crystalline PCM for $E>0.8 \mathrm{eV}$, the peak is damped drastically. This decay in both dipolar and CTP is accompanied with a noticeable and progressive damping in the leftmost dipolar shoulder including a giant blue-shift in the position of the dipolar shoulder. Figures $4.8 \mathrm{e}$ and $4.8 \mathrm{f}$ compare the corresponding full wave at half maximum (FWHM) of the induced dipolar and CTP extremes for different phases as a function of GST length and widths. By increasing the length of the GST section, the corresponding FWHM is reduced substantially for both phases of PCM, showing the required narrowness for accurate and fast operations (Fig. 4.8e). For the entire bridge width variations, the corresponding FWHM increased (decreased) with the increasing width for c-GST (aGST) state. These opposing trends can be attributed to the different loss mechanism of the dipolar and CTP modes as explained above. Possessing sharp and narrow peak with small FWHM would help to design high-precision plasmonic devices [39]. However, the obtained narrowness for the wider bridge is accompanied with dramatic damping in the induced dipolar and CTP extremes.

For conventional all-optical and electro-optical plasmonic switches that are tailored to operate at the NIR, low cross-talk and field leakage, and fast switching are the fundamental requirements of high performance. Sharpness and position of the induced dipolar and CTP modes play key roles in determining the suitability of the proposed nanoplatform for optical switching. Our foregoing analysis show that the nanostructure with the geometry of $L_{G S T}=100 \mathrm{~nm}, W=50 \mathrm{~nm}, T=45 \mathrm{~nm}$, and $D=200 \mathrm{~nm}$ is the best candidate for switching applications. Providing approximately $\delta \sim 300 \mathrm{~nm}$ shift for the resonance peak between two different phases (Fig. 4.8d) and fast switching from amorphous to the full crystallization state (requiring a few nanoseconds) as well as 


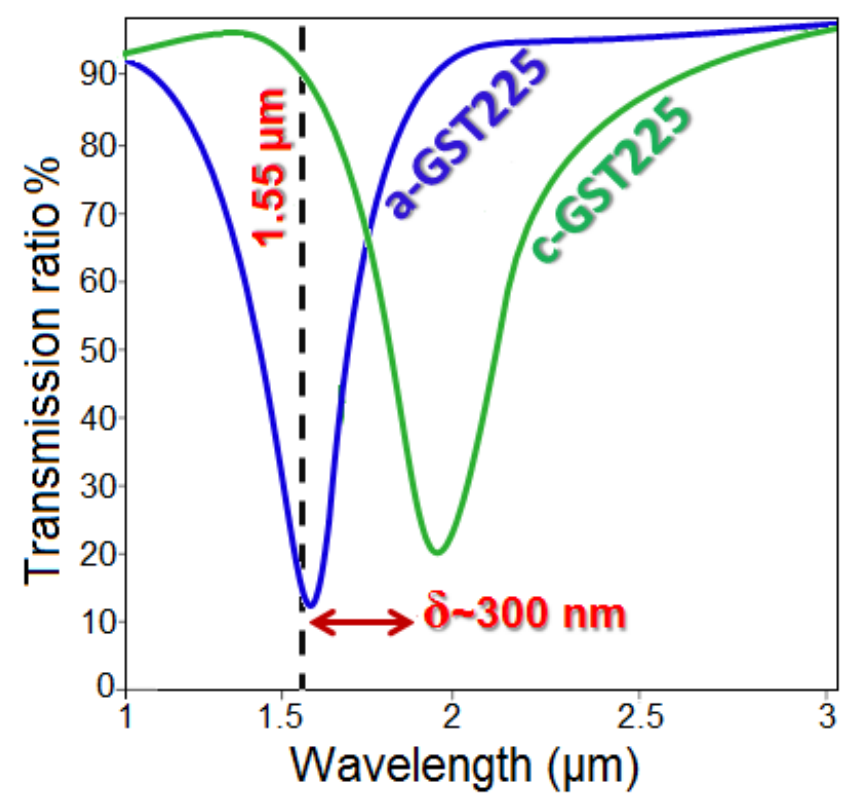

Figure 4.9. The transmission ratio of the GST-mediated metallodielectric switch in OFF (amorphous) and ON (crystalline) states.

switching back to amorphization (requiring hundreds of femtoseconds) [38], these structures could be used for designing fast and efficient plasmonic photonic switches. We demonstrate the switching performance of the studied metallodielectric nanostructure by adjusting the position of the GST-mediated resonant peak centered at $1.55 \mu \mathrm{m}$ and analyzing the transmission ratio, as shown in Fig. 4.9. First we assume that the GST section of the bridge is in amorphous phase (OFF state of the switch). Then, by applying a high-power gating pulse signal [39], crystallization process is started and the resonance wavelength shifts to the lower energies (ON state of the switch). As it was mentioned earlier, in order to reverse the switching process from ON to OFF state, a gating beam with higher power and shorter duration must be applied (see the Supplementary Information). According to the transmission ratio profile, the modulation depth across the telecommunication band $(\lambda=1.55 \mu \mathrm{m})$ is around $88 \%$. As a specific case, for $\lambda=1.55 \mu \mathrm{m}$, the corresponding insertion loss (IL) for switching from OFF to ON state is calculated 
based on the monitored power $\left(P_{m}\right)$ and the incident $\left(P_{i}\right)$ by: $I L=-10 \log _{10}\left(P_{i} / P_{m}\right)$ [3], which yields $\sim 4.5 \mathrm{~dB}$ for $L_{G S T}=100 \mathrm{~nm}$.

Here, using full electromagnetic wave calculations, we have shown that the GST section in amorphous phase hinders direct transfer of charges across the nanobridge and acts as a capacitive region resulting a distinguished dipolar extinction peak at the global telecom wavelength $(\lambda=1.55 \mu \mathrm{m})$, which constitutes the OFF state of the switch. By applying a high power gating pulse to produce the required photothermal heat energy, aGST switches to the crystalline orientation (c-GST) and attains low resistivity at the operating domain, leading to a CTP peak at the lower energies $(\delta \sim 300 \mathrm{~nm}$ apart from the dipolar peak for the a-GST), corresponding to the ON state. Fast and reversible switching performance of the proposed optothermally functional metallodielectric nanostructure could be used for designing efficient all-optical and optoelectronic devices for advanced telecommunication applications.

\subsubsection{Optoelectronically Tunable CTP Modes}

In previous section, we examine the possibility of tuning the induced CTP resonances using optothermally functional substances in the design of the structure. However, switching between amorphous and crystalline phases of a nonvolatile material needs for applying Joule heating at high temperatures $\left(\geq 477{ }^{\circ} \mathrm{C}\right)$, and in some cases, the nanophotonic components in a given device are highly sensitive to high temperature variations. Hence, using tunable materials with electrical-tunability helps to develop temperature-independent nanophotonic devices. In continue, using an analogous strategy to the previously analyzed dimer structure, we induce CTP resonances by replacing the functional junction by an optoelectronically controllable 2D carbon layer, known as 
graphene [40]. Graphene has a wide range of usage in advanced nanophotonic technology and has been acknowledged as a promising substance for plasmonic applications [41].

Recently, Frenzel et al. [42] have verified that the photoconductivity of a monolayer graphene sheet can be tuned efficiently by applying bias via controlling the generated carrier density. Such a feature was achieved by modelling the electronic properties of graphene in terms of massless Dirac fermions [41,42]. In addition, graphene has been successfully demonstrated as a reliable substance for plasmonic purposes due to its substantial optical power absorption along a wide range of spectrum [43], and also its semi-metallic behavior with an optical conductivity as a function of quantum conductance as [43]: $\sigma=\pi e^{2} / 2 h$, where $e$ is the elementary charge and $h$ is the Planck's constant. Similar to the nanoplasmonic components, the spectral response of graphene single-monolayer can be understood by Drude absorption model for a wide range of carrier densities [44]. This temperature-independent model for free carrier photoconductivity with parabolic dispersion in a $2 \mathrm{D}$ monolayer can be written as a function of frequency [40]: $\sigma(\omega)=n e^{2} / m(\Gamma-i \omega)$, where $m$ is the electron mass and $\Gamma$ is the transport scattering rate. It should be underlined that in the presented work, graphene monolayer with local conductivity is presented with the ability to transfer charges with lower decay rate and higher lifetime of charges [42].

All these features and advantages stimulated us to utilize graphene for the transition of charges and induce ultrastrong plasmonic moments such as CTP modes for real applications such as switching. To this end, we report a systematic study of a pair of metallic nanoparticles connected with a gate tunable graphene sublayer to control the transfer of photoinduced electrons across the atomic junction effectively via tuning the 
corresponding carrier density. Using full wave electromagnetic numerical including FDTD and FE methods, we show that the optically excited charges supported by proximal metallic nanoparticles can be tuned and guided by electrically varying the conductivity of the graphene sublayer. The achieved results verified that at the semiconducting regime of graphene capacitive coupling between the dipolar modes on the nanodisks becomes dominant and leads to formation of a distinguished dipolar peak of extinction in the visible region. Then, by applying gate bias to the system, graphene sublayer acts as a semi-metallic conductive layer, in which a new tunable peak appears at the NIR spectra, correlating with the CTP mode in addition to the dipolar peak in the visible domain. Both longer lifetime and better confinement of the optically driven plasmons in $n$-doped graphene layer lead to efficient manipulation of charges in the system by varying the doping level through electrostatic gating approach. Using the interplay between photoexcited charges at both semiconducting and semi-metallic regimes, we adjusted the peak of the CTP mode at the global telecommunication wavelength $(\lambda=1550 \mathrm{~nm})$ to develop a tunable and fast graphene-plasmonic nanoswitch.
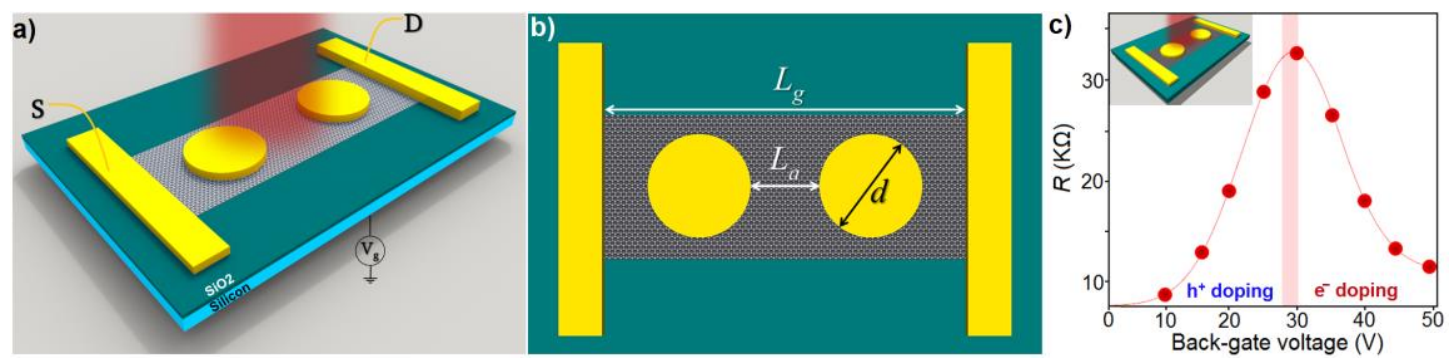

Figure 4.10. a) An artistic rendering of the proposed device composed of gold nanodisks and graphene monolayer. b) A top-view of the device showing the corresponding geometrical parameters. c) Electrical transport characteristic curves as resistance variations $(R)$ as a function of back-gate voltage $(\mathrm{Vg})$ for the proposed graphene-plasmonic platform. The diameter of nanodisks is $d=125 \mathrm{~nm}$ and the offset space between them is $L_{a}=100 \mathrm{~nm}$ while the thickness is $t=45 \mathrm{~nm}$ for both. The distance between source-drain electrodes is $L_{g}=500 \mathrm{~nm}$ and the width of graphene sublayer is $W=160 \mathrm{~nm}$. 
Figures $4.10 \mathrm{a}$ and $4.10 \mathrm{~b}$ illustrate the 3D schematic and top-view image, respectively (not to scale), of the proposed plasmonic switching device composed of a pair of gold nanodisks connected with a single-layered graphene sublayer with back-gated voltage $\left(V_{g}\right)$ as well as source and drain contacts. We used the experimentally dielectric function determined by Johnson-Christy for the gold nanoparticles and relative permittivity of $\sim 2.1$ for the glass $\left(\mathrm{SiO}_{2}\right)$ substrate, measured by Palik. It should be noted that the graphene monolayer is in ohmic contact with the source and drain electrodes in both sides. It should be underlined that the thickness of the graphene monolayer is set to 0.35 $\mathrm{nm}$ and for simplicity the thickness of the plasmonic nanodimer is set to $45 \mathrm{~nm}$ due to having negligible influence on the CTP intensity.

For the absence of gold nanodisks, the graphene monolayer with carrier density close to the neutrality point acts similar to the conventional semiconductors, and we expect generation of electron-hole pairs under light exposure and significant absorption of incident EM wave at this point [45-47]. Conversely, when the charge density in graphene is high, variations in the charge distribution are close to the Fermi level [42]. In this regime, graphene sheet behaves as a conductive substance similar to the metals. However, in the presence of metallic nanoparticles in direct contact with the graphene layer, injection of hot-electrons into the graphene layer plays fundamental role in determining the spectral and electronic response of the system. In the dimer nanoantenna case, the photoinduced hot-electrons generated in the metallic disks are injected into the graphene sheet, resulting in $n$-type doped graphene monolayer, possessing minor role in the doping type. As a major and critical parameter, the doping concentration of graphene can be electrically tuned allowing for switching between semiconductor and semi- 
metallic regimes via the control over its photoconductivity. Figure 4.10c exhibits the resistance $(R)$ variations for the plasmonic dimer nanoassembly on the graphene sublayer as a function of back-gate voltage under incident beam illumination. This characteristic profile indicates the Dirac point for graphene-plasmonic regime. In the grapheneplasmonic limit, the Dirac point is reached at the gate voltage around $\sim 28 \mathrm{~V}$, while the applied source-drain $\left(V_{D S}\right)$ bias in both analyses was set to $1 \mathrm{mV}$ [48]. Using this approach, the carrier concentration qualitatively can be tuned around $\sim 2 \times 10^{13} \mathrm{~cm}^{-2}$. The shift in the Dirac point for the graphene-plasmonic case can be explained by considering the variations in the graphene work function due to direct contact with gold nanoparticles. Comparing the graphene sheet without and with contact with metallic structures, for the latter case, there is a steady inclination of the Dirac energy level along the horizontal direction close to the contact edge due to work function difference between illuminated graphene sheet and the 2D layer beneath the metallic nanoparticles [49].

Figures $4.11 \mathrm{a}$ and $4.11 \mathrm{~b}$ evaluate the extinction spectra for the absence and presence of plasmonic dimer antenna on the graphene monolayer. The electromagnetic response of the nanosystem is obtained for the $n$-doped graphene with the Fermi energy of $E_{F}=0.61$ $\mathrm{eV}$, taken from the experimental report by Fang et al. [50] for graphene disks and rings in hybridized regime $\left(E_{F}=\hbar v_{F}(\pi n)^{1 / 2}\right.$, where, $v_{F}=10^{6} \mathrm{~m} / \mathrm{s}$, and $n$ is the charge carrier density) [41]. Thus for the applied gate bias and incident beam, highly-doped graphene sheet shows substantially conductive behavior and a distinct dipolar peak appears at $E=0.44$ $\mathrm{eV}$, consistent with atomistic analysis achieved by random-phase approximation (RPA) for graphene plasmonics in previous studies [51]. The inset shows the local E-field map for the dipolar plasmon resonance excitation in a graphene layer. On the other hand, for 

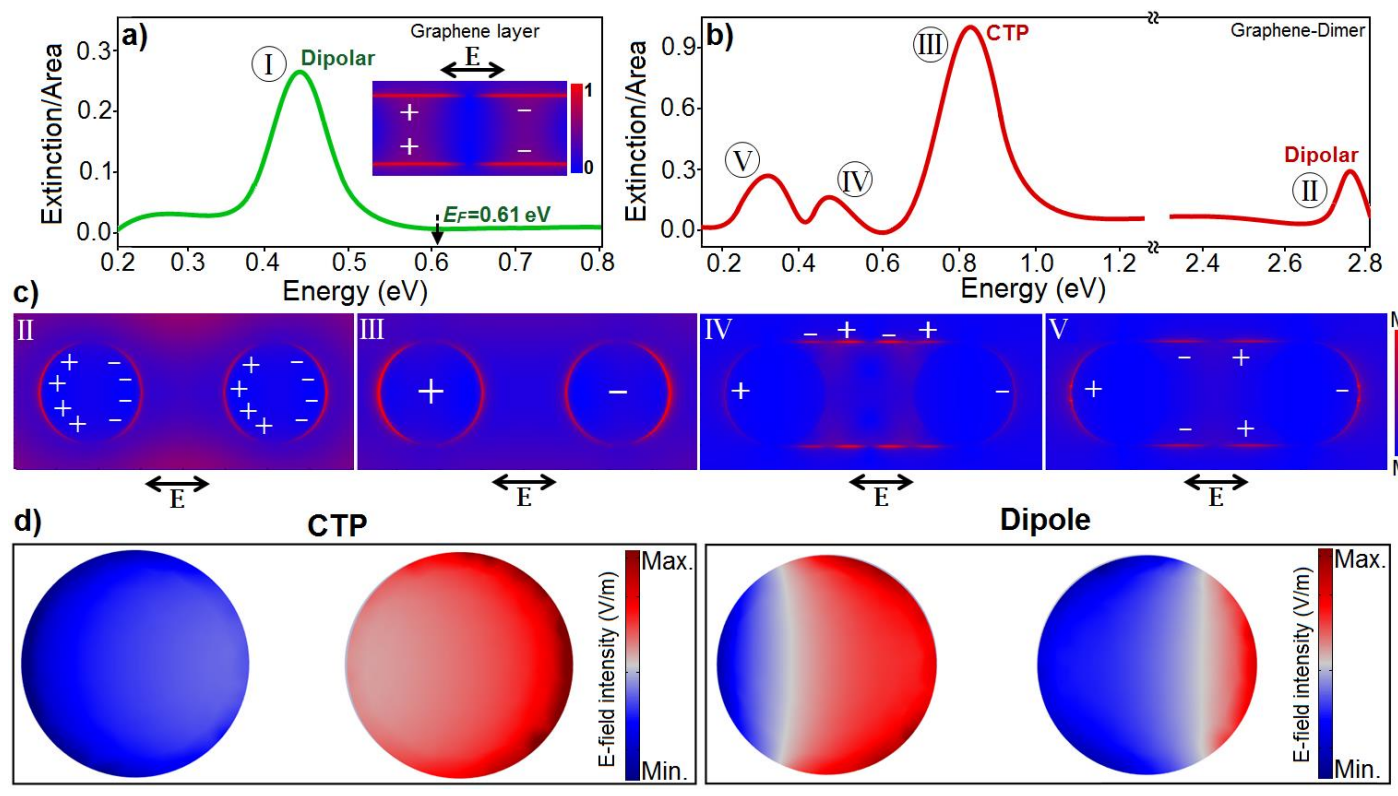

Figure 4.11. a) Extinction cross-section of a highly-doped to $E_{F}=0.61 \mathrm{eV}$ (n-type) graphene nanoribbon under optical excitation at room-temperature $T=300 \mathrm{~K}$. The inset is the E-field intensity map for the dipolar mode. b) Extinction spectra of plasmonic nanodimer antenna on the highly doped graphene nanoribbon bridge, supporting dipolar (II), CTP (III), quadrupolar (IV), and dipolar (V) moments. c) The local $|E|$-field intensity snapshots for the plasmon resonance excitation and distribution across the device for dipole (II) and CTP (III) modes. d) The charge density plots for the CTP spectral feature and dipolar modes.

the presence of nanodisks in direct contact with highly-doped graphene sublayer, we observed formation of distinguished resonant peaks at $E=2.78 \mathrm{eV}$ and $0.81 \mathrm{eV}$, correlating with the dipolar and CTP modes, respectively. The large gap area between proximal nanodisks prevents strong near-field hybridization of plasmons. Here graphene layer acts as a conductive pathway to transfer the charges between the neighbor nanodisks, therefore, opposite charges accumulate at both sides, giving rise to formation of a CTP mode at lower energies. The mechanism of the transport of photoexcited charges can be better understood by analyzing the behavior of graphene at high doping $\left(E_{F}>\omega\right)$ [52]. It is well-accepted that using Drude model, homogenous graphene with plasmonic properties supports propagation of photoinduced electrons with the wave vector of [52]: 


$$
k_{s p} \approx\left(\frac{\hbar^{2}}{e^{2} E_{F}}\right)(\omega(\varepsilon+1)(\omega+i / \tau))
$$

where $\tau$ is the finite relaxation time, and $\varepsilon$ is the relative permittivity. Therefore, due to large photoconductivity of graphene, we expect longer relaxation time compared to noble metals and lower dissipative losses similar to the graphene nanoribbon waveguides with plasmonic properties. In other words, graphene monolayer underneath the metallic particles acts as the charge transfer channel between the metallic $(\mathrm{Au})$ and oxide $\left(\mathrm{SiO}_{2}\right)$ interfaces, has been previously confirmed and measured by Kelvin probe force microscopy [53]. On the other hand, two distinct resonant peaks are induced at $0.43 \mathrm{eV}$ and $0.31 \mathrm{eV}$ correlating with the quadrupolar and dipolar modes supported by conductive graphene sublayer junction between nanodisks. Here, the quadrupolar mode appeared due to intense doping of graphene monolayer simultaneously by the gate bias and injected electrons to the graphene sheet, enhancing the plasmonic properties of the layer. Moreover, variations in the doping properties of graphene lead to a red-shift in the position of the dipolar peak to the lower energies. The charge distribution maps for the graphene plasmonic regime at different modes are illustrated in Fig. 4.11c. Figure 4.11d shows the local E-field intensity for the charge distribution across the dimer for both CTP and dipole modes. These results strongly confirm the claim that at the CTP position the negative and positive charges are concentrated at the opposite sides due to direct transport of charges through the channel, while in the dipolar mode energy, the capacitive coupling becomes dominant due to weak interference of energetic dipolar modes from each nanodisk. 
Here, the optically excited CTP can be simply tuned at the desired energy or wavelength by varying the conductivity of the atomic sublayer. To this end, we carried out specific geometrical variations for the analyzed plasmonic device. Figures $4.12 \mathrm{a}$ and $4.12 \mathrm{~b}$ illustrate the behavior of both dipolar and CTP modes supported by the metallic nanodisks. One should note that we neglected the behavior of dipolar and multipolar modes at the low energies supported with graphene sheet and the Fermi level is fixed to

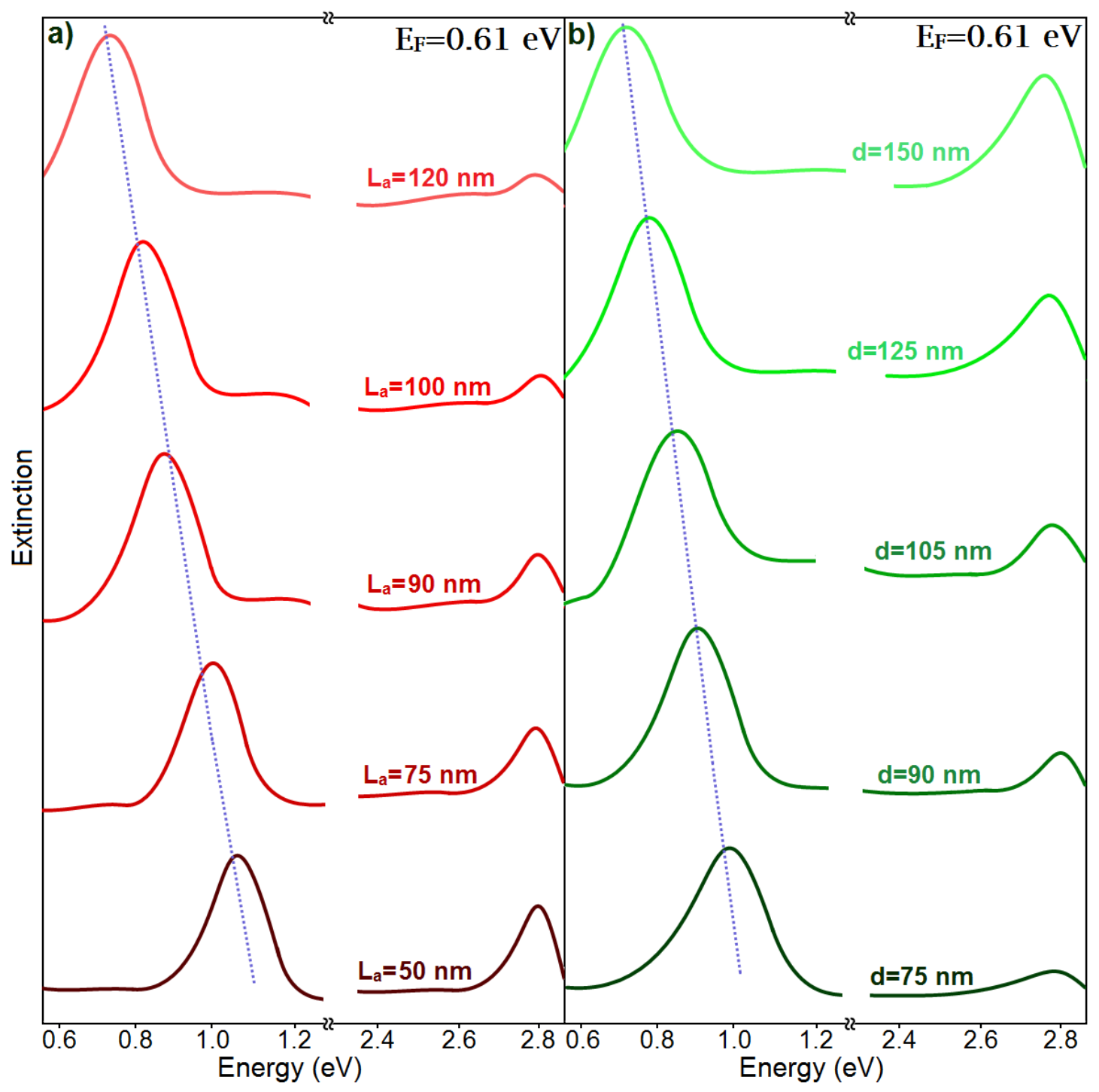

Figure 4.12. a) Normalized extinction spectra for interparticle distance (50 nm $\leq L_{a} \leq 120 \mathrm{~nm}$ ) and b) nanodisks diameters $(75 \mathrm{~nm} \leq d \leq 150 \mathrm{~nm})$ variations, respectively. The doping of graphene is n-type Fermi level energy if fixed to $E_{F}=0.61 \mathrm{eV}$. 
$E_{F}=0.61 \mathrm{eV}$. As shown in Fig. 4.12a, increasing the edge-to-edge distance $\left(L_{a}\right)$ between nanoparticles from $50 \mathrm{~nm}$ to $120 \mathrm{~nm}$ gives rise to dramatic red-shift in the position of CTP mode to the lower energies, while the diameter of the disks is fixed to $d=125 \mathrm{~nm}$. This can be better understood by analyzing the conductance of the graphene junction. It is shown that increasing the length of the conductive bridge between neighbor nanoparticles causes increase in electron travel time between the nanoparticles as well as dramatic decay of plasmons due to longer travel area [3]. Therefore, for the highly-doped $n$-type graphene layer, the frequency-dependent conductance can be written as: $G(\omega)=\sigma(\omega) W T / L_{a}$, where $W, T$, and $L_{a}$ are the width (fixed to $160 \mathrm{~nm}$ ), thickness (here set and fixed to $0.35 \mathrm{~nm}$ ), and the length of the graphene layer, respectively. The frequency-

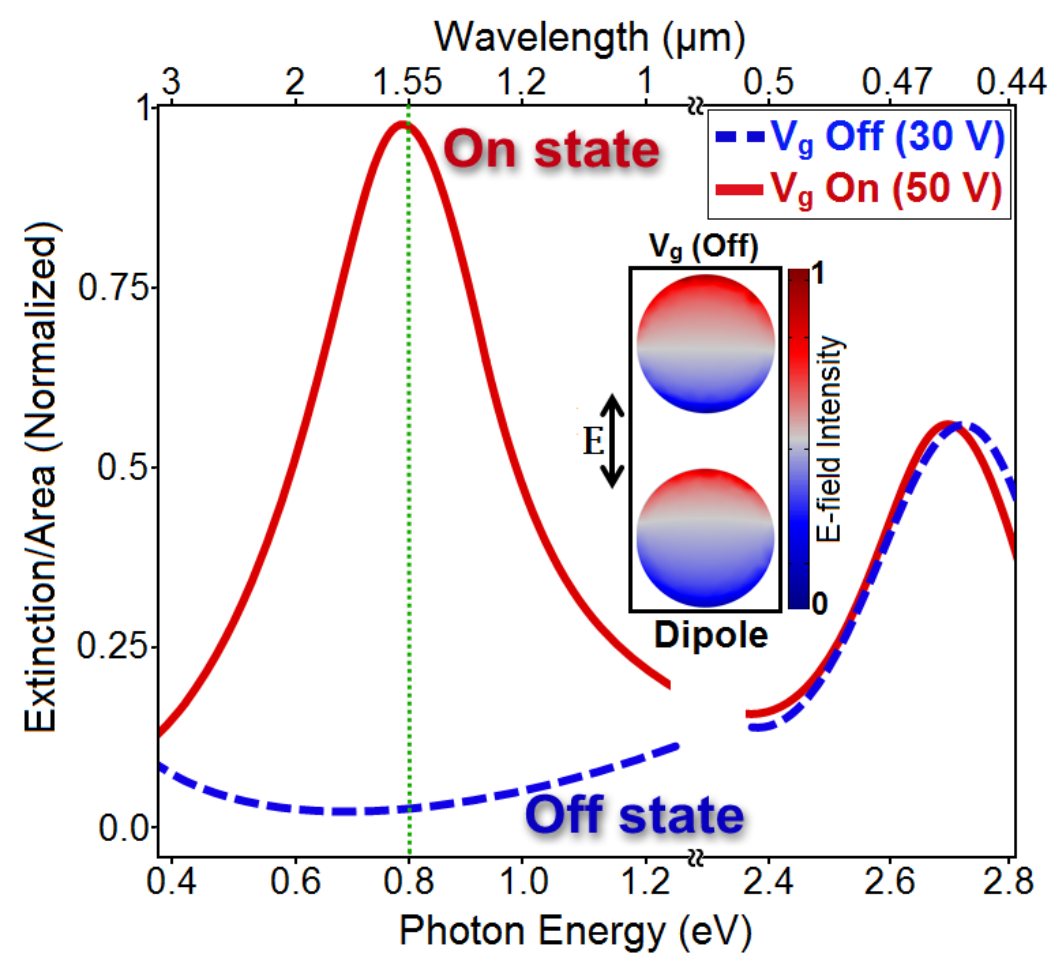

Figure 4.13. Normalized extinction spectra for the "On" and "Off" states of the grapheneplasmonic nanoswitch in the presence and absence of back-gate voltage. The critical geometrical parameters are: $L_{a}=100 \mathrm{~nm}, d=120 \mathrm{~nm}$. The inset is the local E-field map for the dipolar resonance, when the graphene sublayer is in semiconducting regime. 
dependent conductivity of an atomic graphene bridge composed of intraband (Drude model response) and interband transitions (lossy part) has been reported using RPA as below [52,53]:

$$
\sigma(\omega)=\frac{e^{2} E_{F}}{\pi \hbar^{2}}\left(\frac{i}{\omega+i / \tau}\right)+\frac{e^{2}}{4 \hbar}\left(\theta\left(\hbar \omega-2 E_{F}\right)+\frac{i}{\pi}\left|\frac{\hbar \omega-2 E_{F}}{\hbar \omega+2 E_{F}}\right|\right)
$$

Consequently, by increasing the length of the pathway, the conductance reduces slightly and delays the shuttle of the photoexcited charges. Nevertheless, it is noteworthy that due to the substantial conductivity of highly doped graphene sheet, we do not expect dramatic shift in the position of CTP peak to the MIR as it happens in dissipative metallic and lossy junctions. On the other hand, increasing the space between proximal disks causes drastic decay in the amplitude of the dipolar peak at high energies. Figure $4.12 b$ represents the spectral response of the analyzed device for variations in the diameter $(d)$ of the nanodisks. Keeping the dimer system symmetric, we homogenously increased the diameter from $75 \mathrm{~nm}$ to $150 \mathrm{~nm}$, while the edge-to-edge gap is fixed to $L_{a}=100 \mathrm{~nm}$. In this limit, by increasing the size of nanodisks, the CTP extreme red-shifts to the lower energies due to simultaneous increases in both charge separation distance and electron travel time across the structure. The dipolar peak at high energies significantly enhanced by increasing the size of disks due to stronger dipolar interference. 


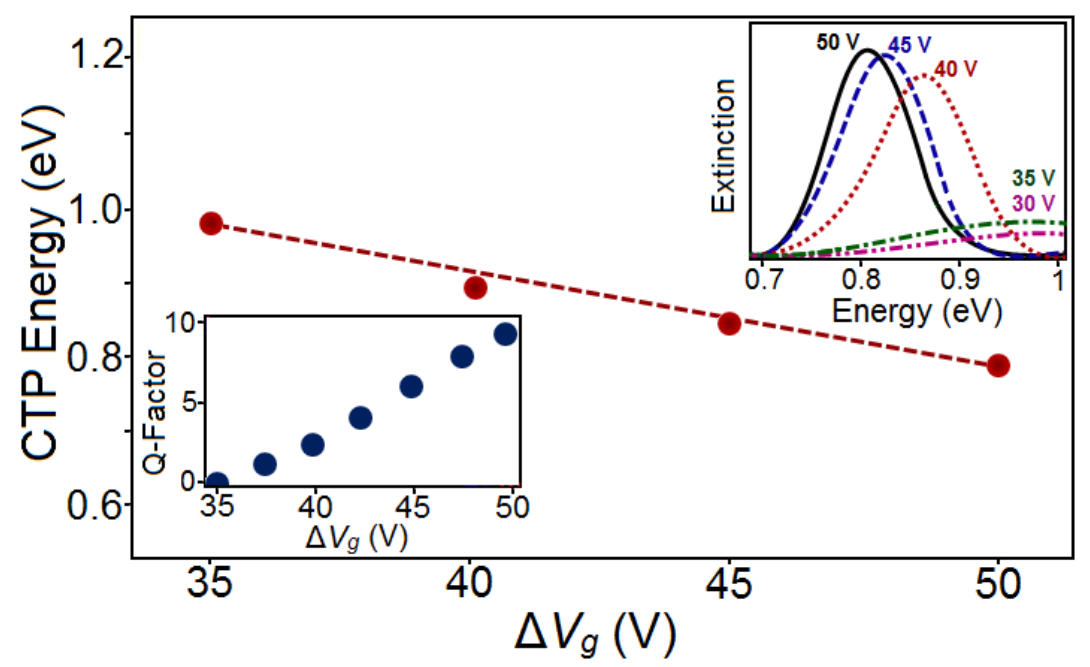

Figure 4.14. The CTP energy as a function of gate voltage variations $\left(\Delta V_{g}\right)$. The insets are the extinction diagram for the plasmonic nanoswitch for the gate voltage variations below the Dirac point and the quality factor of CTP peak for the gate voltage variations.

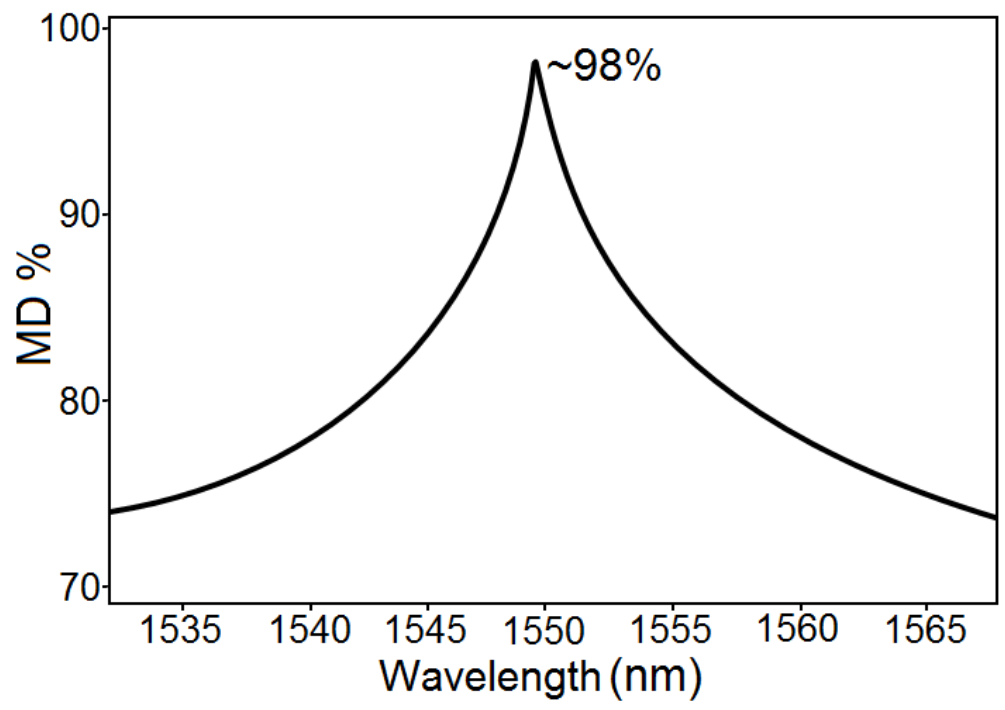

Figure 4.15. Numerically quantified MD of the graphene-plasmonic switch as a function of incident photon wavelength with higher resolution under back-gate bias application (at the Dirac point).

Possessing tunable CTP modes around the telecommunication band leads to using the graphene-plasmonic nanostructure as an optoelectronic nanodevice for NIR optical modulation. In this process, tunable conductivity of graphene determines the electromagnetic response of the structure and hence, the corresponding switching 
characteristics. As it was mentioned above, graphene with carrier density close to neutrality point acts similar to the semiconductors. Therefore, we expect formation of dipolar peak at high energies (here $\sim 2.7 \mathrm{eV}$ ) and elimination of CTP peak due to absence of conductive junction to transfer the charges (Off state). On the other hand, highly $n$ doped graphene with semi-metallic behavior shows high conductivity [54], and facilitates transfer of charges resulting excitation of CTP mode at the targeted wavelength (On state). Figure 4.13 demonstrates the normalized extinction spectra for the proposed device under incident beam illumination and varying back-gate bias. Obviously, in the absence of gate voltage, the CTP mode vanishes, while for the required applied bias and $n$-doped regime, a pronounced CTP peak appeared around $0.8 \mathrm{eV}(1.55 \mu \mathrm{m})$, shown by a dotted line in the plotted profile. The inset is the local E-field map for dipolar resonant mode $(\sim 2.7 \mathrm{eV})$ when the graphene sublayer is in semiconducting or intrinsic regime ( $V_{g}$ Off). The position and energy of CTP peak as a function of back-gate voltage variations (35 $\left.\mathrm{V} \leq \Delta V_{g} \leq 50 \mathrm{~V}\right)$ is shown in Fig. 4.14. As discussed earlier, the peak of CTP mode is located at $0.8 \mathrm{eV}$ for the applied voltage far away from the Dirac point with lower resistance and intense doping of graphene sheet $\left(V_{g}=50 \mathrm{~V}\right)$. Continuous reduction in the back-gate voltage leads to a blue-shift in the position of the resonant mode to higher energies due to reaching the intrinsic point and huge increase in the atomic sheet resistance. The insets in Figs. 4.14 provide more details for destructive results of gate-voltage variations. Here, the extinction spectra reveal how the CTP mode greatly damped for $\Delta V_{g} \leq 35$ $\mathrm{V}$ (reaching the intrinsic level). This originates due to significant reduction in the carrier concentration (low doping regime) and the conductance of the atomic 
carbon bridge. The other inset profile demonstrates the quality factor of CTP mode as a function of gate voltage variations, confirming the drastic decay of CTP mode by reducing the bias voltage and losing the conductivity of junction. Here, the quality factor is quantified as the ratio of resonance wavelength to the full width at half maximum (FWHM). Figure 4.15 shows the MD of the switch as a function of incident light wavelength. This profile confirms the archived high modulation depth (MD) up to $98 \%$ at the global telecommunication wavelength (C-band) using functional CTP resonant mode. Such a high MD is comparable with and higher that analogous plasmons inspired graphene-based, molecular, and atomic alloptical and optoelectronic switches [54]. The framework developed in this study would allow realizing ultrafast, high-quality devices for advanced and next-generation integrated nanophotonic technology.

\subsection{References}

1. Hentschel, M., Wu, L., Schäferling, M., Bai, P., Li, E. P., \& Giessen, H. (2012). Optical properties of chiral three-dimensional plasmonic oligomers at the onset of charge-transfer plasmons. ACS Nano, 6(11), 10355-10365.

2. Koya, A. N., \& Lin, J. (2017). Charge transfer plasmons: Recent theoretical and experimental developments. Applied Physics Reviews, 4(2), 021104.

3. Nooshnab, V., \& Ahmadivand, A. (2017). Optothermally functional charge transfer plasmon modulator. IEEE Photonics Technology Letters, 29(18), 1556-1559.

4. Ahmadivand, A., Gerislioglu, B., Sinha, R., Karabiyik, M., \& Pala, N. (2017). Optical switching using transition from dipolar to charge transfer plasmon modes in $\mathrm{Ge}_{2} \mathrm{Sb}_{2} \mathrm{Te}_{5}$ bridged metallodielectric dimers. Scientific Reports, 7, 42807.

5. Ahmadivand, A., Gerislioglu, B., \& Pala, N. (2017). Graphene optical switch based on charge transfer plasmons. Physica Status Solidi (RRL)-Rapid Research Letters.

6. Ahmadivand, A., Gerislioglu, B., Sinha, R., Vabbina, P. K., Karabiyik, M., \& Pala, N. (2017). Excitation of Terahertz Charge Transfer Plasmons in Metallic Fractal Structures. Journal of Infrared, Millimeter, and Terahertz Waves, 38(8), 992-1003. 
7. Savage, K. J., Hawkeye, M. M., Esteban, R., Borisov, A. G., Aizpurua, J., \& Baumberg, J. J. (2012). Revealing the quantum regime in tunnelling plasmonics. Nature, 491(7425), 574.

8. Tan, S. F., Wu, L., Yang, J. K., Bai, P., Bosman, M., \& Nijhuis, C. A. (2014). Quantum plasmon resonances controlled by molecular tunnel junctions. Science, 343(6178), 1496-1499.

9. Wu, L., Duan, H., Bai, P., Bosman, M., Yang, J. K., \& Li, E. (2012). FowlerNordheim tunneling induced charge transfer plasmons between nearly touching nanoparticles. ACS Nano, 7(1), 707-716.

10. Wen, F., Zhang, Y., Gottheim, S., King, N. S., Zhang, Y., Nordlander, P., \& Halas, N. J. (2015). Charge transfer plasmons: optical frequency conductances and tunable infrared resonances. ACS Nano, 9(6), 6428-6435.

11. Ahmadivand, A., Sinha, R., Gerislioglu, B., Karabiyik, M., Pala, N., \& Shur, M. (2016). Transition from capacitive coupling to direct charge transfer in asymmetric terahertz plasmonic assemblies. Optics Letters, 41(22), 5333-5336.

12. Wang, Y., Li, Z., Zhao, K., Sobhani, A., Zhu, X., Fang, Z., \& Halas, N. J. (2013). Substrate-mediated charge transfer plasmons in simple and complex nanoparticle clusters. Nanoscale, 5(20), 9897-9901.

13. Nooshnab, V., \& Golmohammadi, S. (2017). Revealing the effect of plasmon transmutation on charge transfer plasmons in substrate-mediated metallodielectric aluminum clusters. Optics Communications, 382, 354-360.

14. Novotny, L., \& Van Hulst, N. (2011). Antennas for light. Nature Photonics, 5(2), 8390.

15. Ciracì, C., Hill, R. T., Mock, J. J., Urzhumov, Y., Fernández-Domínguez, A. I., Maier, S. A., Pendry, J. B., Chilkoti, A. and Smith, D. R. (2012). Probing the ultimate limits of plasmonic enhancement. Science, 337(6098), 1072-1074.

16. Johansson, P. (1998). Light emission from a scanning tunneling microscope: Fully retarded calculation. Physical Review B, 58(16), 10823.

17. Bauernschmitt, R., \& Ahlrichs, R. (1996). Treatment of electronic excitations within the adiabatic approximation of time dependent density functional theory. Chemical Physics Letters, 256(4-5), 454-464.

18. Esteban, R., Zugarramurdi, A., Zhang, P., Nordlander, P., García-Vidal, F. J., Borisov, A. G., \& Aizpurua, J. (2015). A classical treatment of optical tunneling in 
plasmonic gaps: extending the quantum corrected model to practical situations. Faraday Discussions, 178, 151-183.

19. Esteban, R., Borisov, A. G., Nordlander, P., \& Aizpurua, J. (2012). Bridging quantum and classical plasmonics with a quantum-corrected model. Nature Communications, 3,825 .

20. Zugarramurdi, A., Zabala, N., Borisov, A. G., \& Chulkov, E. V. (2011). Theoretical study of constant current scanning tunneling spectroscopy in $\mathrm{Pb}$ overlayers. Physical Review B, 84(11), 115422.

21. Chulkov, E. V., Silkin, V. M., \& Echenique, P. M. (1999). Image potential states on metal surfaces: binding energies and wave functions. Surface Science, 437(3), 330352.

22. Blanco, J. M., Flores, F., \& Pérez, R. (2006). STM-theory: Image potential, chemistry and surface relaxation. Progress in Surface Science, 81(10), 403-443.

23. Pérez-González, O., Zabala, N., Borisov, A. G., Halas, N. J., Nordlander, P., \& Aizpurua, J. (2010). Optical spectroscopy of conductive junctions in plasmonic cavities. Nano Letters, 10(8), 3090-3095.

24. Schuller, J. A., Barnard, E. S., Cai, W., Jun, Y. C., White, J. S., \& Brongersma, M. L. (2010). Plasmonics for extreme light concentration and manipulation. Nature Materials, 9(3), 193-204.

25. Tassin, P., Zhang, L., Koschny, T., Economou, E. N., \& Soukoulis, C. M. (2009). Low-loss metamaterials based on classical electromagnetically induced transparency. Physical Review Letters, 102(5), 053901.

26. Jeans, J. H. (1908). The mathematical theory of electricity and magnetism. University Press, Cambridge.

27. Masina, B. N., Lafane, S., Wu, L., Akande, A. A., Mwakikunga, B., Abdelli-Messaci, S., Kerdja, T. \& Forbes, A. (2015). Phase-selective vanadium dioxide $\left(\mathrm{VO}_{2}\right)$ nanostructured thin films by pulsed laser deposition. Journal of Applied Physics, 118(16), 165308.

28. Vinod, E. M., Ramesh, K., \& Sangunni, K. S. (2015). Structural transition and enhanced phase transition properties of $\mathrm{Se}$ doped $\mathrm{Ge}_{2} \mathrm{Sb}_{2} \mathrm{Te}_{5}$ alloys. Scientific Reports, 5, 8050.

29. Matsunaga, T., Akola, J., Kohara, S., Honma, T., Kobayashi, K., Ikenaga, E., Jones, R. O., Yamada, N., Takata, M. \& Kojima, R. (2011). From local structure to nanosecond recrystallization dynamics in AgInSbTe phase-change materials. Nature Materials, 10(2), 129. 
30. Chen, Y. G., Kao, T. S., Ng, B., Li, X., Luo, X. G., Luk'yanchuk, B., Maier, S. A. \& Hong, M. H. (2013). Hybrid phase-change plasmonic crystals for active tuning of lattice resonances. Optics Express, 21(11), 13691-13698.

31. Cao, T., Wei, C., Simpson, R. E., Zhang, L., \& Cryan, M. J. (2014). Fast tuning of Fano resonance in metal/phase-change materials/metal metamaterials. Optical Materials Express, 4(9), 1775-1786.

32. Nienhuys, H. K., \& Sundström, V. (2005). Influence of plasmons on terahertz conductivity measurements. Applied Physics Letters, 87(1), 012101.

33. Lorentz, H. A. (1916). Theory of electrons. Teubner, Leipzig, Chap. 4.

34. Russo, U., Ielmini, D., \& Lacaita, A. L. (2007). Analytical modeling of chalcogenide crystallization for PCM data-retention extrapolation. IEEE Transactions on Electron Devices, 54(10), 2769-2777.

35. Baffou, G., \& Quidant, R. (2013). Thermo-plasmonics: using metallic nanostructures as nano-sources of heat. Laser \& Photonics Reviews, 7(2), 171-187.

36. Chen, X., Chen, Y., Yan, M., \& Qiu, M. (2012). Nanosecond photothermal effects in plasmonic nanostructures. ACS Nano, 6(3), 2550-2557.

37. Shportko, K., Kremers, S., Woda, M., Lencer, D., Robertson, J., \& Wuttig, M. (2008). Resonant bonding in crystalline phase-change materials. Nature Materials, 7(8), 653.

38. Burr, G. W., Breitwisch, M. J., Franceschini, M., Garetto, D., Gopalakrishnan, K., Jackson, B., Kurdi, B., Lam, C., Lastras, L. A., Padilla, A. \& Rajendran, B., 2010. Phase change memory technology. Journal of Vacuum Science \& Technology B, Nanotechnology and Microelectronics: Materials, Processing, Measurement, and Phenomena, 28(2), 223-262.

39. Li, P., Yang, X., Maß, T. W., Hanss, J., Lewin, M., Michel, A. K. U., Wuttig, M. \& Taubner, T. (2016). Reversible optical switching of highly confined phononpolaritons with an ultrathin phase-change material. Nature Materials, 15(8), 870-875.

40. Geim, A. K., \& Novoselov, K. S. (2007). The rise of graphene. Nature Materials, 6(3), 183-191.

41. Bao, Q., \& Loh, K. P. (2012). Graphene photonics, plasmonics, and broadband optoelectronic devices. ACS Nano, 6(5), 3677-3694. 
42. Frenzel, A. J., Lui, C. H., Shin, Y. C., Kong, J., \& Gedik, N. (2014). Semiconductingto-metallic photoconductivity crossover and temperature-dependent Drude weight in graphene. Physical Review Letters, 113(5), 056602.

43. Novoselov, K. S., Geim, A. K., Morozov, S. V., Jiang, D., Katsnelson, M. I., Grigorieva, I. V., Dubonos, S. V., \& Firsov, A. A. (2005). Two-dimensional gas of massless Dirac fermions in graphene. Nature, 438(7065), 197-200.

44. Grigorenko, A. N., Polini, M., \& Novoselov, K. S. (2012). Graphene plasmonics. Nature Photonics, 6(11), 749-758.

45. Horng, J., Chen, C. F., Geng, B., Girit, C., Zhang, Y., Hao, Z., Bechtel, H. A., Martin, M., Zettl, A., Crommie, M. F. \& Shen, Y. R. (2011). Drude conductivity of Dirac fermions in graphene. Physical Review B, 83(16), 165113.

46. Kim, J., Lim, S. C., Chae, S. J., Maeng, I., Choi, Y., Cha, S., Lee, Y. H. and Choi, H. (2013). Ultrafast zero balance of the oscillator-strength sum rule in graphene. Scientific Reports, 3, 2663.

47. Jablan, M., Buljan, H., \& Soljačić, M. (2009). Plasmonics in graphene at infrared frequencies. Physical Review B, 80(24), 245435.

48. Klimov, V. I. (2000). Optical nonlinearities and ultrafast carrier dynamics in semiconductor nanocrystals. J. Phys. Chem. B, 104(26), 6112-6123.

49. Fang, Z., Wang, Y., Schlather, A. E., Liu, Z., Ajayan, P. M., García de Abajo, F. J., Nordlander, P., Zhu, X. \& Halas, N. J. (2013). Active tunable absorption enhancement with graphene nanodisk arrays. Nano Letters, 14(1), 299-304.

50. Du, B., Lin, L., Liu, W., Zu, S., Yu, Y., Li, Z., Kang, Y., Peng, H., Zhu, X. \& Fang, Z. (2017). Plasmonic hot electron tunneling photodetection in vertical $\mathrm{Au}$-graphene hybrid nanostructures. Laser \& Photonics Reviews, 11(1), 1600148.

51. McCann, E., \& Koshino, M. (2013). The electronic properties of bilayer graphene. Reports on Progress in Physics, 76(5), 056503.

52. Low, T., \& Avouris, P. (2014). Graphene plasmonics for terahertz to mid-infrared applications. ACS Nano, 8(2), 1086-1101.

53. Koppens, F. H., Chang, D. E., \& García de Abajo, F. J. (2011). Graphene plasmonics: a platform for strong light-matter interactions. Nano Letters, 11(8), 3370-3377.

54. Song, S. M., \& Cho, B. J. (2013). Contact resistance in graphene channel transistors. Carbon Letters, 14(3), 162-170. 


\section{CHAPTER 5}

\section{Toroidal Moments}

The interaction of incident intense EM wave with matter in subwavelength regime has been established as a fundamental approach for developing advanced photonic devices including but not limited to telecommunication applications, data storage, biosensing, and information processing. As we mentioned in the earlier sections, the traditional result of such a process is the excitation of classical EM multipoles. Therefore, EM media can be represented by a set of point-like multipole sources $[1,2]$. It is well-acknowledged that traditional electric and magnetic multipoles can be successfully represented by oscillating charges and closed-loop currents, respectively [3]. This led to discovery of a third family of multipoles independent from conventional EM multipoles, introduced for the very first time by Zel'dovich in 1957, categorized in toroidal topology [4]. Accordingly, the toroidization concept was demonstrated and verified in nuclear and atomic physics [5], solid state physics [6], and classical electrodynamics [7]. For the latter case, in the classical electrodynamic limit, the recent experiments for the toroidal response of materials have shown that dynamic toroidal multipole patterns can be detected successfully in both biological and artificial objects [8-10].

In this chapter, we will first trace the progress of toroidal electrodynamics and excitation of dynamic toroidal multipoles in both theoretical and experimental viewpoints and numerical and experimental analysis results for our novel toroidal plasmonic metamaterials. By using the proposed approaches and methods from the literature and 
also by describing our developed toroidal unit cells, we numerically and experimentally explain the formation and spectral behavior of toroidal moments.

\subsection{Dynamic Toroidal Dipoles and Static Multipoles}

In this subsection, we summarize the excitation of static toroidal dipoles and multipoles using theoretical and numerical studies. Similar to the macroscopic electric polarization and magnetization proposed by Dubovik et al. [11], toroidal multipoles can be considered by an order parameter, known as toroidization or toroidal polarization. In this regime, one can consider the electric polarization as the electric dipole density and the magnetic polarization as the magnetic dipole density, where toroidization signifies the density of toroidal resonances [2]. In terms of condensed matters and nuclear physics, toroidization concept operates based on both time-reversal $(t \rightarrow-t)$ and space inversion $(r \rightarrow-r)$ symmetry $[11,12]$. In the macroscopic regime, media can display macroscopic toroidization, known as ferrotoroids, analogous to ferroelectrics and ferromagnets. In other words, although ferroelectrics break spatial inversion symmetry and ferromagnets break time-reversal symmetry, ferrotoroids with magnetic toroidization concurrently
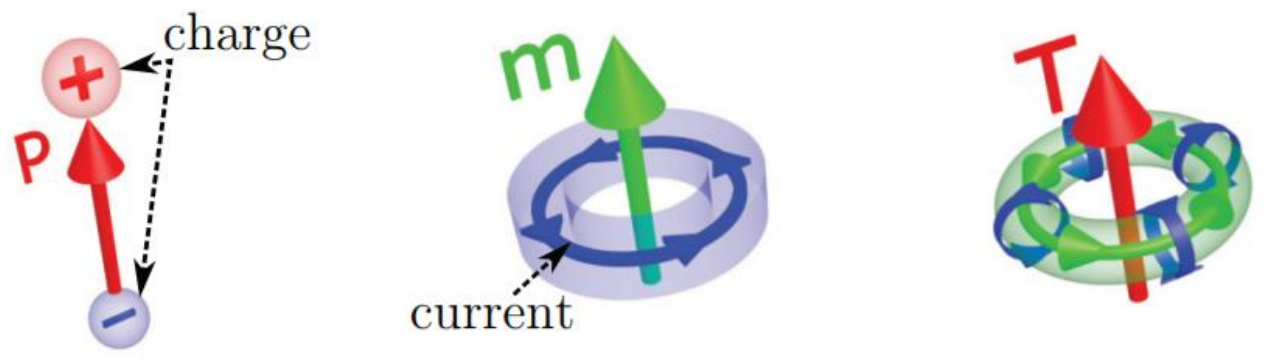

\section{Electric Multipoles Magnetic Multipoles Toroidal Multipoles}

Figure 5.1. Schematic for the EM dipoles (p and $\mathbf{m}$ ) and unconventional toroidal dipoles (T), obtained with permissions from APS, Ref. [14]. 
break both time-reversal and spatial inversion symmetry. Ferrotoroids exhibit electric polarization (magnetization) in response to an external magnetic (electric) field [13].

\subsubsection{Theory of Toroidal Dipolar Excitation}

The toroidal dipole is an unconventional EM excitation with concealed far-field signature masked by strong classical EM multipoles patterns that cannot be explained in terms of typical electric and magnetic multipoles principles [14]. Figure 5.1 illustrates an art picture of toroidal dipole or anapole as a first and fundamental member of toroidal multipoles and compares it with classical EM resonances [14]. As plotted in this picture, the toroidal dipole moment can be identified as a circular head-to-tail and closed-loop arrangement of magnetic dipoles, all squeezed into a single spot [11]. The unique complexity of the current distribution leads to the excitation of toroidal dipole moments in the highly confined high-quality resonant modes of the integrated metallic and alldielectric scatterers or resonators. So far, toroidal dipoles and other complex multipoles have successfully been experienced and observed in molecular structures $[15,16]$, ferroelectric systems $[13,17]$, microwave and $\mathrm{THz}$ metamaterials $[18,19]$, and optical frequency metasurfaces [20,21].

In continue, we analyze the theory of the excitation of toroidal dipole moments arising from an infinite 2D or planar periodic array of arbitrary subwavelength resonators or unit cells. To this end, using the homogenized Maxwell's equations, one needs to study the EM properties of the media in terms of macroscopic material parameters such as dielectric permittivity and magnetic permeability, etc. [14]. This allows for establishing a connection between the macroscopic EM response arisen from media and 
the microscopic charge- current excitations induced in atomic or molecular level media $[14,22]$. By assuming an array of planar multipixel resonators and using the proposed theoretical mechanism by Radescu et al. [23], the toroidal dipole can be expressed as a function of electric and magnetic moments:

$$
\overrightarrow{\mathbf{T}}=\overrightarrow{\mathbf{p}} \times \overrightarrow{\mathbf{m}}=\sum_{j} q_{j} \vec{r}_{j} \times\left(\vec{r}_{j} \times d \vec{r}_{j} / d t\right)
$$

Relatively, Gostescu et al. [24] have shown that the most general distribution of charge and currents stated by the charge $(\rho)$ and current $(j)$ densities can be expressed as a continuity relation:

$$
\frac{\partial \rho(\vec{r}, t)}{\partial t}+\vec{\nabla} \cdot \vec{j}(\vec{r}, t)=0
$$

Parametrizing the above equation is terms of three components including electric $\left(Q_{l m}(-\right.$ $\left.\left.k^{2}, t\right)\right)$, magnetic $\left(M_{l m}\left(-k^{2}, t\right)\right)$, and toroidal $\left(T_{l m}\left(-k^{2}, t\right)\right)$ multipoles allows to express the charge and current densities as [24]:

$$
\begin{gathered}
\rho(\vec{r}, t)=\frac{2}{8 \pi^{3}} \sum_{l, m, k}(-i k)^{l} \frac{\sqrt{4 \pi(2 l+1)}}{(2 l+1) ! !} \times Q_{l m}\left(-k^{2}, t\right) F_{l m k}(\vec{r}) \\
\vec{j}(\vec{r}, t)=\frac{2}{8 \pi^{3}} \sum_{l, m, k}(-i k)^{l-1} \frac{\sqrt{4 \pi(2 l+1)(l+1)}}{\sqrt{l}(2 l+1) ! !} \\
\times\left\{k M_{l m}\left(-k^{2}, t\right) \vec{F}_{l m k}^{(0)}(\vec{r})+\left[\frac{1}{c} \dot{Q}_{l m}(0, t)+k^{2} T_{l m}\left(-k^{2}, t\right)\right] \times \vec{F}_{l m k}^{(+)}+\frac{1}{c} \sqrt{\frac{l}{l+1}} \dot{Q}_{l m}\left(-k^{2}, t\right) \vec{F}_{l m k}^{(-)}(\vec{r})\right\}
\end{gathered}
$$

where 


$$
\sum_{k}=\int_{0}^{\infty} k^{2} d k \quad ;\{m=-l, \ldots, l, \quad l=0,1,2, \ldots\}
$$

In the equations above and in the upcoming relations, the sum over $l$ starts at $l=0$ for the electric multipole and $l=1$ for magnetic and toroid multipoles. The dot over $Q_{l m}$ defines derivation with respect to $t$. Finally, the factor of $F_{l m k}(\vec{r})$ is the system of regular solutions of the Helmholtz equation:

$$
\begin{gathered}
\left(\Delta+k^{2}\right) F_{l m k}(\vec{r})=0 \\
F_{l m k}(\vec{r})=j_{l}(k r) Y_{l m}(\vec{n}), \quad \vec{n}=\frac{\vec{r}}{r} \\
j_{l}(k r)=\sqrt[3]{2 \pi} i^{l} \frac{J_{l+1 / 2}(k r)}{\sqrt{k r}}
\end{gathered}
$$

where $j_{l}$ and $j_{l+l / 2}$ are the spherical and cylindrical Bessel functions, respectively and $Y_{l m}$ are the classical spherical harmonics. Here, the normalized of Helmholtz factor can be written as:

$$
\begin{gathered}
\int F_{l m k}(\vec{r}) F^{*} *_{l^{\prime} m^{\prime} k^{\prime}}(\vec{r}) d^{3} r=\delta_{l l^{\prime}} \delta_{m m^{\prime}} \frac{8 \pi^{3}}{k^{2}} \delta\left(k-k^{\prime}\right) \\
\sum_{l, m, k} F_{l m k}(\vec{r}) F_{l^{\prime} m^{\prime} k^{\prime}}(\vec{r})=8 \pi^{3} \delta\left(\vec{r}-\vec{r}^{\prime}\right) \\
\sum_{k}=\int_{0}^{\infty} k^{2} d k \\
F_{l m k}(-\vec{r})=(-1)^{l} F_{l m k}(\vec{r})
\end{gathered}
$$


And the basis factor function can be found as:

$$
\begin{gathered}
\left(\Delta+k^{2}\right) \vec{F}_{l m k}(\vec{r})=0 \\
\vec{F}_{l m k}^{(0)}(\vec{r})=\frac{i}{\sqrt{l(l+1)}} \vec{\nabla} \times\left[\vec{r} F_{l m k}(\vec{r})\right]=j_{l}(k r) \vec{Y}_{l l m}(\vec{n}) \\
\vec{F}_{l m k}^{(+)}(\vec{r})=\frac{-1}{\sqrt{l(l+1)}} \frac{i}{k} \vec{\nabla} \times \vec{\nabla} \times\left[\vec{r} F_{l m k}(\vec{r})\right] \\
=\frac{1}{\sqrt{2 l+1}}\left\{\sqrt{l} j_{l+1}(k r) \vec{Y}_{l l+1 m}(\vec{n})+\sqrt{l+1} j_{l-1}(k r) \vec{Y}_{l l-1 m}(\vec{n})\right\} \\
\vec{F}_{l m k}^{(-)}(\vec{r})=-\frac{i}{k} \vec{\nabla} F_{l m k}(\vec{r}) \\
=\frac{1}{\sqrt{2 l+1}}\left\{\sqrt{l} j_{l-1}(k r) \vec{Y}_{l l-1 m}(\vec{n})-\sqrt{l+1} j_{l+1}(k r) \vec{Y}_{l l+1 m}(\vec{n})\right\}
\end{gathered}
$$

The corresponding spherical vectors are described in details in Ref. [24]. Accordingly, the EM and toroidal multipolar form factors can be defined as a function of the functions described previously as:

$$
\begin{aligned}
& Q_{l m}\left(-k^{2}, t\right)=\frac{(2 l+1) ! !}{(-i k)^{l} \sqrt{4 \pi(2 l+1)}} \int \rho(\vec{r}, t) j_{l}^{*}(k r) Y_{l}^{*}(\vec{n}) d^{3} r \\
& M_{l m}\left(-k^{2}, t\right)=\frac{-i(2 l+1) ! !}{c(-i k)^{l} \sqrt{4 \pi(2 l+1) \frac{(l+1)}{l}}} \times \int \vec{j}(\vec{r}, t) j_{l}^{*}(k r) Y_{l l m}^{*}(\vec{n}) d^{3} r
\end{aligned}
$$




$$
\begin{aligned}
& T_{l m}\left(-k^{2}, t\right)=\frac{-(2 l-1) ! ! \sqrt{l}}{c(-i k)^{l+1} \sqrt{4 \pi(l+1)}} \\
& \times \int\left\{\sqrt{l} j_{l+1}^{*}(k r) Y_{l l+1 m}^{*}(\vec{n}) \sqrt{l+1}+\left[j_{l-1}^{*}(k r)-\frac{4 \pi(-i k r)^{l-1}}{(2 l-1) ! !}\right] \times Y_{l l-1 m}^{*}(\vec{n})\right\} \vec{j}(\vec{r}, t) d^{3} r
\end{aligned}
$$

where

$$
F_{l m k}(\vec{r}) \sim \lim _{r \rightarrow 0} \frac{4 \pi(i k r)^{l}}{(2 l+1) ! !} Y_{l m}(\vec{n})
$$

The conjugate of the equations above can be obtained using the following relations:

$$
\left\{\begin{array}{l}
Q_{l m}^{*}\left(-k^{2}, t\right)=(-1)^{m} Q_{l,-m}\left(-k^{2}, t\right) \\
M_{l m}^{*}\left(-k^{2}, t\right)=(-1)^{m} M_{l,-m}\left(-k^{2}, t\right) \\
T_{l m}^{*}\left(-k^{2}, t\right)=(-1)^{m} T_{l,-m}\left(-k^{2}, t\right)
\end{array}\right.
$$

Next, to calculate the radiation intensity arising from resonators at large distances, one needs to find the exact descriptions for the electric and magnetic fields by considering their behavior at large distances. To this end, we use the extracted electric and magnetic fields emitted from a unit cell in terms of Fourier transforms in time:

$$
\begin{aligned}
& \vec{E}(\vec{r}, t)=\frac{1}{\sqrt[3]{\pi}} \\
& \int_{0}^{\infty}\left\{\sum_{l, m}(-i)^{l-1}\left(\frac{\omega}{c}\right)^{l+2} \frac{\sqrt{2 l+1}}{(2 l+1) ! !}\left[\sin (\omega t) \vec{N}_{l m(\omega / c)}^{(-)}(\vec{r})+\cos (\omega t) \vec{F}_{l m(\omega / c)}^{(-)}(\vec{r})\right] Q_{l m}\left(-\frac{\omega^{2}}{c^{2}}, \omega\right)\right\} d \omega \\
& -\frac{1}{2 \pi^{2} c} \int_{0}^{\infty} \sum_{l m}(-i)^{l-1}\left(\frac{\omega}{c}\right)^{l+1} \frac{\sqrt{4 \pi(2 l+1)(l+1)}}{\sqrt{l}(2 l+1) ! !}\left\{\left[\sin (\omega t) \vec{N}_{l m(\omega / c)}^{(-)}(\vec{r})+\cos (\omega t) \vec{F}_{l m(\omega / c)}^{(-)}(\vec{r})\right]\right. \\
& \times \frac{\sqrt{l}}{\sqrt{l+1}} \omega Q_{l m}\left(-\frac{\omega^{2}}{c^{2}}, \omega\right)+\omega\left[\sin (\omega t) \vec{N}_{l m(\omega / c)}^{(0)}(\vec{r})+\cos (\omega t) \vec{F}_{l m(\omega / c)}^{(0)}(\vec{r})\right] M_{l m}\left(-\frac{\omega^{2}}{c^{2}}, \omega\right) \\
& \left.+\left[\sin (\omega t) \vec{N}_{l m(\omega / c)}^{(+)}(\vec{r})+\cos (\omega t) \vec{F}_{l m(\omega / c)}^{(+)}(\vec{r})\right]\left[\omega Q_{l m}(0, \omega)+\frac{\omega^{2}}{c} T_{l m}\left(-\frac{\omega^{2}}{c^{2}}, \omega\right)\right]\right\} d \omega
\end{aligned}
$$




$$
\begin{aligned}
& \vec{B}(\vec{r}, t)=\frac{1}{2 \pi^{2}} \\
& \int_{0}^{\infty} \sum_{l, m}(-i)^{l-1}\left(\frac{\omega}{c}\right)^{l+2} \times \frac{\sqrt{4 \pi(2 l+1)(l+1)}}{\sqrt{l}(2 l+1) ! !}\left\{\left[-\sin (\omega t) \vec{F}_{l m(\omega / c)}^{(+)}(\vec{r})+\cos (\omega t) \vec{N}_{l m(\omega / c)}^{(+)}(\vec{r})\right.\right. \\
& M_{l m}\left(-\frac{\omega^{2}}{c^{2}}, \omega\right)+\left[-\sin (\omega t) \vec{F}_{l m(\omega / c)}^{(0)}(\vec{r})+\cos (\omega t) \vec{N}_{l m(\omega / c)}^{(0)}(\vec{r})\right] \\
& \left.\times\left[Q_{l m}(0, \omega)+\frac{\omega^{2}}{c} T_{l m}\left(-\frac{\omega^{2}}{c^{2}}, \omega\right)\right]\right\} d \omega
\end{aligned}
$$

By computing the EM fields in the order of $r \rightarrow \infty$, we have:

$$
\begin{aligned}
& \vec{E}(\vec{r}, t) \sim \frac{1}{r} \sum_{l m}\left(\frac{1}{c}\right)^{l+1} \frac{\sqrt{4 \pi(2 l+1)(l+1)}}{\sqrt{l}(2 l+1) ! !} \times\left\{-Q_{l m}^{(0)(l+1)}\left(0, t-\frac{r}{c}\right)\right. \\
& \times\left[\frac{\sqrt{l}}{\sqrt{2 l+1}} \vec{Y}_{l l+1 m}(\vec{n})+\frac{\sqrt{l+1}}{\sqrt{2 l+1}} \vec{Y}_{l l-1 m}(\vec{n})\right]-\sum_{n=0}^{\infty} \frac{1}{n ! c^{2 n}} M_{l m}^{(n)(l+2 n+1)}\left(0, t-\frac{r}{c}\right) \vec{Y}_{l l m}(\vec{n}) \\
& +\frac{1}{c} \sum_{n=0}^{\infty} \frac{1}{n ! c^{2 n}} T_{l m}^{(n)(l+2 n+2)}\left(0, t-\frac{r}{c}\right) \times\left[\frac{\sqrt{l}}{\sqrt{2 l+1}} \vec{Y}_{l l+1 m}(\vec{n})+\frac{\sqrt{l+1}}{\sqrt{2 l+1}} \vec{Y}_{l l-1 m}(\vec{n})\right] \\
& \vec{B}(\vec{r}, t) \sim \frac{1}{r} \sum_{l m}\left(\frac{1}{c}\right) \frac{\sqrt{4 \pi(2 l+1)(l+1)}}{\sqrt{l}(2 l+1) ! !} \\
& \times\left\{-i Q_{l m}^{(0)(l+1)}\left(0, t-\frac{r}{c}\right) \vec{Y}_{l l m}(\vec{n}) \times \sum_{n=0}^{\infty} \frac{1}{n ! c^{2 n}} M_{l m}^{(n)(l+2 n+1)}\left(0, t-\frac{r}{c}\right) \vec{Y}_{l l m}(\vec{n})\right. \\
& \left.\times\left[\frac{\sqrt{l}}{\sqrt{2 l+1}} \vec{Y}_{l l+1 m}(\vec{n})+\frac{\sqrt{l+1}}{\sqrt{2 l+1}} \vec{Y}_{l l-1 m}(\vec{n})\right]+\frac{1}{c} \sum_{n=0}^{\infty} \frac{1}{n ! c^{2 n}} T_{l m}^{(n)(l+2 n+2)}\left(0, t-\frac{r}{c}\right)\right\}
\end{aligned}
$$

Employing the Eq. 5.24, we firstly extract the far-field radiation pattern of the electric field scattered from a single oscillating toroidal dipole as follows [14,23-25]:

$$
E(\mathbf{r})=\frac{-i \mu_{0} c^{2} k^{3}}{3 \sqrt{2 \pi} r} \exp (-i k r) \times \sum_{m=0, \pm 1} T_{1 m}\left[\mathbf{Y}_{1,2, m}+\sqrt{2} \mathbf{Y}_{1,0, m}\right]
$$




$$
\begin{aligned}
& T_{1, \pm 1}=\frac{1}{\sqrt{2}}\left(\mp T_{x}+i T_{y}\right) \\
& T_{1,0}=T_{z} \\
& \mathbf{T}=\frac{1}{10 c} \int\left[\mathbf{r}(\mathbf{r} . \mathbf{J})-2 r^{2} \mathbf{J}\right] d^{3} r
\end{aligned}
$$

where $\mu_{0}$ is the magnetic permeability of vacuum, $\mathbf{r}$ is the connecting vector between the location of the dipole moment with the detector. Here, $\mathbf{T}$ resembles the toroidal dipole mode and $\mathbf{J}$ is the current density. The total field radiated by a metasurface consists of infinite arrays of resonators and toroidal dipoles $\left(\mathbf{E}_{\mathbf{s}}\right)$ can be obtained by the summation of contributions from all dipoles at the position of the observer. We assumed all excited dipoles are oscillating in-phase and the metamolecule arrays are adequately smaller than the incident beam wavelength. Then, we presumed that the complex-valued field emitted by the single emitter at $\mathbf{r}_{s}$ and detected by the detector at point $\mathbf{r}_{d}$ is given by [14]:

$$
A_{k, l, m}^{(s)}\left(\mathbf{r}_{d} ; \mathbf{r}_{s}\right)=Y_{l, m}\left(\frac{\mathbf{r}_{d}-\mathbf{r}_{s}}{\left|\mathbf{r}_{d}-\mathbf{r}_{s}\right|}\right) \frac{\exp \left(-i k\left|\mathbf{r}_{d}-\mathbf{r}_{s}\right|\right)}{\left|\mathbf{r}_{d}-\mathbf{r}_{s}\right|}
$$

in which $k$ is the wave number and as we discussed previously, $\mathbf{Y}$ denotes the spherical harmonics [26]. In this limit, the total field reaching the detector at distance $R$ away from the array will be:

$$
A_{k, l, m}^{(d)}\left(x_{d}, y_{d}, R\right)=\sum_{n} A_{k, l, m}^{(s)}\left(x_{d}, y_{d}, R ; \mathbf{r}_{n}\right)
$$


Now, using this assumption that the array lies in the $x y$-plane at $z=0$ and the detector is located at $z=R$, we can substitute the sum over the metamolecules of the array with following integral:

$$
\mathbf{E}_{\mathrm{s}}=\sum_{\mathbf{r}} \mathbf{E}(\mathbf{r}) \approx \frac{1}{\Delta^{2}} \int \mathbf{E}(\mathbf{r}) d^{2} r
$$

where $\Delta$ is the area of the metamolecule. Then, by carrying out the integration, we have:

$$
A_{k, l, m}^{(d)}\left(x_{d}, y_{d}, R\right) \cong \frac{\pi \delta_{m, 0}}{i k \Delta^{2}} \sqrt{\frac{2 l+1}{\pi}} \exp (-i k R)
$$

In continue, by assuming that the propagation direction of radiation occurs in a space with negligible losses and by focusing on the far-field component of the radiation for the distances much bigger than the incident wave wavelength, one can claim that:

$$
I_{l, m} \cong \frac{\pi \delta_{m, 0}(\hat{\mathbf{R}} \cdot \hat{\mathbf{z}})^{l}}{i k} \sqrt{\frac{2 l+1}{\pi}} \exp (-i k R)
$$

By substituting the Eq. 5.26 into Eq. 5.32 and utilizing Eq. 5.34, we have:

$$
\mathbf{E}_{\mathrm{s}}=\frac{\mu_{0} c^{2} k^{2}}{4 \Delta^{2}} \sqrt{2}\left(\begin{array}{l}
T_{1,1}-T_{1,-1} \\
i\left(T_{1,1}-T_{1,-1}\right) \\
0
\end{array}\right) \exp (-i k R)
$$

More simplification of the equation above leads to the final form of the scattered field as [14]:

$$
\mathbf{E}_{\mathbf{s}}=-\frac{\mu_{0} c^{2} k^{2}}{2 \Delta^{2}} \mathbf{T}_{\|} \exp (-i k R)
$$


The projected toroidal dipole $\left(\mathbf{T}_{\|}\right.$) from the $x y$-plane is given by: $\mathbf{T}_{\|}=T_{x} \hat{\mathbf{x}}+T_{y} \hat{\mathbf{y}}$ or in general: $\quad \mathbf{T}_{\|}=\mathbf{T}-(\mathbf{T} \cdot \hat{\mathbf{R}}) \hat{\mathbf{R}}$. To derive the far-field distributions for other isolated multipoles, one needs to use the expression for the radiation emitted by single multipole sources [23]. Due to the large number of terms, it would be better to separate the series into different orders of $l$. We listed three of the dipolar $(l=1)$, quadrupolar $(l=2)$, and $\operatorname{octupolar}(l=3)$ contributions here:

$$
\begin{aligned}
\mathbf{E}_{(l=1)} \approx & \frac{\mu_{0} c^{2}}{3 \sqrt{2 \pi}} \frac{\exp (-i k R)}{r} \times \sum_{m=0, \pm 1}\left\{\left(k^{2} Q_{1, m}-i k^{3} T_{1, m}+i k^{5} T_{1, m}^{(1)}\right) \times\left(\mathbf{Y}_{1,2, m}+\sqrt{2} \mathbf{Y}_{1,0, m}\right)\right. \\
& \left.+\mathrm{i} \sqrt{3}\left(k^{2} M_{1, m}-k^{4} T_{1, m}^{(1)}\right) \times \mathbf{Y}_{1,1, m}\right\} \\
\mathbf{E}_{(l=2)} \approx & \frac{\mu_{0} c^{2}}{10 \sqrt{6 \pi}} \frac{\exp (-i k R)}{r} \times \sum_{m=0, \pm 1, \pm 2}\left\{\left(i k^{2} Q_{2, m}^{(e)}+k^{5} Q_{2, m}^{(T)}\right) \times\left(\sqrt{2} \mathbf{Y}_{2,3, m}+\sqrt{3} \mathbf{Y}_{2,1, m}\right)\right. \\
& \left.-\mathrm{i} \sqrt{5} k^{3} Q_{2, m}^{(m)} \mathbf{Y}_{2,2, m}\right\} \\
\mathbf{E}_{(l=3)} \approx & -\frac{\mu_{0} c^{2} k^{4}}{15 \sqrt{3 \pi}} \frac{\exp (-i k R)}{r} \times \sum_{m=0, \pm 1, \pm 2, \pm 3}\left\{\frac{1}{7} O_{3, m}^{(e)}\left(\sqrt{3} \mathbf{Y}_{3,4, m}+2 \mathbf{Y}_{3,2, m}\right)+\frac{\mathrm{i}}{\sqrt{7}} O_{3, m}^{(m)} \mathbf{Y}_{3,3, m}\right\}
\end{aligned}
$$

The total emitted field is given by:

$$
\mathbf{E}=\mathbf{E}_{(l=1)}+\mathbf{E}_{(l=2)}+\mathbf{E}_{(l=3)}+\ldots
$$

It should be underline that the terms above $l>3$ (i.e. hexadecapole, etc.) can be neglected due to weak impact on the total emitted electric field. Ultimately, the far-field for the electric field propagation can be written as [14]: 


$$
\begin{aligned}
\mathbf{E}_{s} & =\frac{\mu_{0} c^{2}}{2 \Delta^{2}}\left\{-i k \mathbf{p}_{\|}+i k \hat{\mathbf{R}} \times\left[\mathbf{m}_{\|}-\frac{k^{2}}{10} \mathbf{m}_{\|}^{(1)}\right]-k^{2}\left[\mathbf{T}_{\|}-\frac{k^{2}}{10} \mathbf{T}_{\|}^{(1)}\right]+k^{2}\left[\mathbf{Q}^{(\mathbf{e})} \cdot \hat{\mathbf{R}}\right]_{\|}\right. \\
& \left.-\frac{k^{2}}{2} \hat{\mathbf{R}} \times\left[\mathbf{Q}^{(\mathbf{m})} \cdot \hat{\mathbf{R}}\right]_{\|}-\frac{i k^{3}}{3}\left[\mathbf{Q}^{(\mathbf{T})} \cdot \hat{\mathbf{R}}\right]_{\|}-i k^{3}\left[\left(\mathbf{O}^{(\mathbf{e})} \cdot \hat{\mathbf{R}}\right) \hat{\mathbf{R}}\right]_{\|}-\frac{i k^{3}}{180}\left[\left(\mathbf{O}^{(\mathbf{m})} \cdot \hat{\mathbf{R}}\right) \hat{\mathbf{R}}\right]_{\|}\right\} \times \exp (-i k R)
\end{aligned}
$$

The equation above leads to qualitative calculation of electric field emitted by a surface composed of planar resonators. The contributed terms to the emitted field are electric (p), magnetic $(\mathbf{m})$, and toroidal dipoles $(\mathbf{T})$, electric $\left(\mathbf{Q}^{(\mathbf{e})}\right)$, magnetic $\left(\mathbf{Q}^{(\mathbf{m})}\right)$, and toroidal $\left(\mathbf{Q}^{(\mathbf{T})}\right)$ quadrupoles, electric $\left(\mathbf{O}^{(\mathbf{e})}\right)$, magnetic $\left(\mathbf{O}^{(\mathbf{m})}\right)$, and toroidal $\left(\mathbf{O}^{(\mathbf{T})}\right)$ octupoles. The mean-square radii of toroidal and magnetic dipoles are denoted by $\mathbf{T}^{(1)}$ and $\mathbf{m}^{(1)}$, respectively.

\subsubsection{The Toroidal Multipoles Excitation Power}

As it was discussed theoretically in the previous sections, toroidal resonances were induced with high-quality in artificially fabricated all-dielectric and plasmonic metamaterials composed of both planar and 3D metamolecules along the visible to the microwave frequencies. The quality, frequency, and order of toroidal resonant moments strongly depend on the geometry of the metamolecules. In order to confirm the multipole nature of these resonant modes, an analytical model of multipole expansion can be utilized [23]. In principle, either the transmission or reflection spectra can be disintegrated into radiating contribution of multipoles, including all the electric, magnetic, and toroidal modes. The localized distribution of the volume current density $(\boldsymbol{J})$ in a random metamolecule can be utilized to analyze the resonant moments properties in terms of multipoles scattering contributions as follows [23,27]: 


$$
\begin{aligned}
& \mathrm{P}=\frac{1}{i \omega} \int j d^{3} r \\
& \mathrm{M}=\frac{1}{2 c} \int(\mathrm{r} \times \mathrm{j}) d^{3} r \\
& \mathrm{~T}=\frac{1}{10 c} \int\left[(\mathrm{r} . \mathrm{j}) \mathrm{r}-2 \mathrm{r}^{2} \mathrm{j}\right] d^{3} r \\
& \mathrm{Q}_{\alpha \beta}=\frac{1}{i 2 \omega} \int\left(r_{\alpha} j_{\beta}+r_{\beta} j_{\alpha}-\frac{2}{3}[\mathrm{r} . \mathrm{j}] \delta_{\alpha \beta}\right) d^{3} r \\
& \mathrm{M}_{\alpha \beta}=\frac{1}{3 c} \int\left([i \times j]_{\alpha} r_{\beta}+[i \times j]_{\beta} r_{\alpha}\right) d^{3} r
\end{aligned}
$$

where $\mathrm{P}, \mathrm{M}, \mathrm{T}, Q_{\alpha \beta}$, and $\mathrm{M}_{\alpha \beta}$ are the electric dipole, magnetic dipole, toroidal dipole, electric quadrupole, and magnetic quadrupole moments, respectively, and also, $\alpha \beta=x, y, z$. the scattering field power for each moment is given by [21]:

$$
\begin{aligned}
& I_{P}=\frac{2 \omega^{4}|\mathbf{P}|^{2}}{3 c^{3}} \\
& I_{M}=\frac{2 \omega^{4}|\mathbf{M}|^{2}}{3 c^{3}} \\
& I_{T}=\frac{2 \omega^{6}|\mathbf{T}|^{2}}{3 c^{5}} \\
& I_{Q}^{e}=\frac{\omega^{6}}{5 c^{5}} \sum\left|\mathbf{Q}_{\alpha \beta}\right|^{2}
\end{aligned}
$$




$$
I_{Q}^{m}=\frac{\omega^{6}}{40 c^{5}} \sum\left|\mathbf{M}_{\alpha \beta}\right|^{2}
$$

In addition, the scattering intensity of the octupolar moment is given by: $I_{O}=1 / c^{5} \mathbf{O}$. It should be noted that the engineering of artificial metamolecules, the dielectric function, and the environmental components play fundamental role in determining the intensity of the toroidal moments and also its performance

In the next section, we present numerical and experimental studies for the excitation of toroidal multipoles in plasmonic metasurfaces designed, fabricated and analyzed as part of this dissertation work.

\subsubsection{Numerical and Experimental Observation of Toroidal Moments}

In this subsection, we provide our numerical and experimental data for the observation of toroidal moments in plasmonic metamaterials consist of multipixel planar metamolecules. Although it is verified that pronounced toroidal moments can be excited in 3D unit cells, the fabrication of these structures, especially in the nanoscale dimensions is challenging and costly $[28,29]$. Therefore, the excitation of strong toroidal moments in planar unit cells with easier electron beam lithography (EBL) and classical photolithography techniques allows for developing efficient and optimized plasmonic devices such as sensors and modulators. Here, the radiation transmitted from a planar array of metamolecules can be found by employing the following relation [30]:

$$
\mathbf{E}_{\mathrm{T}}=\left.\mathbf{E}_{\mathrm{s}}\right|_{\hat{R}=\hat{k}}+\mathbf{E}_{\mathrm{I}}
$$



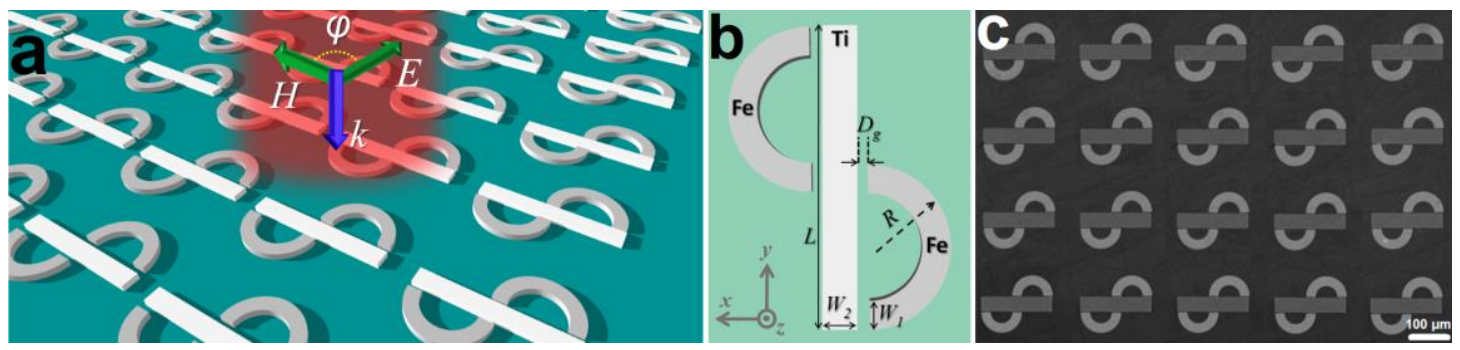

Figure 5.2. a) Graphical representation of bimetallic plasmonic metamolecules on a silicon host under $\mathrm{THz}$ beam illumination. b) An introduction to the geometrical components of unit cell. c) The SEM image of fabricated plasmonic multipixel structures in arrays with the gap spots between surrounding and central resonators of $D_{g}=3 \mu \mathrm{m}$, while the other geometries are fixed to: $L=240 \mu \mathrm{m}, R=50 \mu \mathrm{m}, W_{l}=30 \mu \mathrm{m}$, and $W_{2}=40 \mu \mathrm{m}$.

where $\mathbf{E}_{\mathrm{I}}$ is the incident field intensity, and $k$ is the unit vector points in the propagation direction, which is presumed in the $z$-axis in the following demonstrations. Figures 5.2a and $5.2 \mathrm{~b}$ represent the schematic and top-view images of the proposed multipixel plasmonic unit cell with the description of geometrical parameters. As plotted in the associated profile, we use a bimetallic and planar arrangement of $\mathrm{Fe}$ and $\mathrm{Ti}$ resonators, respectively, to design a multipixel metamolecule composed of asymmetric resonators to support ultrastrong and sharp toroidal dipolar momentum across the sub- $\mathrm{THz}$ spectrum. With coupled-resonator effects, the magnetic nature of $\mathrm{Fe}$ resonators intensifies resonating circular currents around the central block of the plasmonic metamolecule. Therefore, the middle Ti rectangle acts as a meridian for oscillation of closed-loop, headto-tail array of magnetic dipoles. Exploiting the polarization-dependency of the induced toroidal dipole to the incident magnetic component, we analyze and estimate the switching properties of the proposed plasmonic multipixel metamolecule numerically and experimentally. Figure 5.2c illustrates an SEM image of the fabricated bimetallic metamolecules on a high-resistivity silicon wafer with the gap distance of $D_{g}=3 \mu \mathrm{m}$ between peripheral and central resonators. It should be underlined that three different 
samples with different gap distances $(3 / 4 / 5 \mu \mathrm{m})$ were fabricated and analyzed here. For the fabrication of the proposed multipixel devices, we developed a two-level lithography microfabrication process. An undoped and high-resistivity $(>10 \mathrm{k} \Omega \mathrm{cm})$ silicon wafer with the crystal orientation of $\langle 100\rangle$ and thickness of $500 \mu \mathrm{m}$ was used as a substrate. We deposited $2 \mu \mathrm{m}$ negative photoresists (NLOF 2020) and patterned in two different steps. Employing e-beam evaporation, we then deposited $300 \mathrm{~nm}$ of Fe and Ti layers separately with the rate of $2 \AA / s$ (99.99\% purity for $\mathrm{Ti}$ and $99.95 \%$ purity for $\mathrm{Fe}$, pressure $\sim 2.7 \times 10^{-7}$ Torr). The lift-off process was performed for $15 \mathrm{~min}$ by immersing the samples in acetone. Finally, the samples were plunged in remover PG for $120 \mathrm{~min}$ at $70{ }^{\circ} \mathrm{C}$ heat. The SEM images shown in the manuscript were obtained using JEOL 7000 machine.

To characterize the multimetallic specimens and extract the plasmon response of multipixel arrays at room-temperature, a millimeter wave backward wave oscillator (BWO) setup combined with frequency multiplier (Microtech Instruments, Inc.) and broadband pyroelectric detector (Gentec Electro Optics Inc.) was used. The spectral range of the incident radiation was between $100 \mathrm{GHz}$ and $1.5 \mathrm{THz}$. On the other hand, to predict the plasmonic response of the structure, we carried out numerical analysis, performed using FDTD method. The bimetallic resonators on top of a semi-infinite highresistive silicon substrate were simulated using the refractive index of $\sim 3.64+i 0.0001$ for $\mathrm{Si}$ at $\mathrm{THz}$ spectrum [31]. The dielectric responses $\mathrm{Ti}$ and Fe were taken from empirically defined Palik values. Perfectly matched layers were used as boundaries, and the light source was a linear plane wave and to achieve precise results, the grid sizes in all axes were set to $25 \mathrm{~nm}$. 


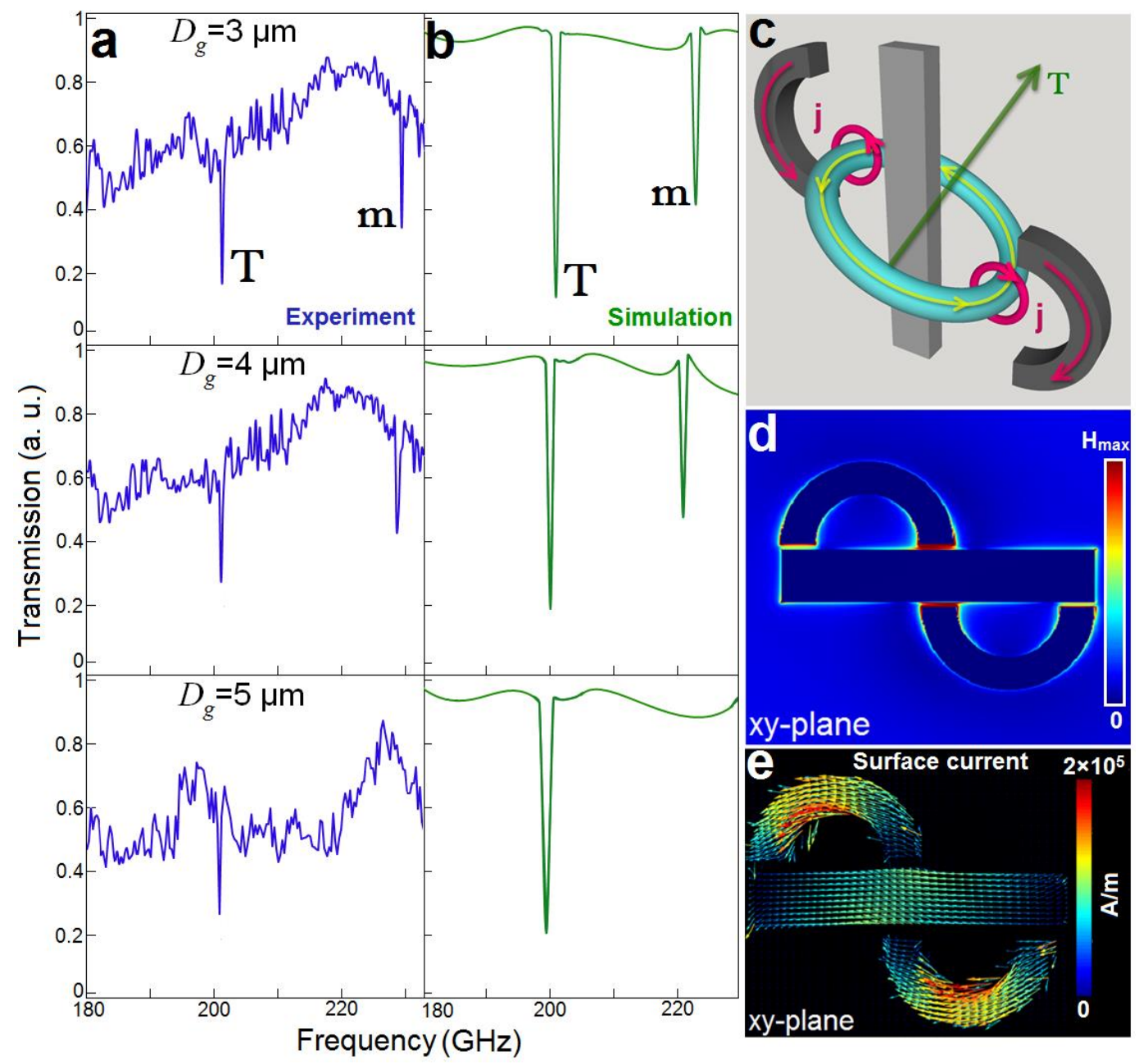

Figure 5.3. a), b) Experimentally and numerically defined transmission amplitudes for the plasmonic metamolecule with different gap distances, respectively. c) An artistic illustration for the formation of toroidal moment and circulation of closed-loop current. d), e) The local H-field map for the localization of plasmons at the toroidal frequency, and surface current density plot for the magnetic current oscillation across the antenna.

By launching the $\mathrm{THz}$ wave in the $[-z]$ direction, the excited local modes lead to formation of circular magnetic fields in the central zone of the peripheral curved structures. The experimental and numerical transmission spectrum for the plasmonic metamaterial are in consistent and depicted for the unit cells with three different gaps in Figs. 5.3a and 5.3b, respectively, under $y$-polarized beam illumination. In all three regimes, a significant toroidal dipole is excited around $\approx 200 \mathrm{GHz}$, while the intensity and 
linewidth of the optically induced lineshapes are slightly reduced by increasing the opening distance between central and peripheral resonators. This results in dramatic suppression of the electric dipole moment by the excited magnetic and toroidal resonances [18]. Figure 5.3c artistically demonstrates formation of a head-to-tail configuration of a magnetic moment as a toroidal dipolar moment $(\mathbf{T})$ at the center of the unit cell created by the currents $(\mathbf{j})$ on the surface of a torus along the circular meridian. The arrows show the current flux direction and magnetic moment $(\mathbf{m})$ oscillation as a close-loop arrangement inside the profile. Obviously, the head-to-tail configuration is formed with $90^{\circ}$ angle to the central block due to asymmetric geometry of metamolecule. Fundamentally, the antiparallel magnetic moments in peripheral resonators causes formation the toroidal moment, oscillating around the central resonator. The experimental and numerical quality factors for the toroidal dipole for the metamolecule with $D_{g}=3 \mu \mathrm{m}$ are estimated as 18 and 20 , respectively. It should be noted that a distinct resonant mode is observed in simulated and measured transmission spectrum for $D_{g}=3 \mu \mathrm{m}$ and $4 \mu \mathrm{m}$ around $\approx 220 \mathrm{GHz}$ correlating with the rotating magnetic momentum (m) arising from peripheral resonators that are not the case of interest here because they have a negligible influence on the polarization-dependency of the device and can be observed under both $x$ and $y$-polarized beam exposures. As it is obvious in the transmission spectra, there is an amplitude difference between the induced toroidal moments in numerical analysis and experimental measurements due to air humidity and the presence of impurities in the BWO setup environment. The local H-field intensity is illustrated in Fig. 5.3d for the toroidal dipole frequency, showing localization of magnetic momentum at the position of head-to-tail current oscillation. Finally, Fig. 4.3e illustrates the surface current plot for the 

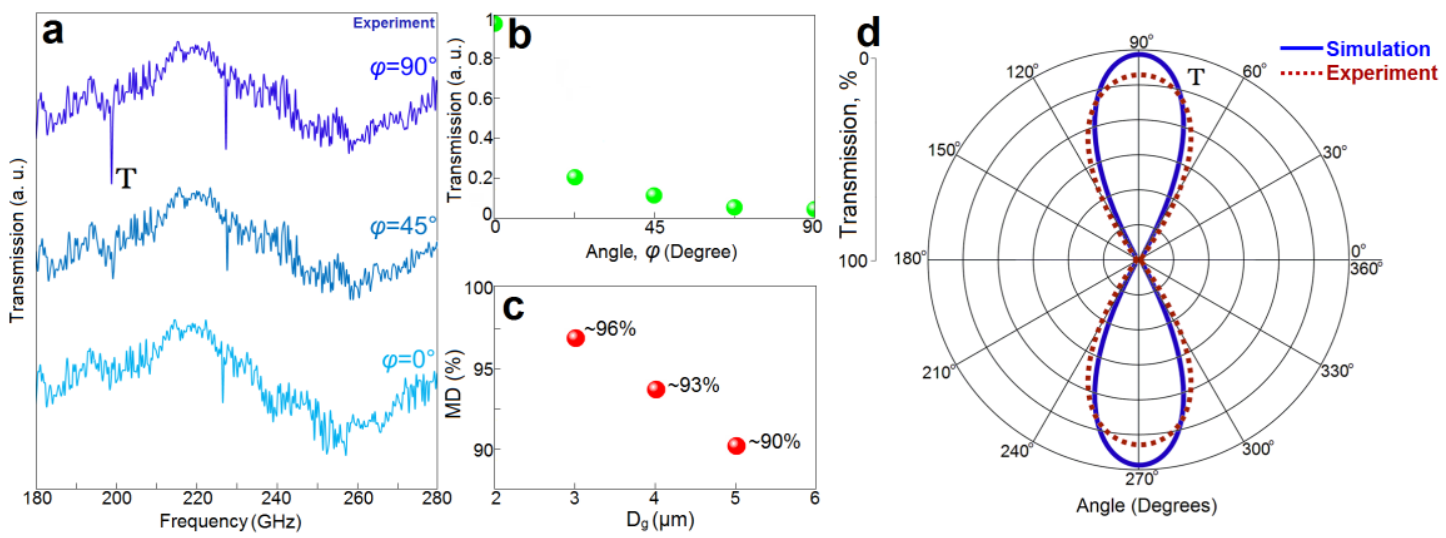

Figure 5.4. a) Experimentally measured normalized transmission amplitude for both toroidal and magnetic responses of the plasmonic unit cell under different magnetic polarization angles $0^{\circ} \leq \varphi \leq 90^{\circ}$. b) Toroidal response of the unit cell as a function of incident $\mathrm{THz}$ radiation magnetic component angle. c) The MD percentage as a function of $D_{g}$, showing the highest value around $\sim 96 \%$. d) The polar plot for both experimentally and numerically obtained transmission spectra at the position of the toroidal mode.

toroidal metamolecule, verifying antiparallel oscillation of current in the peripheral resonators.

By taking advantage of the inherent asymmetry of the plasmonic structure, we demonstrate an efficient polarization-dependency and fast plasmonic response for the toroidal structure. This would help us to develop a promising toroidal platform for photonic switching applications with ultrafast spectral response. By choosing the structures with the highest quality factor from the prior investigations, we analyze the behavior of a sample unit cell under varying $\mathrm{THz}$ beam polarization states. In Fig 5.4a, we plotted the experimentally measured normalized transmission spectra for the unit cell with the following geometries: $D_{g}=3 \mu \mathrm{m}$, with $L=240 \mu \mathrm{m}, R=50 \mu \mathrm{m}, W_{l}=30 \mu \mathrm{m}$, and $W_{2}=40 \mu \mathrm{m}$, to achieve the highest possible quality factor. In principle, for the incident magnetic beam in the longitudinal polarization limit $\left(\varphi=90^{\circ}\right)$ parallel to the central block $(\mathrm{H} \|)$, the same toroidal dipolar dip is induced with high quality around $\approx 200 \mathrm{GHz}$ and the beam transmissivity is low and around $\sim 19 \%$. Slightly rotating the angle of the incidence 
to $\varphi=45^{\circ}$, we observed a drastic decay in the toroidal resonant mode dip. Eventually, for $\varphi=0^{\circ}$, where the incident magnetic component entirely transverse $(\mathrm{H} \perp)$ to the central block, the toroidal dip is eliminated and the plasmonic metasurface acts as a transparent medium at this frequency. As a result of this rotation, the toroidal resonance characteristics disappeared. To underline the physics behind the process of toroidal mode removal, we look into the incident electric component $(\mathrm{E} \|)$ as well. For $\varphi=0^{\circ}$, due to the asymmetric geometry of the plasmonic unit cell, the incident electric component becomes parallel $(\mathrm{E} \|)$ to the central block and offset gaps. In this regime, the electric field becomes dominant and the required head-to-tail magnetic current close-loop cannot be formed around the central block of the multiplex unit cell. Interestingly, however, a distinct magnetic dipolar momentum around $\approx 225 \mathrm{THz}$ still remained due to excitation of dipolar magnetic resonances supporting by peripheral curves via transverse incident magnetic beam. Moreover, the transmission spectra (for the toroidal response) as a function of the magnetic component of the incident beam angle $(\varphi)$ is plotted in Fig. 5.4b. Such a huge dependency of toroidal minimum on the incidence angle can be exploited for fast and efficient on/off routing and filtering purposes. As a key parameter, we also computed the corresponding MD for the proposed metasurface as a function of gap distance between resonators [32-34], as shown in Fig. 5.4c. Here, the best MD percentage is estimated around $\sim 96 \%$ for a resonator with the gap size of $D_{g}=3 \mu \mathrm{m}$. The plotted diagram shows the strong dependency of the toroidal dipolar mode and subsequently MD to both geometrical and polarization distributions. Ultimately, to verify this claim, we plotted the polar plan for the analyzed unit cell for the transmission spectra in Fig. 5.4d. Sustaining an ultrasharp toroidal mode by a bimetallic metamolecule allowed us to 
develop a polarization-dependent and high quality structure for advanced $\mathrm{THz}$ applications such as switching and filtering.

\subsection{Tunable Toroidal Resonances}

The radiation patterns produced by the examined resonant modes in pearlier sections and in the current chapter (i.e. toroidal and Fano lineshapes) cannot efficiently couple to free space, hence, we expect strong localization of EM field squeezed in a single point at the position of these resonances. Although both Fano and toroidal modes can be exploited for development of advanced optical devices, they largely suffer from the lack of spectral tunability $[35,36]$. Despite of exquisite features of the induced resonances in regular resonators, however, lack of geometrical flexibility in either planar or 3D unit cells limits possessing active control over the excited modes. Designing a unit cell with the ability of supporting different resonances without physical changes in the design would allow for tailoring a metasurface with exquisite controllability over the spectral response. Such an advantage can be obtained by using heat, voltage, and/or light-intensity dependent materials in the geometry of the proposed design.

As it is mentioned in chapter 4, PCMs are chalcogenide alloys, have been employed for fabricating efficient non-volatile and rewritable data storages. The advantage of switching between entirely opposite phases of amorphous and crystalline states was a concept of interest to develop various nanophotonic devices [37-39]. Similar to the previous analysis, we use GST as one of the most common PCMs, which shows reasonably fast and large refractive index variance between amorphous and crystalline phases in the NIR [38]. For instance at the C-band (telecommunication bandwidth, $\lambda \sim 1550 \mathrm{~nm})$, the a-GST and c-GST reflect have the refractive indices: $n_{\mathrm{a}-}$ 

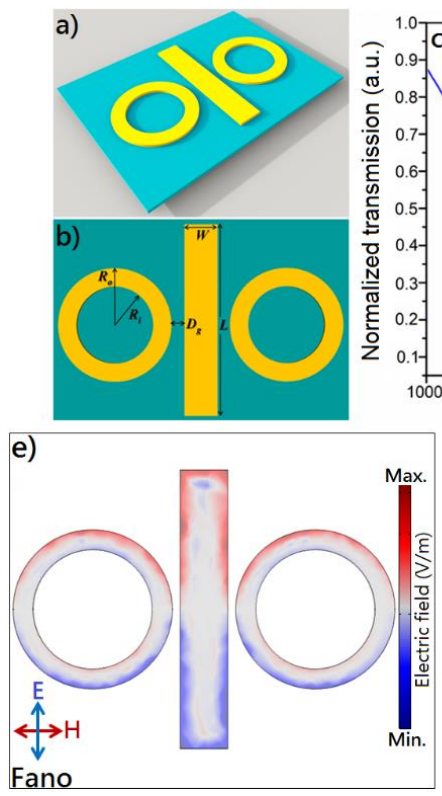
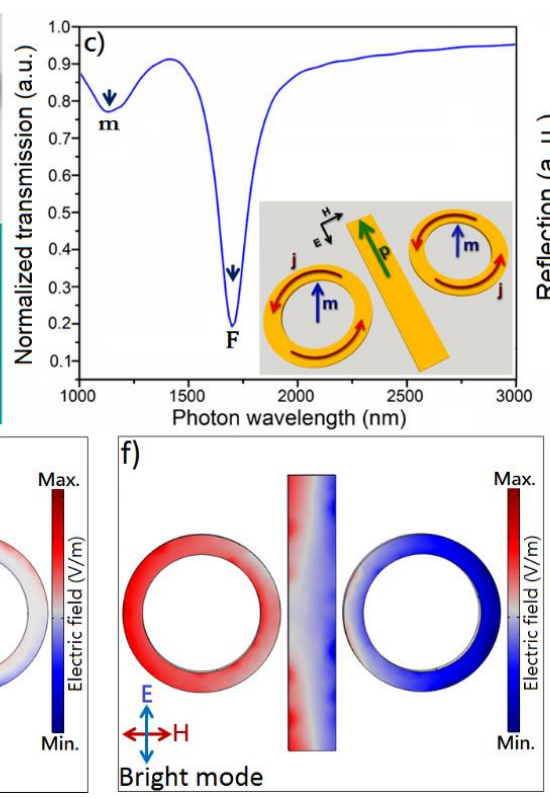
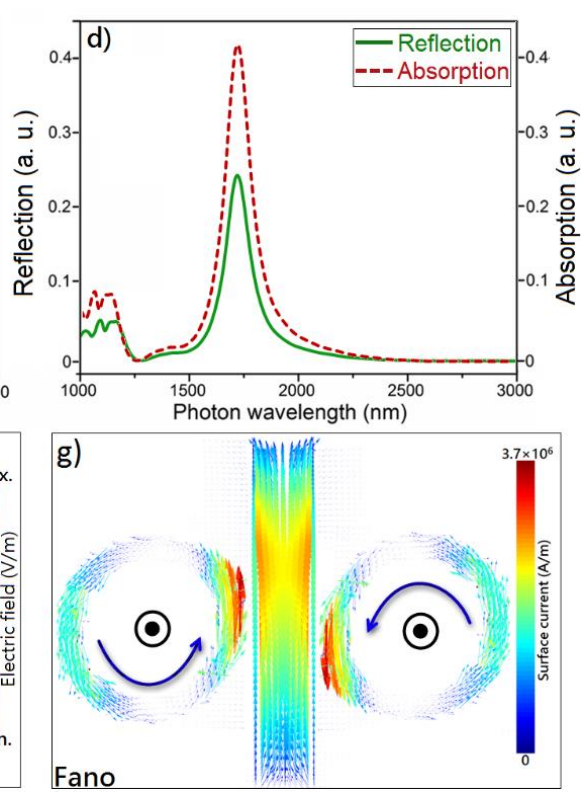

Figure 5.5. Fano resonant unit cell with the geometrical dimensions as follows: $\left(\boldsymbol{R}_{o} / \boldsymbol{R}_{i} / \boldsymbol{D}_{g} / \boldsymbol{W} / \boldsymbol{L}\right)=(\mathbf{9 5} / \mathbf{6 5} / \mathbf{1 0} / \mathbf{7 0} / \mathbf{3 2 5}) \mathbf{n m}$. a) Schematic and b) top-view profiles for the plasmonic unit cell. c) Normalized amplitude transmission spectra for the Fano resonant structure. The inset is a rendering for the coherent oscillation of charges. d) Normalized absorption and reflection spectra for the proposed unit cell. e), f) The local E-field maps for the plasmon resonance excitation at the Fano dip and bright mode positions, respectively. g) The surface current of resonator at narrow Fano dip wavelength.

$\operatorname{GST}_{18.89+i 2.175}(\operatorname{Im}(n) / \operatorname{Re}(n)<0.1) \quad$ and $\quad n_{\mathrm{c}-\mathrm{GST}} \sim 48.61+i 18.68 \quad(\operatorname{Im}(n) / \operatorname{Re}(n)<0.3)$, respectively $[34,39]$.

To address the limited tunability of plasmonic moments, in this section, we describe a novel metallodielectric plasmonic unit cell consisting of a blend of metallic and PCM parts to support both Fano and toroidal dipolar moment along the NIR at different states of the employed PCM. Taking advantage of the switching between amorphous and crystalline phases of the PCM in the designed plasmonic resonator, we demonstrated the excitation of distinctly different resonant modes in the unit cell without any geometrical variations. Using electromagnetic computations, we show that a careful combination of metallic and GST parts helps to have active control over the interplay between Fano and 
toroidal dipolar resonances by varying the magnetic resonance circulation direction. We also analyzed the NIR switching mechanism of the proposed structure to develop practical telecommunication devices.

The schematic and top-view images for the firstly analyzed symmetric plasmonic unit cell is shown in Figs. 5.5a and 5.5b, respectively, with geometrical details inside. The required materials data and EM computation techniques are explained in the Methods section. The proposed nanoplasmonic system composed of two nanorings located apart with a rectangular bar in between. Figure 5.5c illustrates the normalized transmission amplitude for the plasmonic resonator showing formation of an antisymmetric and pronounced Fano resonant (F) mode around $\sim 1700 \mathrm{~nm}$. According to the plasmon hybridization theory [40], two nanorings located close to each other with a rectangle resonator in between as a bright electric dipole supporter, directly interact and couple to free space. Besides, as the near-field components, the surrounding nanorings sustain dark magnetic dipoles (m), which couple efficiently to the bright electric mode [41,42]. The inset is the rendering for the magnetic moment rotation and direction of the excited charges. The geometrical dimensions are specified in the figure legend. Here, the magnetic moments in both peripheral rings oscillate in the coherently and destructively couple to the bright mode from the central bar and leads to formation of a distinct Fano minimum. The normalized absorption and reflection spectra are exhibited in Fig. 5.5d, consistent with the optically driven Fano dip. The local E-field maps for the excited plasmons at the Fano dip and bright dipolar mode positions are plotted as snapshots in Figs. 5.5e and 5.5f. Figure 5.5g exhibits the surface current density in a vectorial plot at 

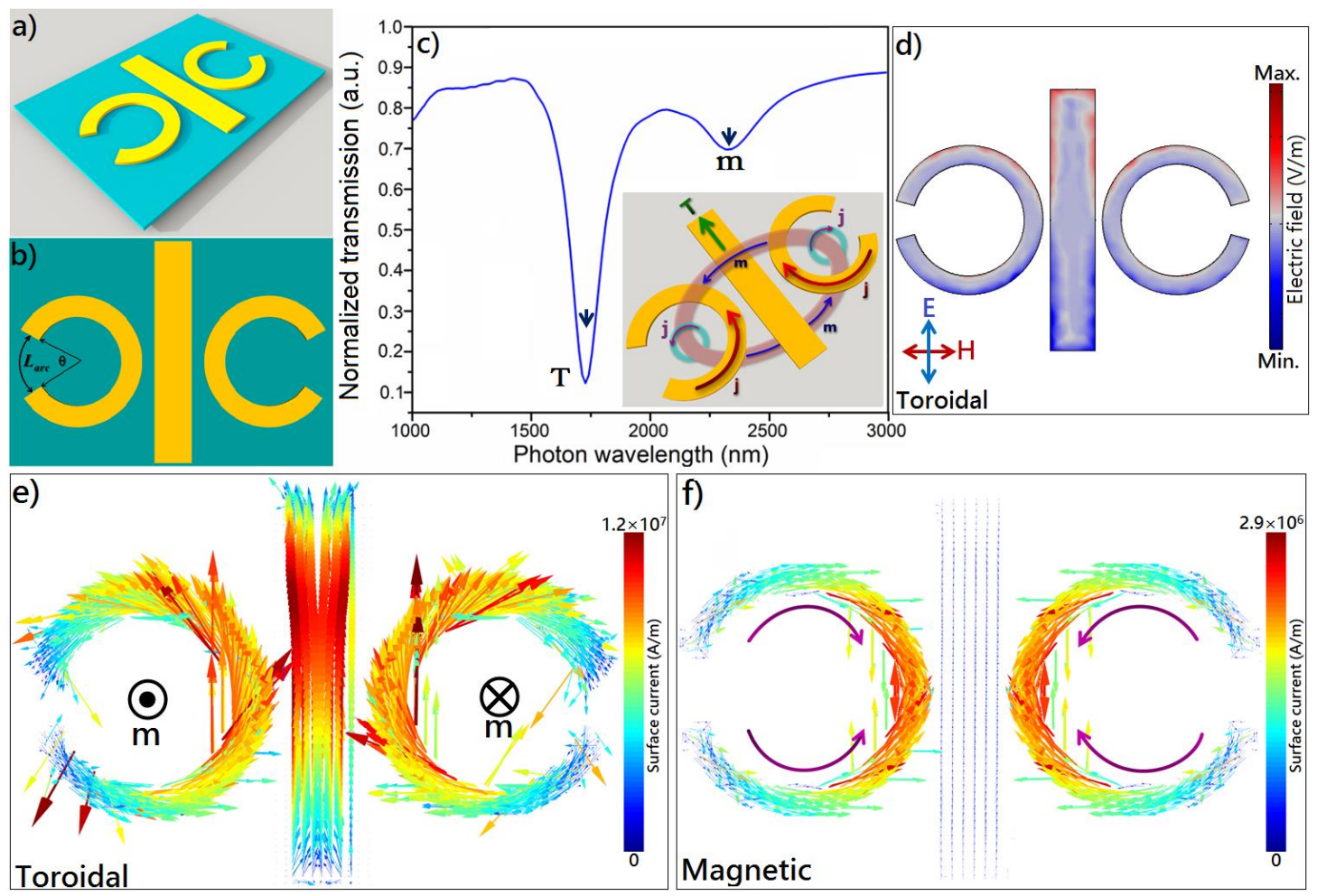

Figure 5.6. Toroidal resonant unit cell. a) Schematic and b) top-view profiles for the plasmonic unit cell. c) Normalized amplitude transmission spectra for the toroidal structure. The inset is a rendering for the opposite oscillation of magnetic moments, showing formation of a closed-loop current. d) The local E-field maps for the plasmon resonance excitation at the toroidal dipole position. e) and f) The surface current of resonator at toroidal and magnetic dipole wavelengths, respectively.

the Fano dip wavelength, confirming that magnetic moments in surrounding nanorings are oscillating in the same direction.

On the other hand, previous studies have shown that breaking the symmetry of plasmonic nanostructures enhances the dark mode and alters the width and position of the Fano resonance [42-44]. Conversely, in the present system, breaking the symmetry leads to significant alterations in the magnetic current direction, resulting in unveiling of significant toroidal momentum. Figures 5.6a and 5.6b illustrate the schematic and topview pictures, respectively, for the new unit cell with the broken rings. By removing a certain part of the nanorings with a given arc length (defined by: $L_{a r c}=2 \pi R_{o}\left(\theta / 360^{\circ}\right)$ ), 
we prevent coherent rotation of magnetic moment in both rings, whereas the capacitive gaps play fundamental role in the elimination of Fano minimum. Assuming the metasurface consists of infinite number of the proposed 2D unit cells in both $x$ - and $y$ axes, the corresponding projection of toroidal dipolar moment normal to the $z$-axis $(k$ direction) can be defined by: $\mathbf{T}_{\|}=T_{x} \hat{\mathbf{x}}+T_{y} \hat{\mathbf{y}}$ or in general: $\mathbf{T}_{\|}=\mathbf{T}-(\mathbf{T} \cdot \hat{\mathbf{R}}) \hat{\mathbf{R}}$, as discussed comprehensively in the previous section of this chapter. Using Eq. 5.36, the reflected electric field from the metasurface for toroidal dipolar momentum as dominant component can be written in a brief version of Eq. 5.41 as below:

$$
\overrightarrow{\mathbf{E}}_{\mathrm{s}}=\left(\frac{\mu_{0} c^{2}}{2 \Delta^{2}}\right) \exp (-i k r)\left[-i k \overrightarrow{\mathbf{p}}_{\|}+i k \hat{\mathbf{r}}\left(\overrightarrow{\mathbf{m}}_{\|}-\frac{k^{2} \overrightarrow{\mathbf{m}}_{\|}^{(1)}}{10}\right)-k^{2}\left(\overrightarrow{\mathbf{T}}_{\|}-\frac{k^{2} \mathbf{T}_{\|}^{(1)}}{10}\right)\right]
$$

In the equation above, only the dominant term, which is the dipolar toroid term is kept and multipolar terms for toroidal moment are ignored. Figure 5.6c shows the transmission spectra for the modified structure, where the Fano dip disappeared and a new narrow resonance appeared around $\sim 1775 \mathrm{~nm}$. Further, a magnetic dipole moment $(\mathbf{m})$ is excited at lower energies around $\sim 2350 \mathrm{~nm}$. The transmitted electric field from the nanostructure is defined by adding up the incident beam and the scattered electric field from Eq. 5.44. The inset shows the formation of a closed-loop current correlating with the toroidal moment due to the incoherent rotation of the magnetic moments in surrounding broken rings graphically. The local E-field intensity map in Figure 5.6d illustrates excitation of magnetic resonances at the toroidal mode position using FEM analyses. In order to verify formation of toroidal resonance, we plotted the surface current vectorial plot as shown in Figs. 5.6e and 5.6f. At $1675 \mathrm{~nm}$, the charges oscillate in opposite directions in the 


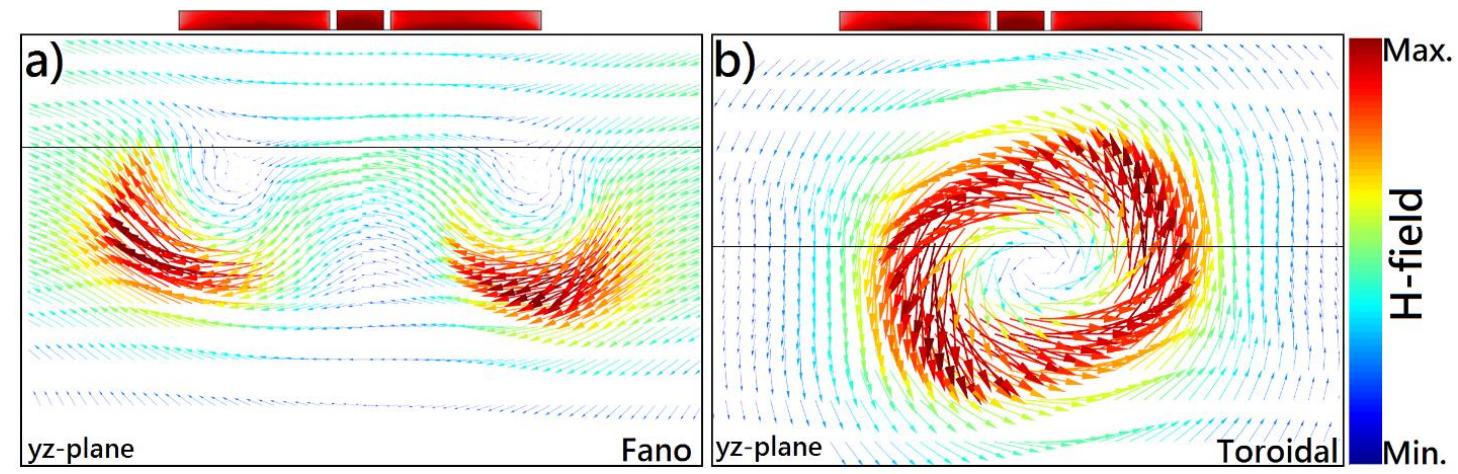

Figure 5.7. The $y z$-plane for the magnetic field $(|\mathrm{H}|$-field $)$ excitation and direction across the unit cell for a) Fano dip and b) toroidal dipole.

surrounding broken nanorings, hence, the magnetic moments do not couple to the bright dipolar mode arising from the central resonator. The magnetic vectorial charge plot demonstrates oscillation of charges in a head-to-tail configuration (toroidal magnetic moment, T) in each isolated nanoring, and leading to formation of a big close-loop arrangement. This magnetic closed-loop current is formed due to opposite rotation of magnetic dipolar momenta in the nanorings stimulated by the capacitive gap regions. Noticing in Fig. 5.6f, at the magnetic dipole moment position, the excited magnetic fields in both rings oscillate in a head-to-head configuration. To verify this claim, we plotted the $y z$-plane of magnetic (H-field) for the plasmonic structure with and without arc section at both Fano and toroidal momenta wavelengths as shown in Figures 5.7a and 5.7b, respectively. Obviously, for the Fano dip position, the charges oscillates coherently and couple to the bright mode (Figure 5.7a). On the other hand, for the toroidal mode, the charges oscillate in a head-to-tail arrangement across the unit cell (Figure 54.7b). 

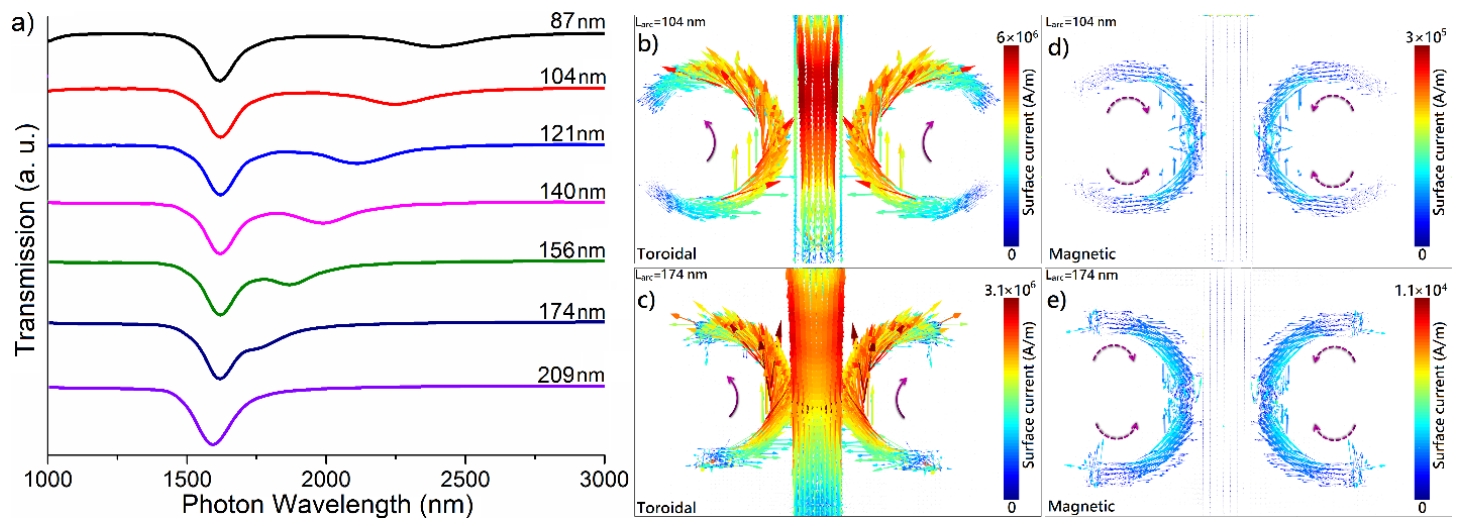

Figure 5.8. (a) Transmission spectra for the toroidal resonator for samples having different arc lengths $\left(L_{a r c}\right)$. (b) and (c) The surface current of resonator at toroidal dipole position for two different arc lengths, 104 and $174 \mathrm{~nm}$, respectively. (d) and (e) The surface current of resonator at magnetic dipole moment for two different arc lengths, 104 and $174 \mathrm{~nm}$, respectively.

Focusing on the toroidal moment as the unique response of the structure, the length of the arc plays important role in the behavior and position of both toroidal dipole and magnetic modes. To show the effect of arc length, we studied the effect of this parameter on the excited modes for $87 \mathrm{~nm}<L_{a r c}<209 \mathrm{~nm}$, as shown in Figure 5.8a. Increasing the arc length (gap distance) leads to a dramatic blue-shift in the position of magnetic dipole to the shorter spectra and finally disappeared and dampened for the largest examined arc length $\left(L_{a r c}=209 \mathrm{~nm}\right)$. On the other hand, the toroidal dipole slightly blue-shifted including an enhancement in its depth. Such a spectral response can be better understood by plotting the current density flowing on the surface of the surrounding nanorings and central block for toroidal dipole and magnetic modes as depicted in Figures 5.8b-5.8e for $L_{a r c}=104 \mathrm{~nm}$ and $174 \mathrm{~nm}$. At the toroidal dipole wavelength, the direction and intensity were not affected significantly by the morphological variations (see Figures $5.8 \mathrm{~b}$ and 5.8c). In contrast to the Fano resonance, the negligible dependence of the toroidal resonances on major geometrical variations and antisymmetric properties is verified here. On the other hand, by increasing the length of the arc, the head-to-head magnetic 

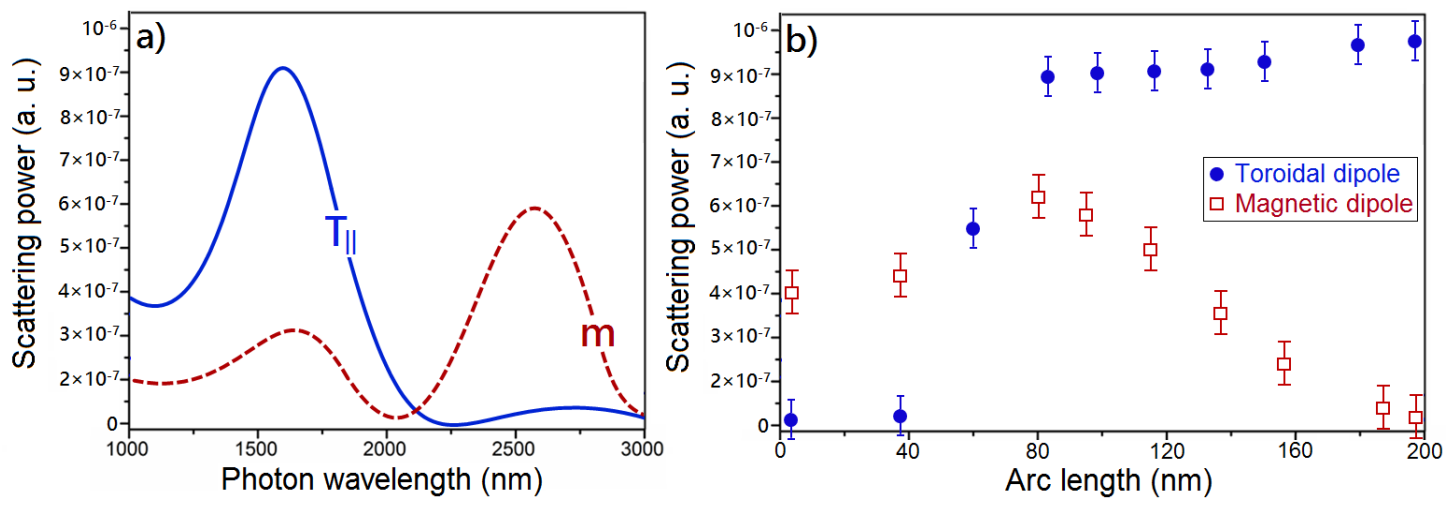

Figure 5.9. a) Scattering powers of toroidal and magnetic moments $\left(L_{a r c}\right)$. (b) The scattering power as a function of arc length variations for toroidal and magnetic moments.

oscillation decayed due to imperfect rotation of the magnetic dipoles (see Figures5.8d and 5.8e).

Comparing Figure 5.8a and surface currents flow for two specific arc lengths, the results for the toroidal and magnetic resonance intensities are consistent. The effect of arc length can be further investigated by quantifying the far-field scattering power or intensity for toroidal and magnetic moments using Eqs. 5.42b and 5.42c. Figure 5.9a exhibits the quantified scattering intensity profiles for both projected toroidal and magnetic dipole resonant moments for $L_{a r c}=87 \mathrm{~nm}$. The appeared distinct peaks are correlated with the targeted resonant modes with dominant scattering power for projected toroidal and magnetic moments normal to the incident beam direction ( $z$-axis). Fig. 5.9b illustrates the scattering power profile as a function of varying arc length, showing the intensity of magnetic moment decayed drastically, however, the intensity of toroidal dipole remained nearly constant with a slight blue-shift to the higher energies. The provided numerical calculations for the proposed plasmonic unit cell show formation of strong and pronounced Fano and toroidal resonant modes along the NIR using a fully metallic unit cell in two different regimes. Although, supporting both Fano and toroidal 
resonance modes on the same structure without geometrical modifications helps to design advanced nanoscale devices, the currently analyzed metasurface still lacks tunability on the plasmonic response similar to the analogous nano and microstructures in previous works $[14,46-48]$. To address this limitation, in continue, we show that how we can efficiently control the interplay between Fano and toroidal resonances without morphological variations. To this end, we introduce a PCM (here GST) into the gap areas (arcs) with the ability to support different amorphous and crystalline states at different temperatures. This approach yields an active switching between two distinctly different resonances. Figure 5.10a illustrates an artistic picture for the metallic unit cell with the presence of GST in the arc-shape gap areas, resembling a metallodielectric plasmonic nanostructure. By changing the length of the arc filled with GST substance, we computed the spectral response in Figs. 5.10b and 5.10c. For the amorphous phase (a-GST), the arc acts as a dielectric material, and due to dominancy of capacitive coupling, we expect formation of toroidal moment as well as a weak magnetic moment at lower energies (Fig. 5.10b). By increasing the arc length from $87 \mathrm{~nm}$ to $174 \mathrm{~nm}$, we detected a minor decay in the toroidal dipole moment, while the magnetic dipole damped significantly and blueshifted similar to the previous analyses in this context. On the other hand, by applying the continuous beam to change the phase of GST sections to the crystalline state (see methods), the arc behaves as a semi-metallic and conductive material, leading to formation of a Fano dip in the spectral response (Fig. 5.10c). In addition, an intense magnetic dipole appeared at the left side of the Fano dip for shorter arc lengths, led to formation of a broad electromagnetically induced transparency (EIT)-like response around $\sim 1400 \mathrm{~nm}$. However, increasing the arc length causes to blue-shift in the position 


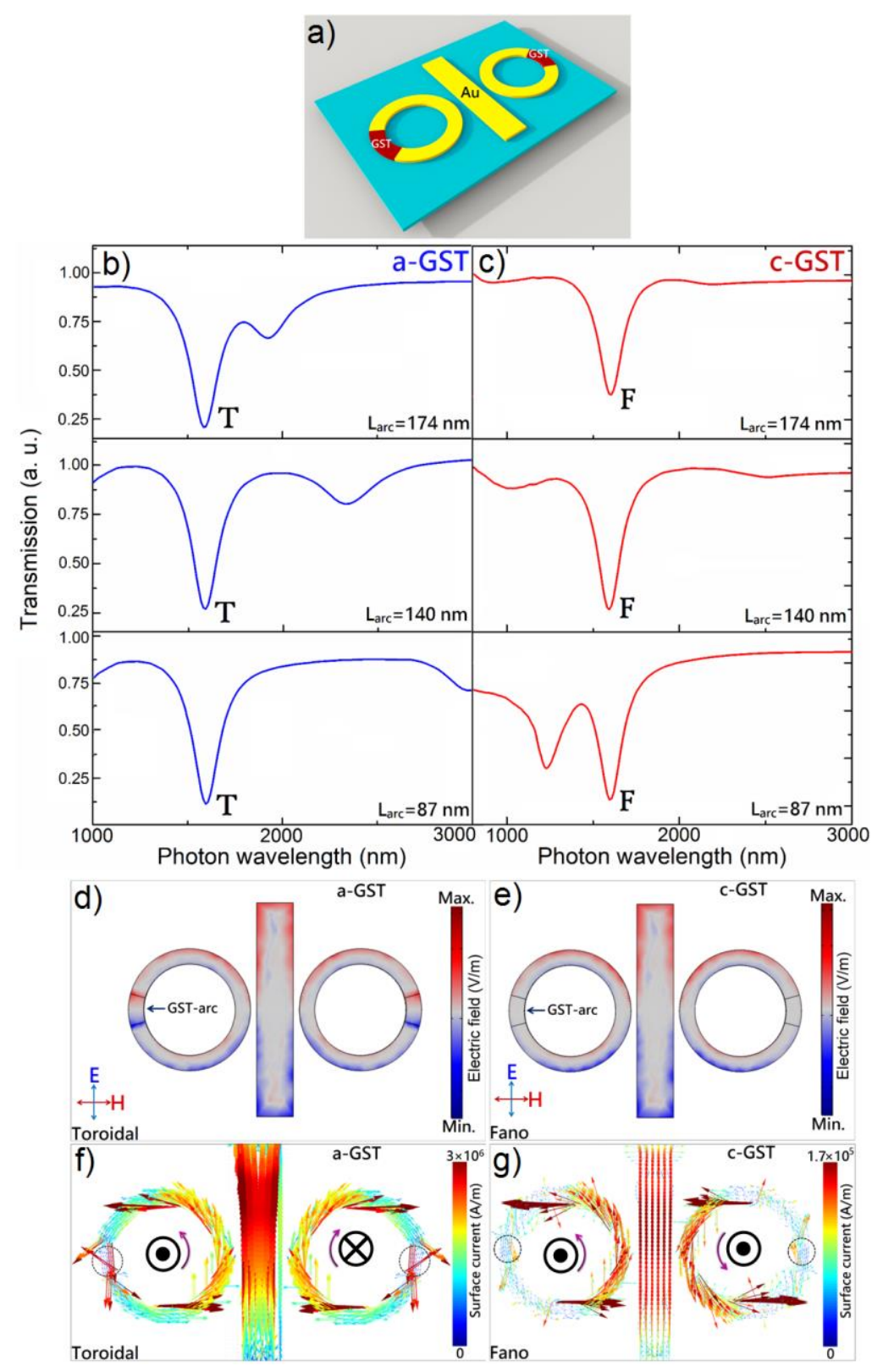

Figure 5.10. a) Schematic for the metallodielectric unit cell with GST arcs. b) and c) The transmission spectra for the arc length variations in a-GST and c-GST states of arcs, respectively. d) and e) The E-field maps for the metallodielectric unit cell in c-GST and aGST phases of arcs, respectively at the Fano and toroidal wavelengths. f) and g) The surface current density for the c-GST and a-GST states of arcs.

of magnetic dip and finally vanishing, while the Fano minimum approximately remains unchanged. The E-field maps for the plasmonic Fano and toroidal modes are illustrated in

Figs. 5.10d and 5.10e (for $L_{a r c}=87 \mathrm{~nm}$ ). Accordingly, the c-GST arcs in both rings act as a 

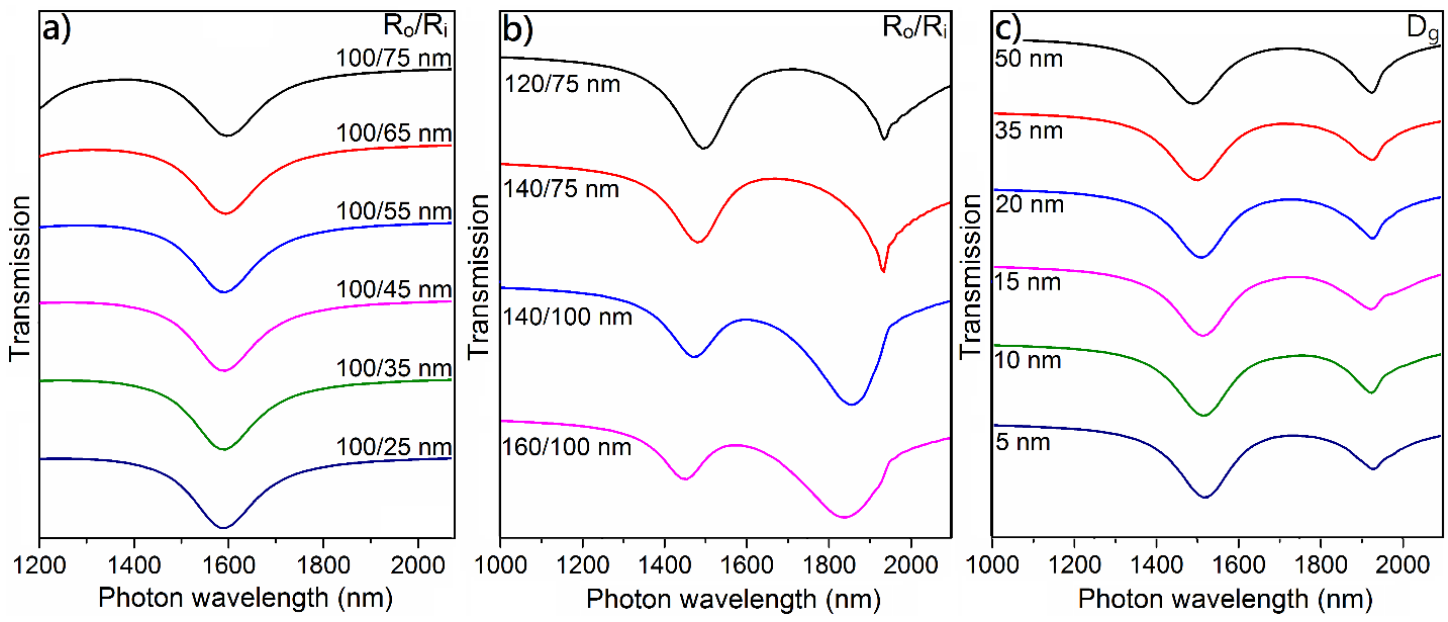

Figure 5.11. Transmission spectra for the toroidal dipole for (a) inner radius, (b) outer radius, and (c) gap distance variations.

conductive substance, leading to coherent excitation of charges and formation of asymmetric Fano dip (Fig. 5.10d). On the other hand, for the a-GST, due to dielectric behavior of GST arc, the charges oscillate oppositely, whereas the effect of arc section is distinct in Fig. 5.10e. Figures 5.10f and 5.10g compare the surface current density for the metallodielectric unit cells with GST substance in two opposite phases, which are consistent with the earlier studies in this work. The dashed circles at the GST position, exhibits how charges intensely couple in capacitive a-GST arc and easily shuttle in cGST regimes. In continue, we carry out series of computations to enhance the quality factor of the induced Fano dip and toroidal moment by concentrating on the geometrical modifications. Due to different natures of Fano and toroidal modes, we expect strong dependency of each resonance to specific geometrical components. Starting from metallodielectric unit cell with a-GST arc, which can sustain pronounced toroidal dipole, we analyzed the spectral behavior as shown in Fig. 5.11. The inner radius $\left(R_{i}\right)$ variations in the range of $25 \mathrm{~nm}$ to $75 \mathrm{~nm}$, (see figure legend) for the satellite nanorings, while the other geometries are kept fixed, did not affect the toroidal dipole considerably. A minor 
blue-shift in the position of toroidal mode to the shorter wavelengths (Fig. 5.11a) is observed. In addition, it is noteworthy that the magnetic dipole $(\mathrm{m})$ is located at longer spectra beyond $2800 \mathrm{~nm}$ (not shown). On the other hand, a dramatic decay in the toroidal dipole is observed by increasing the outer radius $\left(R_{o}\right)$ of the particles. Interestingly, the magnetic dipole remarkably red-shifted to the higher energies including a continuous broadening in the lineshape (Fig. 5.11b). Finally, we examined the effect of gap distance $\left(D_{g}\right)$ between the central resonator and the surrounding nanorings on the plasmonic response of toroidal unit cell (Fig. 5.11c). By changing the gap distance between central bar and the neighbor nanorings homogenously in the range of $5 \mathrm{~nm}$ to $50 \mathrm{~nm}$, we observe that the toroidal moment is not affected by the capacitive gap size due to missing coupling between the dipolar electric mode excited from central bar and the magnetic moment from the peripheral rings. Noticing in the transmission spectra for gap variations, a subtle red-shift in the position of the toroidal dipole is observed including a decay in the amplitude. One should note that due to negligible influence of the central resonator on the toroidal response, we did not consider the effect of this component. On the other hand, for the unit cell with the c-GST arc, the induced Fano resonance strongly depends on the gap spots and the central block. Figures 5.12a-5.12d exhibit the geometrical variations for the inner and outer radii, gap distance, and central resonator geometries, respectively. It is obvious that how Fano resonance is sensitive to the minor geometrical alterations, comparing to the toroidal dipole. Being almost fixed for the nanoring variations (Fig. 5.12a), a huge sensitivity for Fano dip is observed for the variations in the offset gap distance and central resonator. Figure 5.12b shows the red-shift in the position of Fano dip by increasing the gap size including a decay in the asymmetric dark mode. On the 

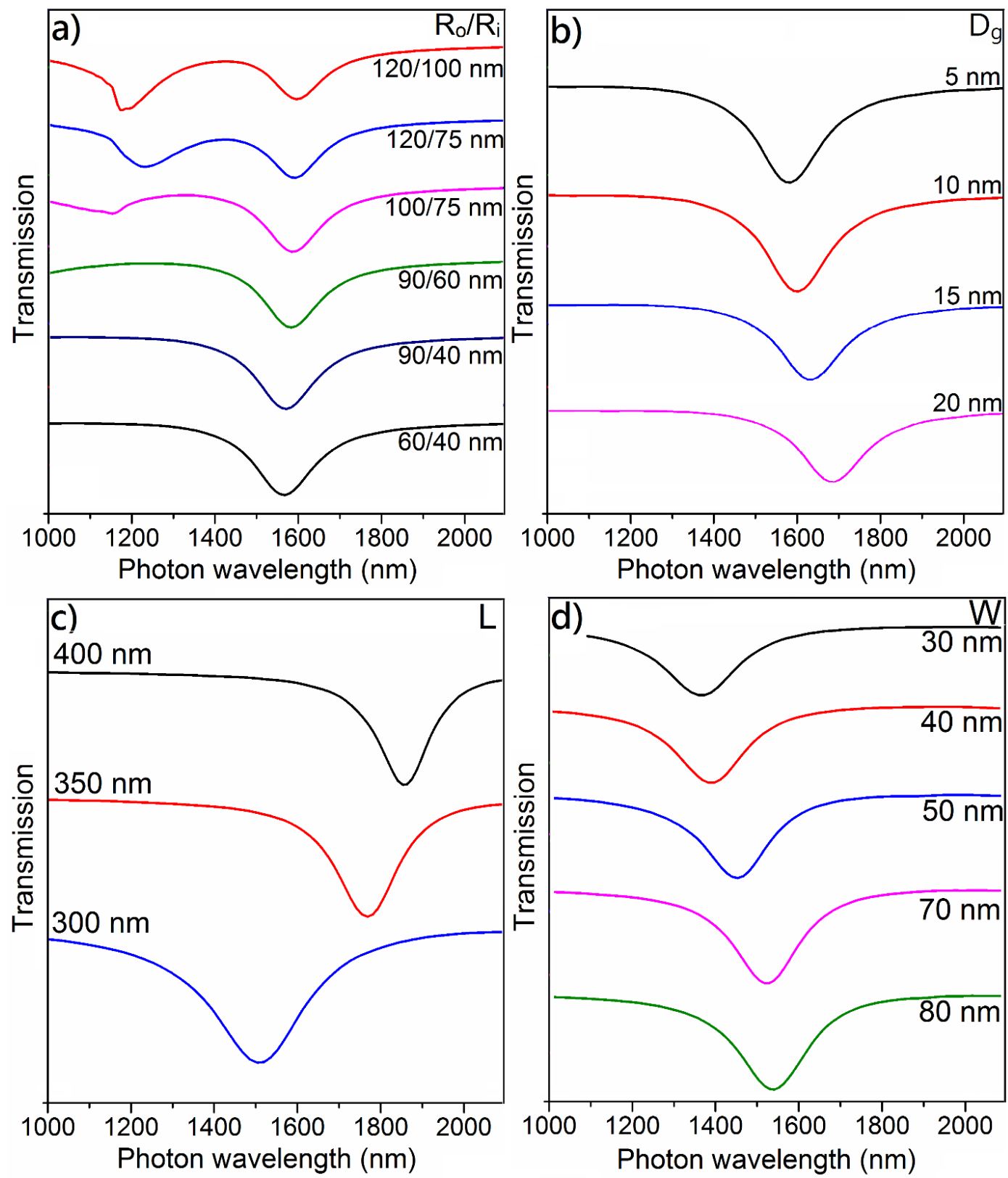

Figure 5.12. Transmission spectra for the Fano dip for (a) inner and other radii, (b) gap distance, (c) and (d) length and width of the central bar, respectively, variations.

other hand, minimizing the size of the middle bar (both $L$ and $W$ ), significantly blue-shifts the Fano dip to the shorter wavelengths (Figs. 5.12c and 5.12d). One should note that the magnetic moment disappeared or greatly suppressed due to these geometrical variations. By using the obtained data and careful selection of the dimensions for the unit cell 
geometries, we plotted the transmission amplitude for a potential telecommunication switch (see Fig. 5.13). The optimized geometries for the metallodielectric unit cell with the high quality toroidal dipole and asymmetric Fano dip are $\left(R_{o} / R_{i} / D_{g} / W / L\right)=(95 / 65 / 10 / 70 / 325) \mathrm{nm}$. The corresponding quality factors for toroidal dipole and Fano dip are quantified as 14.8 and 15.6, respectively. As shown in Fig. 5.13, the GST arc supports a toroidal resonance at $1550 \mathrm{~nm}$ (global telecommunication wavelength, Off-state) when it is in amorphous state. By applying an optical pulse and heating the structure, the GST arc switches to the crystalline phase, leading to formation of a Fano dip away from $1550 \mathrm{~nm}$ (On-state). The rapid switching between opposite states of GST helps to develop promising and efficient plasmonic devices for practical applications.

In this section, using PCM-mediated metallodielectric unit cells, we demonstrated fully reversible active control over the interplay between Fano and toroidal plasmonic resonant modes. For the purely metallic unit cell and complete nanorings, we observed excitation of asymmetric Fano resonant lineshape across the NIR. By removing arc-shape sections of the nanorings, we interfered in the direction of oscillation of magnetic modes and induced toroidal dipole resonance along the same domain. To provide reversible and fully controllable modulation of the excited plasmons and switching between Fano and toroidal moments, we introduced GST arcs as a thermally functional compound in the unit cell. Our numerical results confirm that switching the phase of GST arc between amorphous and crystalline regimes gives rise to variations in the magnetic moment oscillation in both nanorings. This study represents a useful and practical approach to the active tuning and controlling of the excited plasmonic resonances without morphological 


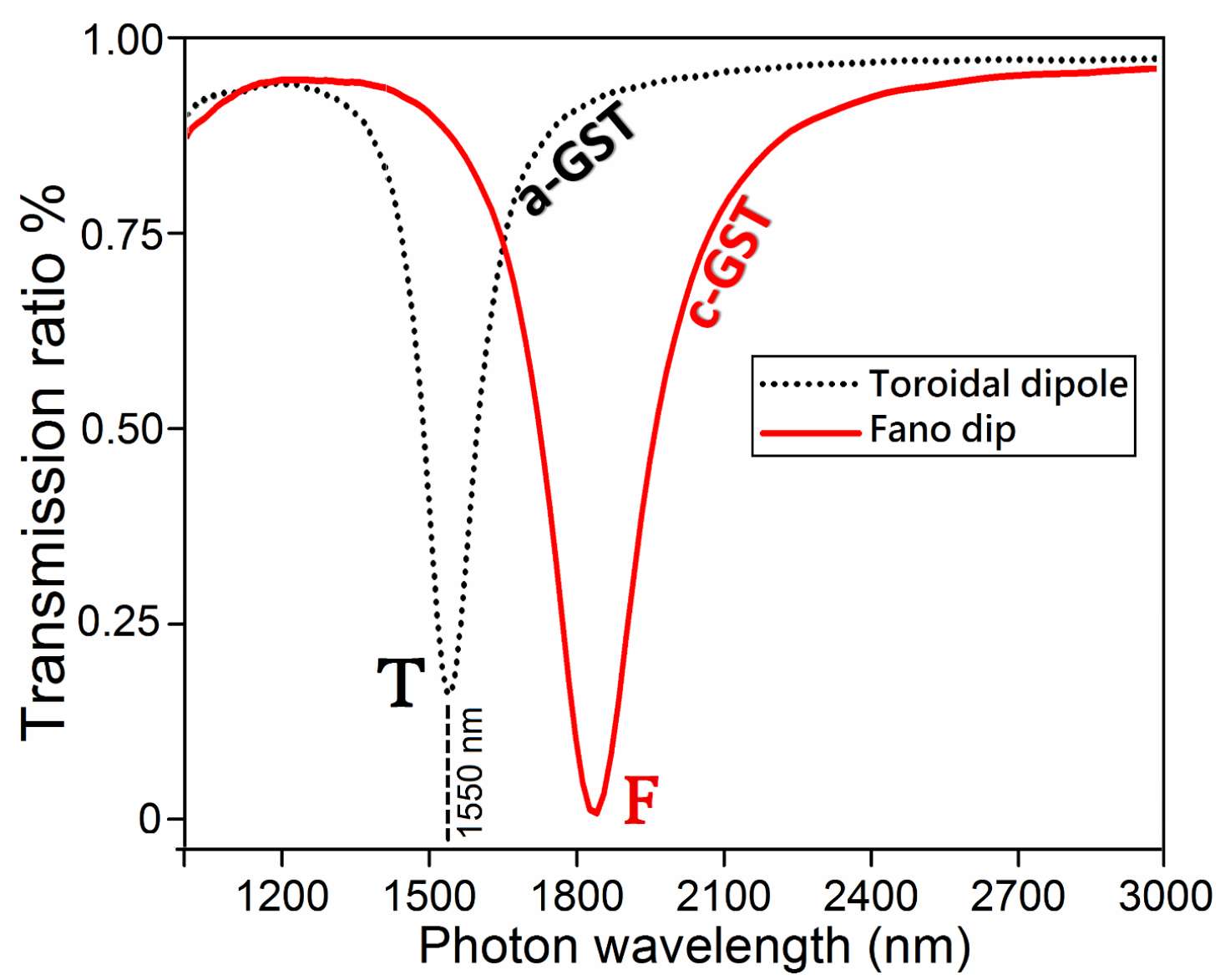

Figure 5.13. Transmission ratio for the GST-mediated metallodielectric unit cell in both a-GST and c-GST states, resembling an NIR plasmonic switch.

variations in the geometry of the unit cell. The expressed understanding in this section of chapter opens new avenues to tailor novel and advanced nanoplasmonic devices and also shows how strategic resonant moments can be tuned efficiently using functional materials in designing the meta-atoms and metamolecules.

\subsection{References}

1. Raab, R. E., \& De Lange, O. L. (2005). Multipole theory in electromagnetism: classical, quantum, and symmetry aspects, with applications. (Vol. 128). Oxford University Press.

2. Papasimakis, N., Fedotov, V. A., Savinov, V., Raybould, T. A., \& Zheludev, N. I. (2016). Electromagnetic toroidal excitations in matter and free space. Nature Materials, 15, 263-271. 
3. Kuznetsov, A. I., Miroshnichenko, A. E., Fu, Y. H., Zhang, J., \& Luk'yanchuk, B. (2012). Magnetic light. Scientific Reports, 2, 492.

4. Zel'dovich, I. B. (1958). Electromagnetic interaction with parity violation. Sov. Phys. JETP, 6(6), 1184-1186.

5. Flambaum, V. V., \& Murray, D. W. (1997). Anapole moment and nucleon weak interactions. Physical Review C, 56(3), 1641.

6. Dubovik, V. M., \& Tugushev, V. V. (1990). Toroid moments in electrodynamics and solid-state physics. Physics Reports, 187(4), 145-202.

7. Afanasiev, G. N., \& Dubovik, V. M. (1998). Some remarkable charge-current configurations. Physics of Particles and Nuclei, 29(4), 366-391.

8. Leforestier, A., \& Livolant, F. (2009). Structure of toroidal DNA collapsed inside the phage capsid. Proceedings of the National Academy of Sciences, 106(23), 9157-9162.

9. Vretenar, D., Paar, N., Ring, P., \& Nikšić, T. (2002). Toroidal dipole resonances in the relativistic random phase approximation. Physical Review C, 65(2), 021301.

10. Armani, D. K., Kippenberg, T. J., Spillane, S. M., \& Vahala, K. J. (2003). Ultra-highQ toroid microcavity on a chip. Nature, 421(6926), 925-928.

11. Dubovik, V. M., Martsenyuk, M. A., \& Saha, B. (2000). Material equations for electromagnetism with toroidal polarizations. Physical Review E, 61(6), 7087.

12. Spaldin, N. A., Fiebig, M., \& Mostovoy, M. (2008). The toroidal moment in condensed-matter physics and its relation to the magnetoelectric effect. Journal of Physics: Condensed Matter, 20(43), 434203.

13. Naumov, I. I., Bellaiche, L., \& Fu, H. (2004). Unusual phase transitions in ferroelectric nanodisks and nanorods. Nature, 432(7018), 737-740.

14. Savinov, V., Fedotov, V. A., \& Zheludev, N. I. (2014). Toroidal dipolar excitation and macroscopic electromagnetic properties of metamaterials. Physical Review B, 89(20), 205112.

15. Ceulemans, A., Chibotaru, L. F., \& Fowler, P. W. (1998). Molecular anapole moments. Physical Review Letters, 80(9), 1861. 
16. Flambaum, V. V., Khriplovich, I. B., \& Sushkov, O. P. (1984). Nuclear anapole moments. Physics Letters B, 146(6), 367-369.

17. Schmid, H. (2001). On ferrotoroidics and electrotoroidic, magnetotoroidic and piezotoroidic effects. Ferroelectrics, 252(1), 41-50.

18. Kaelberer, T., Fedotov, V. A., Papasimakis, N., Tsai, D. P., \& Zheludev, N. I. (2010). Toroidal dipolar response in a metamaterial. Science, 330(6010), 1510-1512.

19. Ding, C., Jiang, L., Sun, C., Wu, L., Xu, D., Zhang, G., \& Yao, J. (2015). Stable terahertz toroidal dipolar resonance in a planar metamaterial. Physica Status Solidi (b), 252(6), 1388-1393.

20. Huang, Y. W., Chen, W. T., Wu, P. C., Fedotov, V., Savinov, V., Ho, Y. Z., Chau, Y. F., Zheludev, N. I. and Tsai, D. P. (2012). Design of plasmonic toroidal metamaterials at optical frequencies. Optics Express, 20(2), 1760-1768.

21. Li, J., Zhang, Y., Jin, R., Wang, Q., Chen, Q., \& Dong, Z. (2014). Excitation of plasmon toroidal mode at optical frequencies by angle-resolved reflection. Optics Letters, 39(23), 6683-6686.

22. Jackson, J. D. (1999). Classical electrodynamics. (Vol. 3). Wiley, New York.

23. Radescu, E. E., \& Vaman, G. (2002). Exact calculation of the angular momentum loss, recoil force, and radiation intensity for an arbitrary source in terms of electric, magnetic, and toroid multipoles. Physical Review E, 65(4), 046609.

24. Costescu, A., \& Radescu, E. E. (1987). Induced toroid structures and toroid polarizabilities. Physical Review D, 35(11), 3496.

25. Miroshnichenko, A. E., Evlyukhin, A. B., Yu, Y. F., Bakker, R. M., Chipouline, A., Kuznetsov, A. I., Luk'yanchuk, B., Chichkov, B. N. \& Kivshar, Y. S. (2015). Nonradiating anapole modes in dielectric nanoparticles. Nature Communications, 6, 8069.

26. Arfken, G. B., and Weber, H. J. (2001). Mathematical Methods for Physicists (Vol. 5). (Harcourt/Academic, Oxford, 2001).

27. Huang, Y. W., Chen, W. T., Wu, P. C., Fedotov, V., Savinov, V., Ho, Y. Z., Chau, Y. F., Zheludev, N. I. \& Tsai, D. P. (2012). Design of plasmonic toroidal metamaterials at optical frequencies. Optics Express, 20(2), 1760-1768. 
28. Soukoulis, C. M., \& Wegener, M. (2011). Past achievements and future challenges in the development of three-dimensional photonic metamaterials. Nature Photonics, 5(9), 523-530.

29. Kern, A. M., \& Martin, O. J. (2009). Surface integral formulation for 3D simulations of plasmonic and high permittivity nanostructures. J. Opt. Soc. Am. A, 26(4), 732740.

30. Fedotov, V. A., Rogacheva, A. V., Savinov, V., Tsai, D. P., \& Zheludev, N. I. (2013). Resonant transparency and non-trivial non-radiating excitations in toroidal metamaterials. Scientific Reports, 3, 2967.

31. Ahmadivand, A., Gerislioglu, B., \& Pala, N. (2017). Large-Modulation-Depth Polarization-Sensitive Plasmonic Toroidal Terahertz Metamaterial. IEEE Photonics Technology Letters, 29(21), 1860-1863.

32. Ahmadivand, A., \& Golmohammadi, S. (2013). Electro-optic wavelength filtering device for plasmon waveguides based on ordered arrays of Au nanorings. OptikInternational Journal for Light and Electron Optics, 124(17), 2743-2745.

33. Ahmadivand, A., Golmohammadi, S., Karabiyik, M., \& Pala, N. (2015). Selfassembled silicon-based clusters to design efficient, fast, and controllable Fano switches. Microwave and Optical Technology Letters, 57(5), 1242-1246.

34. Nooshnab, V., \& Ahmadivand, A. (2017). Optothermally Functional Charge Transfer Plasmon Modulator. IEEE Photonics Technology Letters, 29(18), 1556-1559.

35. Ahmadivand, A., Gerislioglu, B., \& Pala, N. (2017). Active control over the interplay between the dark and hidden sides of plasmonics using metallodielectric $\mathrm{Au}-\mathrm{Ge}_{2} \mathrm{Sb}_{2} \mathrm{Te}_{5}$ unit cells. J. Phys. Chem. C, 121(36), 19966-19974.

36. Ahmadivand, A., Karabiyik, M., Sinha, R., Gerislioglu, B., \& Pala, N. (2016). Tunable terahertz response of plasmonic Vee-shaped assemblies with a graphene monolayer. IEEE Progress in Electromagnetic Research Symposium (PIERS), 2387.

37. Wang, Q., Rogers, E. T., Gholipour, B., Wang, C. M., Yuan, G., Teng, J., \& Zheludev, N. I. (2016). Optically reconfigurable metasurfaces and photonic devices based on phase change materials. Nature Photonics, 10(1), 60-65. 
38. Li, P., Yang, X., Maß, T. W., Hanss, J., Lewin, M., Michel, A. K. U., Wuttig, M. \& Taubner, T. (2016). Reversible optical switching of highly confined phononpolaritons with an ultrathin phase-change material. Nature Materials, 15(8), 870-875.

39. Michel, A. K. U., Chigrin, D. N., Maß, T. W., Schönauer, K., Salinga, M., Wuttig, M., \& Taubner, T. (2013). Using low-loss phase-change materials for mid-infrared antenna resonance tuning. Nano Letters, 3(8), 3470-3475.

40. Steele, J. M., Grady, N. K., Nordlander, P., \& Halas, N. J. (2007). Plasmon Hybridization in Complex Nanostructures (Vol. 1). Springer Netherlands.

41. Ahmadivand, A., \& Pala, N. (2015). Plasmon resonance hybridization in selfassembled copper nanoparticle clusters: Efficient and precise localization of surface plasmon resonance (LSPR) sensing based on Fano resonances. Applied Spectroscopy, 69(2), 277-286.

42. Ahmadivand, A., \& Golmohammadi, S. (2015). Fano resonances in complex plasmonic super-nanoclusters: The effect of environmental modifications on the LSPR sensitivity. Frontiers of Physics, 10(2), 222-230.

43. Miroshnichenko, A. E., Flach, S., \& Kivshar, Y. S. (2010). Fano resonances in nanoscale structures. Reviews of Modern Physics, 82(3), 2257.

44. Lee, K. L., Wu, S. H., Lee, C. W., \& Wei, P. K. (2011). Sensitive biosensors using Fano resonance in single gold nanoslit with periodic grooves. Optics Express, 19(24), 24530-24539.

45. Vasinajindakaw, P., Vaillancourt, J., Gu, G., Liu, R., Ling, Y., \& Lu, X. (2011). A Fano-type interference enhanced quantum dot infrared photodetector. Applied Physics Letters, 98(21), 211111.

46. Basharin, A. A., Kafesaki, M., Economou, E. N., Soukoulis, C. M., Fedotov, V. A., Savinov, V., \& Zheludev, N. I. (2015). Dielectric metamaterials with toroidal dipolar response. Physical Review X, 5(1), 011036.

47. Spaldin, N. A., Fiebig, M., \& Mostovoy, M. (2008). The toroidal moment in condensed-matter physics and its relation to the magnetoelectric effect. Journal of Physics: Condensed Matter, 20(43), 434203. 
48. Dubovik, V. M., \& Tugushev, V. V. (1990). Toroid moments in electrodynamics and solid-state physics. Physics Reports, 187(4), 145-202. 


\section{CHAPTER 6}

\section{Plasmonics for Photothermal Heat Generation}

Plasmonics has provided exquisite features and opened new avenues for developing promising advanced medical techniques including but not limited to biosensing $[1,2]$, fluorescence spectroscopy [3,4], tumor and cancer therapies [5-7], photothermal heat generation [8-11], cell and optoacoustic imaging [12,13], drug delivery and nanomedicine [14-16], nanosurgery [17], neuron stimulation [18,19], DNA assays [20]. Such broad and extensive applications of plasmonics in modern nanomedical sciences have stimulated researchers to exploit the associated exotic features for developing novel medical and clinical devices and apertures [21]. In the following sections, we briefly summarize our studies and achievements in nano- and microscale plasmonics for photothermal heat generation.

\subsection{Photothermal Heat Generation}

Photothermal heat generation in metallic subwavelength structures in a short time scale has been extensively utilized in biological applications [22], photothermal cancer and tumor therapies [23-25], bubble formation [26], control of enzyme reaction [27], etc. All these applications show the importance of photothermal heat flux production in subwavelength plasmonic structures for advanced clinical applications. For the noble metallic particles, LSPRs in nanosize structures with absorptive behavior leads to tremendous dissipation of an incident optical energy [28]. This large amount of light absorption can be achieved in an extremely short time interval by the free electron gas through electron-electron scattering in a picosecond time scale at the metal-dielectric boundary, and results in photothermal heat generation [29,30]. The photothermal 
responses of various shapes of nanoparticles in different orientations have been investigated analytically and experimentally, and also, the effects of structural, optical, and environmental parameters were included in these analysis [8,31]. Considering experimentally measured refractive index and dielectric constant [32], thermal conductivity [33], and specific thermal heat capacity [34] for several substances, Ag and Al have emerged as the metals of choice for light-to-heat conversion. In the past decade, hybridization of plasmon resonances was introduced as one of the most important mechanisms in excitation and intensification of plasmon resonant modes inside and between metallic molecular nanoparticles [35]. This phenomenon can be understood by analyzing the plasmon response of simple nanoparticle assemblies such as dimers and trimers. The formation of Fano lineshape and the unique behavior of this resonant mode were analyzed in Chapter 3, comprehensively. Technically, at this point a high amount of optical energy accumulates in the structure and can be controlled based on application. Behaviors of hybridized and localized plasmon resonant modes and also Fano mode depend on the material and geometrical properties of nanoparticles. A simple evaluation between noble metals showed that Ag provides a remarkable absorption of optical energy in the visible to the NIR due to Ohmic losses and also the plasmon resonances excitation here depending on the complexity of the aggregate [36,37]. On the other hand, very recently, cascaded plasmon resonant modes in simple compositional trimer clusters have been used in light to heat conversion process which resulted a significant temperature change with picoseconds relaxation time [38]. Using inanoparticles with high geometrical tunability and absorptive properties yield remarkable enhancement in photothermal response. Comparing the geometrical properties of various nanoparticles, core-shell 
particles and cavities provide unique geometrical tunability which can be employed to design highly symmetrical nanoparticle assemblies to support strong resonances $[39,40]$. In the recent years, there have been great efforts to increase the temperature and efficiency of photothermal heat flux production in nanoplasmonic devices [8,11]. However, the highest possible temperature variations that have been obtained was around $\sim 100 \mathrm{~K}$ with the power flux of near to $\sim 50 \mu \mathrm{Wcm}^{-2}$. Here, we study the plasmon and photothermal responses for a quadrumer cluster comprises of $\mathrm{Ag}$ core-shell nanostructures in free space and aqueous ambiences. Using plasmon hybridization theory, we analyzed and compared the plasmon response for metallic and metallodielectric quadrumers. It is proved that a symmetric and identical four-member cluster is able to support pronounced Fano dip during laser pump exposure. Determining the photothermal heat energy flux produced by the cluster, we quantified the temperature changes numerically and theoretically. Then, with the placement of carbon nanospheres in the offset gap space between nanoparticles, we introduced a metallodielectric molecular assembly to enhance the photothermal efficiency. Analyzing the photothermal response of the proposed metallodielectric nanostructure, we enhanced the quality of Fano minimum by inducing new magnetic dark modes by tiny carbon nanospheres. It is verified that this structural modification can result in a dramatic enhancement in the temperature. Figures 6.1a-6.1c exhibit schematic diagrams for the proposed quadrumer cluster with the description of geometrical parameters inside and also in both metallic and metallodielectric regimes. Four identical Ag core-shell nanoparticles were located with the dimensions of $r_{d} / r_{b} / r_{d} / h=60 / 105 / 140 / 85 \mathrm{~nm}$ close to each other with an offset distance of $D_{4 \mathrm{q}}=15 \mathrm{~nm}$ (see the inset in Fig. 6.1d). Then we calculated the scattering and 

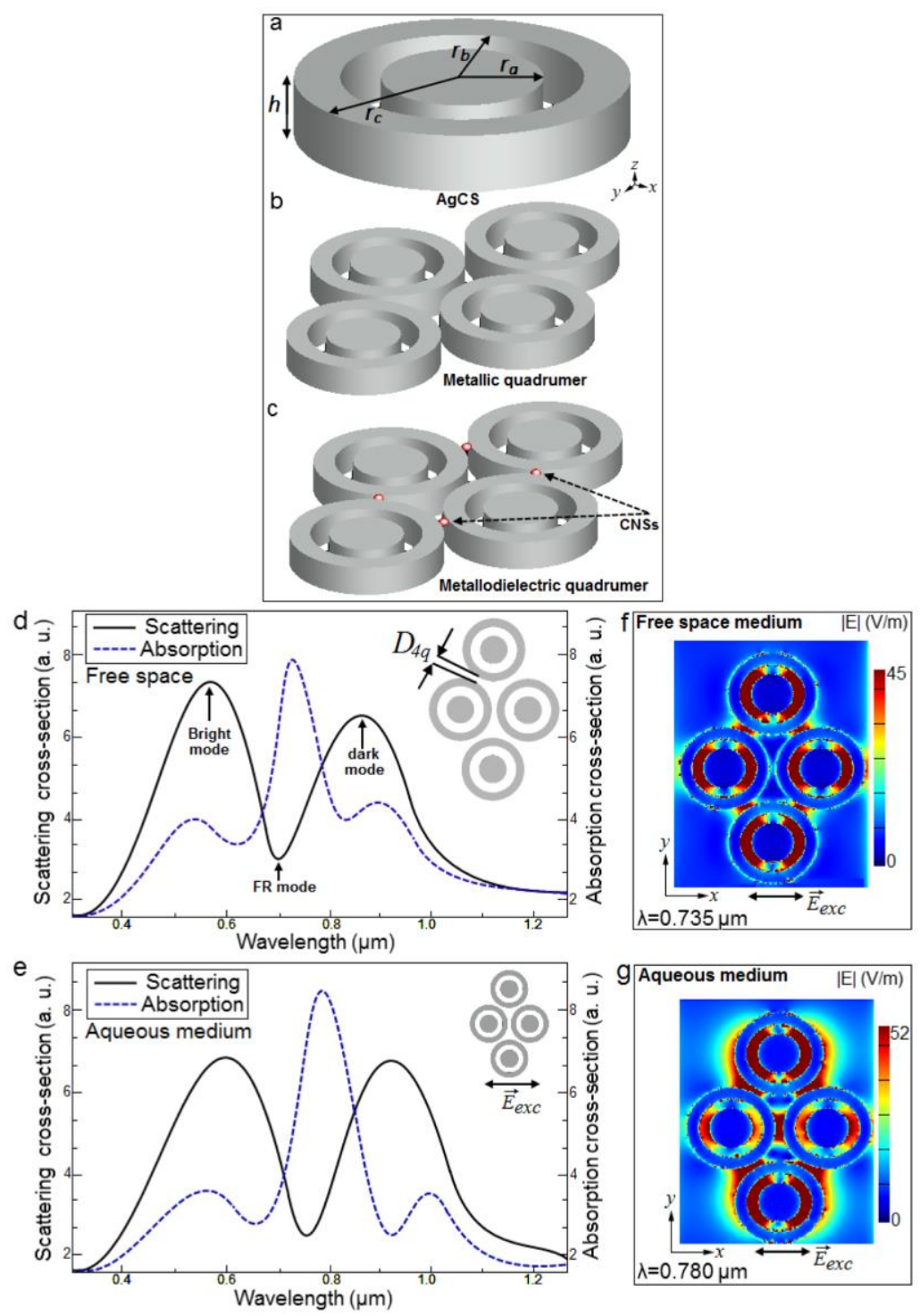

Figure 6.1. a, b, c) Schematic diagrams for an Ag nanoparticle and a four-member quadrumer composed of nanoparticles in both metallic and metallodielectric regimes, respectively. d, e) Scattering and absorption profiles for the metallic quadrumer in the free space and aqueous ambiences under transverse polarization excitation $\left(E_{\text {exc }}\right)$. The insets show the gap distance between core-shell units and E-field illumination direction. f, g) E-field profiles during plasmon resonance excitations coupling in the metallic quadrumer in free space and liquid systems.

absorption profiles for the quadrumer cluster in free space and water which are shown Figs. 6.1d and 6.1e, respectively. For free space $(n=1)$, a noticeable Fano mode is induced at the visible spectrum $(\lambda=0.690 \mu \mathrm{m})$, while for the fluid system $(n=1.33)$ like biological mechanisms, the Fano lineshape is red-shifted to the longer spectra $(\lambda=0.760 \mu \mathrm{m})$ and 
also becomes deeper due to high amount of energy accumulation at this position [41]. This red-shift in the position of Fano dip accompanies with a noticeable field intensification at the resonance frequency $\left(\omega_{\text {LSPR }}\right)$. In addition, a huge amount of power dissipation is recorded at the resonance wavelength. Noticing in the numerically obtained E-field maps as two-dimensional snapshots for each profile (see Figs. 6.1f and 6.1g), the plasmon resonance enhancement in the aqueous ambience is superior and the excitation of plasmon resonances in all of the nanoparticle members is achieved. Also, in this system, the EM energy is localized in the offset gaps between nanoparticles and in the space between the core and shell parts. This strong localization of plasmon modes can lead to large amount of heat energy production. In this regime, we expect an enhanced photothermal power generation at sub-nanosecond time scale according to the incident Gaussian pulse length ( $2.65 \mathrm{fs})$. The amount of power dissipation and heat generation can be analyzed in nanoscale dimensions considering all the parameters for the structural and environmental characteristics using the following equation [42]:

$$
\Delta T=\frac{C_{a b s} \varphi}{c V \rho}
$$

Where $C_{a b s}$ is the, absorption coefficient $\varphi$ is the optical flux of the incident light source $\left(\mathrm{Jm}^{-2}\right), c$ is the specific heat capacity of the core-shell nanoparticles $\left(\mathrm{JKg}^{-1} \mathrm{~K}^{-1}\right)$ NPs, $\Delta T$ is the temperature variations in a specific time scale $(\mathrm{K}), \rho$ is the density of the particles $\left(\mathrm{Kgm}^{-3}\right) \mathrm{NPs}$, and $V$ is the volume of the entire nanocluster $\left(\mathrm{m}^{3}\right)$. Practically, the mechanism of photothermal heat generation and transfer in nanoscale is a nonequilibrium process. Hence, we assume that the phonons interact with the cluster as a hot object, with the aqueous medium as a cool object and travel in this ambience. Considering the amount 


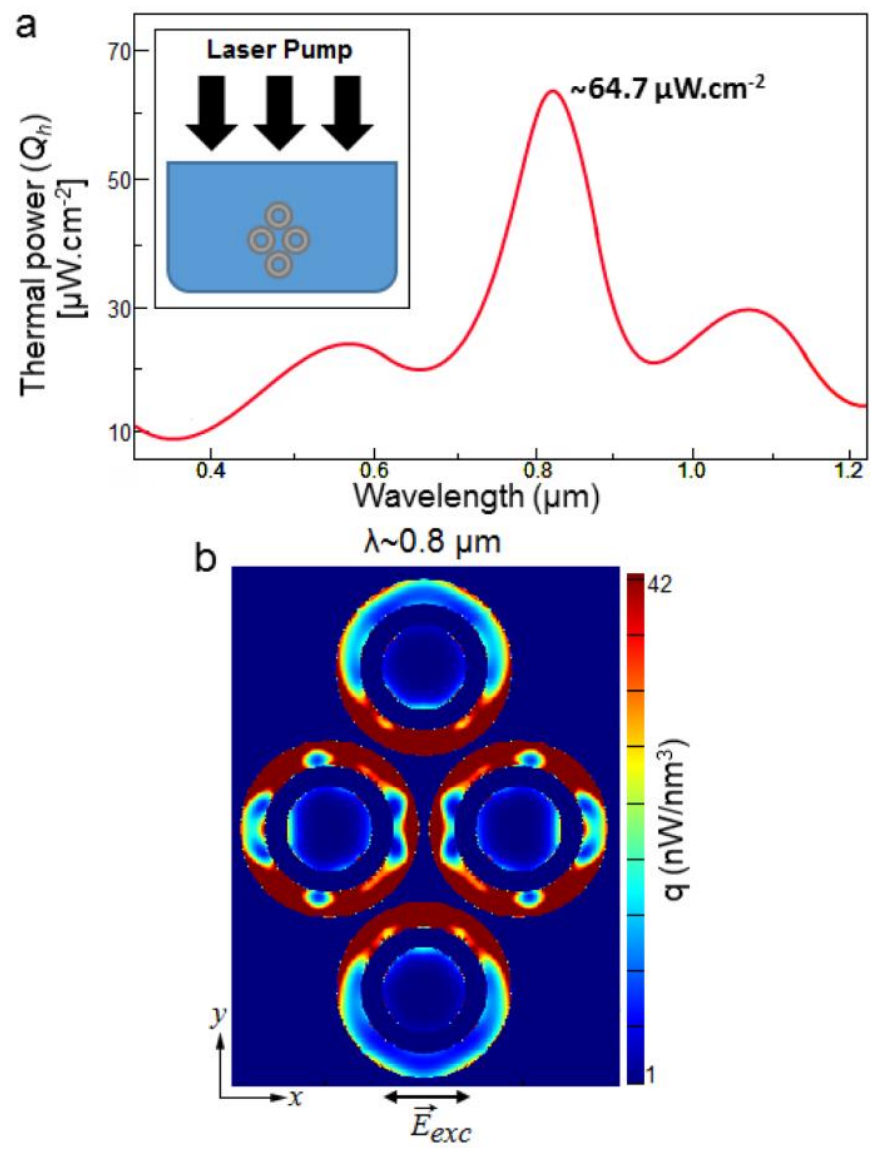

Figure 6.2. a) Thermal heat power flux $\left(Q_{h}\right)$ profile for a metallic nanoassembly during laser pump exposure in a liquid system. b) Dissipated power density mapping in a metallic quadrumer at the peak of absorption.

of the absorbed power and environmental properties, the absorption coefficient at $\omega_{L S P R}$ is given by:

$$
\left.C_{a b s}\right|_{\omega_{L S P R}}=\frac{2 \int P_{L S P R} d V}{n_{m} \sqrt{\varepsilon_{0} / \mu_{0}\left|E_{e x c}\right|^{2}}}
$$

where $P_{\text {LSPR }}$ is the optical power loss density per volume that is absorbed by nanoparticles based on Ohmic losses of core-shell units at $\omega_{\text {LSPR }}, n_{m}$ is the refractive index of the surrounding medium, $\varepsilon_{0}$ and $\mu_{0}$ are the permittivity and permeability of the free space, respectively, and $E_{\text {exc }}$ is the amplitude of the incident transverse electric field. 
Here, we put into the account, the amount of energy that was absorbed at $\omega_{\text {LSPR }}$, and then, we determined the photothermal heat generated in a very short time scale by setting the relevant simulation parameters in picoseconds. Figure 6.2a shows the total generated heat flux $\left(Q_{\mathrm{h}}\right)$ by the quadrumer cluster in the water medium, which can be quantified at $\omega_{\text {LSPR }}$ by using the equation: $Q_{h}=0.5\left(n_{m} c_{0} \varepsilon_{0}\left|\mathrm{E}_{0}\right| 2 C_{a b s}\right)$ proposed by Baffou et al. [8]. More than the distinct extreme that is appeared for the position of Fano minimum, we also observed
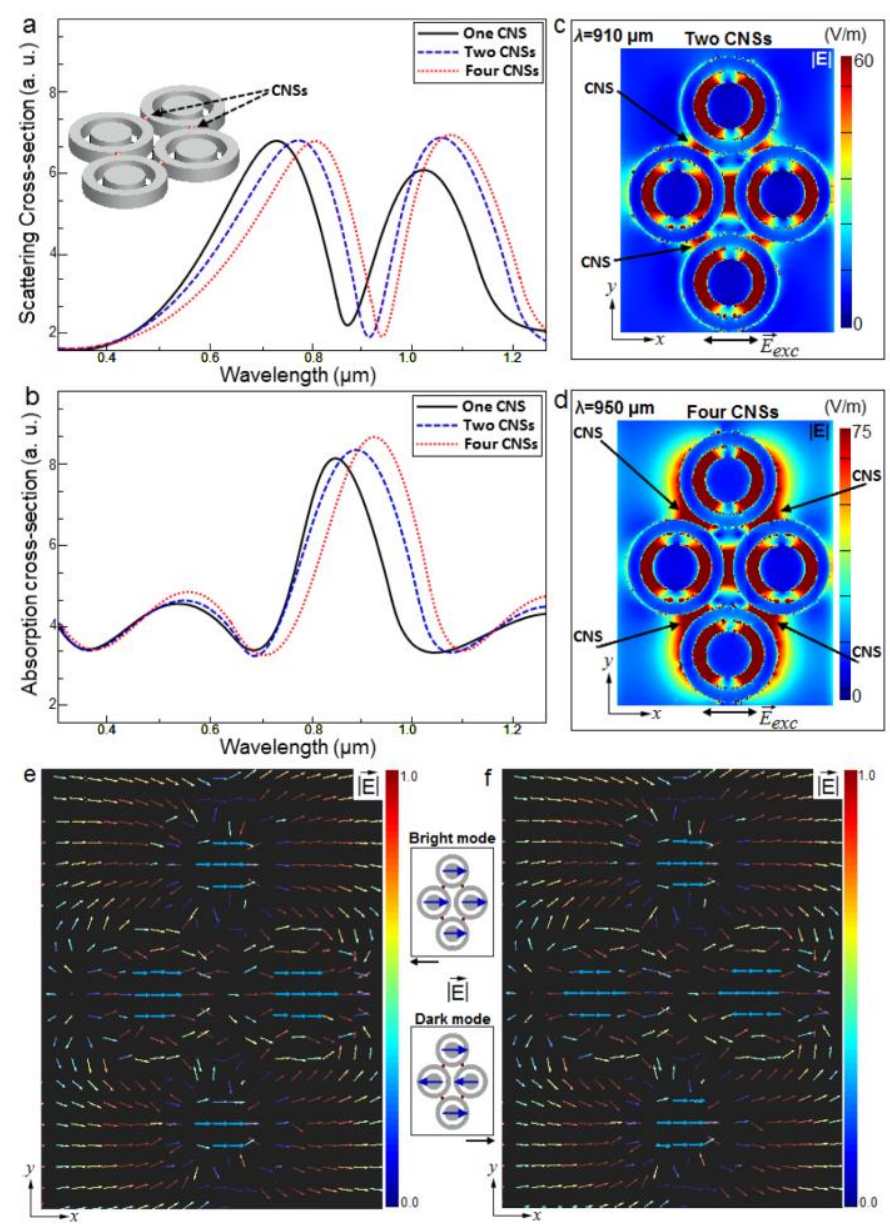

Figure 6.3. a, b) Scattering and absorption spectra for quadrumer cluster with the carbon nanospheres with different quantities in an aqueous system. c, d) The electric field profile $|\mathrm{E}|$ showing the hybridization and enhancement in metallodielectric quadrumer clusters with two and four CNSs, respectively. e, f) The electric field distribution diagram inside the metallodielectric quadrumer with four carbon nanospheres at Fano dip position for bright and dark modes. Carbon nanospheres are denoted by CNS in the picture. 
a couple of shoulders at the visible $(\sim 0.580 \mu \mathrm{m})$ and NIR $(\sim 1.1 \mu \mathrm{m})$ spectra which are correlated with small amount of optical power absorption at the bonding and antibonding resonant modes positions, respectively. For the considered setting above, we calculated the temperature changes as $\Delta T=150 \mathrm{~K}$ for a metallic quadrumer cluster composed of $\mathrm{Ag}$ nanoparticle units with the optical fluence of the incident pulse of $20 \mathrm{Jm}^{-2}$. The energy dissipation inside the metallic quadrumer cluster at $\omega_{\text {LSPR }}$ is shown in Fig. $6.2 \mathrm{~b}$ as heat power map. It should be underlined that such a high temperature is obtained in a subnanoscale time duration due to high dissipation of optical power inside the quadrumer cluster.

However, this performance of the proposed nanocluster can be further enhanced by utilizing plasmon transmutation effect [43]. To this end, we modified the plasmon response of the quadrumer cluster with the placement of carbon nanospheres to the offset junctions between proximal nanoparticles (Ag-C-Ag). For the carbon spheres, we employed carbon particles with the experimentally determined complex permittivity as $\varepsilon=2.25+i 0.0215$, and absorption coefficient, $\alpha=1531.5 \mathrm{~cm}^{-1}$ [44]. The plasmon transmuting in a four-member subwavelength cluster includes inducing collective antibonding magnetic plasmon resonant modes which leads to intensifying the energy of the induced Fano dip. To show the effect of carbon nanospheres placement to the metallic quadrumer cluster, we calculated the photothermal response for the metallodielectric nanocomplex with breaking the symmetry of the cluster due to the presence of the nanospheres. It should be noted that due to the concentration of hybridized plasmon resonant modes at the edge-to-edge distances (gaps) between core-shell units, we expect more heat generation in these regions. The placement of the carbon nanospheres in the 
gaps accompanied by the formation of strong collective magnetic modes results in large plasmon resonance energy accumulation at these gaps. Figures $6.3 \mathrm{a}$ and $6.3 \mathrm{~b}$ exhibit the scattering and absorption cross-sectional profiles for the metallodielectric assembly, respectively. It is observed that placement of two carbon spheres with the radii of $14 \mathrm{~nm}$ in the junction between $\mathrm{Ag}-\mathrm{C}$ nanoparticles makes corresponding Fano dip deeper, meanwhile its position red-shifts to the longer wavelengths due to symmetry cancellation caused by addition of carbon particles. Considering the plasmon resonance hybridization mechanism, with the placement of one or two carbon spheres at the left side of the quadrumer, collective magnetic subradiant modes could be induced, while a symmetry breaking can be performed as a result of this metal-dielectric contribution. Formation of collective magnetic dark modes can result in a deeper Fano minimum. However, comparing the effect of symmetry breaking and the carbon spheres deposition, the role of dark modes induced by carbon spheres in Fano minimum intensification is significant. Hence, increasing the number of carbon nanospheres at the offset gaps between Ag-C nanoparticles leads to formation of enhanced subradiant modes. Therefore, for the quadrumer cluster with four carbon spheres, a sharper dip appears at $\lambda=0.950 \mu \mathrm{m}$, corresponding to the strong hotspots. Note that in the depicted scattering spectra in Fig. 6.3a, increasing the number of the deposited carbon nanospheres at the gaps, red-shifts the Fano spectral feature and leads to narrower and deeper dips. The inset in Fig. 6.3a shows a schematic for the examined quadrumer with the placement of carbon spheres, while the illumination and polarization directions are indicated by arrows. Figure $6.3 \mathrm{c}$ displays the field distribution and E-field map for the plasmon resonance hybridization and excitation inside a metallodielectric quadrumer with two carbon nanoparticles in the 
left side of the quadrumer (the location of dielectric spheres are indicated by the arrows).

For the metallodielectric quadrumer with four carbon particles, analyzing the photothermal response of the latest nanocomplex immersed in the aqueous ambience (see E-map profile depicted in Fig. 6.3d), we found a significant improvement in the electric field enhancement in comparison to the fully metallic quadrumer and a cluster with two carbon nanoparticles. In order to show the behavior of quadrumer at the position of bright and dark modes, we plotted electric field distribution diagram for both of the modes, as shown in Figs. 6.3e and 6.3f, respectively. Noticing in the depicted arrows, in Fig. 6.3e all of the dipolar plasmons oscillate in-phase and in the same direction in all of the metallodielectric units. On the other hand, considering Fig. 6.3d, the dipolar moments of two units are in the opposite direction of the other units, which is in complete agreement
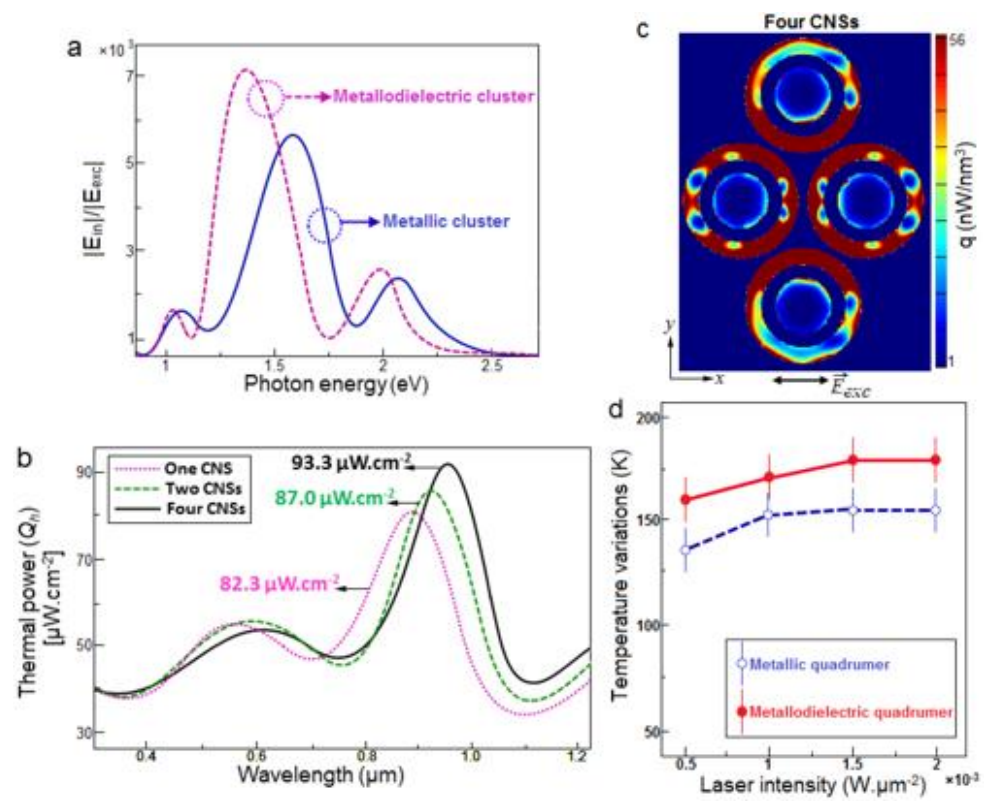

Figure 6.4. a) Field enhancement factor $\left(\left|E_{i n}\right| /\left|E_{\text {exc }}\right|\right)$ over the photon energy for both metallic and metallodielectric (with four carbon nanospheres) clusters in an aqueous ambience. b) Photothermal heat power flux $\left(Q_{h}\right)$ spectra at the interparticle junction for a metallodielectric assembly with carbon nanospheres. c) Photothermal heat density in quadrumer with four carbon spheres. d) Photothermal heat temperature variations over the laser pulse intensity for both metallic and metallodielectric plasmonic clusters. Carbon nanospheres are denoted by CNS in the picture. 
with the plasmon hybridization theory. Figure 6.4a shows the internal electric field enhancement factor $\left(\left|E_{\text {in }}\right|\left|E_{\text {exc }}\right|\right)$ which is the ratio of the overall localized field in the nanoparticles gaps and in the space between cores and shells to the incoming electric field for the clusters in all the examined structural alterations. This field enhancement is calculated at the Fano dip position. Comparing this parameter for both metallic and metallodielectric (with four carbon nanoparticles) clusters, a significant enhancement in the electric field intensity for the metallodielectric structure with four carbon nanoparticles is observed. Figure $6.4 \mathrm{~b}$ shows numerically computed photothermal heat flux where the peak of power is red-shifted to the longer spectra by increasing the number of carbon nanospheres. For the cluster with four carbon spheres, the photothermal heat power flux is calculated as $93.3 \mu \mathrm{W} . \mathrm{cm}^{-2}$. Figure $6.4 \mathrm{c}$ exhibits the absorbed power loss density by nanoparticles as a two-dimensional snapshot. Employing the method described above, temperature variation in the metallodielectric nanocomplex is estimated as $\Delta T=172 \mathrm{~K}$.

Finally, we examined the effect of variations in the intensity of the incident laser power on the photothermal heal temperature production as shown in Fig. $6.4 \mathrm{~d}$ for both metallic and metallodielectric quadrumer clusters. Using the proposed method by Fang et al. [45] that was proposed for plasmonic particles immersed in water medium, the temperature variations in the proposed nanoscale structure can be plotted as a function of incident laser source power. According to depicted $I-T$ curve, increasing the intensity of light up to $1.5 \mathrm{~mW} . \mu \mathrm{m}^{-2}$ leads to minor enhancements in the produced photothermal heat temperature, while for higher intensities $\left(I>1.5 \mathrm{~mW} . \mu \mathrm{m}^{-2}\right)$ temperature variation is very small and negligible, due to limited geometrical capacity of the quadrumer nanostructure 
in supporting high energies. For instance, for $I=2 \mathrm{~mW} \cdot \mu \mathrm{m}^{-2}$, change in the temperature due to generated thermal heat could not reach above $\Delta T \sim 180 \mathrm{~K}$ (saturating condition). Also, it should be underlined that for practical applications, such a high power can result in destructive effects such as defects in the geometries of the cluster. Carbon nanotubes and graphene sheets [16] and more recently plasmonic nanoshells and nanomatryoshkas [46] have been employed in bio-medical applications such as tumor and cancer therapies. Here, we proposed a numerical method to analyze the behavior of a nanoplasmonic structure with the contribution of carbon nanospheres. Comparing the performance of the proposed nanostructure with the analogous ones that have been reported in the literature $[16,46]$, the plasmon response of the proposed structure is superior. Therefore, with their improved characteristics, we expect that the proposed metallodielectric nanostructures will make a great contribution to various bio-medical and photothermal spectroscopy applications.

\subsection{References}

1. Anker, J. N., Hall, W. P., Lyandres, O., Shah, N. C., Zhao, J., \& Van Duyne, R. P. (2008). Biosensing with plasmonic nanosensors. Nature Materials, 7(6), 442-453

2. Kabashin, A. V., Evans, P., Pastkovsky, S., Hendren, W., Wurtz, G. A., Atkinson, R., Pollard, R., Podolskiy, V. A., \& Zayats, A. V., 2009. Plasmonic nanorod metamaterials for biosensing. Nature Materials, 8(11), 867-871.

3. Liebermann, T., \& Knoll, W. (2000). Surface-plasmon field-enhanced fluorescence spectroscopy. Colloids and Surfaces A: Physicochemical and Engineering Aspects, 171(1), 115-130.

4. Olivio, M., \& Dinish, U. S. (2015) Frontiers in biophotonics for translational medicine (Vol. 1). Springer, Singapore.

5. Huang, X., Jain, P. K., El-Sayed, I. H., \& El-Sayed, M. A. (2008). Plasmonic photothermal therapy (PPTT) using gold nanoparticles. Lasers in Medical Science, 23(3), 217. 
6. Bardhan, R., Lal, S., Joshi, A., \& Halas, N. J. (2011). Theranostic nanoshells: from probe design to imaging and treatment of cancer. Accounts of Chemical Research, 44(10), 936.

7. Huang, X., Qian, W., El-Sayed, I. H., \& El-Sayed, M. A. (2007). The potential use of the enhanced nonlinear properties of gold nanospheres in photothermal cancer therapy. Lasers in surgery and medicine, 39(9), 747-753.

8. Baffou, G., Quidant, R., \& Girard, C. (2009). Heat generation in plasmonic nanostructures: Influence of morphology. Applied Physics Letters, 94(15), 153109.

9. Hao, J., Zhou, L., \& Qiu, M. (2011). Nearly total absorption of light and heat generation by plasmonic metamaterials. Physical Review B, 83(16), 165107.

10. Ahmadivand, A., Pala, N., \& Güney, D. Ö. (2015). Enhancement of photothermal heat generation by metallodielectric nanoplasmonic clusters. Optics Express, 23(11), A682-A691.

11. Toroghi, S., \& Kik, P. G. (2014). Photothermal response enhancement in heterogeneous plasmon-resonant nanoparticle trimers. Physical Review B, 90(20), 205414.

12. Huang, X., El-Sayed, I. H., Qian, W., \& El-Sayed, M. A. (2006). Cancer cell imaging and photothermal therapy in the near-infrared region by using gold nanorods. Journal of the American Chemical Society, 128(6), 2115-2120.

13. Mallidi, S., Larson, T., Aaron, J., Sokolov, K., \& Emelianov, S. (2007). Molecular specific optoacoustic imaging with plasmonic nanoparticles. Optics Express, 15(11), 6583-6588.

14. Sershen, S. R., Westcott, S. L., Halas, N. J., \& West, J. L. (2000). Temperaturesensitive polymer-nanoshell composites for photothermally modulated drug delivery. Journal of Biomedical Materials Research Part A, 51(3), 293-298.

15. Letfullin, R. R., \& George, T. F. (2013). Plasmonic nanomaterials for nanomedicine in Springer Handbook of Nanomaterials, Springer Berlin Heidelberg.

16. Chen, W., Ayala-Orozco, C., Biswal, N. C., Perez-Torres, C., Bartels, M., Bardhan, R., Stinnet, G., Liu, X. D., Ji, B., Deorukhkar, A. \& Brown, L. V. (2014). Targeting pancreatic cancer with magneto-fluorescent theranostic gold nanoshells. Nanomedicine, 9(8), 1209-1222.

17. Boulais, E., Lachaine, R., Hatef, A., \& Meunier, M. (2013). Plasmonics for pulsedlaser cell nanosurgery: Fundamentals and applications. Journal of Photochemistry and Photobiology C: Photochemistry Reviews, 17, 26-49. 
18. Zhang, J., Atay, T., \& Nurmikko, A. V. (2009). Optical detection of brain cell activity using plasmonic gold nanoparticles. Nano Letters, 9(2), 519-524.

19. Nakatsuji, H., Numata, T., Morone, N., Kaneko, S., Mori, Y., Imahori, H., \& Murakami, T. (2015). Thermosensitive Ion Channel Activation in Single Neuronal Cells by Using Surface-Engineered Plasmonic Nanoparticles. Angewandte Chemie International Edition, 54(40), 11725-11729.

20. Li, G., Zhu, L., He, Y., Tan, H., \& Sun, S. (2017). Digital triplex DNA assay based on plasmonic nanocrystals. Analytical and bioanalytical chemistry, 409(14), 36573666.

21. Li, J., Liu, J., \& Chen, C. (2017). Remote Control and Modulation of Cellular Events by Plasmonic Gold Nanoparticles: Implications and Opportunities for Biomedical Applications. ACS Nano, 11(3), 2403-2409.

22. Sperling, R. A., Gil, P. R., Zhang, F., Zanella, M., \& Parak, W. J. (2008). Biological applications of gold nanoparticles. Chemical Society Reviews, 37(9), 1896-1908.

23. Huang, X., El-Sayed, I. H., Qian, W., \& El-Sayed, M. A. (2006). Cancer cell imaging and photothermal therapy in the near-infrared region by using gold nanorods. Journal of the American Chemical Society, 128(6), 2115-2120.

24. Lal, S., Clare, S. E., \& Halas, N. J. (2008). Nanoshell-enabled photothermal cancer therapy: impending clinical impact. Accounts of Chemical Research, 41(12), 18421851.

25. Guo, R., Zhang, L., Qian, H., Li, R., Jiang, X., \& Liu, B. (2010). Multifunctional nanocarriers for cell imaging, drug delivery, and near-IR photothermal therapy. Langmuir, 26(8), 5428-5434.

26. Neumann, O., Urban, A. S., Day, J., Lal, S., Nordlander, P., \& Halas, N. J. (2012). Solar vapor generation enabled by nanoparticles. ACS Nano, 7(1), 42-49.

27. Blankschien, M. D., Pretzer, L. A., Huschka, R., Halas, N. J., Gonzalez, R., \& Wong, M. S. (2012). Light-triggered biocatalysis using thermophilic enzyme-gold nanoparticle complexes. ACS Nano, 7(1), 654-663.

28. Sun, Z., \& Zuo, X. (2011). Tunable absorption of light via localized plasmon resonances on a metal surface with interspaced ultra-thin metal gratings. Plasmonics, 6(1), 83-89.

29. Maradudin, A. A., Sambles, J. R., \& Barnes, W. L. (2014). Modern Plasmonics (Vol. 4). Elsevier, Poland. 
30. Baffou, G., \& Quidant, R. (2013). Thermo-plasmonics: using metallic nanostructures as nano-sources of heat. Laser \& Photonics Reviews, 7(2), 171-187.

31. Govorov, A. O., \& Richardson, H. H. (2007). Generating heat with metal nanoparticles. Nano Today, 2(1), 30-38.

32. Kutz, M. (2002). Handbook of materials selection. John Wiley \& Sons.

33. Shindé, S. L., \& Goela, J. (2006). High thermal conductivity materials (Vol. 91). New York, Springer.

34. Parker, W. J., Jenkins, R. J., Butler, C. P., \& Abbott, G. L. (1961). Flash method of determining thermal diffusivity, heat capacity, and thermal conductivity. Journal of Applied Physics, 32(9), 1679-1684.

35. Wang, H., Brandl, D. W., Nordlander, P., \& Halas, N. J. (2007). Plasmonic nanostructures: artificial molecules. Accounts of Chemical Research, 40(1), 53-62.

36. Zohar, N., \& Haran, G. (2014). Modular plasmonic antennas built of ultrathin silicashell silver-core nanoparticles. Langmuir, 30(26), 7919-7927.

37. Ahmadivand, A., \& Pala, N. (2016). Analyzing Photothermal Heat Generation Efficiency in a Molecular Plasmonic Silver Nanomatryushka Dimer. Plasmonics, 11(2), 493-501.

38. Toroghi, S., \& Kik, P. G. (2014). Photothermal response enhancement in heterogeneous plasmon-resonant nanoparticle trimers. Physical Review B, 90(20), 205414 .

39. Ahmadivand, A., \& Pala, N. (2015). Tailoring the negative-refractive-index metamaterials composed of semiconductor-metal-semiconductor gold ring/disk cavity heptamers to support strong Fano resonances in the visible spectrum. J. Opt. Soc. Am. A, 32(2), 204-212.

40. Ahmadivand, A., Sinha, R., \& Pala, N. (2015). Graphene plasmonics: multiple sharp Fano resonances in silver split concentric nanoring/disk resonator dimers on a metasurface. SPIE Nanoscience + Engineering, 954713.

41. Hao, F., Sonnefraud, Y., Dorpe, P. V., Maier, S. A., Halas, N. J., \& Nordlander, P. (2008). Symmetry breaking in plasmonic nanocavities: subradiant LSPR sensing and a tunable Fano resonance. Nano Letters, 8(11), 3983-3988.

42. Jackson, J. B., \& Halas, N. J. (2001). Silver nanoshells: variations in morphologies and optical properties. The Journal of Physical Chemistry B, 105(14), 2743-2746. 
43. Shafiei, F., Monticone, F., Le, K. Q., Liu, X. X., Hartsfield, T., Alù, A., \& Li, X. (2013). A subwavelength plasmonic metamolecule exhibiting magnetic-based optical Fano resonance. Nature Nanotechnology, 8(2), 95-99.

44. Djurišić, A. B., \& Li, E. H. (1999). Optical properties of graphite. Journal of Applied Physics, 85(10), 7404-7410.

45. Fang, Z., Zhen, Y. R., Neumann, O., Polman, A., García de Abajo, F. J., Nordlander, P., \& Halas, N. J. (2013). Evolution of light-induced vapor generation at a liquidimmersed metallic nanoparticle. Nano Letters, 13(4), 1736-1742.

46. Moon, H. K., Lee, S. H., \& Choi, H. C. (2009). In vivo near-infrared mediated tumor destruction by photothermal effect of carbon nanotubes. ACS Nano, 3(11), 37073713. 


\section{CHAPTER 7}

\section{Terahertz Toroidal Plasmonic Metamaterial for Biomarker Protein Detection}

In previous chapters, the plasmonic response and the characteristics of toroidal metamolecules at the near-infrared to the $\mathrm{THz}$ spectra were discussed comprehensively. Unconventional characteristics of magnetic toroidal multipoles have triggered researchers to study these unique resonant phenomena by using both 3D and planar resonators under intense radiation. High-quality and ultra-narrow resonance lineshapes and substantial sensitivity of this mode to the environmental perturbations (i.e. refractive index of the medium) can be effectively used for identification of ultralow weight atomic proteins and bio-agents for biochemical and biological sensing purposes.

Utilizing the exotic features of toroidal moments in the THz band, in this chapter, we provide detailed reports on the using toroidal modes for advanced biosensing applications. To this end, we used our proposed and studies microstructure in Chapter 5. This chapter also demonstrates that how the high-quality factor toroidal dipolar moment can be excited in plasmonic multi-metallic unit cells. Then, by taking the advantage of high quality toroidal lineshape and its dependence on the environmental perturbations, in the current chapter, we demonstrate that room-temperature toroidal metasurface is a reliable platform for immunosensing applications. As a proof of concept, we utilized our plasmonic metasurface to detect Zika-virus (ZIKV) envelope proteins (with diameter of $40 \mathrm{~nm}$ and molecular weight of $\sim 13 \mathrm{kDa}$ ) using a specific ZIKV antibody. The sharp toroidal resonant modes of the surface functionalized structures shift as a function of the ZIKV envelope protein for small concentrations $(\sim \mathrm{pM})$. In the following sections, we first describe the characteristics and importance of the targeted infectious proteins in 

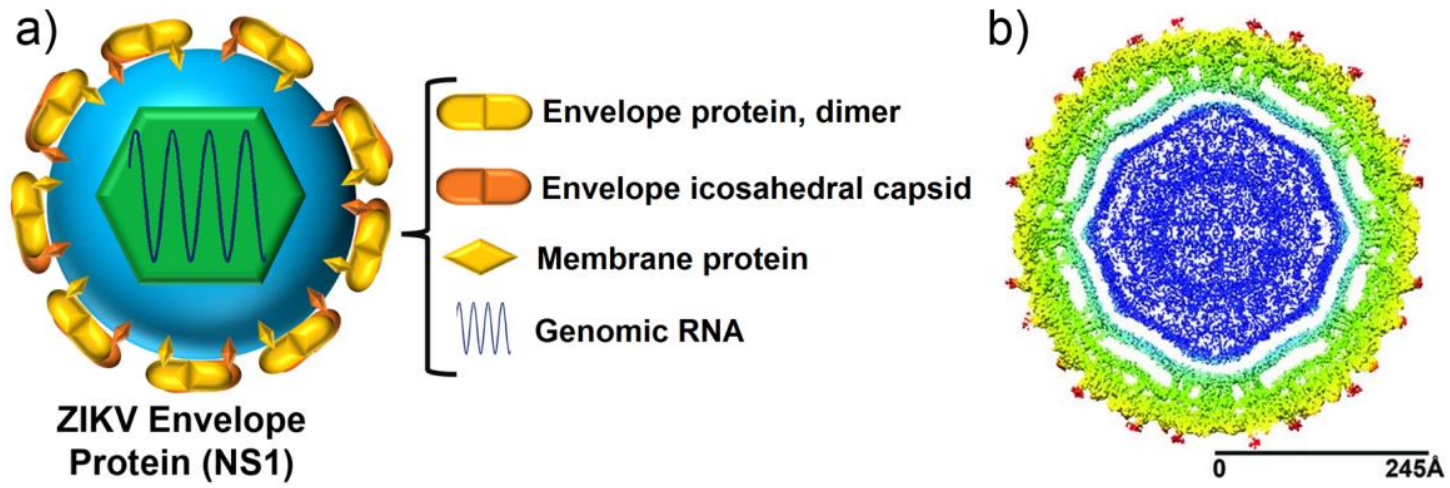

Figure 7.1 Contribution of the ZIKV external morphology and the cryo-electron microscopic image of hydrated ZIKV. a) An artistic representation of the ZIKV virion structure. b) The cross-section of cryo-electron microscopic illustration of ZIKV, showing the radial density distribution with specific color coding, obtained with permissions from Ref. [10].

details and the employed treatment and detection methods that have been proposed recently. Then, using the proposed toroidal metasurface, we qualitatively analyze the detection performance of ZIKV envelope proteins and the associated sensing parameters.

\subsection{Targeted Infectious (ZIKA-Virus)}

In this section, we briefly summarize the targeted infectious properties and practical trends in identification of disorder. Zika is a new medical threat across the world as an infectious disease which causes serious health disorders, possibly leading to death $[1,2]$. This type of infection spreads in humans through the mosquitoes Aedes aegypti and Aedes albopictus. The transmission mode consists of a mosquito biting an infected host, taking in infected blood containing the virus, and further injecting the infectious saliva into the healthy host system. This process ends in a serious infection, resulting in lifethreatening pathogenesis and disease progression [3]. ZIKV infection has various transmission modes [4] and mechanisms including from mother to child; through blood transfusion, bone marrow transplants, or organ transplants, and sexual transmission [5]. 

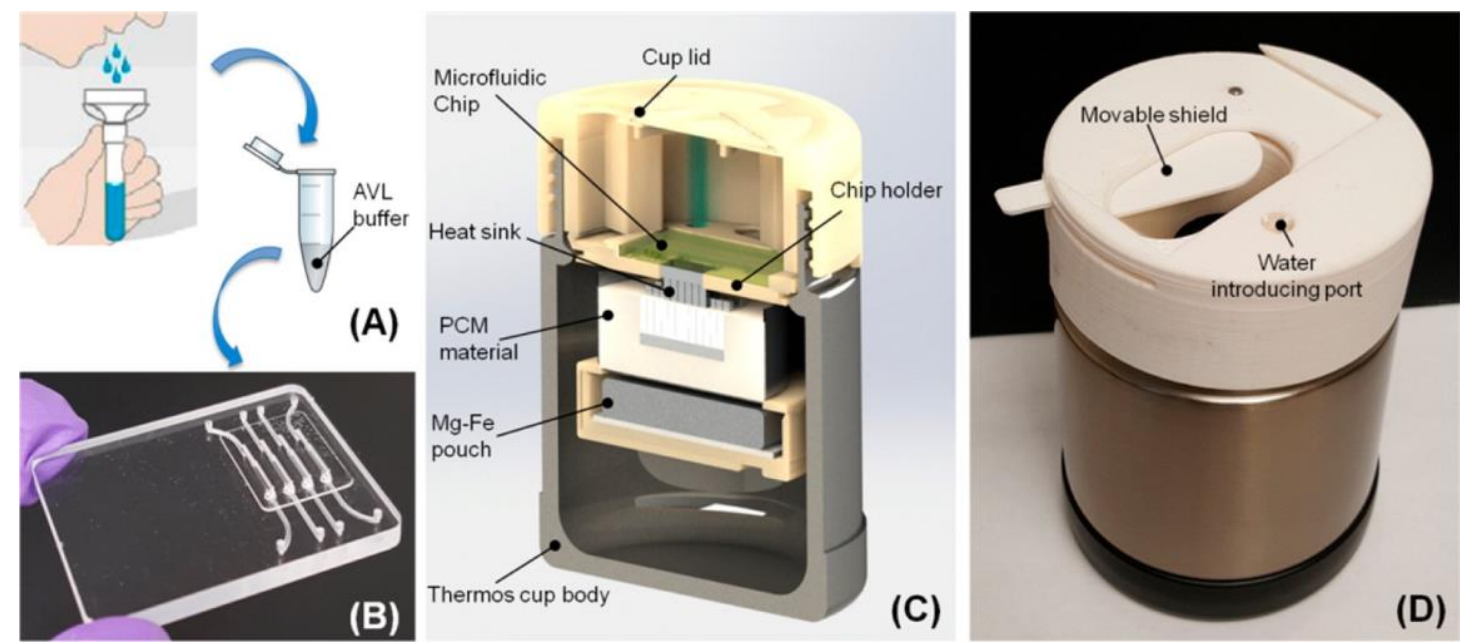

Figure7.2 Point-of-care molecular detector. a) Schematic of saliva sample preparation. Saliva samples are first collected in a saliva collection tube and then lysed in Qiagen binding/ lysis (AVL) buffer. b) The lysed sample is filtered through the isolation membrane of our microfluidic cassette for nucleic acid extraction. c) Exploded view of the chemically heated cup. The cup consists of a thermos cup body, a 3D-printed cup lid, a chip holder, PCM material, heat sink and single-use $\mathrm{Mg}-\mathrm{Fe}$ alloy pack heat source. d) A photograph of the chemically heated cup for point of care molecular diagnostics of ZIKV, obtained with permissions from Ref. [11].

As a major and fundamental infection spread, horizontal transmission, that is, from mother to child during pregnancy, gives rise to microcephaly in newborns and also other birth defects [6]. Several approaches have been introduced and conducted for practical detection of this type of infection such as reverse transcriptase-polymerase chain reaction [7], antibody-based methods (e.g. ELISA) [7], point-of-care molecular detection [8], and electrochemical biosensing [9]. Despite of the growing research, most of these applications suffer from high-costs, lack of sensitivity and repeatability, and complex processing. Therefore, providing an all-optical microscale metasurface with an ability to detect picomolar concentrations of ZIKV envelope protein would help us to tailor 


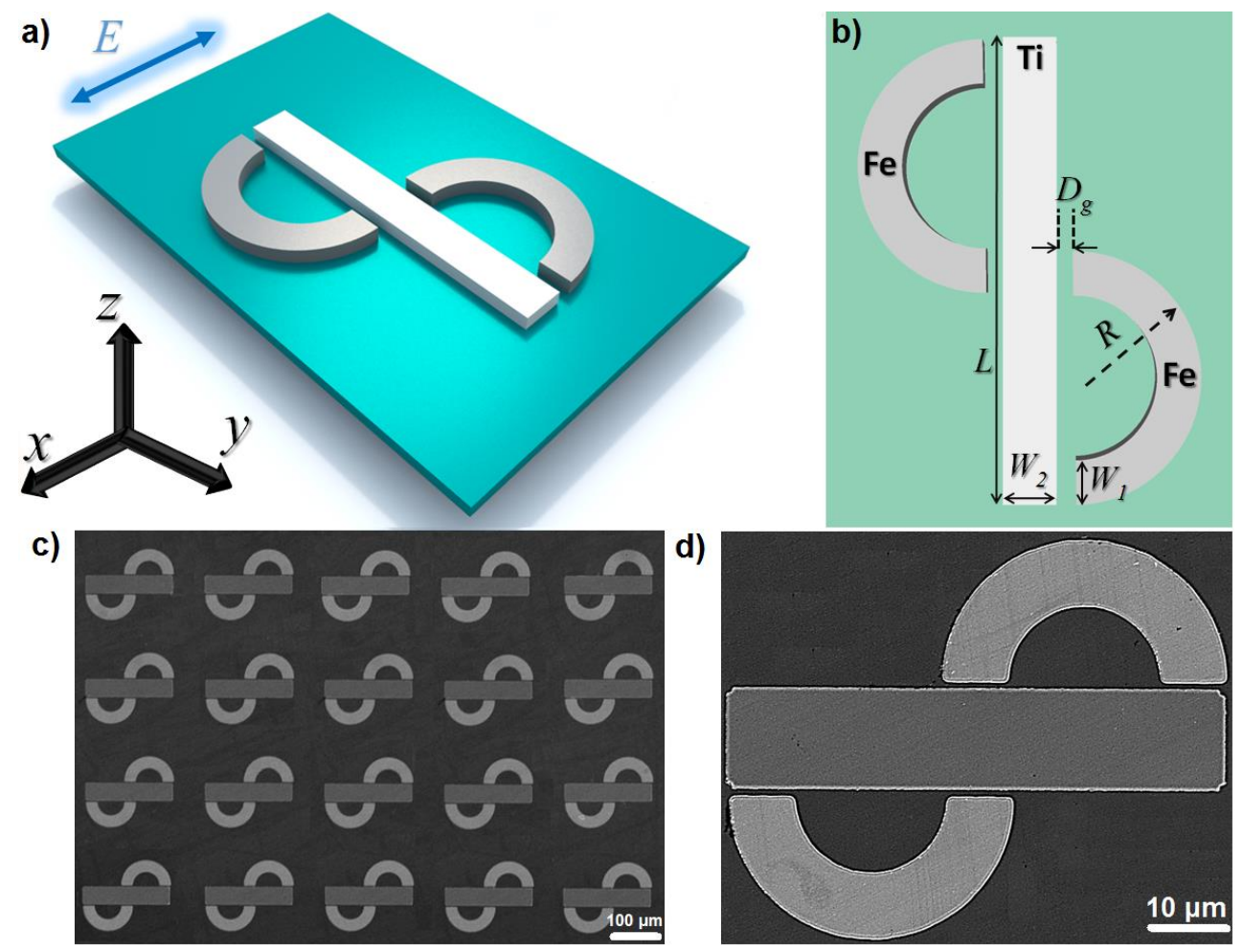

Figure 7.3 Toroidal plasmonic metamolecule. a) Artistic perspective of compositional plasmonic resonators assembly as a unit cell on a silicon host. b) A top-view schematic of the microstructure unit cell with an introduction to geometrical components. c) The SEM image of fabricated plasmonic structures in arrays for the unit cells with the gap spots between surrounding and central resonators of $D_{g}=3 \mu \mathrm{m}$ with $L=240 \mu \mathrm{m}, R=50 \mu \mathrm{m}, W_{l}=30$ $\mu \mathrm{m}$, and $W_{2}=40 \mu \mathrm{m}$. d) The magnified SEM images for each unit cell with $D_{g}=3 \mu \mathrm{m}$.

practical, easy to fabricate, and accurate detection mechanism with high reliability. Figure 7.1a illustrates an art picture of the ZIKV virion structure with the description to various components of the molecular protein. The cryo-electron microscopic crosssectional image of frozen hydrated ZIKV is plotted in Fig.7.1b [10], showing the color coding of the virus based on radii as follows: blue, up to $130 \AA$; cyan, 131 to $150 \AA$; green, 151 to $190 \AA$; yellow, 191 to $230 \AA$; red, $231 \AA$ and above. The region shown in blue fails to follow icosahedral symmetry, and therefore its density is uninterruptable, as is the case with other flaviviruses. 
As we described above, so far, various detection method have been carried out to increase the sensitivity of devices at very low concentrations of ZIKV agents. Newly, a sensing system has been developed based on highly sensitive reverse-transcription loopmediated, isothermal amplification assay for rapid detection of ZIKV and its implementation in a simple, handy, cost-effective, point-of-care disposable cassette that carries out all the unit operations from sample introduction to detection [11]. The thermal control of the cassette was established by using a chemically heated cup without a need for electrical power. Figure 7.2 exhibits the illustrations of the proposed ZIKV sensor by Song et al. [11]. The operating mechanism of this sensors is based on spiking saliva samples with varying concentration of ZIKV infection. Then, a colorimetric detection system was carried out based on including leuco crystal violet (LCV) dye with the associated reaction mixture. Once the infectious virus is detected, the colorless LCV turn into violet. Although this method looks promising and fast, it suffers from poor detection performance at very low concentrations of ZIKV agents, which is around a few micromolar $(\mu \mathrm{M})$. Therefore, finding an approach to detect low level of infectious agents would help to attain early-stage identification of disease. In the upcoming section, we describe our all-optical method for the detection of ZIKV envelope proteins at very low concentrations using $\mathrm{THz}$ plasmonic metamaterials.

\subsection{Tailoring Bimetallic Multipixel Toroidal Unit Cells}

Here, we employed the analyzed plasmonic metasurface that is investigated in Chapter 5 in details. Using both numerical and experimental studies, we verified the excitation of strong and high-quality toroidal moments in multimetallic plasmonic metamolecules in the $\mathrm{THz}$ regime. In the following subsections, we briefly summarize the spectral 
properties and toroidal response of the developed metadevice and then the unique advantages of the multimetallic metasurface for the detection of ZIKV envelope proteins will be discussed comprehensively.

\subsubsection{The Spectral Properties}

Figure 7.3 demonstrates the schematic view of the proposed planar micro-assembly unit on a silicon host (not to scale) with the incident $\mathrm{THz}$ beam direction and electric field polarization. The geometrical and material descriptions of the resonators and components are demonstrated in a top-view profile in Fig. 7.3b. Figure 7.3c exhibits an SEM image of the fabricated compositional unit cell arrays on a high-resistivity silicon wafer with the gap distance of $D_{g}=3 \mu \mathrm{m}$ between peripheral and central resonators. The magnified SEM image of the planar plasmonic unit cell is presented in Fig. 6.3d. In numerical analysis, we assumed formation of a few nanometers natural oxide $\left(\mathrm{Fe}_{2} \mathrm{O}_{3}\right)$ on the Fe structures at room temperature [12].

By launching a THz beam in $-z$ direction (Fig. 7.3a), the excited local modes lead to formation of circular magnetic fields in the central zone of the peripheral curved structures. This results in dramatic suppression in the electric dipole moment by the excited magnetic and toroidal resonances [13]. The suppressed dipolar moment is associated with the strong electric resonant mode arising from the central resonator and the weak modes in the curved split resonators. Looking at the magnetic resonance $(\mathbf{m})$ direction in the upper and lower parts of the magnetic split resonators (Fig. 7.4a, not to scale) and central block, strong magnetic fields oscillate in antiphase regime, while the excited weaker magnetic modes at the central resonator acts as an in-phase component [14]. On the other hand, Fig. 76.4b illustrates formation of a head-to-tail configuration of 
the magnetic moments leading to a toroidal dipolar moment $(\mathbf{T})$ at the center of the unit cell created by the currents $(\mathbf{j})$ on the surface of a torus along the circular meridian. The arrows show the current flux direction and magnetic moment (m) oscillation as a closeloop arrangement inside the profile. Obviously, the head-to-tail configuration is performed with $90^{\circ}$ angle to the central block due to antisymmetric geometry of the unit cell. The corresponding transmitted magnetic radiation from the magnetoplasmonic unit cells arrays can be obtained by taking the summation of the scattered magnetic and incident electromagnetic fields. The total contribution of the far-field scattering of the magnetic field $\left(H_{\text {scat }}\right)$ can be written as [15]:

$$
H_{\text {scat }}=\frac{k^{2}}{Z_{0} 4 \pi \varepsilon_{0}}\left[\left(\left[\left(\mathbf{n} \times \mathbf{m}_{\mathbf{c}}\right) \times \mathbf{n}+i k \mathbf{n} \times \mathbf{T}_{\mathbf{c}} \times \mathbf{n}\right]\right)\right] \times \mathbf{n}
$$

where $k$ is the wave vector, $Z_{0}$ is the impedance of the medium, $\varepsilon_{0}$ is the permittivity of the vacuum, $\mathrm{n}$ is a unit vector in the direction of the incident illumination, and finally, $\mathrm{m}_{\mathrm{c}}$ and $T_{c}$ are the magnetic and toroidal dipolar moments, respectively, defined as [16]:

$$
\left\{\begin{array}{l}
\mathbf{m}_{\mathbf{c}}=\frac{1}{2 c} \int(\mathbf{r} \times \mathbf{J}) d^{3} \mathbf{r} \\
\mathbf{T}_{\mathbf{c}}=\frac{1}{10 c} \int\left[(\mathbf{r} . \mathbf{J}) \mathbf{r}-2 r^{2} \mathbf{J}\right] d^{3} \mathbf{r}
\end{array}\right.
$$

where $\mathbf{J}$ is the induced current density over the entire volume of the area and $c$ is the conventional speed of the light. To show the strong dependence of the magnetic response to the geometrical parameters, we first analyze the effect of the offset gaps between the peripheral and central resonators on the electromagnetic response as shown in Figs.7.4c(i)-7.4e(i). These analysis would help us to control the position and sharpness of the induced magnetoplasmonic resonances by varying the offset gap. A sharp magnetic 188 

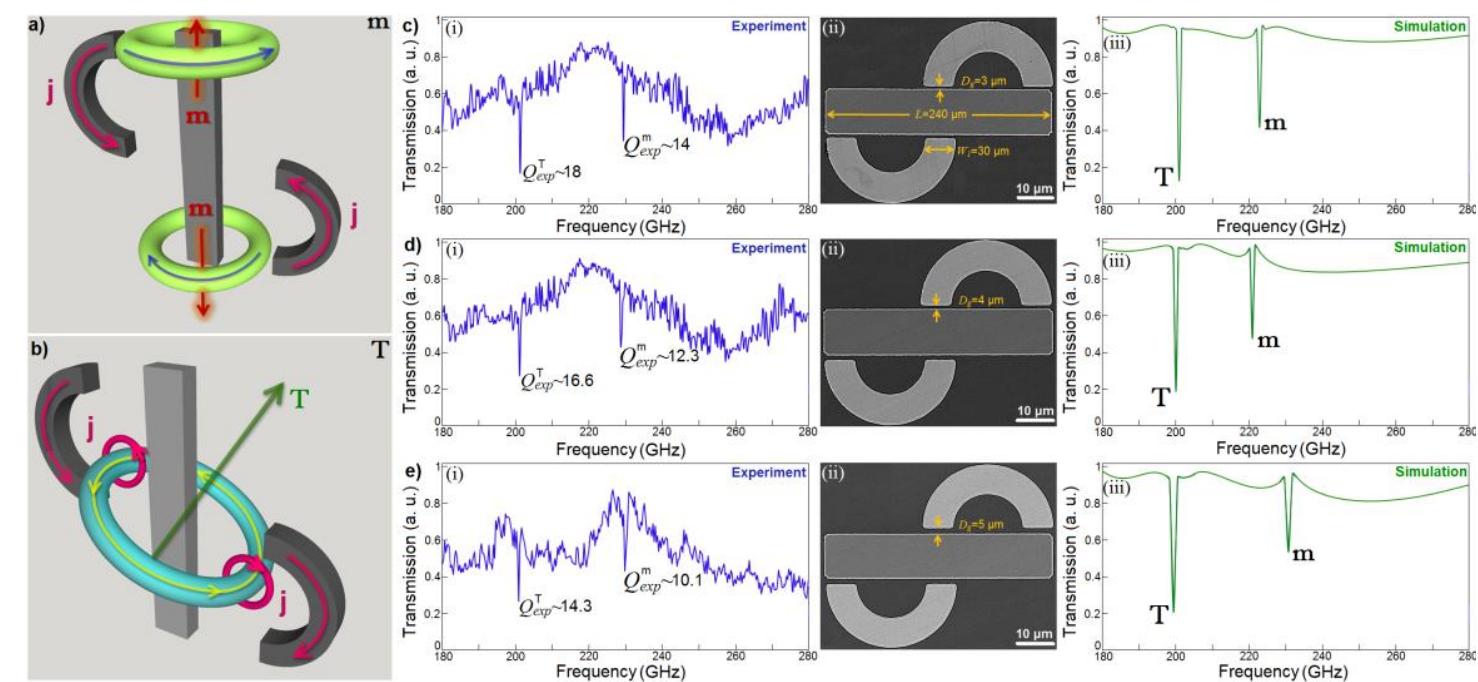

Figure 7.4. a) and b) The 3D schematics of the magnetic (m) and toroidal (T) resonances around and across the central and surrounding magnetic resonators, respectively. c), d), and e) The electromagnetic response of the compositional $\mathrm{THz}$ plasmonic resonator: (i) Experimentally obtained normalized transmission amplitude profiles for the unit cells with three different offset gaps, (ii) the SEM images for different offset gaps between resonators, (iii) Numerically calculated transmission spectra for three different offset gaps.

dipolar minimum is observed at $\sim 230 \mathrm{GHz}$ (indicated by $\mathbf{m}$ in Fig. $7.4 \mathrm{c}$ ) in the experimentally measured normalized transmission amplitude profile for $D_{g}=3 \mu \mathrm{m}$ which is attributed to an in-phase magnetic mode. On the other hand, an ultrasharp and distinct lineshape is induced at $\sim 203 \mathrm{GHz}$ correlating with the magnetic toroidal dipole (T). At this point, the induced magnetic fields in the satellite split resonators and the close-loop magnetic moment at the offset gap area (the point that both resonators meet each other) cause to formation of a head-to-tail configuration of the magnetic dipoles via suppression of the classical modes in a similar fashion that has been reported for 3D structures 


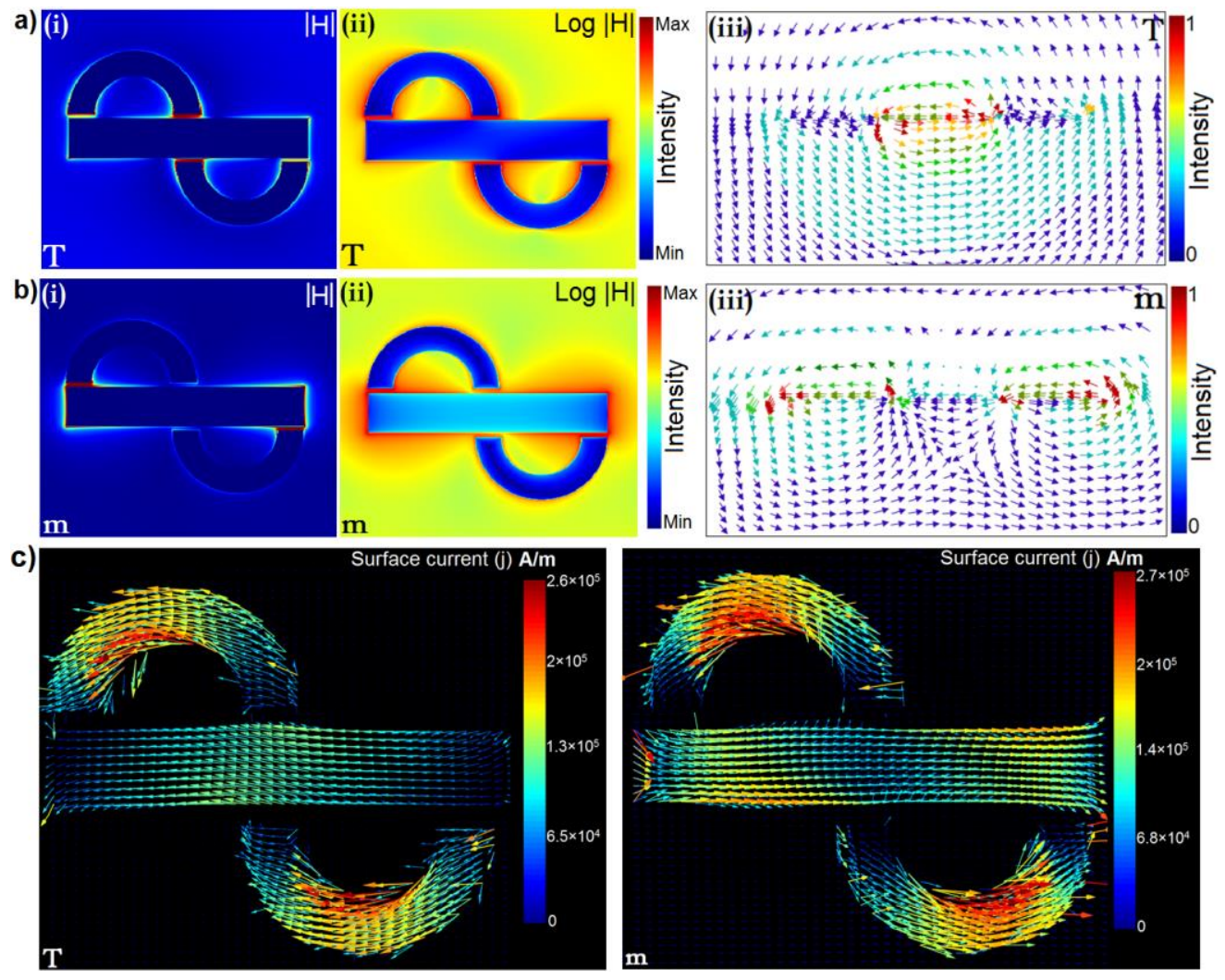

Figure 7.5. The electromagnetic response of the plasmonic unit cell at a) toroidal and $b$ ) magnetic resonance frequencies. Numerically obtained local $|\mathrm{H}|$-field $(\mathrm{A} / \mathrm{m})$ snapshots for the toroidal and magnetic resonance confinement and excitation at the gap spot area between resonators in (i) linear and (ii) logarithmic scales. (iii) The cross-sectional vectorial maps for the magnetic field lines at the position of toroidal and magnetic resonant modes. c) Simulated surface currents $(\mathrm{j})$ of unit cell at toroidal and magnetic resonances.

$[16,17]$. One should note that inducing such a distinct and pronounced toroidal magnetic moment using conventional planar structures is a serious challenge. Our tailored plasmonic unit cell has an exquisite geometrical asymmetry which is enhanced by using two different materials. Presence of Fe resonators with high magnetic moment and plasmonic properties helps to formation of giant magnetic current around the middle $\mathrm{Ti}$ rectangle. The good electric and poor magnetic responses of the central Ti block help to prevent destructive interference of the strong magnetic moments arising from peripheral magnetic resonators with the moments from the middle rectangle. As a result, formation 
of a closed-loop head-to-tail magnetic moment configuration would be possible around the central part of the unit cell. Furthermore, the presence of the substrate below the planar unit cell resonator increases the asymmetry of the entire metasurface. In this regime, formation of multipolar magnetic and electric modes is feasible, however, these modes are not resonant at the toroidal frequency and cannot be observed in the transmission spectrum. By increasing the gap distance between the proximal resonators to $4 \mu \mathrm{m}$ and $5 \mu \mathrm{m}$ homogenously, we observed a trivial broadening in the linewidth and suppression of the toroidal dip which dramatically affected the quality-factor of both magnetic and toroidal modes (Figs. 7.4d(i) and 7.4e(i)). Such a trend can be better understood by analyzing the effect of the offset gap on the circulating had-to-tail toroidal mode. In fact, for larger offset gaps $\left(D_{g}>4 \mu \mathrm{m}\right)$, the excited magnetic field which contributes to formation of the circulating current becomes weaker, causing a huge mismatch between the induced electromagnetic currents in the peripheral and central resonators. The SEM images for the gap spot variations between Fe and Ti resonators in unit cells are shown in Figs. 7.4(ii)-7.4e(ii). The experimentally obtained results are in perfect agreement with the simulation predictions (see Figs 7.4c(iii)-7.4e(iii)). We calculated the corresponding experimental quality-factors as high as $Q_{\exp }^{\mathrm{m}}=14$ and $Q_{\exp }^{\mathrm{T}}$ $=18$ for the magnetic and toroidal modes, respectively, using the highest peak and lowest minimum of the induced toroidal dipolar dip. Achieving such a high narrow toroidal lineshape by a planar metasurface is the direct result of the strong magnetic resonance confinement and weak free-space coupling.

Figures $7.5 \mathrm{a}$ and $7.5 \mathrm{~b}$ exhibit the numerically calculated local magnetic field $(|\mathrm{H}|)$ localization in a standalone unit cell resonator, showing the intense magnetic field 

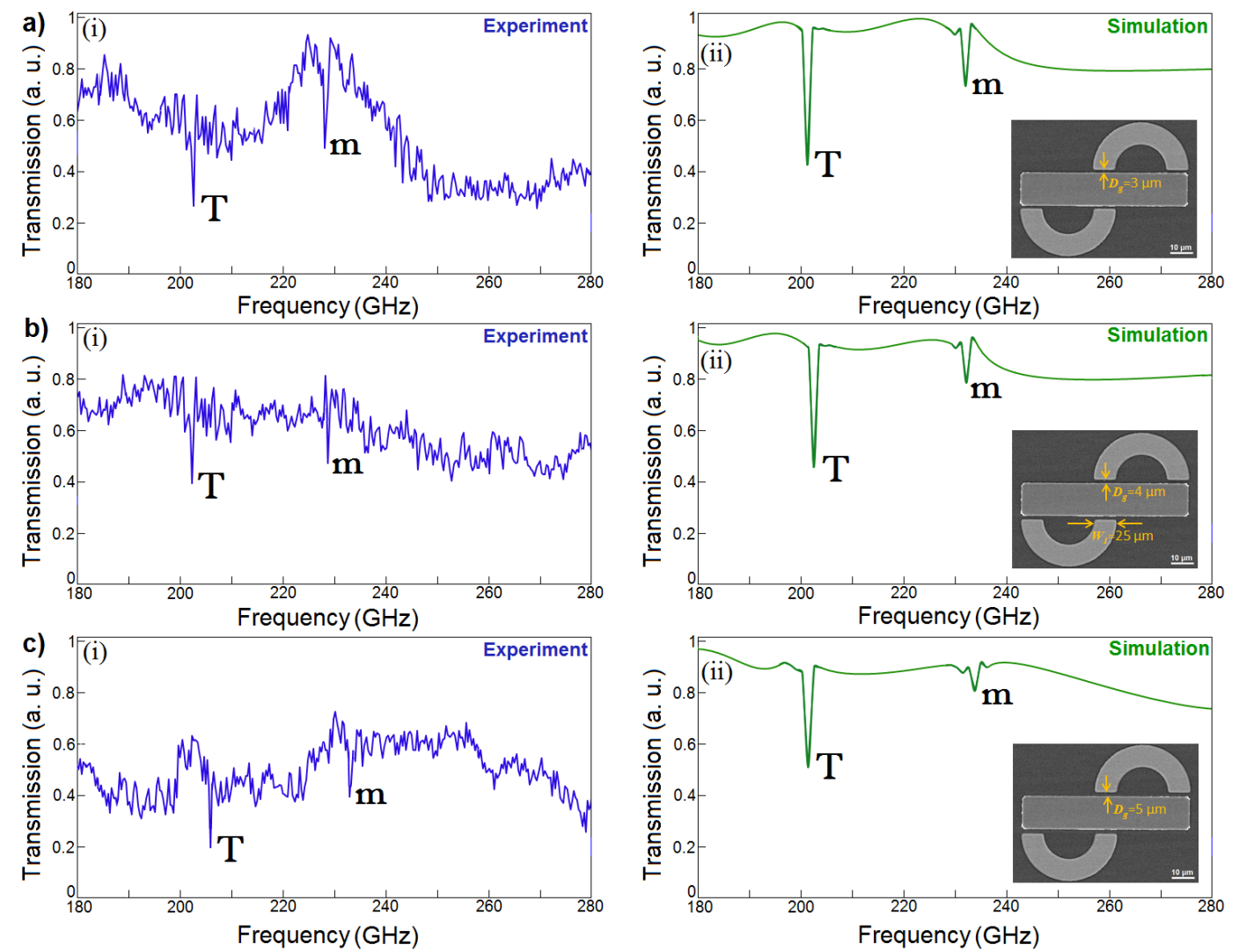

Figure 7.6. Normalized transmission amplitude profiles of the THz resonator system with three different offset gaps obtained (i) experimentally and (ii) numerically for (a) $D_{g}=3 \mu \mathrm{m}$, (b) $D_{g}=4 \mu \mathrm{m}$, and (c) $D_{g}=5 \mu \mathrm{m}$. The insets are the SEM images with the geometrical parameters.

confinement at the center of the antenna at the toroidal and magnetic frequencies, respectively in both linear and logarithmic scales. In addition, we demonstrated the crosssectional panels for the magnetic field (H-field) excitation across the plasmonic unit cell at both toroidal and magnetic resonant moments as shown in Figs. 7.5a(iii) and 7.5b(iii), respectively. These planes provide a better view of the magnetic field disturbance due to formation of heat-to-tail circular magnetic fields at the center of the unit cell. The surface current (j) also simulated for both resonant modes as shown in Fig. 7.5c.

In continue, we analyzed the effect of the geometrical variations in the magnetic peripheral curved resonators on the plasmonic response of the metasurface. To this end, 
by keeping the width of the central block fixed at $W_{2}=40 \mu \mathrm{m}$, we reduced the widths of the satellite resonators to $W_{l}=25 \mu \mathrm{m}$ with the radii fixed to $R=50 \mu \mathrm{m}$. Figure 7.6 shows both simulation and experimental results for three different gaps. With the reducing width of the magnetic components strength of the magnetic dipole moment (m) decays dramatically and does not radiate as strong as it does in the previous cases. Therefore, a significant decay is expected in the oscillating magnetic field around the central block (toroidal mode) due to dominant behavior of the excited classical electric dipolar and multipolar moments. It should be noted that despite of possessing prevailing response, both electric and magnetic multipolar moments are not still resonant in this frequency due to poor scattering efficiency [18]. Comparing Fig. 7.6a and Fig. 7.4a, the significant decay in the corresponding quality-factor of the toroidal mode is clear. In the same way, the magnetic dipole moment also decays dramatically due to both electric and magnetic classical multipolar modes' dominancy. In this limit, increasing the gap distance between the central and peripheral resonators gives rise to continuing decay in the quality factor of both induced modes (see Figs. 7.6b and 7.6c). For $D_{g}=5 \mu \mathrm{m}$, the magnetic dipolar moment almost disappears and hard to identify in the experiments. The minor blue-shift in the positions of both resonant dips is attributed to the geometrical variations, which can be described by Mie scattering theory [17,19]. The insets in simulation profiles (Figs. 7.6a(ii)-7.6c(ii)) are the SEM images of the studied geometrical variations.

\subsubsection{The Detection Performance}

As a promising technique, $\mathrm{THz}$ spectroscopy allows for non-invasive, non-contact, nondestructive, and label-free biomarker detection and therefore attracted growing interest for biomedical and clinical applications [20-23]. It is shown that electromagnetic field 

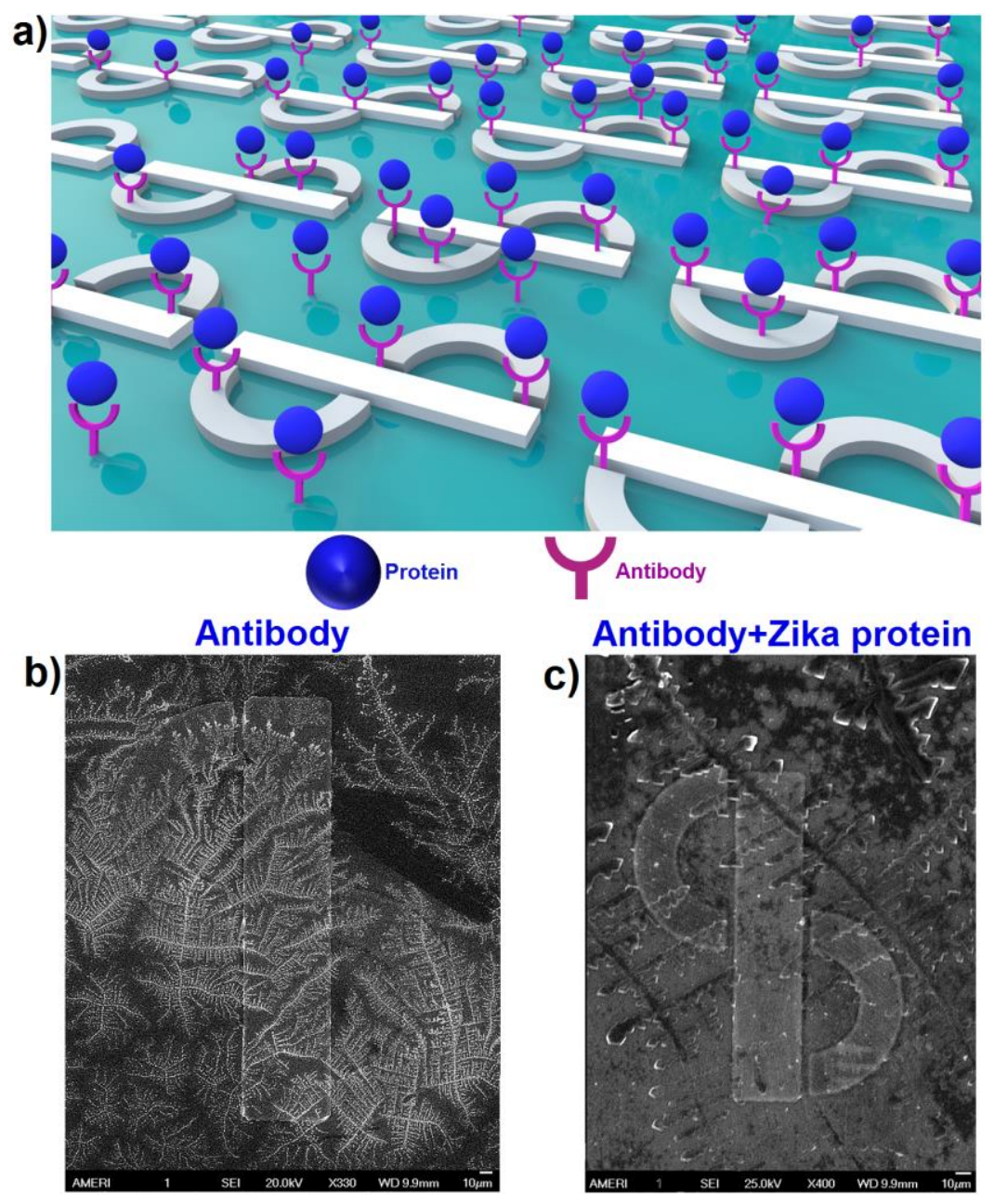

Figure 7.7. a) Schematic demonstration of ZIKV envelope protein binding with respective antibody on the toroidal $\mathrm{THz}$ plasmonic metasurface. b), and c) The SEM images for the plasmonic toroidal resonator covered with antibody and ZIKV envelope proteins attached to the antibody, respectively.

enhancement and confinement by metallic $\mathrm{THz}$ components facilitate detection of targeted bio-agents such as specific proteins, antibodies, and etc. [20-23,24]. Despite of such a unique potential, the selectivity and sensitivity of $\mathrm{THz}$ metasurfaces for immunosensing sensing applications have not been analyzed comprehensively due to mismatch between resonance frequency of nanoscale bio-targets and metasurfaces. This is because of non-responsivity of micro- and nano-organisms with the size of 
approximately $\sim \lambda / 100$, causing to be almost transparent to the incident radiation, therefore, reflect poor scattering cross-section [25]. This challenge in $\mathrm{THz}$ metamaterials can be circumvented using two approaches: 1) introducing nanosize particles (e.g. nanospheres) [25] on the micro-scale plasmonic chips to trap and bind biological objects and monitor their effect on the spectral response, and 2) excitation of ultrasharp antisymmetric resonances (with high-quality-factors) to show super-sensitivity to the small environmental variations.

The unique electromagnetic response of the studied $\mathrm{THz}$ structure can be used to tailor highly sensitive and accurate plasmonic sensor. To this end, we prepared series of chips with the best quality-factor (with the following geometrical parameters: $D_{g}=3 \mu \mathrm{m}, L=240$ $\mu \mathrm{m}, R=50 \mu \mathrm{m}, W_{l}=30 \mu \mathrm{m}$, and $\left.W_{2}=40 \mu \mathrm{m}\right)$ to achieve precise sensing. The immunosensing samples were prepared in three different configurations: 1) with antibody, 2) with antibody and bovine serum albumin (BSA), and 3) with antibody, bovine serum albumin (BSA), and variant concentration $\left(1 \mathrm{pg} / \mathrm{mL}\right.$ to $\left.10^{4} \mathrm{pg} / \mathrm{mL}\right)$ of immobilized ZIKV envelope protein. To prepare the samples for the fingerprint biological assay, we used both lyophilized 99\% bovine serum albumin (BSA) purchased from Sigma-Aldrich, and $\mathrm{pH} 7.4$ phosphate buffer solution (PBS) to dissolve the immunoreagents. The antibody and envelope proteins were purified by Diethylaminoethyl (DEAE) column chromatography and presented in $0.015 \mathrm{M}$ potassium phosphate $\left(\mathrm{KH}_{2} \mathrm{PO}_{4}\right)$ and $0.85 \%$ of $\mathrm{NaCl}$ with the $\mathrm{pH}$ around $\sim 7.2$. For preparing the samples for real-time characterization, $10 \mu \mathrm{L}$ of Zika antibodies $(1 \mathrm{mg} / \mathrm{mL})$ in PBS were locally deposited on the sensing area of $\mathrm{THz}$ structures and incubated for $15 \mathrm{~min}$. 
After washing the chips with PBS, antibody-modified structures were incubated in PBS containing 0.1 wt. \% BSA for $15 \mathrm{~min}$, and then, in a solution of a recombinant Zika diluted in PBS for at least 20 min (The ZIKV envelope protein concentration was ranged from 1 to $10^{4} \mathrm{pg} / \mathrm{mL}$ ). Here, the recombinant of ZIKV envelope protein is an artificial ZIKV protein created through genetic engineering process (recombinant DNA technology). The recombinant ZIKA envelope protein in our research was purchased from Sino biological USA. Once prepared, antibody-functionalized microstructures were rinsed and stored at $4{ }^{\circ} \mathrm{C}$ until used. The mouse monoclonal antibody for ZIKV and the envelope proteins purchased from Aalto Bio Reagents and Sino Biological Inc. respectively.

Figure 7.7a shows an artistic picture for the proposed metasurface with the presence of antibody and trapped envelope proteins around and on the plasmonic resonators. Figures $7.7 \mathrm{~b}$ and $7.7 \mathrm{c}$ are the SEM images of the presence of immobilized ZIKV antibody on a sample metallic microstructure and a chip covered with antibody-attached ZIKV envelope protein, respectively. These images helps to understand the binding quality of antibody and capturing of biomarker proteins to the bimetallic metamolecules directly right after the deposition in the metasurface. Figures 7.8(i) and 7.8(ii) illustrate the transmission spectra of the plasmonic metasurface for different concentrations of ZIKV envelope protein captured by the antibody. By focusing on the behavior of magnetic toroidal mode, we observed a prominent resonance in the presence of ZIKV envelope protein concentration between $1 \mathrm{pg} / \mathrm{mL}$ to $10^{4} \mathrm{pg} / \mathrm{mL}$. In the earlier section, we observed excitation of the toroidal resonance mode at $203 \mathrm{GHz}$ for the bare resonators. In the presence of the ZIKV antibody, the toroidal mode remained unchanged at $203 \mathrm{GHz}$ 

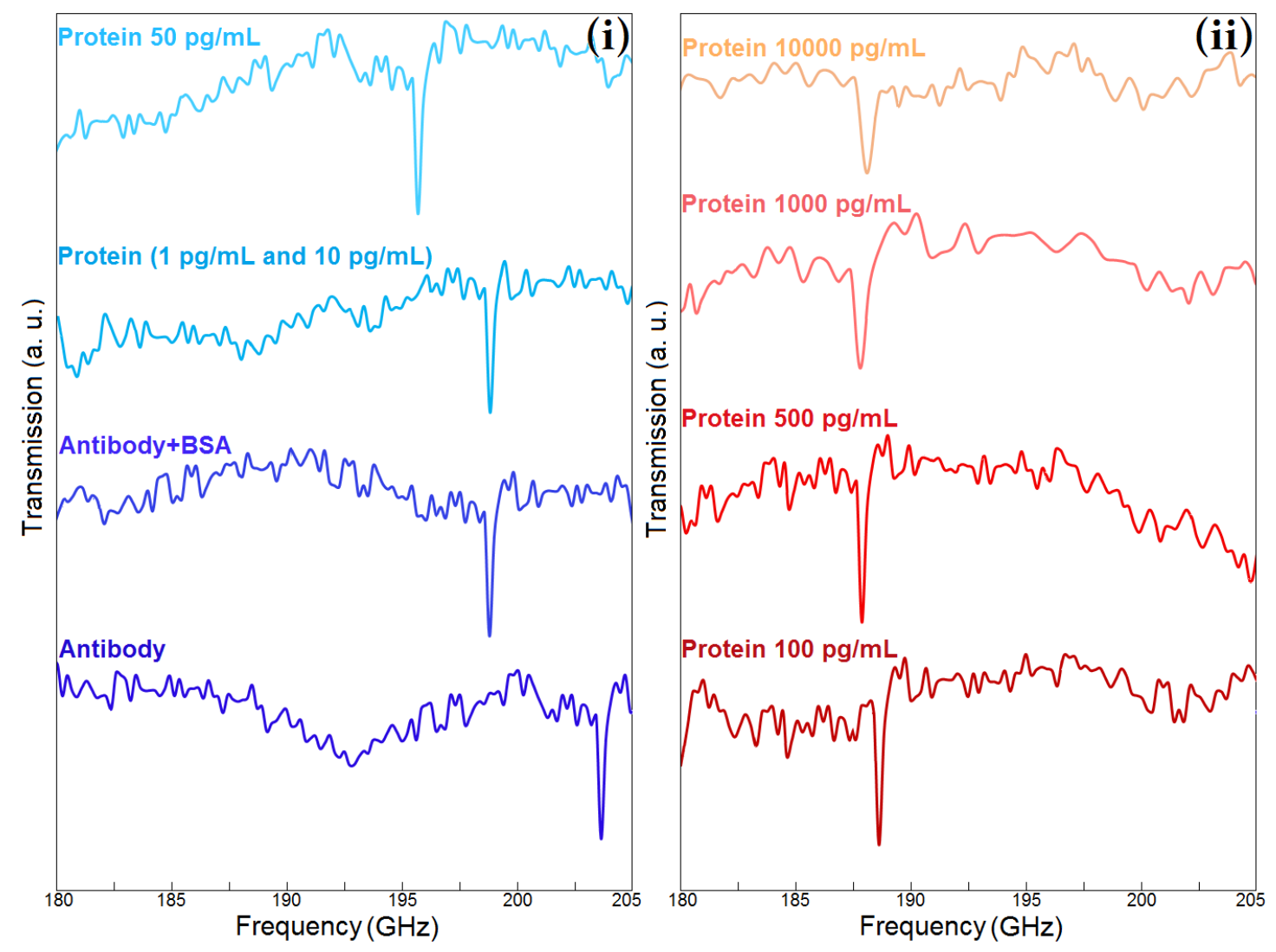

Figure 7.8. Transmission spectra for the toroidal resonant mode behavior for presence of different concentration of ZIKV envelope protein from (i) antibody to $50 \mathrm{pg} / \mathrm{mL}$ and (ii) 100 $\mathrm{pg} / \mathrm{mL}$ to $10^{4} \mathrm{pg} / \mathrm{mL}$.

due to its optically non-responsiveness to the incident radiation. For the solution composed of ZIKV antibody plus BSA the magnetic toroid moment red-shifted to 198 GHz. This is because of formation of a layer on top of the plasmonic sensing device, which affects the entire refractive index of the surrounding ambience and shifting the toroid moment. It should be underlined that the presence of BSA layer helps to improve ZIKV envelope protein capturing by respective antibody effectively and preventing nonspecific binding of ZIKV envelope protein. Adding $1 \mathrm{pg} / \mathrm{mL}$ and $10 \mathrm{pg} / \mathrm{mL}$ of the target protein did not cause any shift in the position of the toroidal moment, and it remained at $198 \mathrm{GHz}$. However, increasing the concentration to $50 \mathrm{pg} / \mathrm{mL}$ and $100 \mathrm{pg} / \mathrm{mL}$, shifted the 
toroidal resonance to $194 \mathrm{GHz}$ and $188 \mathrm{GHz}$, respectively. Such a large shift in the resonance frequency shows the sensitivity of the toroid dip to the concentration of the infection protein. Interestingly, the narrowness and sharpness of the dipolar toroidal moment is almost unchanged, which helps keeping the sensing precision high by keeping the quality-factor high. This is unusual compared to classical plasmonic biosensing systems operating based on antisymmetric resonant lineshapes such as Fano and EIT resonances where perturbation in the environmental refractive index or physical changes cause to destructive effects on the lineshape quality [26-28]. Such a decay in the quality of resonant modes is caused by their strong dependency on the morphological and geometrical perturbations affecting the spectral response dramatically. Conversely, Savinov et al. [29] have theoretically and experimentally verified that the quality of toroidal moment does not decrease by minor morphological variations. Further increases in the concentration of ZIKV envelope protein to $500 \mathrm{pg} / \mathrm{mL}$ leads to a shift of the position of the pronounced toroidal resonant dip to $187 \mathrm{GHz}$. In continue, by increasing the concentration of target protein to $10^{3} \mathrm{pg} / \mathrm{mL}$ and $10^{4} \mathrm{pg} / \mathrm{mL}$, we observed a drastic decay in the quality of toroidal mode in both cases. Figure 7.9 a presents the frequency shifts $(\mathrm{GHz})$ as a function of protein concentration $(\mathrm{pg} / \mathrm{mL})$. ZIKV envelope protein with the concentration ranging from $1 \mathrm{pg} / \mathrm{mL}$ to $10 \mathrm{pg} / \mathrm{mL}$ did not cause to a noticeable frequency shift, reflecting weak sensitivity. While for the concentration ranging from 50 $\mathrm{pg} / \mathrm{mL}$ to $500 \mathrm{pg} / \mathrm{mL}$ a significant red-shift in the frequency of the toroidal mode is recorded. In this study, the limit of detection (LOD) can be defined by: $\mathrm{LOD}=3(S D) / S$ [30], where " $S D$ " is the standard deviation of the frequency shift and " $S$ " is the slope of the fitting line (shown by the dashed line in Fig. 7.9a), and the LOD is quantified as $~ 24$ 

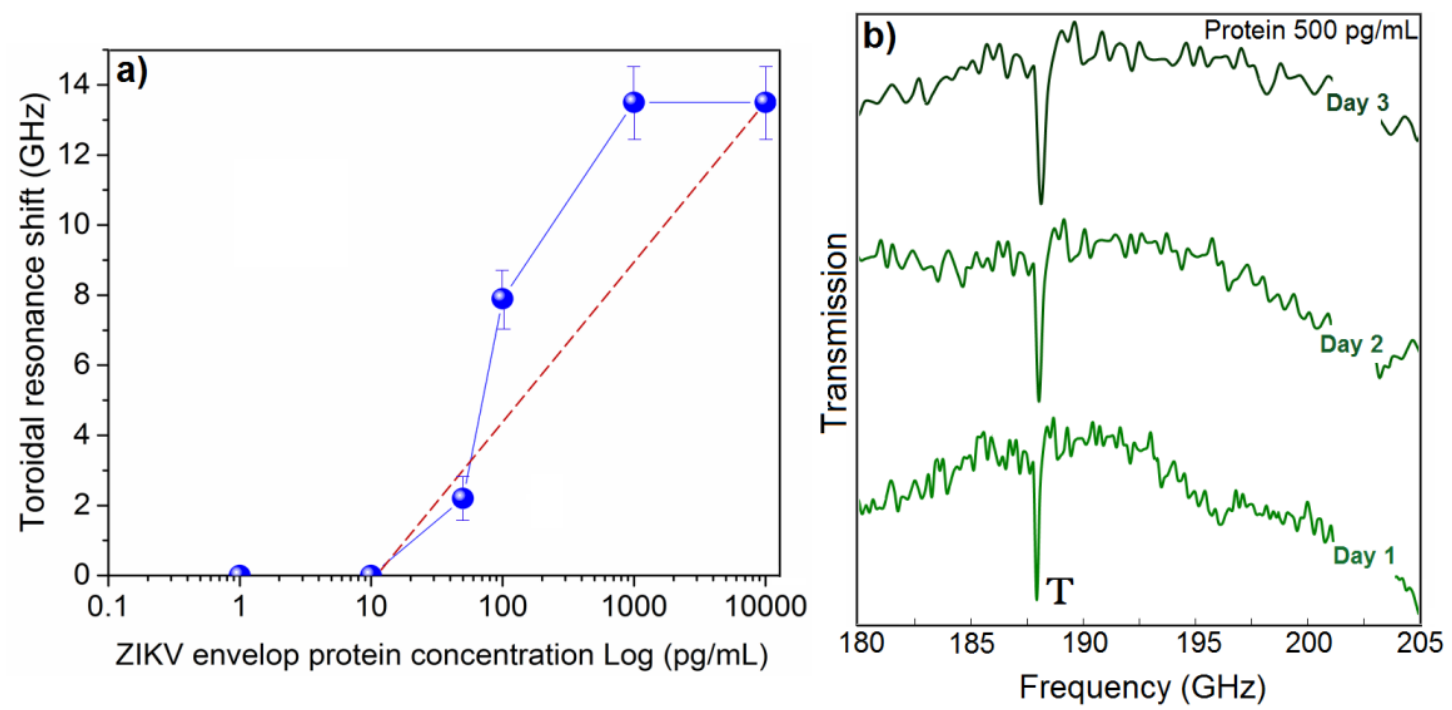

Figure 7.9. a) Toroidal resonance frequency shifts due to conjugated ZIKV protein concentration (solid) and fitting line (dotted). b) The transmission spectra for a $\mathrm{THz}$ plasmonic chip characterized for three days to define the repeatability of a sample.

$\mathrm{pg} / \mathrm{mL}$. By defining the slope of the toroidal position shift as a function of ZIKV envelope protein concentration, we estimated the sensitivity of the structure as 6.47 $\mathrm{GHz} / \log (\mathrm{pg} / \mathrm{mL})$.

We also analyzed the longevity and repeatability of the demonstrated $\mathrm{THz}$ plasmonic biosensors. To this end, samples with the antibody were prepared with the described technique above and stored at $4{ }^{\circ} \mathrm{C}$ before the measurements. Figure $7.9 \mathrm{~b}$ shows the measured transmission spectra for three consecutive days with the ZIKV concentration of $500 \mathrm{pg} / \mathrm{mL}$. The resonance quality remained excellent for three days. However, after this period of time, the toroidal dip became broader and dramatically damped. This deterioration also included a significant blue-shift in the position of magnetic resonant mode to the higher energies. Ultimately, we believe that the ability to identify low concentrations of a specific biomarker with low molecular-weight will be feasible by using the proposed metasensor. A comparison between newly reported $\mathrm{THz}$ plasmonic biosensing works, we facilitated detection of proteins with the molecular-weight of $\approx 13$ 
$\mathrm{kDa}$, while the recent achievements show detection of bio-objects with the weight of over $70 \mathrm{kDa}$ using THz biosensors.

\subsubsection{Conclusions}

Here, we have shown the excitation of ultrasharp toroidal dipoles in $\mathrm{THz}$ frequencies using bimetallic asymmetrical planar resonators. Using the magnetic nature of $\mathrm{Fe}$ and also the exotic geometrical design of the proposed structure, we achieved experimentally measured quality factor of 18 for the toroidal resonance. Taking advantage of the high quality toroidal moment resonance, we also demonstrated biosensing capability of the proposed structures. Spectral response of the samples loaded with the relevant antibody to the assays of ZIKV envelope protein with different concentrations shows that limit of detection of $\sim 24 \mathrm{pg} / \mathrm{mL}$ and $6.47 \mathrm{GHz} / \mathrm{log}(\mathrm{pg} / \mathrm{mL})$ is achievable. Further studies proved that the demonstrated biosensing platform could be reliable up to three days. The unique geometry of the proposed resonators also results in high polarization sensitivity which allows for their use in $\mathrm{THz}$ switching applications. Rapid detection capability combined with the very sharp resonance and easy fabrication of $\mathrm{THz}$ resonators compared to its counterparts in optical frequencies make the demonstrated devices promising platforms for biosensing purposes.

\subsection{References}

1. Musso, D., Roche, C., Nhan, T. X., Robin, E., Teissier, A., \& Cao-Lormeau, V. M. (2015). Detection of Zika virus in saliva. Journal of Clinical Virology, 68, 53-55.

2. Saxena, S. K., Elahi, A., Gadugu, S., \& Prasad, A. K. (2016). Zika virus outbreak: an overview of the experimental therapeutics and treatment. Virusdisease, 27(2), 111115.

3. Rasmussen, S. A., Jamieson, D. J., Honein, M. A., \& Petersen, L. R. (2016). Zika virus and birth defects-reviewing the evidence for causality. The New England Journal of Medicine 374, 1981-1987. 
4. Cugola, F. R., Fernandes, I. R., Russo, F. B., Freitas, B. C., Dias, J. L., Guimarães, K. P., Benazzato, C., Almeida, N., Pignatari, G. C., Romero, S., \& Polonio, C. M. (2016). The Brazilian Zika virus strain causes birth defects in experimental models. Nature, 534(7606), 267-271.

5. Bogoch, I. I., Brady, O. J., Kraemer, M. U., German, M., Creatore, M. I., Kulkarni, M. A., Brownstein, J. S., Mekaru, S. R., Hay, S. I., Groot, E., \& Watts, A. (2016). Anticipating the international spread of Zika virus from Brazil. Lancet, 387(10016), 335-336.

6. Hofer, U. (2016). Tracing the steps of Zika virus. Nature Reviews Microbiology, 14(7), 401-402.

7. Vorou, R. (2016). Letter to the editor: diagnostic challenges to be considered regarding Zika virus in the context of the presence of the vector Aedes albopictus in Europe. Eurosurveillance, 21(10), 30161.

8. Song, J., Mauk, M. G., Hackett, B. A., Cherry, S., Bau, H. H., \& Liu, C. (2016). Instrument-free point-of-care molecular detection of Zika virus. Analytical Chemistry, 88(14), 7289-7294.

9. Kaushik, A., Tiwari, S., Jayant, R. D., Vashist, A., Nikkhah-Moshaie, R., El-Hage, N., \& Nair, M. (2017). Electrochemical biosensors for early stage Zika diagnostics. Trends in Biotechnology, 35(4), 308-317.

10. Sirohi, D., Chen, Z., Sun, L., Klose, T., Pierson, T. C., Rossmann, M. G., \& Kuhn, R. J. (2016). The $3.8 \AA$ A resolution cryo-EM structure of Zika virus. Science, 352(6284), 467-470.

11. Song, J., Mauk, M. G., Hackett, B. A., Cherry, S., Bau, H. H., \& Liu, C. (2016). Instrument-free point-of-care molecular detection of Zika virus. Analytical Chemistry, 88(14), 7289-7294.

12. Wu, W., He, Q., \& Jiang, C. (2008). Magnetic iron oxide nanoparticles: synthesis and surface functionalization strategies. Nanoscale Research Letters, 3(11), 397.

13. Chen, X., \& Fan, W. (2017). Study of the interaction between graphene and planar terahertz metamaterial with toroidal dipolar resonance. Optics Letters, 42(10), 20342037. 
14. Wang, J., Fan, C., He, J., Ding, P., Liang, E., \& Xue, Q. (2013). Double Fano resonances due to interplay of electric and magnetic plasmon modes in planar plasmonic structure with high sensing sensitivity. Optics Express, 21(2), 2236-2244.

15. Jackson, J. D. (1999) Classical Electrodynamics. Wiley, New York.

16. Afanasiev, G. N., \& Dubovik, V. M. (1992). Electromagnetic properties of a toroidal solenoid. Journal of Physics A: Mathematical and General, 25(18), 4869.

17. Newton, R. G. (1982). Scattering Theory of Waves and Particles. Springer, New York.

18. Zayats, A. V. \& Maier, S. A. (2013) Active Plasmonics and Tuneable Plasmonic Metamaterials. Wiley, Danvers, MA.

19. Horvath, H. (2009). Gustav Mie and the scattering and absorption of light by particles: Historic developments and basics. Journal of Quantitative Spectroscopy and Radiative Transfer, 110(11), 787-799.

20. Smye, S. W., Chamberlain, J. M., Fitzgerald, A. J., \& Berry, E. (2001). The interaction between terahertz radiation and biological tissue. Physics in Medicine and Biology, 46(9), R101.

21. Mickan, S. P., Menikh, A., Liu, H., Mannella, C. A., MacColl, R., Abbott, D., Munch, J., \& Zhang, X. C. (2002). Label-free bioaffinity detection using terahertz technology. Physics in Medicine and Biology, 47(21), 3789.

22. Nagel, M., Först, M., \& Kurz, H. (2006). THz biosensing devices: fundamentals and technology. Journal of Physics: Condensed Matter, 18(18), S601.

23. Ahmadivand, A., Gerislioglu, B., Manickam, P., Kaushik, A., Bhansali, S., Nair, M., \& Pala, N. (2017). Rapid Detection of Infectious Envelope Proteins by Magnetoplasmonic Toroidal Metasensors. ACS sensors, 2(9), 1359-1368.

24. Tao, H., Strikwerda, A. C., Liu, M., Mondia, J. P., Ekmekci, E., Fan, K., Kaplan, D. L., Padilla, W. J., Zhang, X., Averitt, R. D. \& Omenetto, F. G. (2010). Performance enhancement of terahertz metamaterials on ultrathin substrates for sensing applications. Applied Physics Letters, 97(26), 261909. 
25. Ashkin, A., \& Dziedzic, J. M. (1987). Optical trapping and manipulation of viruses and bacteria. Science, 235, 1517-1521.

26. Kuznetsov, A. I., Evlyukhin, A. B., Gonçalves, M. R., Reinhardt, C., Koroleva, A., Arnedillo, M. L., Kiyan, R., Marti, O., \& Chichkov, B. N. (2011). Laser fabrication of large-scale nanoparticle arrays for sensing applications. ACS Nano, 5(6), 4843-4849.

27. Dong, Z. G., Liu, H., Cao, J. X., Li, T., Wang, S. M., Zhu, S. N., \& Zhang, X. (2010). Enhanced sensing performance by the plasmonic analog of electromagnetically induced transparency in active metamaterials. Applied Physics Letters, 97(11), 114101.

28. Meng, F. Y., Wu, Q., Erni, D., Wu, K., \& Lee, J. C. (2012). Polarization-independent metamaterial analog of electromagnetically induced transparency for a refractiveindex-based sensor. IEEE transactions on Microwave Theory and Techniques, 60(10), 3013-3022.

29. Savinov, V., Fedotov, V. A., \& Zheludev, N. I. (2014). Toroidal dipolar excitation and macroscopic electromagnetic properties of metamaterials. Physical Review B, 89(20), 205112.

30. Currie, L. A. (1999). Detection and quantification limits: origins and historical overview. Analytica Chimica Acta, 391(2), 127-134. 


\section{CHAPTER 8}

\section{Nanoparticles-Enhanced Terahertz Toroidal Metasurface for Biomarker Proteins Detection}

In previous chapters, the biosensing properties of a toroidal $\mathrm{THz}$ plasmonic metasurface were analyzed numerically and experimentally for the identification of ZIKV envelope proteins. Although the sensitivity of the proposed metasensor was significant, it suffers from poor repeatability due to weak binding of bio-agents and the artificial metamolecules. To address this shortcoming, for a given plasmonic toroidal unit cell, we enhanced its spectral properties, sensitivity, and repeatability for tracing ultralow concentration of biomarker molecules by introducing GNPs to the metasystem.

\subsection{The Influence of Gold Nanoparticles on Toroidal Momentum}

In this section and following subsections, we focus on the spectral properties of a toroidal metasensor and the influence of the presence of GNPs attached to the unit cells using both numerical and experimental analysis. Then, the developed metamaterial will be employed for ZIKV envelope proteins detection.

\subsubsection{The Spectral Properties}

Figure 8.1a demonstrates an artistic 3D rendering of the investigated planar toroidal gold unit cell composed of two symmetric proximal resonators with an offset capacitive gap in the curved arms. The important geometrical parameters of the plasmonic structure are given in Fig. 8.1b. As a critical component in the formation of toroidal momentum, we focused our electromagnetic and experimental analysis on the capacitive gaps ( $g$ ) 
variations by providing constant values for the other geometrical parameters as follows: $R_{i} / W / L / D=60 / 15 / 105 / 15 \mu \mathrm{m}$. The scanning electron microscope (SEM) image of the fabricated metamolecule arrays with the gap of $g=5 \mu \mathrm{m}$ is depicted in Fig. 8.1c. The schematic image of toroidal dipole (T) formation is shown in Fig. 8.1d, which is originated due to near-field interference of antiparallel magnetic moments arising from proximal resonators. The circulation of magnetic field around the parallel arms of the unit cell blocks is plotted with the direction of magnetic field (m) across the unit cell, verifying the formation of head-to-tail and closed-loop configuration of toroidal dipole that will be discussed later. It should be noted that the proposed unit cells are strongly polarization-dependent and the toroidal resonance can be excited only under $y$-polarized beam excitation, while for the $x$-polarized illumination the toroidal mode does not form. Figure 8.1e exhibits the utilized THz BWO setup, used for excitation and characterization

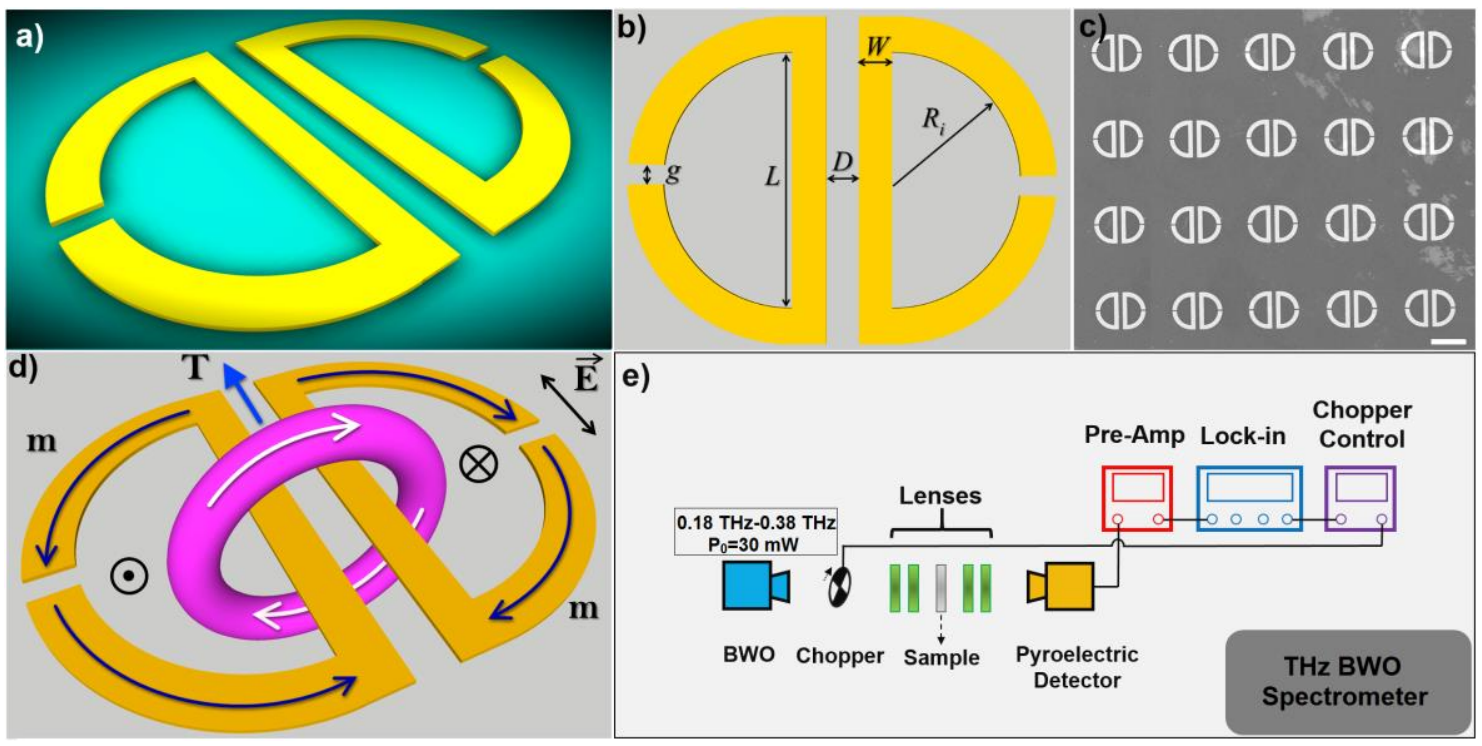

Figure 8.1. a) Artistic rendering of the toroidal unit cell. b) Geometrical parameters of the unit cell. c) SEM image of the fabricated metasurface. (d) Schematics of the formation of head-to-tail arrangement correlating with the toroidal momentum between the proximal resonators with the direction of magnetic momenta. e) A schematic representation of the BWO setup used to characterize the spectral response of the metasensor. 
of toroidal moments. For the fabrication of the proposed planar metasensors conventional single-level lithography based microfabrication process was developed. An undoped and high-resistivity silicon $(\mathrm{Si})$ wafer $(>10 \mathrm{k} \Omega . \mathrm{cm})$ with the crystal orientation of $<100>$ and thickness of $500 \mu \mathrm{m}$ was used as substrate. It was sonicated in acetone for $10 \mathrm{~min}$, and rinsed with isopropyl alcohol (IPA), deionized (DI) water, and dried by Nitrogen prior to the fabrication process. In continue, we deposited negative photoresists (NLOF 2020) with the thickness of $2.2 \mu \mathrm{m}$ and patterned intently in two different steps. Employing ebeam evaporation, we then deposited $50 \mathrm{~nm}$ of $\mathrm{Ti}$ and $300 \mathrm{~nm}$ of Au layers separately with the rate of $1 \AA / \mathrm{s}$ (99.99\% purity for $\mathrm{Ti}$ and $99.9995 \%$ purity for $\mathrm{Au}$, vacuum pressure $\sim 3.2 \times 10^{-4}$ mTorr). The Ti sublayer was used to enhance the adherence of gold layer to the surface of the $\mathrm{Si}$ wafer. The lift-off process was performed for 15 min by immersing the samples in acetone for $20 \mathrm{~min}$ at room-temperature using a sonication device, followed by IPA and DI water rinse. To characterize samples and extract the plasmon response of arrays with and without biological targets, a millimeter wave backward wave oscillator (BWO) setup combined with frequency multiplier (Microtech Instruments, Inc.) and broadband pyroelectric detector (Gentec Electro Optics Inc.) was operated at room-temperature. The spectral range of the incident radiation is between 100 $\mathrm{GHz}$ and $1.5 \mathrm{THz}$. The spectral resolution of the system is $10 \mathrm{MHz}$.

The proposed structure here is based on gold with the dielectric function experimentally obtained by Ordal et al. [1] for submillimeter wavelengths. By assuming the electric component (E) of the incident beam is parallel with the adjacent arms of unit cell ( $y$-polarized wave), and interacting with the planar metamolecules in the $z$-direction, we calculated and measured the transmission amplitude spectra in Figs. 8.2a-8.2c. These 

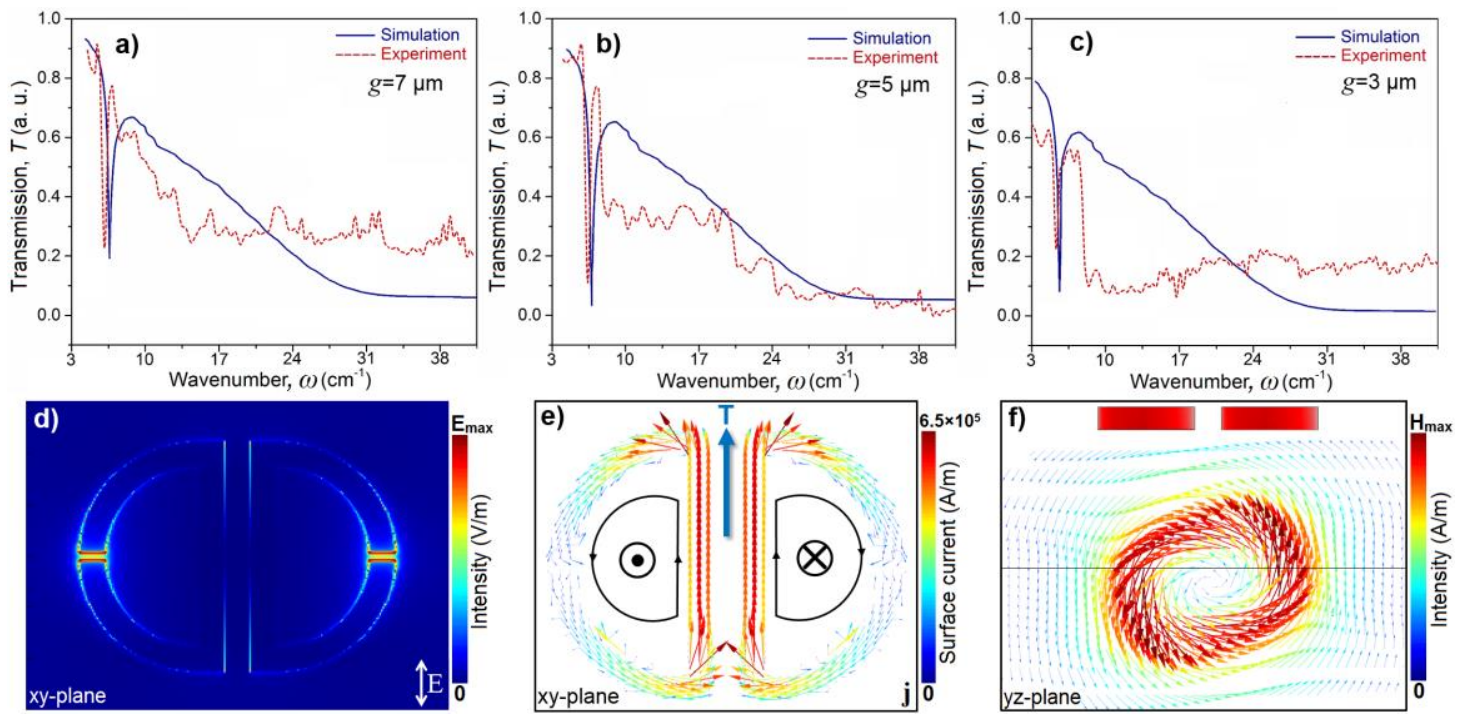

Figure 8.2. a), b), and c) Normalized transmission amplitude for the toroidal metamaterials with three different gap distances $g=7 \mu \mathrm{m}, 5 \mu \mathrm{m}$, and $3 \mu \mathrm{m}$, respectively. d) Local near-field map of the E-field enhancement at the gaps at the toroidal mode frequency. e) Surface current plot for the current across the structure and formation of toroidal mode. f) A cross-sectional yz-plane of the resonators, showing the head-to-tail magnetic moments forming the toroidal moment in a vectorial map.

profiles show the projected toroidal dipole momentum normal to the $z$-axis for varying capacitive gap sizes $(3 \mu \mathrm{m} \leq g \leq 7 \mu \mathrm{m})$ as a function of wavenumber, $\omega\left(\mathrm{cm}^{-1}\right)$. The toroidal resonance slightly red-shifted by decreasing the capacitive gap distance $(g)$. In all three regimes distinct and pronounced toroidal dipoles are excited in the transmission spectra, while the deepest and the most significant enhancement of toroidal dipole resonance is observed for $g=5 \mu \mathrm{m}$ around $\omega \approx 7 \mathrm{~cm}^{-1}$. There is an excellent agreement between the numerical analysis and the experimental measurements of the metasurface due to high resolution fabrication of the metamaterial. Theoretically, formation of coplanar loops of magnetic dipoles $(\mathbf{m})$ in proximal planar resonators that are oscillating oppositely leading to creating a platform for formation of toroidal multipoles (with the dominancy of strong toroidal dipole). The local electric-field (E-field) map for the plasmonic unit cell in the $x y$-plane at the toroidal dipole momentum is shown in Fig. 8.2d, 
demonstrating the strong electric field intensity and localization at the capacitive openings. Formation of a magnetic circular head-to-tail current in the proposed structure is substantiated by plotting the surface current density (j) plane, shown in Fig. 8.2e. In this profile, we plotted the antiparallel current distribution in the proximal resonators, which have significant role in formation of strong toroidal dipole excitation. The perfect antiparallel oscillation of surface currents and magnetic fields are taken place in two sides of a single plasmonic metamolecule gives rise to formation of toroidal spectral feature. Besides, the vectorial profile for the $y z$-plane magnetic-field (H-field) intensity provides complete verification for the creation of closed-loop magnetic field around the central arms of a unit cell (Figure 8.2f).

The effect of the gap distance on the plasmonic response of the toroidal antenna can be further analyzed by comparing the surface current density for three different gap distances. Figure 8.3a illustrates these variations in an $y z$-plane, showing the highest current density for $g=5 \mu \mathrm{m}$ at the toroidal dipole resonant mode, reaching up to $\sim 6.5 \times 10^{5}$ $\mathrm{A} / \mathrm{m}$. This plot implies that stronger surface currents leads to formation of intense shared inductance between neighboring resonators and resulting in substantial confinement of magnetic power in the metamolecules arrays [2,3]. We also quantified the far-field (FF) scattered toroidal power or intensity for varying capacitive gap distances as shown in Figure 8.3b using [3]:

$$
I_{\mathrm{T}}^{F F}=2 \omega^{6}\left|\mathbf{T}_{x y}^{\|}\right|^{2} / 3 c^{5}
$$



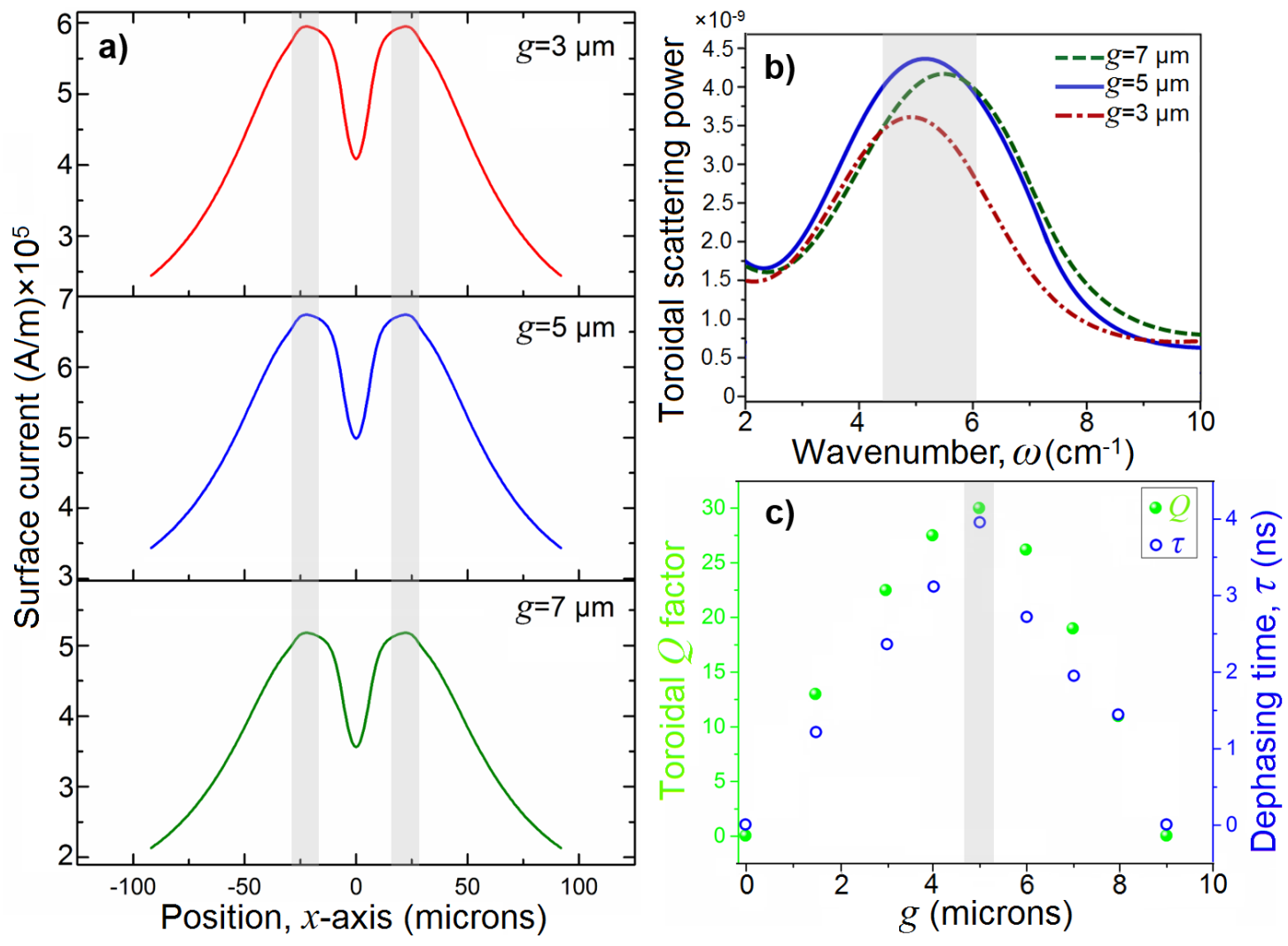

Figure 8.3. Surface current as a function of $x$-axis for three different gap spacing. $b$ ) The toroidal scattering intensity as a function of frequency for three different gap spacing. c) The quality factor of the toroidal lineshape and dephasing time $(\tau)$ as a function of three different gap spacing.

The arisen distinct extremes are correlated with the targeted magnetic toroidal dipole resonant mode with dominant scattering power for the projected toroidal normal to the incident beam direction (z-axis). The electromagnetic computations demonstrate the highest scattered intensity for the gap of $5 \mu \mathrm{m}$. Finally, the quality factor of the induced toroidal momentum in the system for several gap sizes is plotted in Figure 8.3c, using the following equation:

$$
Q=\left(\mathrm{FWHM} / \omega_{\mathrm{T}}\right)-\left(\omega_{\mathrm{T}} / 4(\mathrm{FWHM})^{2}\right)
$$

where FWHM $=\left|\omega_{1}-\omega_{2}\right|$, and $\omega_{\mathrm{T}}$ is the center frequency of toroidal resonance. Here, for the absence of capacitive opening $(g=0 \mu \mathrm{m})$, the toroidal moment does not exist. A 
homogenous increase in the capacitive opening of the curved part of resonators leads to the substantial enhancement in the toroidal quality factor and reaches the highest value $\sim 30$ for $g=5 \mu \mathrm{m}$. Increasing the gap spacing causes drastic reduction in the quality factor due to perturbations in the antiparallel surface currents flowing in proximal resonators, which prevents formation of pronounced toroidal dipole. As a crucial parameter for surface plasmon-enhanced sensing systems [4], this profile also contains the quantified resonance dephasing time $(\tau)$, calculated using Fourier transformation approach. To model the damped harmonic oscillator, we utilized the Cauchy-Lorentz distribution [5], with the dephasing time of toroidal dipole given by: $\tau=2 \hbar / \Delta \omega$, in which $\hbar$ is the reduced Planck's constant. As shown in Fig. 8.3c, the dephasing time of toroidal lineshape increases up to $\sim 3.9 \mathrm{~ns}$ for the resonators with capacitive opening of $5 \mu \mathrm{m}$. The presented optimizations for the proposed plasmonic unit cell should enable stronger interactions with the dielectric biological substances, leading to more precise and accurate sensing via shifting the position of the toroidal lineshape [6].

\subsubsection{The Detection Performance}

Much of the current interest in plasmonic subwavelength on-chip sensors stems from the high sensitivity of asymmetric Fano resonance lineshapes [7-9]. The exquisite sensitivity of Fano-resonant structures to the environmental perturbations led to tailoring of advanced biochemical and biological sensors [10-12]. Despite of the high sensitivity of Fano-resonant nanoscale platforms to the presence of biological nano-objects in direct contact with them, Fano-based $\mathrm{THz}$ plasmonic sensors suffer from low accuracy due to the transparency, low scattering cross-section and non-responsivity of nanoscale bioagents (with the geometry of on the order of $\sim \lambda / 100$ ) and proteins to the incident $\mathrm{THz}$ 


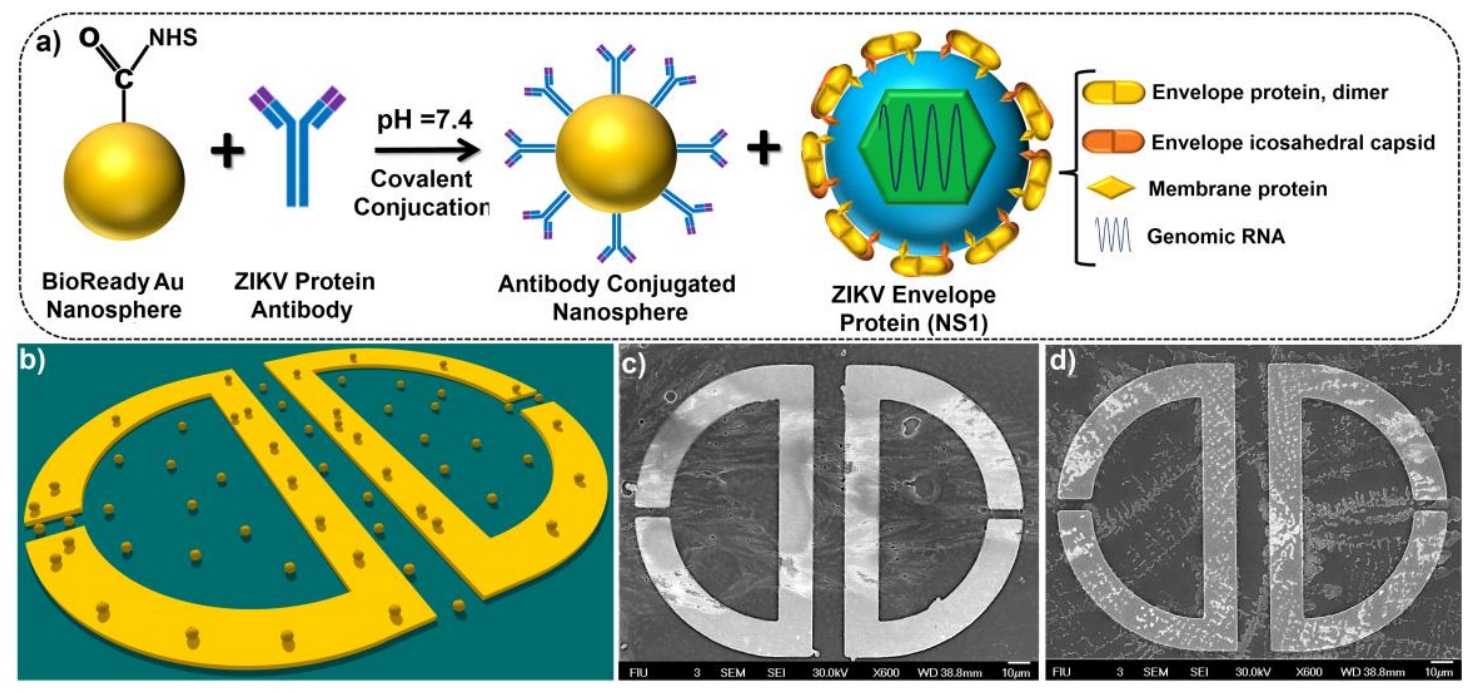

Figure 8.4. a) Schematic flowchart of functionalized gold nanoparticle conjugation with the ZIKV antibody and ZIKV-envelope proteins (NS1) with the explanation for different parts. b) Schematic representation of gold nanoparticles-integrated toroidal unit cells. c) and d) The SEM images of plasmonic metamolecule in the presence of GNPs with antibody and ZIKV envelop proteins, respectively.

wave [13]. On the other hand, THz spectroscopy based biosensing attracts attention due to the advantages like simpler fabrication techniques, low-costs, and high signal to noise ratio [14]. Consequently, $\mathrm{THz}$ plasmonic metasurfaces with the ability to recognize the presence of a specific nanosize biomarkers would be an excellent device for practical biomedical applications. To realize this feature, we integrated the studied plasmonic metasurface and colloidal GNPs with each other to enhance the sensitivity of the metasurface. Using functionalized and conjugated colloidal GNPs, we investigated the change in the toroidal response to detect the presence of a specific biomarker.

To this end, surface functionalized gold colloids (with the diameter of $42 \mathrm{~nm}$, and with an OD of 20.09, BioReady NHS dried, NanoComposix) were used with robust covalent conjugation to primary amines $\left(-\mathrm{NH}_{2}\right)$ of proteins. For the conjugation of ZIKV antibody with the GNPs, a reconstitution buffer was prepared by combining $1 \mathrm{~mL}$ of reaction buffer was with $35 \mu \mathrm{L}$ of purified ZIKV antibody. Then the prepared buffer was 


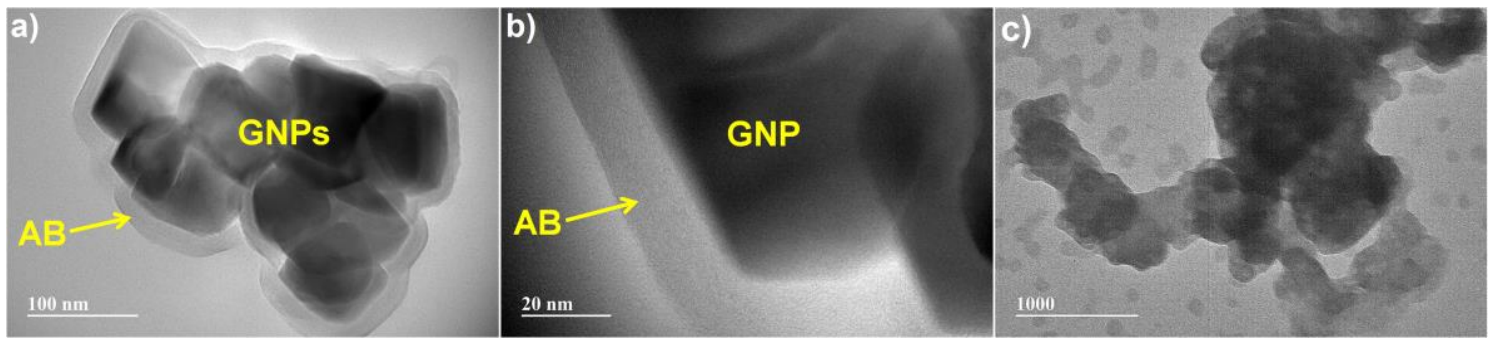

Figure 8.5. a), (b) TEM images of functionalized GNPs binding with antibody in two different scales. (c) The TEM image of ZIKV envelope proteins captured by antibody-conjugated GNPs. In this picture, the $\mathrm{AB}$ stands for antibody.

added to the NHS dried gold colloid, sonicated for 30 seconds. The solution is incubated while rotating at room temperature for 60 minutes. Next, we added $10 \mu \mathrm{L}$ of quencher to deactivate the possible remaining active NHS-esters. The solution centrifuged at 3600 $\mathrm{RCF}$ for $10 \mathrm{~min}$, then the supernatant removed cautiously and resuspended with $1 \mathrm{~mL}$ of reaction buffer including a sonication until fully resuspension. By repeating the previous protocol from centrifuging process with the same protocol, finally, we added $50 \mu \mathrm{L}$ of conjugate diluent by sonication. The conjugated samples were stored at $4{ }^{\circ} \mathrm{C}$. To conjugate the prepared GNPs with the fabricated toroidal unit cells, we dropped $10 \mu \mathrm{L}$ (1 $\mathrm{mg} / \mathrm{mL}$ ) of colloidal solution to the chips and wait till dried. Then, the prepared targeted ZIKV biomarker proteins were added to the prepared plasmonic metamolecules and stored at $4{ }^{\circ} \mathrm{C}$.

Similar to the previous chapter, we used ZIKV-envelope proteins (NS1) with the molecular-weight of $\approx 13 \mathrm{kDa}$ as a biological target in the presence of respective ZIKV antibody, as shown schematically in Figure 8.4a with the subsequent components of ZIKV protein and illustration of ZIKV antibody and ZIKV envelope proteins conjugation. The GNPs here are used to trap the proteins (see the schematic picture and SEM image in Figure 8.4b and 8.4c, respectively) and change the refractive index of the 
medium to facilitate substantial shift in the position of toroidal dipole. For simplicity, we used fixed concentration of nanoparticles $(10 \mu \mathrm{g} / \mathrm{mL}$ or $\sim 77$ picomolar $(\mathrm{pM}))$, while the injected envelope protein concentration was varied between $1 \mathrm{fg} / \mathrm{mL}$ and $1 \mu \mathrm{g} / \mathrm{mL}$. Figure 8.4d illustrates the SEM image of the toroidal unit cell with the presence of ZIKV envelope proteins, captured by functionalized nanoparticles. To show the binding quality of ZIKV antibody and ZIKV envelope proteins to the GNPs, we illustrated the corresponding transmission electron microscopy (TEM) pictures in Figure 8.5a-8.5c to show the uniform, and strong binding of the bio-molecules to the nanoparticles, which enhances the sensitivity of the metasensor. The corresponding TEM pictures were taken by PHILIPS CM200 machine.

The experimentally obtained $\mathrm{THz}$ transmission spectra with distinct toroidal responses for different concentrations of ZIKV envelope proteins are presented in Figure 8.6a. For the absence of colloidal GNPs and biological agents and in the ambient atmosphere, the magnetic toroidal moment appears at $6.67 \mathrm{~cm}^{-1}$. By introducing the ZIKV-antibody-conjugated GNPs to the metasurface chips, the toroidal mode red-shifts to $6.53 \mathrm{~cm}^{-1}$, while for the absence of nanoparticles and direct binding of ZIKV antibody to the metamolecule, the resonant mode slightly shifts to $6.6 \mathrm{~cm}^{-1}$. Adding ZIKV envelope proteins with increasing concentration from $1 \mathrm{fg} / \mathrm{mL}$ to $100 \mathrm{pg} / \mathrm{mL}$ did not cause any shift in the toroidal moment and it stayed at the initial position of the presence of ZIKV antibody in both cases. The major toroidal shift in the position of toroidal resonance was observed for $1 \mathrm{ng} / \mathrm{mL}$ concentration of biomarker proteins for the presence of GNPs-conjugated system (red-shifts to $6.33 \mathrm{~cm}^{-1}$ ), while the toroidal lineshape did not shift for the bare metamolecule with the presence of the same amount of envelope 

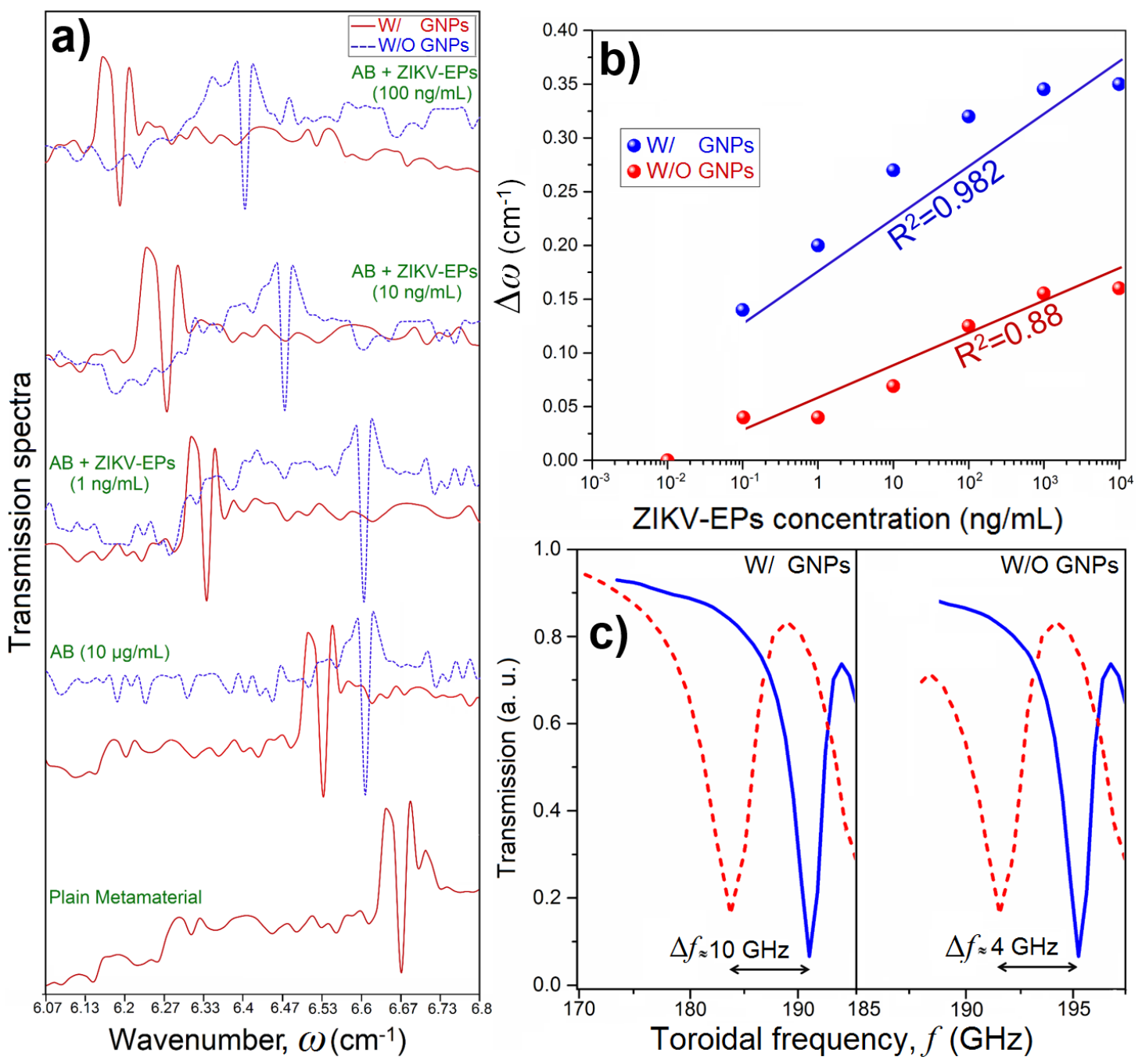

Figure 8.6. a) The transmission amplitude spectra for the fabricated metasurfaces in both $\mathrm{W} /$ and W/O GNPs regimes in the presence of ZIKV antibody and ZIKV envelope proteins with different concentrations. b) The toroidal resonance shift as a function of ZIKV envelope proteins concentration W/ and W/O GNPs with the corresponding determination coefficient. c) The magnified transmission spectra as a function of frequency, showing the maximum shift of the toroidal moment in the presence and absence of GNPs attached to the system.

proteins. Further increase in the concentration of biomarker proteins leads to further shift in the position of toroidal dipole, while for the presence of $100 \mathrm{ng} / \mathrm{mL}$ of proteins, the toroidal mode appears at $6.13 \mathrm{~cm}^{-1}$. On the other hand, the bare unit cell starts to show reaction to the presence of ZIKV envelope proteins at the concentration of $10 \mathrm{ng} / \mathrm{mL}$ (red-shifts to $6.47 \mathrm{~cm}^{-1}$ ). Moreover, for the concentration of $100 \mathrm{ng} / \mathrm{mL}$ of proteins, the 

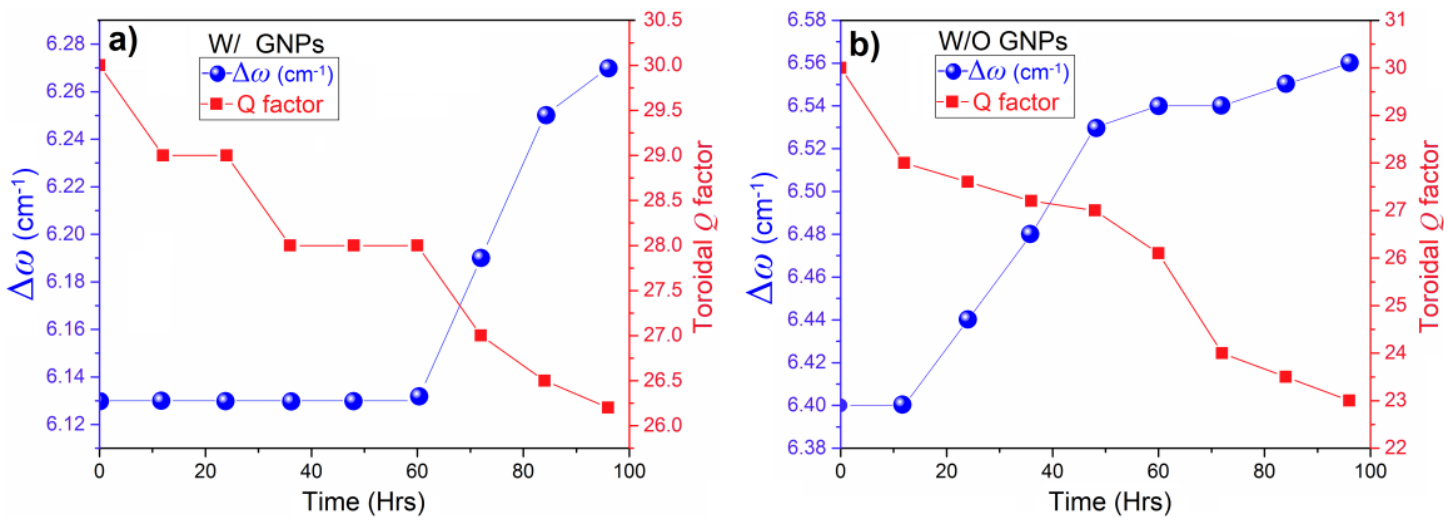

Figure 8.7. a) and (b) The toroidal resonance shift $(\Delta \omega)$ and quality factor as a function to time in hours for the presence and absence of GNPs, respectively.

toroidal dipole red-shifts slightly to $6.4 \mathrm{~cm}^{-1}$. It should be underlined that the position of toroidal moment shows trivial shift for the concentrations of proteins more than $\geq 1$ $\mu \mathrm{g} / \mathrm{mL}$. The toroidal dipole shifts $(\Delta \omega)$ as a function of concentration is plotted in a semilogarithmic graph in Figure 8.6b for the presence and absence of GNPs attached to the system. This profile clearly shows how the presence of GNPs improved the sensitivity of the toroidal metasurface significantly. A large red-shift was obtained for GNPsintroduced system around $\Delta \omega \sim 0.35 \mathrm{~cm}^{-1}(\Delta f \sim 10 \mathrm{GHz}$, Figure 8.6c) for the biomarker concentration of $100 \mathrm{ng} / \mathrm{mL}(\sim 77 \mathrm{pM})$ with the coefficient of determination $R^{2}=0.982$. For the system without plasmonic nanoparticles, the largest shift was around $\Delta \omega \sim 0.14$ $\mathrm{cm}^{-1}\left(\Delta f \sim 4 \mathrm{GHz}\right.$, Figure 7.6c), with determination coefficient of $R^{2}=0.88$.

In continue, we studied the repeatability and stability of the $\mathrm{THz}$ toroidal response by time as demonstrated in Figures $8.7 \mathrm{a}$ and $8.7 \mathrm{~b}$ for nanoparticle integrated and bare metasurfaces, respectively. These experiments were done at the presence of $100 \mathrm{ng} / \mathrm{mL}$ of ZIKV envelope proteins bound to the system. We compared the toroidal shifts and the corresponding quality factors as a function of time. For the GNPs-integrated metasurface, 
the resonance shift remains fixed for approximately 60 hours, while a gradual blue-shift in the position of is monitored afterwards. However, the blue-shift and deterioration of the toroidal mode are drastic in bare metamolecules in the absence of GNPs (Figures 8.7b). The same behavior observed for the quality factor, where the quality factor of the bare unit cells monotonically declined. This deterioration also included a significant blueshift in the position of magnetic resonant mode to the higher energies. As a final comparison between two types of studied samples, we accurately quantified the limit of detection (LoD) and sensitivity of the metasensors as: $\sim 560 \mathrm{pg} / \mathrm{mL}$ and 5.81 $\mathrm{GHz} / \log (\mathrm{pg} / \mathrm{mL})$ for the GNP-integrated metasurface and $12 \mathrm{ng} / \mathrm{mL}$ and 2.25 $\mathrm{GHz} / \log (\mathrm{pg} / \mathrm{mL})$ for the bare metasurface. Recently, $\mathrm{THz}$ based biosensors with noteworthy sensitivities for detection of bio-molecules with larger molecular weights such as Avidin ( 68 kDa) [15] and Rat IgG ( 150 kDa) [16] have been reported. Demonstration of high-sensitivity detection of ZIKV envelope proteins with much lower molecular weight $(\sim 13 \mathrm{kDa})$ shows the superiority of the proposed toroidal plasmonic $\mathrm{THz}$ metasensor over the previously reported $\mathrm{THz}$ based biosensors. Use of plasmonic GNPs enabled strong binding of proteins and further improved the sensitivity and responsivity.

\subsubsection{Conclusions}

To conclude, we have analyzed the plasmonic response of a multipixel metamolecule with the ability to support high quality factor toroidal dipole moment along the $\mathrm{THz}$ domain. By introducing gold nanospheres to the system, we have shown that the toroidal resonance can be tuned effectively. To show the exotic dependency of the induced resonant mode to the surrounding variations, by using infectious proteins to the system, 
we investigated the resonant mode behavior for different concentrations of biomarker proteins. To show the influence of the presence of plasmonic GNPs, we provided the experimental data for the presence and absence of gold particles. The results verified that in the presence of gold nanoparticles the sensitivity of the metasensor can be enhanced and large resonance shift can be achieved. In addition, due to strong binding of nanoparticles and biomarker nano-objects, a significant repeatability was observed for the chip integrated with GNPs for more than $75 \mathrm{~h}$. We believe that this study paves new methods to provide highly sensitive, efficient, and promising $\mathrm{THz}$ metamaterials for detection of biomarker agents.

\subsection{References}

1. Ordal, M. A., Bell, R. J., Alexander, R. W., Long, L. L., \& Querry, M. R. (1987). Optical properties of $\mathrm{Au}, \mathrm{Ni}$, and $\mathrm{Pb}$ at submillimeter wavelengths. Applied Optics, 26(4), 744-752.

2. Cojocari, M. V., Schegoleva, K. I., \& Basharin, A. A. (2017). Blue-shift and phase tunability in planar $\mathrm{THz}$ metamaterials: the role of losses and toroidal dipole contribution. Optics Letters, 42(9), 1700-1703.

3. Li, J., Zhang, Y., Jin, R., Wang, Q., Chen, Q., \& Dong, Z. (2014). Excitation of plasmon toroidal mode at optical frequencies by angle-resolved reflection. Optics Letters, 39(23), 6683-6686.

4. Liu, N., Tang, M. L., Hentschel, M., Giessen, H., \& Alivisatos, A. P. (2011). Nanoantenna-enhanced gas sensing in a single tailored nanofocus. Nature materials, 10(8), 631-636.

5. Miyamaru, F., Tanaka, M., \& Hangyo, M. (2006). Effect of hole diameter on terahertz surface-wave excitation in metal-hole arrays. Physical Review B, 74(15), 153416.

6. Yahiaoui, R., Strikwerda, A. C., \& Jepsen, P. U. (2016). Terahertz plasmonic structure with enhanced sensing capabilities. IEEE Sensors Journal, 16(8), 24842488. 
7. Verellen, N., Sonnefraud, Y., Sobhani, H., Hao, F., Moshchalkov, V. V., Dorpe, P. V., Nordlander, P., \& Maier, S. A. (2009). Fano resonances in individual coherent plasmonic nanocavities. Nano Letters, 9(4), 1663-1667.

8. Hao, F., Sonnefraud, Y., Dorpe, P. V., Maier, S. A., Halas, N. J., \& Nordlander, P. (2008). Symmetry breaking in plasmonic nanocavities: subradiant LSPR sensing and a tunable Fano resonance. Nano letters, 8(11), 3983-3988.

9. Ahmadivand, A., Karabiyik, M., \& Pala, N. (2015). Inducing multiple Fano resonant modes in split concentric nanoring resonator dimers for ultraprecise sensing. Journal of Optics, 17(8), 085104.

10. Zeng, B., Gao, Y., \& Bartoli, F. J. (2014). Rapid and highly sensitive detection using Fano resonances in ultrathin plasmonic nanogratings. Applied Physics Letters, 105(16), 161106.

11. Erwin, W. R., \& Bardhan, R. (2016). Directional Scattering and Sensing with Bimetallic Fanocubes: A Complex Fano-Resonant Plasmonic Nanostructure. Phys. Chem. C, 120(51), 29423-29431.

12. Lovera, A., Gallinet, B., Nordlander, P., \& Martin, O. J. (2013). Mechanisms of Fano resonances in coupled plasmonic systems. ACS Nano, 7(5), 4527-4536.

13. Xie, L., Gao, W., Shu, J., Ying, Y., \& Kono, J. (2015). Extraordinary sensitivity enhancement by metasurfaces in terahertz detection of antibiotics. Scientific Reports, 5,8671 .

14. Park, S. J., Hong, J. T., Choi, S. J., Kim, H. S., Park, W. K., Han, S. T., Park, J. Y., Lee, S., Kim, D. S., \& Ahn, Y. H. (2014). Detection of microorganisms using terahertz metamaterials. Scientific Reports, 4, 4988.

15. Ahmadivand, A., Gerislioglu, B., Tomitaka, A., Manickam, P., Kaushik, A., Bhansali, S., Nair, M., \& Pala, N. (2018). Rapid Detection of Infectious Envelope Proteins by Magnetoplasmonic Toroidal Metasensors. Biomedical Optics Express, 9(2), 373-368.

16. Yan, S., Xia, L., Wei, D., Cui, H. L., \& Du, C. (2016). Terahertz biosensing of protein based on a metamaterial. IEEE Manipulation, Manufacturing and Measurement on the Nanoscale, 3M-NANO, 327-330. 


\section{CHAPTER 9}

\section{Conclusions}

In this dissertation, we have presented our work on the plasmonic response of several metallic and metallodielectric nano- and microstructures to support conventional and novel resonant moments along a wide range of spectrum. Initially, by focusing on the classical electromagnetic response of plasmonic subwavelength objects, we analyzed the theoretical mechanism behind the formation of EM modes along the UV to the THz band of spectrum. Then, the plasmonic response of strongly coupled and hybridized systems were analyzed numerically and theoretically by providing the description for the formation of dark plasmons and generation of Fano spectral feature. Using this advantage of significant absorption cross-section at the Fano lineshape wavelength, we studied our developed plasmonic devices such as precise biochemical sensors and photodetectors. The study of plasmonic moments were continued by providing an explanation for the excitation of novel quantum-mechanical-based plasmonic moments in nanoparticles with atomic scale offset openings and also in directly connected nanoparticles with a conductive link. We have shown that in both cases, the optically excited charges tunnels (in atomic gap regime) and shuttles (in directly connected regime) and leads to the formation of CTP resonances. By carrying out highly accurate numerical analysis, we enhanced the tunability of the induced CTP moment by employing optothermally controllable substances in the geometry of the plasmonic nanostructures. Such a strategy

led us to develop nanoplasmonic metaswitches to operate at the telecommunication band with high speed and low insertion losses. 
On the other hand, we showed that subwavelength multipixel plasmonic metamolecules and meta-atoms can be tailored to support a novel and third family of multipoles, known as toroidal moments. Our comprehensive investigations for the excitation of toroidal moments including theoretical physics, numerical studies, and experimental results were described along the work. In addition, we have developed $\mathrm{THz}$ plasmonic metasurafces for practical switching and modulation applications based on toroidal dipolar resonances.

Ultimately, by explaining the applications of plasmonic platforms in advanced medical, pharmacology and clinical applications, we tailored plasmonic $\mathrm{THz}$ metamaterials for biosensing applications. To this end, we developed artificial plasmonic metamolecules to sustain strong toroidal resonant modes across the $\mathrm{THz}$ band. Both numerical and experimental studies were employed with high accuracy to effectively predict and measure the toroidal response of different types of planar plasmonic metamaterials. We showed that the exquisite features of $\mathrm{THz}$ waves allows for developing non-contact, nonpoisonous, non-destructive, and cost-effective sensing devices for identification of low molecular-weight molecules in the range of a few kDa. To this end, we designed and fabricated planar toroidal metamolecules capable to support high quality narrow toroidal lineshapes across the sub- $\mathrm{THz}$ spectrum. Then, the developed platforms were employed for the detection of a wide spreading infectious envelope protein for Zika virus. Our analyses have shown that in the presence of biomarker envelope proteins and its respective antibody, the toroidal resonance significantly red-shifts, leading to obtaining high sensitivity to the environmental variations. 
We further enhanced the sensitivity and repeatability of plasmonic metasensors by carrying out a novel approach. To this end, we introduced plasmonic nanoscale particles to the microsize artificial metamolecules to enhance the binding of biomarker proteins and increase the sensitivity of the biodetection system to the presence of nano-objects around the structures. The experimental studies for Zika-virus envelope proteins showed that the metasurface reflects significant sensitivity and large toroidal dipole moment shift to the presence of bio-agents. This protocol allowed us to optimize the repeatability of the metasensor from three days in the classical system to five days in the nanoparticlesintegrated system. We envision that our developed plasmonic metasensors are potential candidates for practical quick infection diagnosis, cost-effective and real-time pharmacology applications owing to the non-destructive and harmless interaction with biological tissues in both in vivo and in vitro assays.

\subsection{Key Contributions of the Dissertation}

In this dissertation, we reported on the substantial achievements that have been done for the first time. Here, we analyzed the possibility of the excitation of various types of classical and novel electromagnetic moments using plasmonic subwavelength structures such as Fano resonances, CTP, and toroidal moments. Employing optothermally and optoelectronically controllable substances for designing our nanostructures, as a very first report, we efficiently and successfully tuned the plasmonic resonant moments along the targeted spectra and developed functional plasmonic nanodevices such as modulators, sensors, switches, and photodetectors. 
By tailoring and fabricating artificial $\mathrm{THz}$ plasmonic metamolecules, we developed toroidal metamaterials with high sensitivity to the presence of nanoscale biological objects and biomarkers proteins. By choosing ZIKV envelope proteins as target biomarkers, for the first time, we detected biomarker agents with the size of around 40 $\mathrm{nm}$ and molecular weight of $\sim 13 \mathrm{kDa}$ in the presence of its respective antibody. We also, enhanced the sensitivity, selectivity, and repeatability of the toroidal metasurfaces by introducing GNPs to the microscale system. This method and having high resolution spectral feature allowed us to enhance the binding of biological objects to the metasurface and increase the sensitivity and toroidal resonance shift due to the variations in the concentration of the substance around the medium. The non-invasive and nonpoisonous feature of $\mathrm{THz}$ waves and employing the toroidal plasmonic technology, we provided label-free, real-time, room-temperature, fast, cost-effective, promising, and accurate plasmonic metasensors as leading structures for advanced biosensing purposes.

\subsection{Recommendations for the Future Work}

The THz plasmonic metamaterials technology can be also enhanced for the detection of various biomarker and infectious agents such as (Alzheimer's disease, etc.) with high accuracy and reliability. High resolution and ultra-narrow spectra lineshapes can be induced by well-engineered planar and 3D artificial plasmonic metamolecules to provide enhanced sensing. Moreover, excitation of tunable resonant moments in a given metamaterial leads to having multispectral platform to operate at different frequencies depending on the application and targeted spectral range. We envision that this work paves new methods for developing advanced, next-generation nanophotonic devices as well as nanomedical applications based on plasmonic technology. 
VITA

ARASH AHMADIVAND

2003-2007

B.Sc. Electrical and Electronic Engineering, Islamic Azad

University, Birjand, Iran.

2009-2011

M.Sc.Electrical and Electronic Engineering, Islamic Azad

University, Ahar, Iran.

2014-2018

Doctoral Candidate (PhD), Florida International University,

Miami, Florida, USA

\section{PUBLICATIONS}

1. Ahmadivand, A., Gerislioglu, B., Tomitaka, A., Manickam, P., Kaushik, A., Bhansali, S., Nair, M., \& Pala, N. (2018). Rapid Detection of Infectious Envelope Proteins by Magnetoplasmonic Toroidal Metasensors. Biomedical Optics Express, 9(2), 373-368.

2. Ahmadivand, A., Gerislioglu, B., \& Pala, N. (2017). Active Control over the Interplay between the Dark and Hidden Sides of Plasmonics Using Metallodielectric Au$\mathrm{Ge}_{2} \mathrm{Sb}_{2} \mathrm{Te}_{5}$ Unit Cells. The Journal of Physical Chemistry C, 121(36), 19966-19974.

3. Ahmadivand, A., Gerislioglu, B., Manickam, P., Kaushik, A., Bhansali, S., Nair, M., \& Pala, N. (2017). Rapid Detection of Infectious Envelope Proteins by Magnetoplasmonic Toroidal Metasensors. ACS Sensors, 2(9), 1359-1368.

4. Ahmadivand, A., Gerislioglu, B., \& Pala, N. (2017). Graphene optical switch based on charge transfer plasmons. Physica Status Solidi-Rapid Research Letters, 11(11), 1700285 .

5. Ahmadivand, A., Gerislioglu, B., Sinha, R., Karabiyik, M., \& Pala, N. (2017). Optical Switching Using Transition from Dipolar to Charge Transfer Plasmon Modes in $\mathrm{Ge}_{2} \mathrm{Sb}_{2} \mathrm{Te}_{5}$ Bridged Metallodielectric Dimers. Scientific Reports, 7, 42807.

6. A. Ahmadivand, Gerislioglu, B., \& Pala, N. (2017). Large-modulation-depth polarization-sensitive plasmonic toroidal terahertz metamaterial, IEEE Photonics Technology Letters, 29(21), 1860-1863. 
7. Ahmadivand, A., Gerislioglu, B., \& Pala, N. (2017). Azimuthally and radially excited charge transfer plasmon and Fano lineshapes in conductive sublayer-mediated nanoassemblies. J. Opt. Soc. Am. A, 34(11), 2052-2056.

8. Gerislioglu, B., Ahmadivand, A., \& Pala, N. (2017). Functional quadrumer clusters for switching between Fano and charge transfer plasmons. IEEE Photonics Technology Letters, 29(24), 2226-2229.

9. Vabbina, P. K., Sinha, R., Ahmadivand, A., Karabiyik, M., Gerislioglu, B., Awadallah, O., \& Pala, N. (2017). Sonochemical Synthesis of Zinc Oxide Core-Shell Nanorod Radial PN Homojunction Ultraviolet Photodetector. ACS Applied Materials \& Interfaces, 9(23), 19791-19799.

10. Gerislioglu, B., Ahmadivand, A., Karabiyik, M., Sinha, R., \& Pala, N. (2017). VO $2_{2}^{-}$ Based Reconfigurable Antenna Platform with Addressable Microheater Matrix. Advanced Electronic Materials, 3(9), 1700170.

11. Ahmadivand, A., Gerislioglu, B., Sinha, R., Vabbina, P. K., Karabiyik, M., \& Pala, N. (2017). Excitation of Terahertz Charge Transfer Plasmons in Metallic Fractal Structures. Journal of Infrared, Millimeter, and Terahertz Waves, 38(8), 992-1003.

12. Ahmadivand, A., Sinha, R., Karabiyik, M., Vabbina, P. K., Gerislioglu, B., Kaya, S., \& Pala, N. (2017). Tunable THz wave absorption by graphene-assisted plasmonic metasurfaces based on metallic split ring resonators. Journal of Nanoparticle Research, 19(1), 3.

13. Ahmadivand, A., Sinha, R., \& Pala, N. (2017). Magnetic Fano resonances in alldielectric nanocomplexes under cylindrical vector beams excitation. Optics \& Laser Technology, 90, 65-70.

14. Ahmadivand, A., Sinha, R., Vabbina, P. K., Karabiyik, M., Kaya, S., \& Pala, N. (2016). Hot electron generation by aluminum oligomers in plasmonic ultraviolet photodetectors. Optics Express, 24(12), 13665-13678.

15. Ahmadivand, A., Sinha, R., Gerislioglu, B., Karabiyik, M., Pala, N., \& Shur, M. (2016). Transition from capacitive coupling to direct charge transfer in asymmetric terahertz plasmonic assemblies. Optics Letters, 41(22), 5333-5336.

16. Ahmadivand, A., Sinha, R., \& Pala, N. (2016). Resonance coupling in plasmonic nanomatryoshka homo-and heterodimers. AIP Advances, 6(6), 065102.

17. Ahmadivand, A., Golmohammadi, S., Karabiyik, M., \& Pala, N. (2015). Fano resonances in complex plasmonic necklaces composed of gold nanodisks clusters for enhanced LSPR sensing. IEEE Sensors Journal, 15(3), 1588-1594.

18. Ahmadivand, A., Karabiyik, M., \& Pala, N. (2015). Photodetectors: Materials, devices and applications in communications and imaging technologies (Ch. 6). Woodhead Publishing, Elsevier. 